Dimitar Georgiev Vlaykov

SUB-GRID SCALE MODELLING OF

COMPRESSIBLE MAGNETOHYDRODYNAMIC TURBULENCE:

DERIVATION AND A PRIORI ANALYSIS

Dissertation 



\title{
Sub-grid scale modelling of compressible magnetohydrodynamic turbulence: derivation and a priori analysis
}

\author{
Dissertation \\ for the award of the degree \\ "Doctor of Philosophy" (Ph.D.) Division of Mathematics and \\ Natural Sciences \\ of the Georg-August-Universität Göttingen \\ within the doctoral program PROPHYS \\ of the Georg-August University School of Science (GAUSS) \\ submitted by \\ Dimitar Georgiev Vlaykov
}

from Pazardzhik, Bulgaria

Göttingen, 2015 


\section{$\underline{\text { Thesis committee }}$}

PD Dr. Wolfram Schmidt, Hamburger Sternwarte, Universität Hamburg Prof. Dr. Jörg Büchner, Max-Planck-Institut für Sonnensystemforschung Prof. Dr. Jens Niemeyer, Institut für Astrophysik, Universität Göttingen

\section{$\underline{\text { Referees }}$}

Referent: PD Dr. Wolfram Schmidt, Hamburger Sternwarte, Universität Hamburg Korreferent: Prof. Dr. Jens Niemeyer, Institut für Astrophysik, Universität Göttingen

\section{Additional members of the examination board}

Prof. Dr. Jörg Büchner, Max-Planck-Institut für Sonnensystemforschung

Prof. Dr. Gert Lube, Institut für Numerische und Angewandte Mathematik, Universität Göttingen

Prof. Dr. Ansgar Reiners, Institut für Astrophysik, Universität Göttingen

Prof. Dr. Andreas Tilgner, Institut für Geophysik, Universität Göttingen

Date of the oral examination: 


\section{Motivation and Purpose}

Turbulence is one of the oldest and trickiest unsolved problems in our understanding of the universe. The addition of magnetic fields increases its complexity exorbitantly. There are numerous astrophysical examples where the turbulent dynamics of magnetised plasma is critical, from the largest to the smallest scales: it may hold the key to explaining the amplification of the magnification fields on galactic and super-galactic scales through small-scale dynamos, the coronal heating through turbulent reconnection, the self-regulation of star formation rate through turbulent support, et cetera. Due to the large degree of non-linearity of turbulence, numerical simulations are a preferred tool to address many of these problems. In the astrophysical context, direct numerical simulations of compressible magnetohydrodynamic turbulence, the simplest theory which contains all the basic physical ingredients, are often computationally intractable due to the prohibitively large dynamical range between the dissipative and integral scales. The finite numerical resources impose a grid with finite resolution below which no self-consistent information is computable. In order to incorporate the effects of the sub-grid scale dynamics, one needs to provide a physically justified model.

The purpose of this thesis is to develop a self-consistent model which is faithful to the sub-grid scale dynamics of compressible magnetohydrodynamic turbulence. This is performed by means of a priori analysis of pre-existing data. The data has sufficient resolution for a well delineated power-law scaling, i.e. turbulent, range in the energy spectrum. An attempt is made to identify and investigate critical properties of the subgrid scale dynamics in the simulations of developed statistically homogeneous, isotropic and stationary compressible magnetohydrodynamic turbulence. The proposed model is developed based on a deconvolution approach. It is validated a priori against the data and alternative models currently in circulation based on a set of dynamical and geometrical, frame-independent diagnostic fields. 



\section{Contents}

1 Introduction 3

1.1 Fluid dynamics and MHD . . . . . . . . . . . . . . 3

1.1.1 Magnetohydrodynamic equations ............. . . 4

1.1.2 Scale-separation: RANS, ILES, LES . . . . . . . . . . . 5

1.2 Introduction to LES formalism . . . . . . . . . . . . . . . . 7

1.2.1 Filtering operator . . . . . . . . . . . . . 7

1.2.2 Filter selection and choice . . . . . . . . . . . . . . . 9

1.3 Magnetohydrodynamic equations for LES . . . . . . . . . . . . . . . . . 10

1.4 Validation methods . . . . . . . . . . . . . . . . . . . 14

1.5 Introduction of sample data . . . . . . . . . . . . . . . . . 15

1.5.1 Analysed parameter space . . . . . . . . . . . . . 15

1.5.2 Choice of filter scale . . . . . . . . . . . . . . 19

2 Sub-grid scale models $\quad 21$

2.1 Non-linear model . . . . . . . . . . . . . . . . . . . . . . . . . . . . 22

2.1.1 Yeo-Bedford series expansion ............... 22

2.1.2 Compressible extension via recurrence relation . . . . . . . . . . 24

2.1.3 Link with ancestor and sibling models . . . . . . . . . . . . 26

2.1.4 Model renormalization/regularization . . . . . . . . . . . . 26

2.2 Alternative models . . . . . . . . . . . . . . . . . . . . . 28

2.2.1 Scale-similarity model . . . . . . . . . . . . . . . 28

2.2.2 Eddy-dissipation and dynamo models . . . . . . . . . . . . 30

2.3 Sub-grid scale energy and cross-helicity models . . . . . . . . . . . . . 34

2.3.1 Implicit models . . . . . . . . . . . . . . . . . 35

2.3.2 Evolution equations and Smagorinsky approximation . . . . . . 36

2.3.3 Realizability condition ... . . . . . . . . . . . . 38

2.3.4 Dissipation/backscatter properties of selected models . . . . . . 39

2.4 Final remarks . . . . . . . . . . . . . . . . . . . . . . . 41

3 Analysis of the compressible MHD LES equations and hypothesis testing 45

3.1 Analysis of the primary equations . . . . . . . . . . . . 45

3.2 Induction equation and the EMF . . . . . . . . . . . . . . . . 47

3.3 Momentum equation and the SGS stress . . . . . . . . . . . . 50

3.3.1 Tensor analysis of the SGS stress . . . . . . . . . . . 53

3.3.2 SGS pressure . . . . . . . . . . . . . . . 60

3.4 Energy equations . . . . . . . . . . . . . . . . 63

3.4.1 Term comparison . . . . . . . . . . . . 66 
3.5 Cross-helicity equation . . . . . . . . . . . . . . 70

4 Model Validation $\quad 75$

4.1 Sub-grid scale energy models . . . . . . . . . . . . . . . . . . . . 77

4.2 Deviatoric SGS kinetic stress: $\tau^{\mathrm{u}} \ldots \ldots \ldots \ldots$

4.3 Deviatoric magnetic SGS stress: $\tau^{\mathrm{b} *} \ldots \ldots \ldots \ldots$

4.4 Deviatoric total SGS stress: $\tau^{*} \ldots \ldots \ldots \ldots$. . . . . . . . . . . . . . . . . . . . 94

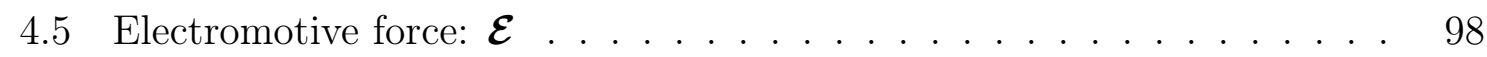

4.6 Summary . . . . . . . . . . . . . . . . . . . . . 102

5 Conclusions and Outlook 107 


\section{Notation}

,- differentiation operator, e.g. $f_{, i}=\partial_{i} f=\frac{\partial f}{\partial x_{i}}$

$\star-$ convolution operator

$\frac{\mathrm{D}}{\mathrm{D} t}=\frac{\partial}{\partial t}+\mathbf{u} \cdot \nabla-$ Lagrangian derivative

$\square$ - placeholder for generic expression

$\widehat{\square}$ - closure expression for $\square$

$\square$ - filtered version of

$\widetilde{\square}$ - mass-weighted (Favre) filtered version of $\square$, equivalent to $\frac{\overline{\rho \square}}{\bar{\rho}}$

$\square^{*}$ - deviatoric (traceless) part of $\square$, equivalent to $\square-\frac{1}{3} \operatorname{Tr}(\square) \mathrm{I}$ (only for $2^{\text {nd }}$ rank tensors)

$\rho$ - mass density

$\mathbf{u}$ - velocity field

B - magnetic field

$P$ - thermal pressure

$\Delta$ - filter scale (in physical space)

$\mathcal{S}_{i j} \equiv \frac{1}{2}\left(\partial_{i} u_{j}+\partial_{j} u_{i}\right)$ - kinetic rate-of-strain

$\mathcal{M}_{i j} \equiv \frac{1}{2}\left(\partial_{i} B_{j}+\partial_{j} B_{i}\right)$ - magnetic rate-of-strain

$\Omega_{i j}=\frac{1}{2}\left(\partial_{i} u_{j}-\partial_{j} u_{i}\right)-$ vorticity tensor

$J_{i j}=\frac{1}{2}\left(\partial_{i} B_{j}-\partial_{j} B_{i}\right)-$ current tensor

$\Omega \equiv \nabla \times \mathbf{u}-$ vorticity vector

$\omega \equiv \sqrt{\Omega^{2}}-$ enstrophy

$\mathbf{J} \equiv \nabla \times \mathbf{B}-$ current vector

$\delta$ - Kronecker delta (symmetric isotropic tensor)

$\epsilon$ - Levi-Civita symbol (anti-symmetric isotropic pseudo-tensor, 3 dimensions)

$G$ - filter kernel

I - the identity (tensor)

$\tau^{\mathrm{u}}$ - kinetic SGS stress tensor

$\tau^{\mathrm{b}}$ - magnetic SGS stress tensor

$\tau \equiv \tau^{\mathrm{u}}-\tau^{\mathrm{b}}-$ total SGS stress tensor

$\bar{E}^{\square} \equiv G \star\left(E^{\square}\right)$ - total filtered $\square$ energy

$\bar{E}^{\mathrm{u}} \equiv G \star\left(\frac{1}{2} \rho \mathbf{u}^{2}\right)-$ total filtered kinetic energy

$\bar{E}^{\mathrm{b}} \equiv G \star\left(\frac{1}{2} \mathbf{B}^{2}\right)-$ total filtered magnetic energy

$E_{\mathrm{res}}^{\mathrm{u}} \equiv \frac{1}{2} \bar{\rho} \tilde{\mathbf{u}}^{2}-$ resolved kinetic energy

$E_{\text {res }}^{\mathrm{b}} \equiv \frac{1}{2} \overline{\mathbf{B}}^{2}$ - resolved magnetic energy 
$E_{\mathrm{sgs}}^{\mathrm{u}} \equiv \bar{E}^{\mathrm{u}}-E_{\mathrm{res}}^{\mathrm{u}}=\frac{1}{2} \operatorname{Tr}\left(\tau^{\mathrm{u}}\right)-$ kinetic SGS energy

$E_{\mathrm{sgs}}^{\mathrm{b}} \equiv \bar{E}^{\mathrm{b}}-E_{\mathrm{res}}^{\mathrm{b}}=\frac{1}{2} \operatorname{Tr}\left(\tau^{\mathrm{b}}\right)-$ magnetic SGS energy

$\mathcal{E}_{i j}=\left(\overline{u_{i} B_{j}-u_{j} B_{i}}\right)-\left(\tilde{u}_{i} \bar{B}_{j}-\tilde{u}_{j} \bar{B}_{i}\right)-$ SGS electromotive force, tensor form

$\mathcal{E}=\overline{\mathbf{u} \times \mathbf{B}}-\tilde{\mathbf{u}} \times \overline{\mathbf{B}}-$ SGS electromotive force, vector form

$\epsilon$ - SGS energy dissipation

$\Sigma_{\square}$ - cross-scale transfer term, cascade term

$\mathcal{T}_{\square}^{\square}$ - non-local (diffusion-like) cross-scale transfer term, transport term

$\mathcal{F}_{\square}^{\square}$ - total cross-scale transfer term, total flux term

$C_{\square}$ - closure coefficient 


\section{Introduction}

\subsection{Fluid dynamics and MHD}

Understanding magnetised turbulent flows is an outstanding task for fluid dynamicists with applications from engineering to geophysics and astrophysics. Astrophysical plasmas, due to their typically low densities, tend to have small viscosity and hence exhibit strongly turbulent characteristics. Furthermore, as observations show, the universe has been fully ionized since redshift of $z \lesssim 5[27,32-35,49]$ and permeated by magnetic fields. While these magnetic fields are not always dynamically important, they feature as a probable ingredient in a multitude of outstanding astrophysical conundrums. At the largest scales, for example, the small-scale turbulent dynamo is a possible explanation for the amplification of the intracluster magnetic fields to the observed values of the order of $\mu G[19,53]$. At galactic scales, turbulent dynamos have been invoked to explain the presence of large-scale galactic magnetic fields [91]. Magnetohydrodynamic (MHD) turbulence also plays a significant role in the different phases of the ISM and may help explain the self-regulation of star formation as well as the fragmentation of pre-stellar cores. In stellar astrophysics MHD turbulence plays an important role in the convection zone and the photosphere of stars [7], in accretion and protoplanetary discs, for ejecta, e.g. jets, stellar outflows, etc. [11]. More locally - in solar physics, magnetised plasma determines the dynamics of the solar tachocline, the solar wind and the solar corona $[12,17,58,70,116]$. In the terrestrial environment examples can be found in different zones of the magnetosphere, for instance the geomagnetic tail $[13,39,47,94,115]$.

The parameter space which these phenomena occupy cannot be fully investigated in terrestrial experiments. The intrinsic non-linearity and large number of physical effects which they entail limits their analytical treatment as well. Numerical simulations have provided much insight into their dynamics due to completeness and flexibility of the provided datasets. However, the large dynamical range of scales coupled with nonlinear and compressibility effects render realistic astrophysical simulations prohibitively expensive in terms of computing resources. One method to reduce the computational cost is to simulate a limited range of scales, while the remaining, unresolved (i.e. not directly simulated) scales are modelled. This works focuses on one of the methods to do this - large eddy simulations (LES). After a brief introduction to MHD and LES, we derive a new model for the unresolved scales involved in LES in chapter 2. Other existing models available in literature are also described there. We then examine the properties of these unresolved scales as revealed by a set of high-resolution numerical simulations spanning a considerable range in parameter space in chapter 3 . Chapter 4 is dedicated to a comparison of the performance of the different models. Finally in 
chapter 5 we summarise the main results and provide a brief outlook.

\subsubsection{Magnetohydrodynamic equations}

The simplest approximation of describing both fluid motion and magnetic fields is magnetohydrodynamics (MHD), see e.g [84]. It is constructed by combining fluid dynamics (Navier-Stokes) and electromagnetism (Maxwell) under the approximation of a strongly collisional system with the following equations of motion:

$$
\begin{array}{r}
\frac{\partial \rho}{\partial t}+\nabla \cdot(\rho \mathbf{u})=0, \\
\frac{\partial \rho \mathbf{u}}{\partial t}+\nabla \cdot\left(\rho \mathbf{u} \otimes \mathbf{u}-\frac{\mathbf{B} \otimes \mathbf{B}}{4 \pi}\right)+\nabla\left(P+\frac{\mathbf{B}^{2}}{8 \pi}\right)=\nabla \cdot \sigma, \\
\frac{\partial \mathbf{B}}{\partial t}-\nabla \times(\mathbf{u} \times \mathbf{B})=\eta \nabla^{2} \mathbf{B}
\end{array}
$$

The viscous dissipation tensor is defined as $\sigma=2 \nu \mathcal{S}^{*}$, with $\mathcal{S}^{*}$ - the deviatoric (traceless) part of the kinetic rate-of-strain tensor $\mathcal{S}=\frac{1}{2}\left(\partial_{i} u_{j}+\partial_{j} u_{i}\right)$. The dynamic viscosity coefficient $\nu$ captures the strength of microphysical processes which dissipate kinetic energy into heat. Its counterpart in the induction equation is the Ohmic dissipation coefficient $\eta$. We can non-dimensionalize the system by introducing characteristic length, speed and density $V_{0}, L$ and $\rho_{0}$. Applying the transformation

$$
x \rightarrow x / L, \quad \rho \rightarrow \rho / \rho_{0}, \quad \mathbf{u} \rightarrow \mathbf{u} / V_{0}, \quad \mathbf{B} \rightarrow \mathbf{B} / B_{0}, \quad P \rightarrow P /\left(\rho_{0} V_{0}^{2}\right)
$$

with $B_{0}=V_{0} \sqrt{4 \pi \rho_{0}}$, we obtain the non-dimensional form of the equations of motion:

$$
\begin{aligned}
\frac{\partial \rho}{\partial t}+\nabla \cdot(\rho \mathbf{u}) & =0 \\
\frac{\partial \rho \mathbf{u}}{\partial t}+\nabla \cdot(\rho \mathbf{u} \otimes \mathbf{u}-\mathbf{B} \otimes \mathbf{B})+\nabla\left(P+\frac{\mathbf{B}^{2}}{2}\right) & =\frac{1}{\operatorname{Re}} \nabla \cdot\left(2 \mathcal{S}^{*}\right), \\
\frac{\partial \mathbf{B}}{\partial t}-\nabla \times(\mathbf{u} \times \mathbf{B}) & =\frac{1}{\mathrm{Rm}} \nabla^{2} \mathbf{B} .
\end{aligned}
$$

This gives the explicit definition of the kinetic Reynolds numbers as Re $=\rho_{0} V_{0} L / \nu$ and similarly the magnetic Reynolds number $-\mathrm{Rm}=V_{0} L / \eta$. The Reynolds numbers control the strength of the non-linear terms relative to the dissipation terms. Large Reynolds numbers (usually above $10^{3}$ ) are associated with turbulent flows. Additionally, we have the Alfvén speed - given by $v_{\mathrm{A}}=B / \sqrt{4 \pi \rho}$, which gives the Alfvénic Mach number $\mathrm{M}_{\mathrm{a}}=u / v_{\mathrm{A}}$. The latter signifies the strength of the magnetic field relative to the flow. Note that with our chosen system of units the initial Alfvénic Mach number is set to $\mathrm{M}_{\mathrm{a} 0}=1$. Similarly, we define the sonic Mach number to be $\mathrm{M}_{\mathrm{s}}=u / c_{s}$ for sound speed $c_{s}$.

The plasma beta parameter is an important MHD dimensionless quantity which gives a measure of the relative strength/dynamical importance of the velocity and magnetic field. It represents the ratio of thermal to magnetic pressures and can be expressed in 
terms of Mach numbers as $\beta_{\mathrm{p}}=2 \mathrm{M}_{\mathrm{a}}{ }^{2} / \mathrm{M}_{\mathrm{s}}{ }^{2}$. This parameter determines the importance of the "M" in MHD. The larger the parameter, the closer to the hydrodynamic limit we get.

To complete the set of equations we write down the expressions for the evolution of the kinetic energy $E^{\mathrm{u}}=\frac{1}{2} \rho \mathbf{u}^{2}$ and the magnetic energy $E^{\mathrm{b}}=\frac{1}{2} \mathbf{B}^{2}$

$$
\begin{array}{r}
\frac{\partial}{\partial t} E^{\mathrm{u}}+\nabla \cdot\left(\mathbf{u} E^{\mathrm{u}}\right)+\mathbf{u} \cdot \mathbf{B} \times \mathbf{J}+\mathbf{u} \cdot \nabla P=\frac{1}{\mathrm{Re}} \mathbf{u} \cdot \nabla \cdot\left(2 \mathcal{S}^{*}\right), \\
\frac{\partial}{\partial t} E^{\mathrm{b}}-\mathbf{B} \cdot \nabla \times(\mathbf{u} \times \mathbf{B})=\frac{1}{\mathrm{Rm}} \mathbf{B} \cdot \nabla^{2} \mathbf{B} .
\end{array}
$$

Combining them we obtain the total energy equation

$$
\begin{aligned}
\frac{\partial E}{\partial t}+\nabla \cdot\left(\mathbf{u} E^{\mathrm{u}}+2 \mathbf{u} E^{\mathrm{b}}-\mathbf{B} H\right) & = \\
-\mathbf{u} \cdot \nabla P & +\frac{1}{\mathrm{Re}} \mathbf{u} \cdot \nabla \cdot\left(2 \mathcal{S}^{*}\right)+\frac{1}{\mathrm{Rm}} \mathbf{B} \cdot \nabla^{2} \mathbf{B} .
\end{aligned}
$$

The right-hand side expresses dissipation into internal energy and can be closed via an equation of state. For the purposes of this analysis we limit ourselves to the simplest case of an isothermal equation of state $P \propto \rho$.

A new quantity enters the evolution equation, namely the the cross-helicity $H=\mathbf{u} \cdot \mathbf{B}$. It plays a crucial role in energy transport and conversion between kinetic and magnetic type. It describes the amount of (topological) twisting/linking between velocity and magnetic structures. Its evolution equation is a conservative one

$$
\frac{\partial}{\partial t} H+\nabla \cdot\left(\mathbf{u} H-\frac{\mathbf{B}}{\rho} E^{\mathrm{u}}\right)=-\frac{\mathbf{B}}{\rho} \cdot \nabla P+\frac{1}{\mathrm{Re}} \mathbf{B} \cdot \nabla \cdot\left(2 \mathcal{S}^{*}\right)+\frac{1}{\mathrm{Rm}} \mathbf{u} \cdot \nabla^{2} \mathbf{B} .
$$

In other words, this is another ideal MHD invariant along with the energy and the magnetic helicity (the latter will not be discussed in this work).

\subsubsection{Scale-separation: RANS, ILES, LES}

The analytical treatment of MHD turbulence is limited due to the strong non-linearities of the equations of motion. A numerical treatment, on the other hand, needs to resolve a huge dynamical range - from the microphysical scales where dissipation dominates the flow to the integral scales where the energy injection usually takes place. In 3 dimensions the computational cost scales with the $3^{\text {rd }}$ power of the largest of $\{\operatorname{Re}, R m\}$ (from Kolmogorov theory, the extent of the dynamical range in incompressible hydrodynamics (HD) scales with $R e^{3 / 4}$ per dimension: 3 spatial +1 temporal dimensions [66, p. 200], [25, p. 424] ). As an example [76], for the convection layer of our Sun, the Reynolds number has a value of $\operatorname{Re} \geq 10^{14}$. The most powerful high performing computers (HPC) today can perform at the level of pFLOPS $\left(10^{15}\right.$ floating point operations per second). The computation of only one dynamical time requires then more than the current age of the universe... 
In other environments the Reynolds numbers can reach even larger orders of magnitude (e.g. giant molecular clouds, intra-cluster medium). In addition astrophysical phenomena often occupy other extreme regions of parameter space with sonic and Alfvénic Mach numbers (ISM, outflows, novae) of order 10, large magnetic Prandtl numbers ${ }^{1}$ (stellar dynamos), etc. [70, 75]. Clearly some simplifications are required.

A promising way to proceed is to solve directly only the evolution of the large scales and to model the effect of the small-scales. This can only be feasible if there is some form of universality on the small scales so that a unifying model can be developed for more than one application at a time.

There are several approaches to reduce the computational cost and complexity. We will be concerned with large eddy simulations, but will mention the alternatives for completeness. They can be ordered by their level of simplification.

Reynolds averaged simulations (RANS) reduce the resolved degrees of freedom most drastically. There only the mean fields are computed, while all the fluctuations are subject to modelling. While they provide a significant reduction in computational cost, they are very sensitive to the particulars of the SGS model. Effectively they solve an extremely low effective Reynolds number approximation ${ }^{2}$.

A more expensive approach consists of the implicit LES (ILES). Here the assumption is that the numerical dissipation, which is inherent in any simulation, has the same effect as that of the SGS dynamics. This has the advantage that the SGS model is inherently stable and robust, by virtue of the properties of the numerical scheme. Additionally, it is computationally cheap, since it doesn't involve any additional computations. One disadvantage is that hydrodynamic ILES can suffer from a so-called bottleneck effect - a pile-up of energy at the end of the dissipation range [28, 79]. Additionally, by construction the SGS models are strictly dissipative, i.e. carrying energy only from large to small scales. However, it has been recorded (e.g. [79]) that there can be a non-negligible component of energy travelling in the opposite direction, which can be of the order of 10-20\% for hydrodynamic simulations and even dominant in MHD, e.g. in turbulent dynamo simulations with small initial magnetic field.

The LES approach consists of explicitly accounting for the effects of finite resolution in the evolution equations. While being more computationally expensive than ILES it provides a higher degree of control over the SGS dynamics and the flexibility to account for up-scale energy transfer. However it presents the closure problem - the need to develop an explicit model to describe these effects. This approach has already had great success in the fields of fluid dynamics and its engineering and astrophysical applications [51, 77, 79, 80]. However, only a few models have been developed in the context of compressible MHD (see chapter 2).

To illustrate the difference between RANS and LES we present fig. 1.1. It shows a turbulent field, its large scale, or 'resolved' component and the remaining fluctuations. In the RANS approach the field in fig. 1.1a needs to be modelled. In the LES approach we would have to model instead the field in fig. 1.1c. As can be seen, the latter is

\footnotetext{
${ }^{1}$ The magnetic Prandtl number is given by the ratio of microscopic viscosity and magnetic diffusivity as $P_{\mathrm{m}}=\nu / \eta=\mathrm{Rm} / \mathrm{Re}$.

${ }^{2}$ This effect comes from the fact that in low $\mathrm{Re}_{\text {effective }}$ simulations any fluctuations are quickly diffused away.
} 


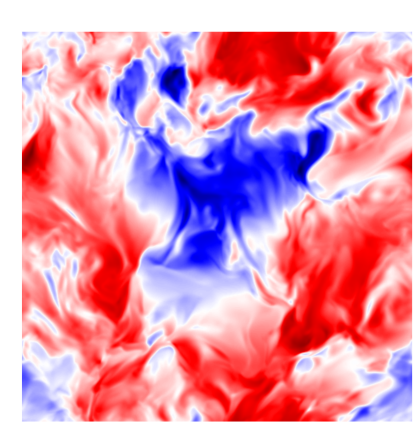

(a) full

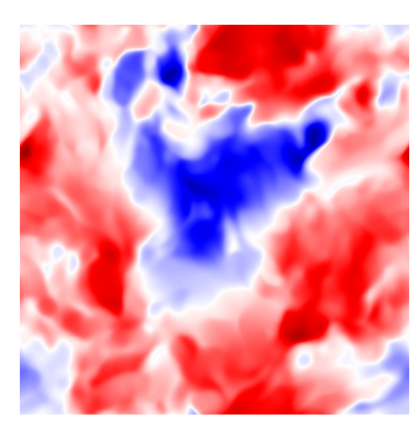

(b) 'resolved'

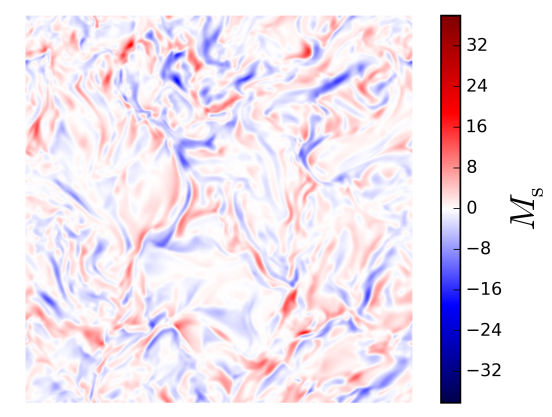

(c) fluctuations

Figure 1.1: Decomposition of a turbulent field (a) into its large scale 'resolved' (b) and small-scale - fluctuating (c) components. The illustration is of a slice of the z-component of the velocity field in units of the sound speed from a simulation of homogeneous and isotropic developed MHD turbulence.

in general much more homogeneous than the former and might exhibit some form of universality across different flows.

\subsection{Introduction to LES formalism}

\subsubsection{Filtering operator}

Let us then proceed with a brief overview of the LES formalism in its application to compressible MHD. For a comprehensive review of the formalism see e.g. [43, 74, 76] The effect of finite resolution in the MHD equations can be described by a low-pass filtering operator. In order to be useful for our purposes (i.e. application to partial differential equations) the filter kernel should satisfy a set of properties. It should preserve constants, commute with addition and with partial spatial and temporal derivatives. In other words the filtering operation is a convolution. This ensures that the LES equations are not drastically changed by the filtering operation. It can also describe any allowed discretization operator used in numerical simulations. We shall adopt the notation

$$
\bar{f}(\mathbf{x}, t)=G * f=\int_{\mathcal{D}, \mathcal{T}} G\left(\mathbf{x}-\boldsymbol{\xi}, t-t^{\prime}, \Delta, \tau_{0}\right) f\left(\boldsymbol{\xi}, t^{\prime}\right) \mathrm{d}^{3} \boldsymbol{\xi} \mathrm{d} t^{\prime}
$$

for a field $f$, filtered with filter kernel $G$ with spatial and temporal scales $\Delta$ and $\tau_{0}$, over a time interval $\mathcal{T}$ and spatial domain $\mathcal{D}$. The kernel $G$ is normalised by the preservation of constants condition $\bar{c}=c, \forall c \in \mathbb{R}$ to $\|G\|_{1}=1$.

Additionally, the filter kernel should have the symmetries of the MHD equations themselves, i.e. Galilean transformations, scaling, etc. This ensures the co-ordinate frame independence of the LES equations and results. Furthermore, in view of their applications to numerical simulations, time-independent filters are usually used. This simplifies the above expression to 


$$
\bar{f}(\mathbf{x}, t)=\int_{\mathcal{D}} G(|\mathbf{x}-\boldsymbol{\xi}|, \Delta) f(\boldsymbol{\xi}, t) \mathrm{d}^{3} \boldsymbol{\xi}
$$

Now, the fluid equations are non-linear, thus we need to define the commutator between filtering and multiplication. For two fields, $f$ and $g$, it is denoted by the second-order central moment $\mathcal{C}(f, g)=\overline{f g}-\bar{f} \bar{g}$. Figures $1.2 \mathrm{a}$ and $1.2 \mathrm{~b}$ demonstrate the importance of modelling $\mathcal{C}$ for a pair of real MHD fields.

It is noteworthy that, depending on the kernel, filtering doesn't need to be a Reynolds operator. Consider a field $f$ and its decomposition into filtered and unresolved components: $f=\bar{f}+f^{\prime}$. Then this statement is equivalent to $\overline{\bar{f}} \neq \bar{f}$ or $\overline{f^{\prime}} \neq 0$. This implies that the central moment doesn't contain information only about the small-scales but the large scales as well. To put it explicitly, $\overline{f g}=\overline{\bar{f}} \bar{g}+\overline{\bar{f} g^{\prime}}+\overline{f^{\prime} \bar{g}}+\overline{f^{\prime} g^{\prime}}$. So an alternative expression for $\mathcal{C}(x, y)$ is

$$
\mathcal{C}(x, y)=(\overline{\bar{f}} \bar{g}-\bar{f} \bar{g})+\left(\overline{\bar{f} g^{\prime}}+\overline{f^{\prime} \bar{g}}\right)+\overline{f^{\prime} g^{\prime}} .
$$

The three bracketed terms are known from left to right as the Leonard, cross and Reynolds terms. The Leonard term contains aliasing errors. The cross terms is responsible for the interaction of terms across the filter scale. The Reynolds terms deals with the self-interaction of the small scales and its effect on the large scales. Figures $1.2 \mathrm{c}-$ $1.2 \mathrm{e}$ show that each of the three components can be dominant in some region of the flow.

If the filtering is a Reynolds operator, i.e. idempotent, the second order moment reduces to products of filtered terms and filtered residuals, without mixed terms, i.e. $\overline{f g}=\bar{f} \bar{g}-\overline{f^{\prime} g^{\prime}}$. The idempotency in physical space is satisfied only by the trivial kernel $G=1$. In spectral space, it leads to a combination of Dirac delta and Heaviside step functions over non-intersecting domains [74]. This has a direct link with RANS and the wealth of results developed there and from statistical theory (e.g. [72, 109, 113, 114]) could be used directly in this context. Note that Reynolds operators reduce the number of degrees of freedom of the field due to its compact support in spectral space. On the other hand non-idempotent filters preserve the entire information of the filtered field, and thus can theoretically be inverted. This will lead to the main result of this work, first however we conclude this section with two additional desirable properties for the filtering operator.

If the filter kernel is positive, i.e. $G \geq 0, \forall x$, the filtering operation is signaturepreserving. This implies that if a quantity $h=f g$ is a positive semi-definite, then its SGS counterpart $h_{\mathrm{sgs}}=\overline{f g}-\bar{f} \bar{g}$ will also be a positive semi-definite. This will be useful in the interpretation and treatment of energy and pressure-like quantities at the sub-grid scales.

Finally, in certain large eddy simulations a hypothesis of scale-similarity is invoked. This requires the data to be explicitly filtered at run time. From computational standpoint it is then expedient if not necessary (depending on the boundary conditions) that the kernel is local, i.e has compact support, in physical or spectral space (depending on the type of numerical scheme under consideration). 


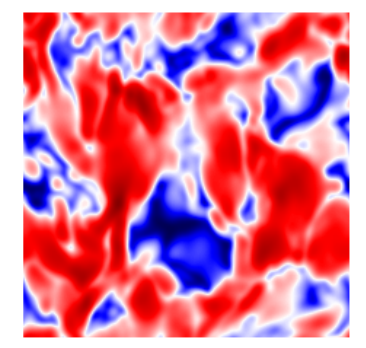

(a) full $-\overline{f g}$

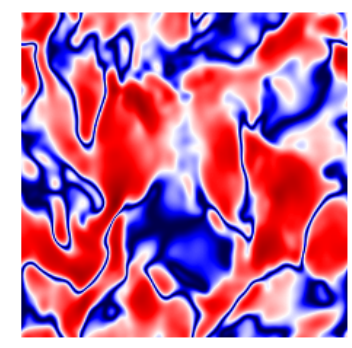

(b) 'resolved' - $\bar{f} \bar{g}$

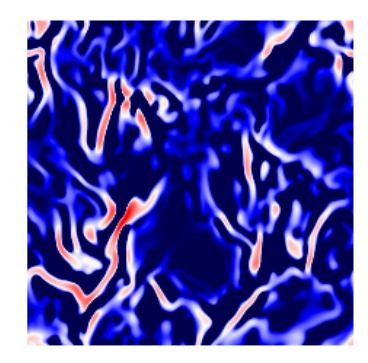

(c) Leonard $-\overline{\bar{f}} \bar{g}-\bar{f} \bar{g}$

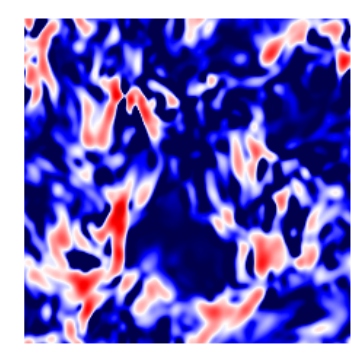

(d) $\operatorname{cross}-\overline{\bar{f} g^{\prime}}+\overline{f^{\prime} \bar{g}}$

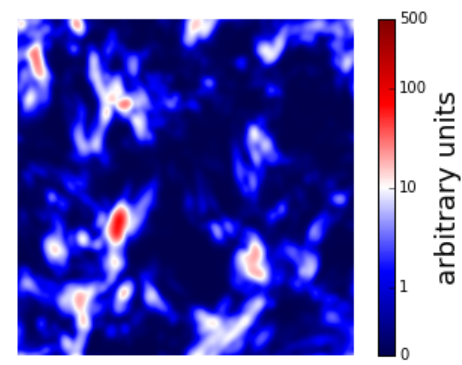

(e) Reynolds - $\overline{f^{\prime} g^{\prime}}$

Figure 1.2: Illustration of the difference between the different terms involved in a scale decomposition of a turbulent field $h=f g$.

\subsubsection{Filter selection and choice}

Most LES are approximated by one of a set of canonical filters: box, Gaussian, or sharp cut-off filters. The box filter constitutes a moving average over an interval with linear dimension $\Delta$. In Fourier space its kernel is represented by $\hat{G}=\operatorname{sinc}\left(k_{c}|\mathbf{x}-\boldsymbol{\xi}|\right)$, where $k_{c}=\pi / 2 \Delta$ is the cut-off wave number associated with the filtering scale $\Delta$. It is especially suited for grid-based simulations, especially finite volume ones. On the other hand, the sharp cut-off filter in Fourier space is a Heaviside step function $\Theta\left(k_{c}\right)$. It is particularly suited for spectral codes, for which it was developed, however problems are encountered when dealing with complex geometries and boundary conditions just as in the underlying spectral codes. Finally, the Gaussian filter is given by $\hat{G}=\exp \left(-\Delta^{2} k^{2} /(4 \gamma)\right)$ in Fourier space, where $\gamma$ is the normalisation parameter. For more details on the properties of these filters see [43]. A summary is given in tab. 1.1. It is important to note that they all have the necessary properties to maintain the symmetries of the MHD equations and thus lead to the same additional terms when applied to the MHD equations. The box and Gaussian filters are positive. The box filter is non-local in spectral space, leading to energy transfers outside the cascade paradigm ${ }^{3}$. The sharp filter is a Reynolds operator. This can simplify the modelling of the SGS terms tremendously. For example, it reduces or completely alleviates the need for struc-

\footnotetext{
${ }^{3}$ Briefly, the cascade paradigm stipulates that the transfer of energy takes place primarily between neighbouring Fourier modes. Thus the energy 'cascades' from the large injection scales to the small dissipation scales by passing through all the intermediate scales. This is the basis of the Kolmogorov theory.
} 
Table 1.1: Properties of filter kernel

\begin{tabular}{|l|l|l|l|}
\hline Property $\backslash$ Filter & Gaussian & Box & Sharp \\
\hline Galilean transformation & yes & yes & yes \\
\hline Derivative commutation & yes & yes & yes \\
\hline Positive & yes & yes & no \\
\hline Reynolds operator & no & no & yes \\
\hline Local in physical space & no & yes & no \\
\hline Local in spectral space & no & no & yes \\
\hline
\end{tabular}

tural modelling which deals primarily with the cross-terms (will be discussed below). However, as already discussed astrophysical application fair better with non-spectral codes, for example due to coupling with gravity/Poisson equation or to compressibility effects. Furthermore, the sharp filter is non-local in physical space and thus difficult to implement in self-similarity models. It is also not positive, so SGS energy- and pressure-like terms can take negative values, which hinders their interpretation and may induce artificial instabilities.

The Gaussian filter is somewhat intermediate between the box and sharp filters. It can be made to approximate either by adjusting the normalisation parameter $\gamma$. It is non-local in both physical and spectral space, however there are also computationally friendly approximations which render it local in physical space. Furthermore, because it is infinitely differentiable everywhere it facilitates analytical derivation and model building. This is why it will be used for the purposed of this study. We set the normalisation factor to $\gamma=6$, so that the second order moment matches that of the box filter.

\subsection{Magnetohydrodynamic equations for LES}

We proceed to apply the filtering formalism described above to the MHD equations (eqs. (1.5)-(1.7)). As we are interested in high Reynolds numbers applications we shall neglect the contributions of the dissipation terms in the primary equations and only consider them in the energy and cross-helicity equations for completeness.

The continuity equation is non-linear in the velocity and density, but we can use a trick to circumvent this complication - introduce mass-weighed filtering, i.e. $\widetilde{f}=$ $\overline{\rho f} / \bar{\rho}$. It was first introduced by [36] in order to retain the structure of the continuity equation and reduce the number of new, unknown terms in the filtered equations. With this in mind, convolving the MHD equations with the Gaussian kernel under periodic 
boundary conditions yields

$$
\begin{gathered}
\frac{\partial \bar{\rho}}{\partial t}+\nabla \cdot(\bar{\rho} \widetilde{\mathbf{u}})=0, \\
\frac{\partial \bar{\rho} \widetilde{\mathbf{u}}}{\partial t}+\nabla \cdot(\bar{\rho} \widetilde{\mathbf{u}} \otimes \widetilde{\mathbf{u}}-\overline{\mathbf{B}} \otimes \overline{\mathbf{B}})+\nabla\left(\bar{P}+\frac{\bar{B}^{2}}{2}\right)=-\nabla \cdot \tau, \\
\frac{\partial \overline{\mathbf{B}}}{\partial t}-\nabla \times(\widetilde{\mathbf{u}} \times \overline{\mathbf{B}})=\nabla \times \mathcal{E} .
\end{gathered}
$$

Here, $\bar{\rho}$ is the filtered density, $\overline{\mathbf{B}}$ the filtered magnetic field, $\bar{P}$ the filtered thermal pressure and $\tilde{\mathbf{u}}$ the mass-weighted filtered velocity. Two major new terms enter the set of equations: the SGS stress tensor $\tau$ and the electromotive force $(\mathrm{EMF})^{4} \mathcal{E}$. Their analytical expressions are given by

$$
\begin{gathered}
\mathcal{E}=\overline{\mathbf{u} \times \mathbf{B}}-\tilde{\mathbf{u}} \times \overline{\mathbf{B}} \\
\tau_{i j}=\bar{\rho}\left(\widetilde{u_{i} u_{j}}-\widetilde{u}_{i} \widetilde{u}_{j}\right)-\left(\overline{B_{i} B_{j}}-\bar{B}_{i} \bar{B}_{j}\right)+\frac{1}{2}\left(\overline{B^{2}}-\bar{B}^{2}\right) \delta_{i j} .
\end{gathered}
$$

These terms are not expressed in terms of resolved fields and thus render the system of equations unclosed. We could write down their evolution equations. However, they involve third-order moments (see e.g. sections 3.4 and 3.5), which are also unknown and so on to build an infinite hierarchy. This is usually referred to as the closure problem. One truncates the hierarchy usually at the level of $\tau$ and $\mathcal{E}$ by providing expressions in terms of known variables, i.e. closures.

The SGS stress is naturally decomposed into its kinetic (the SGS Navier-Stokes term) and magnetic (the SGS Lorentz term) components. We define

$$
\begin{aligned}
\tau_{i j}^{\mathrm{u}} & \equiv \bar{\rho}\left(\widetilde{u_{i} u_{j}}-\widetilde{u}_{i} \widetilde{u}_{j}\right) \\
\tau_{i j}^{\mathrm{b}} & \equiv\left(\overline{B_{i} B_{j}}-\bar{B}_{i} \bar{B}_{j}\right)-\frac{1}{2}\left(\overline{B^{2}}-\bar{B}^{2}\right) \delta_{i j}
\end{aligned}
$$

Their isotropic parts have the form of SGS kinetic and magnetic energies, i.e. the difference between the energy on the filter scale and the energy contained only in the resolved fields.

$$
\begin{gathered}
E_{\mathrm{sgs}}^{\mathrm{u}}=\frac{1}{2} \tau_{k k}^{\mathrm{u}}=\frac{1}{2} \bar{\rho}\left(\widetilde{u^{2}}-\widetilde{u}^{2}\right) \\
E_{\mathrm{sgs}}^{\mathrm{b}}=\tau_{k k}^{\mathrm{b}}=\frac{1}{2}\left(\overline{B^{2}}-\bar{B}^{2}\right)
\end{gathered}
$$

However, in order to be interpreted as energies they need to be positive definite, which is only guaranteed for a positive filter (as discussed in the previous section). This is one of the major reasons to disfavour the sharp spectral filter. We reiterate that these energies are associated not only with the small-scale fluctuations but also with cross-scale interactions and large-scale aliasing corrections.

\footnotetext{
${ }^{4}$ As a technical aside, the electromotive force $\mathcal{E}$ is a pseudo-vector and thus can be re-written as an anti-symmetric tensor of the form $\mathcal{E}_{i j} \equiv \epsilon_{i j k}(\mathcal{E})_{k}$. The inverse identity reads $(\mathcal{E})_{k} \equiv \frac{1}{2} \epsilon_{i j k} \mathcal{E}_{i j}$.
} 
The total SGS stress can be finally be decomposed as

$$
\tau_{i j}=\tau_{i j}^{\mathrm{u} *}-\tau_{i j}^{\mathrm{b} *}+2 E_{\mathrm{sgs}}^{\mathrm{u}}+E_{\mathrm{sgs}}^{\mathrm{b}} .
$$

We shall refer to the total isotropic term as the SGS pressure $P_{\mathrm{sgs}}=2 E_{\mathrm{sgs}}^{\mathrm{u}}+E_{\mathrm{sgs}}^{\mathrm{b}}$ since it enters the momentum equation in the same way as the thermal pressure $P$ and has a SGS origin.

Next we consider how filtering affects the energy and cross-helicity. Since the proper treatment of dissipation is crucial here, we re-introduce the dissipative terms in a summary form (denoted by $\epsilon$ ). Since they dominate on scales below our filter scale, we shall keep them primarily for book keeping and leave a detailed study of their influence on LES for future investigation.

There are three forms of the energy and cross-helicity for which we can write evolution equations in the context of LES. First, there are the quantities at the filter scale. They are obtained by directly filtering the corresponding MHD equation

$$
\begin{array}{r}
\frac{\partial}{\partial t} \bar{E}^{\mathrm{u}}+\nabla \cdot\left(\overline{\mathbf{u} E^{\mathrm{u}}}\right)+\overline{\mathbf{u} \cdot \mathbf{B} \times \mathbf{J}}+\overline{\mathbf{u} \cdot \nabla P}=\epsilon_{\mathrm{u}}^{\mathrm{ft}}, \\
\frac{\partial}{\partial t} \bar{E}^{\mathrm{b}}-\overline{\mathbf{B} \cdot \nabla \times(\mathbf{u} \times \mathbf{B})}=\epsilon_{\mathrm{b}}^{\mathrm{ftt}}, \\
\frac{\partial}{\partial t} \bar{H}+\nabla \cdot \overline{\left(\overline{\mathbf{u} H}-\overline{\mathbf{B}} \frac{\bar{\rho}}{\rho}\right)+\overline{\mathbf{B}} \cdot \nabla P}=\epsilon_{\mathrm{ch}}^{\mathrm{ftt}} .
\end{array}
$$

Note that the quantities under the divergence and filtering operators are products of three primary fields. This illustrates the closure problem - the evolution of second order quantities contains unclosed terms of third order. Combining eq. (1.22) with eq. (1.23) we obtain the compact expression for the total energy at the filter scale $\bar{E}$

$$
\frac{\partial \bar{E}}{\partial t}+\nabla \cdot\left(\overline{\mathbf{u} E^{\mathrm{u}}}+2 \overline{\mathbf{u} E^{\mathrm{b}}}-\overline{\mathbf{B} H}\right)+\overline{\mathbf{u} \cdot \nabla P}=\epsilon_{\mathrm{en}}^{\mathrm{ft}}
$$

Clearly, this is an ideal MHD invariant (allowing for the evolution of the internal energy through the thermal pressure $P$ ). The terms $\epsilon_{\square}^{\mathrm{ftt}}$ encode the total dissipation into microscopic degrees of freedom.

In LES we only have access to resolved quantities, namely

$$
E_{\mathrm{res}}^{\mathrm{u}}=\frac{1}{2} \bar{\rho} \tilde{\mathbf{u}}^{2}, E_{\mathrm{res}}^{\mathrm{b}}=\frac{1}{2} \overline{\mathbf{B}}^{2}, E_{\mathrm{res}}=E_{\mathrm{res}}^{\mathrm{u}}+E_{\mathrm{res}}^{\mathrm{b}}, H_{\mathrm{res}}=\tilde{\mathbf{u}} \cdot \overline{\mathbf{B}}
$$

Their evolution equations are obtained from primary LES equations by a scalar product with the appropriate resolved field (e.g. the resolved kinetic energy $E_{\text {res }}^{\mathrm{u}}$ is obtained 
from the inner product of eq. (1.14) with $\tilde{\mathbf{u}}$ ). The full equations are given by

$$
\begin{aligned}
\frac{\partial}{\partial t} E_{\mathrm{res}}^{\mathrm{u}}+\nabla \cdot\left(\tilde{\mathbf{u}} E_{\mathrm{res}}^{\mathrm{u}}\right)+\tilde{\mathbf{u}} \cdot \overline{\mathbf{B}} \times \overline{\mathbf{J}}+\tilde{u} \cdot \nabla \bar{P} & =-\tilde{u} \cdot(\nabla \cdot \tau)+\epsilon_{\mathrm{u}}^{\mathrm{res}} \\
\frac{\partial}{\partial t} E_{\mathrm{res}}^{\mathrm{b}}-\overline{\mathbf{B}} \cdot \nabla \times(\tilde{\mathbf{u}} \times \overline{\mathbf{B}}) & =\bar{B} \cdot \nabla \times \mathcal{E}+\epsilon_{\mathrm{b}}^{\mathrm{res}} \\
\frac{\partial E_{\mathrm{res}}}{\partial t}+\nabla \cdot\left(\tilde{\mathbf{u}} E_{\mathrm{res}}^{\mathrm{u}}+2 \tilde{\mathbf{u}} E_{\mathrm{res}}^{\mathrm{b}}-\overline{\mathbf{B}} H_{\mathrm{res}}\right)+\tilde{\mathbf{u}} \cdot \nabla \bar{P} & =\bar{B} \cdot \nabla \times \mathcal{E}-\tilde{u} \cdot(\nabla \cdot \tau)+\epsilon_{\mathrm{en}}^{\mathrm{res}} \\
\frac{\partial}{\partial t} H_{\mathrm{res}}+\nabla \cdot\left(\tilde{\mathbf{u}} H_{\mathrm{res}}-\frac{\overline{\mathbf{B}}}{\bar{\rho}} E_{\mathrm{res}}^{\mathrm{u}}\right)+\frac{\overline{\mathbf{B}}}{\bar{\rho}} \cdot \nabla \bar{P} & =\tilde{\mathbf{u}} \cdot \nabla \times \mathcal{E}-\frac{\overline{\mathbf{B}}}{\bar{\rho}} \cdot(\nabla \cdot \tau)+\epsilon_{\mathrm{ch}}^{\mathrm{res}} .
\end{aligned}
$$

Here even in the ideal case the conservative form is lost due the SGS terms on the right hand side. They incorporate the transfer across the filter scale. The $\epsilon_{\square}^{\text {res }}$ terms, on the other hand, are responsible for the direct dissipation from resolved to microscopic scales. Within the cascade paradigm the (spectrally) local transfers associated with the SGS terms are preferred and thus the $\epsilon$-terms are sub-dominant. This is one reason why they can often be neglected in LES. Note that there are no new unclosed terms coming from these equations, i.e. closing the primary equations automatically closes these ones as well. In other words, the effect of the unresolved scales on the resolved energies and cross-helicity is entirely contained within the SGS stress and EMF.

What we lack in an LES simulation is the difference between the total and resolved quantities, namely the SGS energies and cross-helicity. They balance the extra source terms in the resolved equations to maintain the total invariance. We have already defined the SGS energies from the traces of the kinetic and magnetic SGS stress. The SGS cross-helicity has the analogous form $H_{\mathrm{sgs}}=\overline{\mathbf{u} \cdot \mathbf{B}}-\tilde{\mathbf{u}} \cdot \overline{\mathbf{B}}$. Their evolution equations are obtained simply by subtracting the resolved eqs. (1.26)-(1.29) from the equations for the total terms - eqs. (1.22)-(1.25). The result is

$$
\begin{aligned}
\frac{\partial}{\partial t} E_{\mathrm{sgs}}^{\mathrm{u}}+\mathcal{F}_{3}^{\mathrm{u}}+\mathcal{F}^{\mathrm{en}, \text { ther }} & =\tilde{u} \cdot(\nabla \cdot \tau)+\epsilon_{\mathrm{sgs}}^{\mathrm{u}}, \\
\frac{\partial}{\partial t} E_{\mathrm{sgs}}^{\mathrm{b}}+\mathcal{F}_{3}^{\mathrm{b}} & =-\bar{B} \cdot \nabla \times \mathcal{E}+\epsilon_{\mathrm{sgs}}^{\mathrm{b}}, \\
\frac{\partial}{\partial t} E_{\mathrm{sgs}}+\mathcal{F}_{3}^{\mathrm{en}}+\mathcal{F}^{\mathrm{en}, \text { ther }} & =\tilde{u} \cdot(\nabla \cdot \tau)-\bar{B} \cdot \nabla \times \mathcal{E}+\epsilon_{\mathrm{sgs}}^{\mathrm{en}}, \\
\frac{\partial}{\partial t} H_{\mathrm{sgs}}+\mathcal{F}_{3}^{\mathrm{ch}}+\mathcal{F}^{\mathrm{ch}, \text { ther }} & =-\tilde{\mathbf{u}} \cdot \nabla \times \mathcal{E}+\frac{\overline{\mathbf{B}}}{\bar{\rho}} \cdot(\nabla \cdot \tau)+\epsilon_{\mathrm{sgs}}^{\mathrm{ch}}
\end{aligned}
$$

Here we introduce the flux terms $\mathcal{F}_{3}^{\square}$, which contain third-rank unclosed terms of the form $\overline{f g h}-\bar{f} \bar{g} \bar{h}$ (or its Favre variation) and the thermal pressure flux terms ${ }^{5}$ $\mathcal{F}^{\square \text {,ther }}$, whose closure depends on the adopted equation of state. We shall discuss their detailed form, structure and function in chapter 3. For now we just point out

\footnotetext{
${ }^{5}$ Sometimes known as dilatation terms in the energy equation
} 
that since they don't enter the equations of the resolved terms they don't directly participate in the transfer across the filter scale or affect directly the resolved primary fields. The right hand side of the equations is the focus of most models. The dissipation terms $\epsilon_{\square}^{\text {sgs }}$ contain the final part of the cascade from the unresolved continuum to the microscopic, kinetic scales. Since they contain the least amount of energy/cross-helicity their closure is usually neglected in favour of the familiar second order SGS terms $\tau$ and $\mathcal{E}$, eventhough theoretically, the $\epsilon$-terms should determine the slope of the entire spectrum and the cascade speed and efficiency.

\subsection{Validation methods}

Before we proceed with the description of the closures for the unknown SGS terms, let us consider the criteria by which to gauge the success of our modelling attempts. There are two main approaches to this problem - a priori and a posteriori (as coined in [65]). In both case one has to choose a set of diagnostic parameters for testing.

In the a posteriori method one implements an SGS model directly in an LES simulation and compares the results to reliable data (higher resolution DNS, experimental data, etc.). Usually sensitive parameter statistics are chosen for a posteriori validation, e.g. $\sqrt{\left\langle\rho^{\prime 2}\right\rangle}$ [85], energy spectra, time evolution of plasma parameters statistics (like Re, $\mathrm{M}_{\mathrm{a}}$, etc.).

In a priori tests, we take data from a high resolution simulation ${ }^{6}$, filter it explicitly with a test filter and obtain exact expressions for the sub-test-filter terms. These expressions can then be compared with the respective SGS models based on supertest-filter fields. To be precise, in this case there are two levels of smoothing: the grid scale of the original simulation $\Delta_{\mathrm{g}}$ and the scale of the test filter $\Delta_{\mathrm{t}}$. Thus the sub-test-filter quantities only refer to the scale range between $\Delta_{\mathrm{t}}$ and $\Delta_{\mathrm{g}}$. However, as long as $\Delta_{\mathrm{g}} \ll \Delta_{\mathrm{t}}$, and the smallest scales are energetically suppressed, this distinction can be ignored ([79]). Thus we shall refer to the the sub-test-filter terms as simply unresolved or SGS terms. Furthermore, for statistically stationary, homogeneous and isotropic turbulence, the closure coefficients ${ }^{7}$ are independent of the filter scale (within the inertial range due to the equilibrium of energy transfer in the inertial range) so the coefficients derived at scale $\Delta_{\mathrm{t}}$ are valid for $\Delta_{\mathrm{g}}$ as well ([77]). The main challenge here is the comparison between the 'true' SGS terms and their respective models. Usually a set of diagnostic parameters is chosen, which preferably reduce the dimensionality of the SGS terms and thus avoid dependence on the co-ordinates, i.e. the frame of reference. To this effect the SGS terms are contracted with various tensors based on different motivations - statistical, dynamical, geometrical, etc.

In this work we take the a priori approach and focus on energetic and geometrical diagnostics. In the following section we shall describe the details of the data which will

\footnotetext{
${ }^{6}$ Trivially, the simulation should be run without an SGS model implementation. Furthermore, high resolution here means that a scale-independent, power-law range in the energy power spectrum can be clearly identified.

${ }^{7}$ We shall see in the following chapter that most models come with a set of free coefficients which may need to be determined externally.
} 
be analysed.

\subsection{Introduction of sample data}

Since experimental data is not available for the parameter space we are concerned with, we have to rely on numerical simulations. To reduce systematic errors from code structure we use data obtained by two astrophysical MHD codes: FLASHV4 [41] and ENZO [92].

We begin with a few words about the codes themselves. Both ENZO and FLASHv4 are finite-volume grid-based codes solving the equations of motion (mass, momentum, energy, magnetic field) in integral form, thus preserving the constants of motion to machine precision. ENZO solves the ideal MHD equations by a second order accurate Godunov extension - MUSCL-Hancock scheme [100], with second-order Runge-Kutta time integration. We use two of the available Riemann solvers: a Harten-Lax-van Leer (HLL) Riemann - two-wave, three-state solver and HLLD - five-wave solver (HLL with multiple discontinuities [92]). FLASHv4 has similar accuracy to ENZO, but uses the positive-definite HLL3R Riemann solver and can handle explicit viscosity and resistivity. Both codes employ divergence cleaning [26] to maintain the solenoidal condition for the magnetic field $\nabla \cdot B=0$.

\subsubsection{Analysed parameter space}

We will analyse simulations of driven MHD turbulence on a periodic cubic grid of length $L$ with linear resolution of 512 . The driving is stochastic, statistically homogeneous and isotropic (similar to [38, 78]). It is implemented only in the momentum equation via a random Ornstein-Uhlenbeck process at small wave numbers. In spectral space it has a parabolic profile between $1<|\mathbf{k}| L / 2 \pi<3$ with a peak at $\mathbf{k} \mid L / 2 \pi=2$. It evolves with time and space. Its auto-correlation time $T$ is associated with the turnover of the largest energy-containing eddies. Thus it is the chosen time unit for the rest of this work. Table 1.2 contains the key plasma parameters and simulation details for the dataset under consideration.

We use a combination of solenoidal and compressive forcing, as measured by the compressive ratio $\zeta=\frac{(\nabla \cdot \mathbf{u})^{2}}{(\nabla \cdot \mathbf{u})^{2}+(\nabla \times \mathbf{u})^{2}}$. In the majority of the runs the components have equal weight(i.e. $\zeta=0.5$ ), however for a subset we increase the compressive component to $\zeta=0.9([37,79])$. These choices result in the combination of solenoidal and compressive velocity modes described by fig. 1.3. Here, in addition to $\zeta$ we show the mass-weighted distribution of solenoidal versus compressive modes, as it may better capture the effect of compressibility through density variations.

The forcing amplitude effectively determines the saturated sonic Mach number - fig. 1.4. To maintain frame independence we show its root-mean-square (RMS) value. We explore a range of RMS Mach numbers from the subsonic to the highly supersonic regime. We see that the Mach number values are saturated and statistically stationary for $T>2$. Thus we shall focus the bulk of the analysis on that part of the simulations and refer to it as well-developed or statistically stationary turbulent regime. The initial 


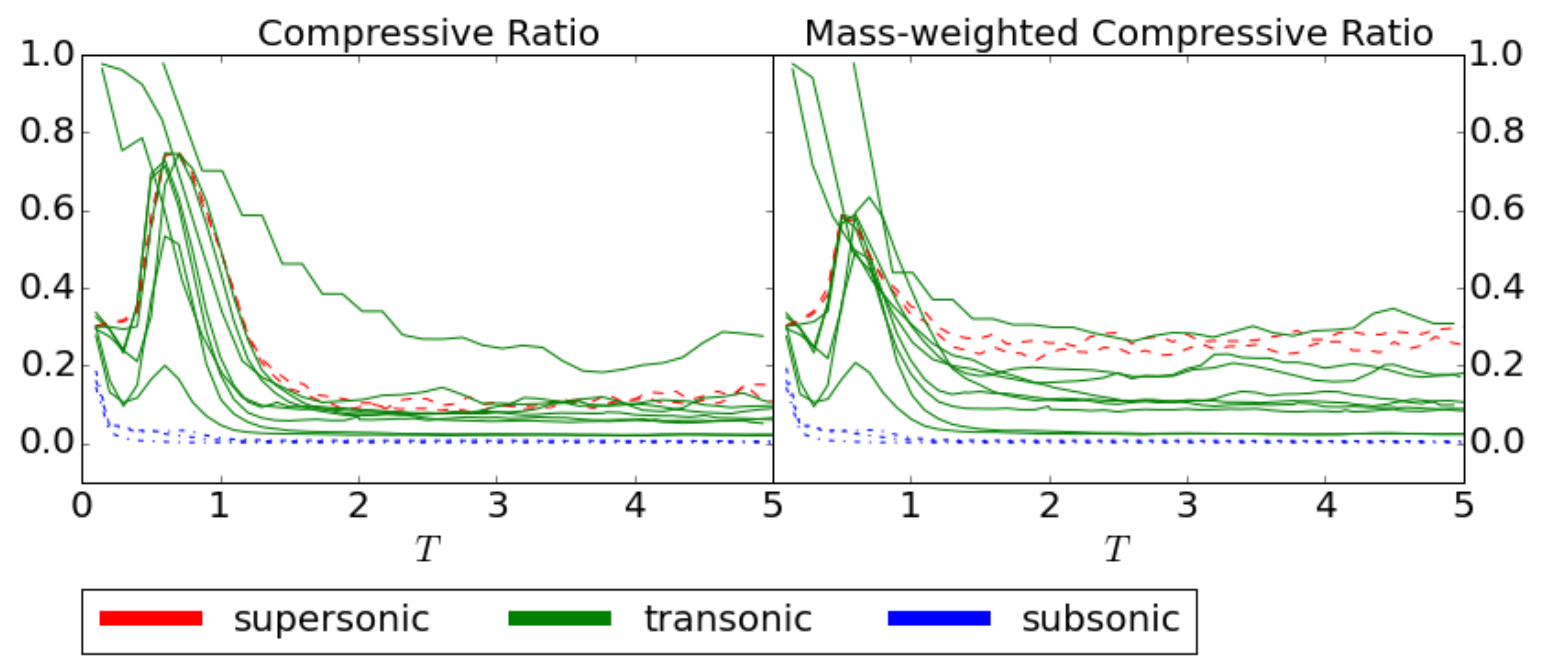

Figure 1.3: The time evolution of the compressive ratio for the velocity field, given by the spatial average of $\zeta$ in the left panel and the spatial average of the mass-weighted version of $\zeta$ in the right panel for each simulation from table 1.2. The colours represent the RMS sonic Mach number regimes: blue for subsonic, green for transonic and red for supersonic.

phases could be investigated with regards to small-scale dynamo processes, however they are also strongly dependent on the particulars of the initial conditions and the forcing. Hence interpretations drawn from them are not as robust with respect to different flow configurations, e.g. different initial or boundary conditions.

The initial conditions are: constant mass density, zero velocity and uniform magnetic field along the z-axis. The strength of the initial magnetic field is given by the initial plasma beta parameter $\beta_{\mathrm{p}, 0}$.

The FLASHv4 simulations use a polytropic equation of state with adiabatic index $\gamma=1$ which renders the simulations exactly isothermal. In ENZO this option is not available, so we use the approximation of an ideal gas law with adiabatic index $\gamma=$ 1.001. This allows us to neglect the heating and cooling effects in these runs as well. For astrophysical plasma applications this is a significant simplification. However, this work aims simply to gauge the performance of the closures under consideration. Further tests in more realistic simulations can be performed for models which satisfy the criteria of this study.

The clearest indication of the presence and strength of turbulence in the simulations is given by the Reynolds numbers. In the computational context the effective Reynolds numbers can be defined $[40,66]$ on the grid scale as $\operatorname{Re}=2 L^{2}\left\langle|\boldsymbol{\Omega}|^{2}\right\rangle /\left\langle\mathbf{V}^{2}\right\rangle$ and $R \mathrm{~m}=2 L^{2}\left\langle\left|\mathbf{J}^{2}\right|\right\rangle /\left\langle\mathbf{B}^{2}\right\rangle$. For the considered simulations they are of the order of $10^{3}$ (see fig. 1.5) which is far from the astrophysical regime, but is sufficient to sustain turbulence. This represents the strength of the fluctuations on the grid scale. The respective viscosity/resistivity could be calculated from the definition of the Reynolds numbers. However, this would invoke the assumption of constant viscosity/diffusivity with respect to space, which is not satisfied on the grid scale. 


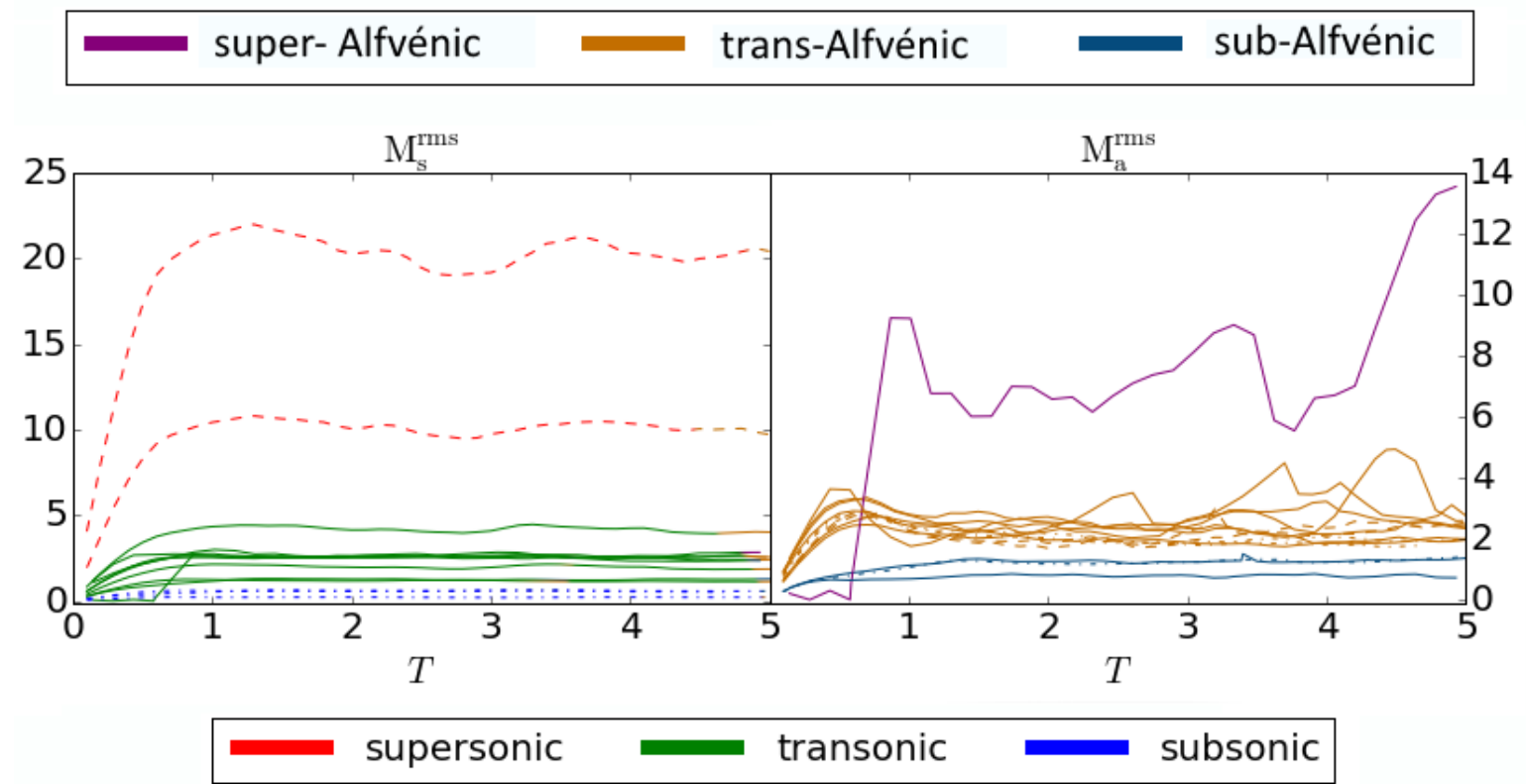

Figure 1.4: The time evolution of the root-mean-square (RMS) sonic (left panel) and Alfvénic (right panel) Mach numbers for each simulation from table 1.2. In the left panel the colours distinguish the RMS sonic Mach number regimes: blue for subsonic, green for transonic and red for supersonic. In the right panel the colours distinguish the RMS Alfvénic Mach number regimes: purple for super-Alfvénic, ochre for trans-Alfvénic and blue for sub-Alfvénic.

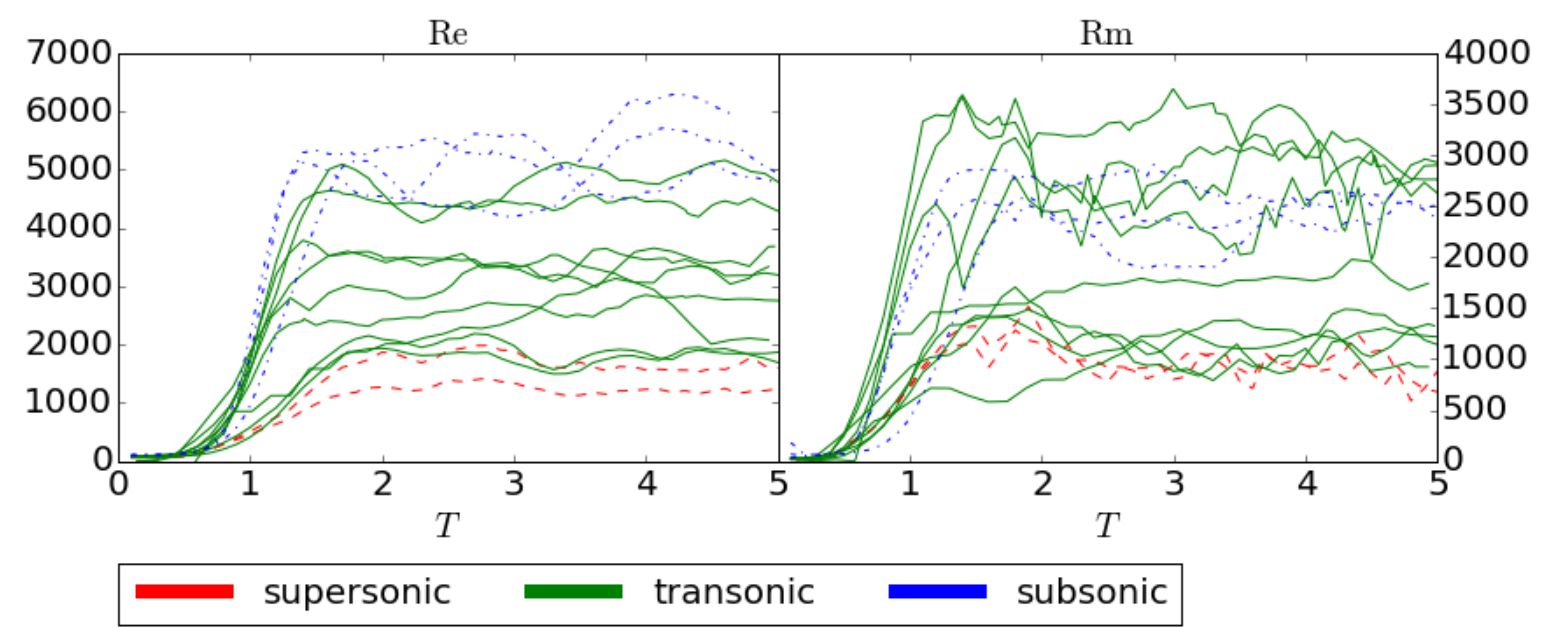

Figure 1.5: The time evolution of the effective Reynolds numbers on the grid scale (of $512^{3}$ ) (Re in left panel, Rm in right panel) for each simulation from table 1.2. The colours represent the RMS sonic Mach number regimes: blue for subsonic, green for transonic and red for supersonic. 
Table 1.2: Parameters of the a priori simulations

\begin{tabular}{|c|c|c|c|c|c|c|c|c|c|c|}
\hline Sim. \# & Driving $\mathrm{M}_{\mathrm{s}}$ & $\beta_{\mathrm{p}, 0}$ & Eqn. of State & $\left\langle\mathrm{M}_{\mathrm{s}}\right\rangle$ & $\left\langle\mathrm{M}_{\mathrm{a}}\right\rangle$ & Re & Rm & Code & Solver & Compressive ratio \\
\hline 1 & 0.2 & 450 & $e=\frac{p}{1-1.001}$ & 0.21 & 1.35 & - & - & ENZO & HLLD & 0.5 \\
\hline 2 & 0.5 & 72 & $e=\frac{p}{1-1.001}$ & 0.51 & 1.25 & - & - & ENZO & HLLD & 0.5 \\
\hline 3 & 0.5 & 8 & $e=\frac{p}{1-1.001}$ & 0.55 & 0.92 & - & - & ENZO & HLLD & 0.5 \\
\hline 4 & 1 & 18 & $e=\frac{p}{1-1.001}$ & 1.07 & 1.23 & - & - & ENZO & HLLD & 0.5 \\
\hline 5 & 1 & 2 & $e=\frac{p}{1-1.001}$ & 1.15 & 0.94 & - & - & ENZO & HLLD & 0.5 \\
\hline 6 & 2 & 5 & $P=\rho$ & 1.79 & 1.38 & 3780 & 3780 & FLASHv4 & HLL3R & 0.5 \\
\hline $7 \mathrm{a}$ & 2 & 5 & $e=\frac{p}{1-1.001}$ & 2.42 & 1.50 & - & - & ENZO & HLL & 0.5 \\
\hline $7 \mathrm{~b}$ & 2 & 5 & $e=\frac{p}{1-1.001}$ & 2.33 & 1.44 & - & - & ENZO & HLLD & 0.5 \\
\hline 8 & 2.9 & 25 & $e=\frac{p}{1-1.001}$ & 2.35 & 3.44 & - & - & ENZO & HLL & 0.9 \\
\hline 9 & 2.9 & 2.5 & $e=\frac{p}{1-1.001}$ & 2.35 & 1.34 & - & - & ENZO & HLL & 0.9 \\
\hline 10 & 2.9 & 0.25 & $e=\frac{p}{1-1.001}$ & 2.40 & 0.56 & - & - & ENZO & HLL & 0.9 \\
\hline 11 & 4 & 1 & $P=\rho$ & 3.77 & 1.18 & 3780 & 3780 & FLASHV4 & HLL3R & 0.5 \\
\hline 12 & 10 & 0.2 & $P=\rho$ & 9.14 & 1.01 & 3780 & 3780 & FLASHV4 & HLL3R & 0.5 \\
\hline 13 & 20 & 0.05 & $P=\rho$ & 19.43 & 0.97 & 3780 & 3780 & FLASHV4 & HLL3R & 0.5 \\
\hline
\end{tabular}

The expressions $\left\langle\mathrm{M}_{\mathrm{s}}\right\rangle$ and $\left\langle\mathrm{M}_{\mathrm{a}}\right\rangle$ refer to averages over the simulation domain and time period in which a statistically stationary state is reached. 


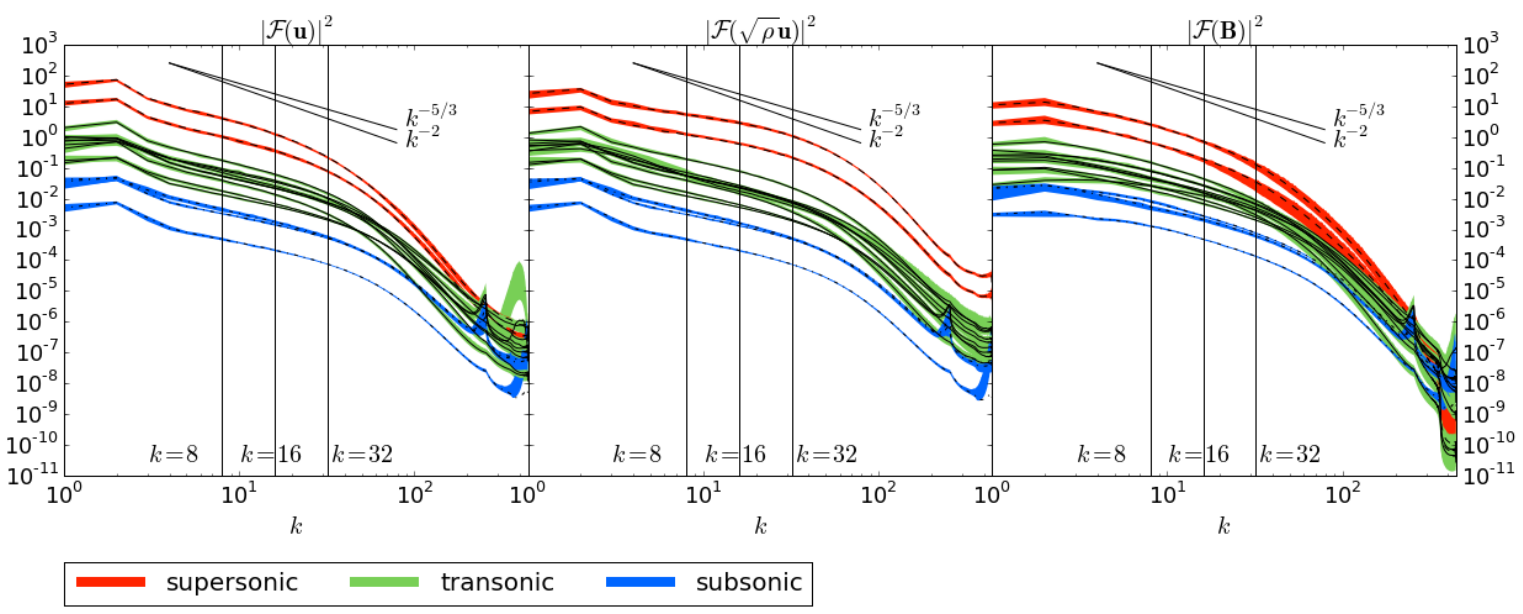

Figure 1.6: Spectra of the velocity, mass-weighted velocity and magnetic field for each simulation from table 1.2. The lines represent the median spectra with respect to the time period $2 \leq T \leq 5$ and the encompassing shaded regions represent the extent of the time variation. The colours represent the RMS sonic Mach number regimes: blue for subsonic, green for transonic and red for supersonic.

\subsubsection{Choice of filter scale}

In order to proceed with a priori analysis of the data, we need to choose a scale at which to apply the explicit filter, in other words to fix the parameter $\Delta$. In general for smooth filters, like the Gaussian, the interpretation of such a scale is somewhat arbitrary, but loosely it is a scale beyond which the power of the modes is strongly attenuated. Its position carries physical implications. If it is placed well within the dissipation range, that implies that the turbulence cascade is well resolved and the modelling needs to be done solely for dissipation and not turbulence effects. Conversely, retaining only low resolution, i.e. placing the filter scale in the integral range, means that we are approaching the RANS regime, where only the mean flow is solved for and all perturbations are modelled. In this case some of the integral dynamics have to be incorporated in the SGS model - i.e. the model becomes dependent on the specifics of the initial and boundary conditions and the forcing, so it is not universal and has to be adapted for every new simulation conditions. Additionally, since the whole inertial and dissipation range has to be modelled one needs an already complete turbulent theory, which is unfortunately not available.

In fig. 1.6, we consider the spectra of the velocity $\mathbf{u}$, mass-weighted velocity $\sqrt{\rho} \mathbf{u}$ and the magnetic field $\mathbf{B}$ for all simulations. We consider the mass-weighted velocity as a more appropriate representative of the cascade-bearing properties for compressible turbulence. As we can see from the figures the power-law range extends over a bit more than a decade and thus the simulations are sufficiently well resolved to be suitable for $a$ priori analysis. As an aside, the $\mathbf{u}$-based spectra are close to the classical Kolmogorov (incompressible) $-5 / 3$ and Burgers (compressible) -2 scaling of hydrodynamic turbulence; we get shallower profiles with the stronger driving. The mass-weighted velocity spectra all exhibit significantly shallower slopes. 
Ideally the cut-off scale should be placed well within the inertial range far away from numerical effects at both large and small scales. The features and even existence of a classical inertial range in MHD has been questioned (e.g. [2, 3, 8, 42]). However, for brevity, we shall use this term to refer to the scales which exhibit an approximate power-law in the energy spectrum as this is a signature of the self-similarity property of the classical turbulent fluctuations.

The most widely used criterion for the placement of filter scale is the middle of the inertial range [45] - this guarantees that there is turbulent dynamics on both sides of the filter. This allows a scale-similarity hypothesis to be invoked for model coefficient estimation. A lower bound for the filter scale is given by [85]. They maintain that the filter should be placed at a place where the truncation error is small. The main point being that at the Nyquist scale $k_{N y}$ the numerical simulation incurs a truncation error of order unity, so placing the filter too close to $k_{N y}$ strongly contaminates the a priori results. They use a safety factor of $2 / 3 k_{N y}$.

We employ several filter scales $\Delta=8,16,32$. The bulk of the analysis, will be focused on results obtained from filter scale $\Delta=16$ which corresponds to wavenumber $k=16$. This falls well within the inertial part of the spectrum as can be seen in fig. 1.6. The other two filter scales lie at the outskirts of the inertial range where the influence from forcing or dissipation effects begins to become significant. Comparing the results at different filter scales will shed light on the applicability of the various models to limited resolution scenarios. First however, we need to introduce these model. 


\section{Sub-grid scale models}

Despite the staggering range of SGS models available for incompressible hydrodynamics, only a comparative few have been transferred to the compressible regime and even fewer are extended to magnetised plasmas (compressible or otherwise). This chapter presents some of the SGS models available for the SGS stress and electromotive force. They can be separated into two groups: functional and structural. The differentiation is mostly heuristic and is rooted in the philosophy of the modelling approach. Functional models focus on a key effect that the SGS terms have on the resolved scales, e.g. energy transfer, and attempt to reproduce it disregarding the structure (geometric, dynamic, statistical, etc.) of the SGS terms themselves. Structural models, on the other hand, develop from a set of key properties of the SGS terms, e.g. the orientation of the proper axes of the SGS stress tensor, the probability distribution function, et cetera. In that sense a detailed knowledge of the properties of the SGS terms is required in order to develop such closures. The idea is that the effect which the SGS dynamics has on the resolved scale would naturally develop from these properties.

Alternatively, this dichotomy can be viewed from the perspective of the deconvolution problem. As we already discussed the finite resolution effects present in the shape of a convolution operator. In principle we could simply try to invert that operator to obtain the full fluid description/solution - this is the deconvolution approach to LES. However, we don't have the entire information in the simulated fields, there is a cut-off at the Nyquist frequency. Thus the deconvolution problem over the entire spectral domain of validity of fluid dynamics is ill-posed. It can be however be separated [43] in soft and hard deconvolution problems.

The hard deconvolution problem is to recover the information below the Nyquist frequency of a simulation and include its effects on the resolved scales - this is the ill-posed part. No information about that is contained within the simulation - it has to come from the model itself, e.g. via mimicking microscopic processes, regularization, etc. [43]. This falls within the domain of functional models. Usually a universality hypothesis is involved: at sufficiently small scales the turbulence is homogeneous and isotropic enough that the particulars of the integral scales are unimportant, e.g the geometry (anisotropy) of the large-scale flow. Sometimes they might involve direct models for e.g. $\nabla \cdot \tau$ and $\nabla \times \mathcal{E}$ as this is how the SGS terms enter the resolved equations motion. The associated soft deconvolution problem deals with the domain of scales represented on the grid but not resolved, e.g. contaminated by strong truncation errors, aliasing, etc. Here, in principle the information is available, but garbled beyond recognition. The soft deconvolution problem is to decode it. Structural models are most suitable to tackle this. The main assumption is that the dominant error comes from the commutator of the filtering operator with multiplication, i.e. from the misrepresentation of the nonlinear terms on the grid. 
Yet another way to look at this is that the hard deconvolution problem deals with the finite resolution effects for a single field, while the soft deconvolution problem deals with the information lost in the commutator of the finite resolution operator with multiplication.

We make this more concrete in the following. We begin with the derivation and development of a new structural model for compressible MHD. Then we proceed with a short review of other available models (one structural and several functional), popular in the literature. We finish with a few words on stability, coefficient determination and the possibility of model mixing.

\subsection{Non-linear model}

Like most of LES the presented model originates from incompressible hydrodynamics. It has links to the gradient diffusion model (pioneered by $[22,54]$ ) but it is build on a different foundation. The original gradient diffusion models are constructed by Taylor expansion of an SGS term with respect to the turbulent fluctuation or the entire (unfiltered) fields, (e.g. $\mathbf{u}^{\prime}$ or $\mathbf{u}$ for $\tau^{\mathrm{u}}$ ). Since these quantities contain rapidly fluctuating components at the filter scale their Taylor expansion is fundamentally questionable. In contrast, the proposed model is based on the expansion of the filtering kernel itself.

In the following we present a derivation of the model, followed by related arguments which lead to the same or similar models. We conclude with variations of the model based on regularization arguments.

\subsubsection{Yeo-Bedford series expansion}

The model is an application of the Yeo-Bedford (YB) series expansions [103, 104]. The expansion was originally developed for hydrodynamic applications and was recently (only once to our knowledge) applied to the EMF in incompressible MHD by [6, 31]. More recently the procedure has been used to model the transport of a passive scalar [5].

The method begins by constructing the gradient expansion of the filtering operator and its complement (i.e. the corresponding high-pass filter). For the classical filters this can be done by moving to Fourier space ${ }^{1}$. Since the filtering operator is a convolution, in Fourier space it simply transforms into a multiplication, i.e.

$$
\widehat{G * f}=\widehat{G} \widehat{f}
$$

The quantity $\widehat{G}$ is known as the filter's transfer function. Trivially, $1 / \widehat{G}$ is the transfer function of the high-pass complement filter $G^{-1}$. Performing a Taylor expansion in Fourier space and transforming back to physical space gives the required result. For

$$
{ }^{1} \widehat{f}(\mathbf{k})=\int_{-\infty}^{\infty} f(x) \exp (-i \mathbf{k} \cdot \mathbf{x}) d^{3} \mathbf{x}
$$


example for the Gaussian filter we have

$$
\begin{gathered}
\widehat{G}=\exp \left(-\frac{\Delta^{2}}{24} \mathbf{k}^{2}\right)=\sum_{n=0}^{\infty} \frac{(-1)^{n}}{n !}\left(\frac{\Delta^{2}}{24} \mathbf{k}^{2}\right)^{n} \Rightarrow G=\sum_{n=0}^{\infty} \frac{1}{n !}\left(\frac{\Delta^{2}}{24} \nabla^{2}\right)^{n}, \\
\frac{1}{\widehat{G}}=\exp \left(\frac{\Delta^{2}}{24} \mathbf{k}^{2}\right)=\sum_{n=0}^{\infty} \frac{1}{n !}\left(\frac{\Delta^{2}}{24} \mathbf{k}^{2}\right)^{n} \Rightarrow G^{-1}=\sum_{n=0}^{\infty} \frac{(-1)^{n}}{n !}\left(\frac{\Delta^{2}}{24} \nabla^{2}\right)^{n} .
\end{gathered}
$$

Note that with this choice of normalization of the Gaussian filter this expansion up to second order is also valid for the box filter. Now we have for a generic field $f$ :

$$
\begin{array}{r}
\bar{f}=G \star f=\sum_{n=0}^{\infty} \frac{1}{n !}\left(\frac{\Delta^{2}}{24} \nabla^{2}\right)^{n} f, \\
f=G^{-1} \star \bar{f}=\sum_{n=0}^{\infty} \frac{(-1)^{n}}{n !}\left(\frac{\Delta^{2}}{24} \nabla^{2}\right)^{n} \bar{f} .
\end{array}
$$

Applying eq. (2.3) to a term of the form $\bar{h}=\overline{f g}$ gives a series in terms of $(f g)$. Applying eq. (2.4) to $f$ and $g$ in each term of this series gives an expansion of $\bar{h}$ in terms of $\bar{f}$ and $\bar{g}$. Finally, collecting terms by filter scale (or equivalently by differential order), and letting $a=\Delta^{2} /(24)$ gives the result

$$
\overline{f g}=\bar{f} \bar{g}+2 a \bar{f}_{, k} \bar{g}_{, k}+\frac{1}{2 !}(2 a)^{2} \bar{f}_{, k l} \bar{g}_{, k l}+\frac{1}{3 !}(2 a)^{3} \bar{f}_{, k l m} \bar{g}_{, k l m}+O\left(a^{4} \nabla^{8}\right) .
$$

Note that the coefficients in the expansion are related to moments of the transfer function. This relationship is based on the orthogonality of the sine and cosine functions in the Fourier expansion and thus holds for a general filter kernel. In particular the second coefficient $2 a$ is given by the second moment of the transfer function. Furthermore, [14] have derived a closed form expression for the coefficients in eq. (2.5) for a symmetric filter with infinitely differentiable transfer function. It is given as the Taylor coefficients in the expansion generating function $F(f, g)=G(-i(f+g)) /(G(-i f) G(-i g))$. It is clear that for a symmetric filter only the even coefficients are non-zero (since it has a real transfer function). This imposes a symmetry with respect to the differentiated co-ordinate/index, which can be used to easily derive higher order terms in the series namely each co-ordinate appears exactly twice and each field is differentiated at most once with respect to a co-ordinate.

Naturally the expansion can be extended to an SGS term of any order, specifically $\tau^{\mathrm{u}}$ which in the compressible case contains three fields.

$$
\begin{aligned}
\overline{f g h}= & \bar{f} \bar{g} \bar{h}+2 a\left(\bar{f}_{, k} \bar{g}_{, k} \bar{h}+\bar{f}_{, k} \bar{g} \bar{h}_{, k}+\bar{f}_{\bar{g}, k} \bar{h}_{, k}\right)+ \\
& \frac{1}{2 !}(2 a)^{2}\left(\bar{f}_{, k l} \bar{g}_{, k l} \bar{h}+\bar{f}_{, k l} \bar{g} \bar{h}_{, k l}+\bar{f}_{\bar{g}_{, k l}} \bar{h}_{, k l}+2 \bar{f}_{, k} \bar{g}_{, k l} \bar{h}_{, l}+2 \bar{f}_{, k} \bar{g}_{, l} \bar{h}_{, k l}+2 \bar{f}_{, k l} \bar{g}_{, k} \bar{h}_{, l}\right)+ \\
& +O\left(a^{3} \nabla^{6}\right),
\end{aligned}
$$

To first order this technique leads to the following results for the primary SGS terms 


$$
\begin{aligned}
\overline{\rho u_{i} u_{j}}-\bar{\rho} \bar{u}_{i} \bar{u}_{j} & =2 a \bar{\rho} \bar{u}_{i, k} \bar{u}_{j, k}+2 a \bar{\rho}_{, k}\left(\bar{u}_{i, k} \bar{u}_{j}+\bar{u}_{i} \bar{u}_{j, k}\right), \\
\overline{B_{i} B_{j}}-\bar{B}_{i} \bar{B}_{j} & =2 a \bar{B}_{i, k} \bar{B}_{j, k}, \\
(\overline{\mathbf{u} \times \mathbf{B}}-\overline{\mathbf{u}} \times \overline{\mathbf{B}})_{i} & =2 a \epsilon_{i j k} \bar{u}_{j, l} \bar{B}_{k, l} .
\end{aligned}
$$

As can be seen the expansion is in terms of the simply filtered fields and no account is taken of the Favre filter. This is problematic in compressible finite volume codes which evolve the momentum instead of the velocity field. Indeed, the Favre-filtering operator in spectral space involves a convolution with the full (unresolved) density field which renders the procedure (specifically the high-pass filter inversion) considerably more involved. For simulations which evolve the velocity field, however this is a valid alternative, as has been shown for compressible HD in e.g. [10].

Before we deal with the issue of compressibility however, a note on convergence is required. The method rests on infinite series expansion and interchanging the order of summation/limiting operations. (e.g. when converting forward and backward through Fourier space). This is justified only if the series in question are absolutely convergent. In [71] a set of conditions is derived for the forward expansion eq. (2.3) to hold. They find that symmetry of the kernel and non-negativity of its transfer function are sufficient conditions for convergence. Additionally, they present a qualitative criterion that convergence rate decreases as the dissipative strength of the filter increases. Since the backward expansion eq. (2.4) differs from the forward one only in an alternating sign both results hold for it as well. Additionally, since the final closures employ expansion in terms of filtered fields, only the filtered fields need to satisfy a smoothness criterion - namely to be $n$-times differentiable for the $2 n$-order closure. For the second order closure this is ensured by the filtering itself.

\subsubsection{Compressible extension via recurrence relation}

We address the compressibility issue raised above by looking for an expression for $\overline{\mathbf{u}}$ as a function of $\tilde{\mathbf{u}}$. This can be substituted in e.g. eq. (2.7) to obtain a Favre-friendly version $^{2}$.

Here we employ a recurrence technique. By definition the Favre-weighted velocity is given by $\tilde{\mathbf{u}}=\overline{\mathbf{u} \rho} / \bar{\rho}$. It can be expanded as the series

$$
\tilde{u}_{i}=\bar{u}_{i}+2 a y_{, k} \bar{u}_{i, k}+2 a^{2}\left(y_{, k l}+y_{, k} y_{, l}\right) \bar{u}_{i, k l}+O\left(a^{3}\right),
$$

where we define for brevity $y=\ln \bar{\rho}$. If eq. (2.10) represents an absolutely convergent series, it can be rearranged to

$$
\bar{u}_{i}=\tilde{u}_{i}-2 a y_{, k} \bar{u}_{i, k}-2 a^{2}\left(y_{, k l}+y_{, k} y_{, l}\right) \bar{u}_{i, k l}-O\left(a^{3}\right) .
$$

This can then be substituted into itself. The first recursion gives

$$
\bar{u}_{i}=\tilde{u}_{i}-2 a y_{, k} \tilde{u}_{i, k}+2 a^{2}\left(2 y_{, k}\left(y_{, l} \bar{u}_{i, l}\right)_{, k}-\left(y_{, k l}+y_{, k} y_{, l}\right) \tilde{u}_{i, k l}\right)-O\left(a^{3}\right) .
$$

\footnotetext{
${ }^{2}$ In this approach care should be taken with the definition of the EMF - whether it is given in terms of $\overline{\mathbf{u}}$ or $\tilde{\mathbf{u}}$.
} 
Substituting eq. (2.11) again gives

$$
\bar{u}_{i}=\tilde{u}_{i}-2 a y_{, k} \tilde{u}_{i, k}-2 a^{2}\left(\left(y_{, k l}-y_{, k} y_{, l}\right) \tilde{u}_{i, k l}-2 y_{, k} y_{, k l} \tilde{u}_{i, l}\right)-O\left(a^{3}\right) .
$$

Now, the definition of $\tau^{\mathrm{u}}$ can be restated as

$$
\tau_{i j}^{\mathrm{u}}=\overline{\rho u_{i} u_{j}}-\frac{\overline{\rho u_{i}} \overline{\rho u_{j}}}{\bar{\rho}} .
$$

Using the incompressible YB formulation on each of the terms to second order, this can be rearranged into

$$
\begin{aligned}
\tau_{i j}^{\mathrm{u}}= & 2 a \bar{\rho} \bar{u}_{i, k} \bar{u}_{j, k}+ \\
& 2 a^{2} \bar{\rho}\left(\bar{u}_{i, k l} \bar{u}_{j, k l}+2 y_{, k}\left(\bar{u}_{i, k l} \bar{u}_{j, l}+\bar{u}_{i, l} \bar{u}_{j, k l}\right)-\right. \\
& \left.2 y_{, k} y_{, l} \bar{u}_{i, k} \bar{u}_{j, l}+2\left(y_{, k l}+y_{, k} y_{, l}\right) \bar{u}_{i, k} \bar{u}_{j, l}\right) \\
& +O\left(a^{3}\right) .
\end{aligned}
$$

Substituting eq. (2.13) and simplifying we obtain

$$
\tau_{i j}^{\mathrm{u}}=2 a \bar{\rho} \tilde{u}_{i, k} \tilde{u}_{j, k}+2 a^{2} \bar{\rho}\left(\tilde{u}_{i, k l} \tilde{u}_{j, k l}-2 y_{, k l} \tilde{u}_{i, k} \tilde{u}_{j, l}\right)+O\left(a^{3}\right) .
$$

Alternatively, the same expression can be reached by substituting the recurrence relation eq. (2.11) directly into eq. (2.7).

We see that up to first order the compressibility effects are taken into account by the mass-weighted filtering itself. The density derivatives appear as second order corrections. This is due to the symmetry of the stress tensor $\left(\tau_{i j}=\tau_{j i}\right)$. Crucially, it is the logarithm of the density and not the density itself that plays the central role in the Favre-corrections. This implies that even for relatively strong density gradients the trivial map $\bar{\square} \rightarrow \tilde{\square}$ may be a sufficient approximation. This approximation has been tested with encouraging results in [48].

Applying the same method to the EMF, we obtain the following expression

$$
\begin{aligned}
\mathcal{E}_{i}= & 2 a \epsilon_{i j k}\left(\tilde{u}_{j, l} \bar{B}_{k, l}-y_{, l} \tilde{u}_{j, l} \bar{B}_{k}\right)+ \\
& 2 a^{2} \epsilon_{i j k}\left\{\tilde{u}_{j, l m} \bar{B}_{k, l m}-2\left(y_{, l m} \tilde{u}_{j, l}+y_{, l} \tilde{u}_{j, l m}\right) \bar{B}_{k, l}+\right. \\
& \left.\left(2 y_{, l} y_{, l m} \tilde{u}_{j, m}+\left(y_{, p} y_{, l}-y_{, p l}\right) \tilde{u}_{j, p l}\right) \bar{B}_{k}\right\}+O\left(a^{3}\right) .
\end{aligned}
$$

Here, due to the asymmetry between $\tilde{u}$ and $\bar{B}$ the Favre corrections appear already at first order in the filter scale. In addition, the second order corrections are more extensive than in the case of $\tau^{\mathrm{u}}$.

Equations (2.16) and (2.17) are the main result of this section. They form the core of the newly proposed SGS model for compressible MHD, along with eq. (2.8), which is formally insensitive to compressibility effects. 


\subsubsection{Link with ancestor and sibling models}

It should be noted that in hydrodynamics expressions similar to eq. (2.5) have been reached by alternative methods

The earliest closures for LES based on a Taylor series expansion is the tensor-diffusivity model and can be dated back to $[22,54]$. They perform Taylor expansions separately on the Leonard, cross and Reynolds SGS terms of incompressible HD. Crucially they expand the turbulent fluctuations, which are not smoothly varying over a filter scale. This is why the approach had been called 'mathematically indefensible' in [56]. An alternative derivation and compressible extension is derived in [98]. They obtain eq. (2.3) by Taylor expansion of the full field variable under the filtering operator. The series is then truncated to second order to obtain $\bar{\rho}=\rho+O\left(\Delta^{2}\right)$ and $\tilde{\mathbf{u}}=\mathbf{u}+O\left(\Delta^{2}\right)$. Compressibility is incorporated by inserting the additional expansion $\bar{\rho}^{-1}=\left(\rho+O\left(\Delta^{2}\right)\right)^{-1}=\rho^{-1}+O\left(\Delta^{2}\right)$ into the definition of the Favre-filtered velocity. These expressions are inverted and substituted in eq. (2.3) applied to $\tau$. The result is identical to eq. (2.16) up to second order. However, the basis is still the Taylor expansion of fields which are not necessarily smooth over a filter length. They propose to put the model on more solid footing by applying it to the scale similarity closure (will be detailed in section 2.2.1). In that case the expansion will be in terms of filtered quantities only. The consequence is that the residual term, still of second order, takes a different form and now represents additionally the error induced by the scale-similarity model. We are not aware of applications of this procedure to MHD.

An alternative derivation is provided by the approximate deconvolution method. It is again based on the truncation of an infinite series depicting the inverse of the filtering operator. Originally designed for image processing [95], this method has been applied to LES by [85] for a wide variety of flows [85-90]. Considering a filter $G$, an approximation of its inverse $G^{-1}$ is formally given by

$$
G^{-1} \approx Q=\sum_{n=0}^{N}(\mathrm{I}-G)^{n},
$$

with I, the identity operator. The sum does not necessarily converge as $N \rightarrow \infty$. However, the error can be optimised by taking a fixed $N$. It is claimed that $N=3$ gives 'good results' for compressible HD and going beyond $N=5$ is unnecessary [43]. The results of this approach applied to a Gaussian filter with $N=2$ are equivalent with the direct Taylor series expansion above up to second order $\left(O\left(\Delta^{4}\right)\right)$ [43]. The different motivation/derivation path is again revealed at higher orders.

\subsubsection{Model renormalization/regularization}

Here we describe several model variations, based on regularization and a posteriori arguments. They have been summarised at the end of the section in tab. 2.1

Firstly, the shape of the filter kernel associated with an actual LES does not correspond either to a Gaussian or to a box kernel. It is a combination of the filtering induced by multiple source, e.g. the grid spacing, the discretization of the time and space 
differential operators, flux limiting, et cetera. Recall that the coefficient $a$ is related to the second moment of the filter kernel. Thus in the absence of the exact LES kernel for a particular numerical scheme, or alternatively to provide a general starting point for a generic code, we insert a free coefficient $C_{\square}$ in the model. The value of this coefficient and its variation encode the error induced both by the functional form of the model and the assumed analytic kernel. To test the effect of the compressibility terms we shall further allow for two independent coefficients for the two components of the EMF.

Secondly, it has been widely recognised in hydrodynamics (e.g. [9, 22, 29, 43, 74]) that structural models suffer from insufficient SGS dissipation. This can be understood in two complementary ways. On the one hand, the truncation of the series expansion is equivalent to omitting information about the very small (grid) scales where dissipation ultimately takes place within the cascade hypothesis. On the other hand, consider the limitations imposed by the Nyquist frequency. Fields resolved on a given grid can carry information only up to the corresponding wave number. Thus any closure based on inverting the filtering/resolution operator cannot reproduce information for larger wave numbers. Recall that this is the so-called hard deconvolution problem [43], which cannot be properly addressed in a priori tests. In both cases the omitted scales are expected to be strongly dominated by dissipation effects.

Additionally, structural models can become unstable, e.g. [45]. The issue is that since the low-pass filter suppresses the small scales, its inverse respectively enhances them. Since the smallest scales give rise to the largest gradients, they may dominate a gradient-based model. In numerical simulations the small (near-grid) scales suffer from the worst round-off errors. The combination of the two effects can lead to artificial instabilities $^{3}$. For a quantification of the effect see e.g. [98].

The model strength can be locally calibrated by renormalizing eqs. (2.8) and (2.16) by the kinetic and magnetic SGS energies respectively. This form of renormalization has been applied in compressible hydrodynamics, e.g. by [77, 102], with great success. Since the turbulent energies constitute the traces of $\tau^{\mathrm{u}}$ and $\tau^{\mathrm{b}}$ already, it makes sense to use for the model only the deviatoric parts of eqs. (2.8) and (2.16). In that case, expressions for the SGS energies need to be additionally provided. Several choices for this will be discussed in section 2.3. Depending on the form of these expressions it maybe advantageous to use the full renormalized model as well. The EMF is not renormalized in this manner at this stage. However, a suitable field could be (a function of) the turbulent cross-helicity.

\footnotetext{
${ }^{3}$ Even though this is somewhat remedied by the truncation of the series, it is not fully resolved.
} 


\begin{tabular}{c|c} 
Proposed Model variations & $\tau_{i j}^{\mathrm{u}}$ \\
\hline \hline basic & $2 C_{\mathrm{u}} a \bar{\rho} \tilde{u}_{i, k} \tilde{u}_{j, k}$ \\
\hline split & $2 C_{\mathrm{u} 1} a \bar{\rho}\left(\tilde{u}_{i, k} \tilde{u}_{j, k}\right)^{*}+\frac{2}{3} C_{\mathrm{u} 2} a \bar{\rho} \tilde{u}_{m, l} \tilde{u}_{m, l} \delta_{i j}$ \\
\hline renormalized & $2 C_{\mathrm{u}} a E_{\mathrm{sgs}}^{\mathrm{u}}\left(\frac{\tilde{u}_{i, k} \tilde{u}_{j, k}}{\tilde{u}_{m, l} \tilde{u}_{m, l}}-\frac{1}{3} \delta_{i j}\right)+E_{\mathrm{sgs}}^{\mathrm{u}}$ \\
\hline \hline
\end{tabular}

\begin{tabular}{c|c} 
& $\tau_{i j}^{\mathrm{b}}$ \\
\hline \hline basic & $2 C_{\mathrm{b}} a \bar{B}_{i, k} \bar{B}_{j, k}$ \\
\hline split & $2 C_{\mathrm{b} 1} a\left(\bar{B}_{i, k} \bar{B}_{j, k}\right)^{*}+\frac{2}{3} C_{\mathrm{b} 2} a \bar{B}_{m, l} \bar{B}_{m, l} \delta_{i j}$ \\
\hline renormalized & $2 C_{\mathrm{b}} a E_{\mathrm{sgs}}^{\mathrm{b}}\left(\frac{\bar{B}_{i, k} \bar{B}_{j, k}}{\bar{B}_{m, l} \bar{B}_{m, l}}-\frac{1}{3} \delta_{i j}\right)+E_{\mathrm{sgs}}^{\mathrm{b}}$ \\
\hline \hline
\end{tabular}

\begin{tabular}{c|c}
\hline & $\mathcal{E}_{i}$ \\
\hline \hline basic & $2 C_{\mathcal{E}} a \epsilon_{i j k}\left(\tilde{u}_{j, l} \bar{B}_{k, l}-y_{, l} \tilde{u}_{j, l} \bar{B}_{k}\right)$ \\
\hline split & $2 C_{\mathcal{E} 1} a \epsilon_{i j k} \tilde{u}_{j, l} \bar{B}_{k, l}-2 C_{\mathcal{E} 2} a \epsilon_{i j k} y_{, l} \tilde{u}_{j, l} \bar{B}_{k}$ \\
\hline \hline
\end{tabular}

Table 2.1: Proposed model variations for the SGS terms: the basic 1-coefficient compressible closure, the split 2-coefficient version and the renormalized SGS stress

\subsection{Alternative models}

\subsubsection{Scale-similarity model}

The main established structural model for MHD is the scale-similarity model. It originates in incompressible hydrodynamics [9] where it is based on the properties of the inertial range. Namely, assuming that the energy transfer across scales is self-similar in the inertial range and that the resolution limit falls within that range, the smallest 
resolved scales should have the same structure as the largest unresolved scales. The argument is finished by assuming that the largest unresolved scales dominate the total unresolved flow (which is reasonable, e.g. for the energy spectrum). For fields $f$ and $g$ this can be expressed via the approximations

$$
\begin{aligned}
f^{\prime} & \approx \overline{f^{\prime}}=\bar{f}-\overline{\bar{f}}, \\
\overline{f^{\prime} \bar{g}} & \approx \overline{f^{\prime}} \overline{\bar{g}} .
\end{aligned}
$$

Recall that $f^{\prime}$ denotes the turbulent fluctuations of a field $f$, i.e. $f^{\prime}=f-\bar{f}$. Thus the first equation posits that the filtered fluctuations are representative of the total fluctuations. Similarly, the second equations approximates the interaction between fluctuations and filtered fields by the interaction between the corresponding filtered quantities. Combining the two equations leads to a model for the quadratic central moment

$$
\mathcal{C}(f, g)=\overline{\bar{f}} \bar{g}-\overline{\bar{f}} \overline{\bar{g}}
$$

A key issue here is the meaning of the second filtering operation. Originally, in $a$ priori tests it is taken to be simply a repetition of the original filter ${ }^{4}$. This has been developed and investigated by [55] and applied to compressible MHD turbulence in $[16,18]$. However, drawing upon the original model assumptions and in view of $a$ posteriori applications, it has been suggested [15] to use a test filter for the second filtering operation. It typically has the same kernel as the original filter (achieved in LES by e.g applying a coarser grid) and double the filter scale, i.e. $2 \Delta$. This is the interpretation of the double filtering which will be used here. The model reads ${ }^{5}$

$$
\begin{aligned}
& \tau_{i j}^{\mathrm{u}}=\bar{\rho}\left(\widetilde{\tilde{u}_{i} \tilde{u}_{j}}-\widetilde{\tilde{u}}_{i} \widetilde{\tilde{u}}_{j}\right) \\
& \tau_{i j}^{\mathrm{b}}=\left(\overline{\bar{B}}_{i} \bar{B}_{j}-\overline{\bar{B}}_{i} \overline{\bar{B}}_{j}\right) \\
& \mathcal{E}=\overline{\tilde{\mathbf{u}} \times \overline{\mathbf{B}}}-\tilde{\widetilde{\mathbf{u}}} \times \overline{\overline{\mathbf{B}}} .
\end{aligned}
$$

It has been recorded that in hydrodynamics $[9,55]$ and MHD [16] the scale-similarity model under-predicts the SGS energy dissipation rate and can even be numerically unstable. This can be easily understood analytically. The model essentially handles the SGS Leonard while neglecting the cross and Reynolds term. Since, it only contains the top end of the unresolved scales it represents little of the crucial dynamics of the interface between the turbulent and dissipation scales, the range which controls the ultimate energy dissipation. To combat this mixed models, linear combinations with other strictly dissipative models, have been proposed e.g. [9, 55].

\footnotetext{
${ }^{4}$ Note that this is meaningful only for filters which are not Reynolds operators.

${ }^{5}$ Note that the model can be incorporated into the approximate deconvolution family of 2.18 with $N=0[43$, p. 99].
} 


\subsubsection{Eddy-dissipation and dynamo models}

An alternative approach to modelling (and the original idea in LES) is given by the so-called functional method. Here we begin with an idea of the physical effects of the SGS scales on the resolved flow and then try to incorporate that effect into the LES equations. The correlation between the model and SGS terms is immaterial as long as the effect on the large scales is reproduced. In [55] a set of necessary conditions has been derived (for HD) under which a model displays the correct LES statistics irrespective of its internal structure. There is large body of work in incompressible (e.g. [74]) and compressible (e.g. [43, 76]) HD. More recently incompressible (e.g. $[4,61,62,106,109,117])$ and compressible (e.g. [18, 59]) MHD closures have also been investigated. Here we present a choice of recently used functional models (based on $[1,16,18,59,61,109])$ for comparison with the structural ones above.

\section{Eddy-viscosity}

The eddy-viscosity assumption originates in hydrodynamics and is concerned with arguably the most important effect of turbulence on the resolved scales - the energy cascade. We recall that the transfer of energy between resolved and unresolved scales ( $E_{\mathrm{res}}^{\mathrm{u}} \leftrightarrow E_{\mathrm{sgs}}^{\mathrm{u}}$ ) (barring diffusion) in HD is given by $\epsilon=-\tau_{i j} \tilde{\mathcal{S}}_{i j}$. This implies that the dissipative properties of the hydrodynamic SGS stress $\tau$ are encoded in its components aligned with the eigenvectors of $\tilde{\mathcal{S}}$ (more precisely, since $\tilde{\mathcal{S}}$ is symmetric its eigenvectors form and orthogonal basis, thus the dissipation is given by the diagonal components of $\tau$ in that basis). The simplest model for $\tau$ then would be

$$
\tau=-2 \bar{\rho} \nu_{\mathrm{sgs}} \tilde{\mathcal{S}}
$$

for some scalar field $\nu_{\text {sgs }}$. In other words a homogeneous and isotropic scaling of the effect of $\tilde{\mathcal{S}}$ ). Note that this is also the form of the microphysical viscous stress for a Newtonian fluid, $\bar{\rho} \sigma=-2 \bar{\rho} \nu \tilde{\mathcal{S}}^{*}$. Thus, the model claims that the only effect of unresolved turbulence is to locally (since $\nu_{\mathrm{sgs}}$ can be spatially varying) boost the microscopic viscosity, i.e. decrease the effective Reynolds number. The scalar field $\nu_{\text {sgs }}$ is the turbulent or eddy-viscosity. It can incorporate flow characteristics like, for example, the turbulence intensity. This class of models, accordingly named eddy-viscosity models $(\mathrm{EV})$ is probably classically the most widely used one. It has had big success in HD and engineering applications.

The addition of magnetic fields can be incorporated into this paradigm as well. The SGS stress is redefined to include $\tau^{\mathrm{b}}$. This doesn't change the structure of the resolved kinetic energy dissipation term (still $\tau \cdot \tilde{\mathcal{S}}$ ). Thus the MHD SGS stress closure retains the form of eq. (2.21) but it may have modified form of $\nu_{\text {sgs. }}$. The resolved electromotive force now also enters through the magnetic energy dissipation via the term $\mathcal{E} \cdot \overline{\mathbf{J}}$. The same logic leads to the eddy-resistivity closure (ER) with eddy- (or turbulent, or anomalous) resistivity $\eta$,

$$
\mathcal{E}=-\eta_{\mathrm{sgs}} \overline{\mathbf{J}}
$$


Dimensionally, or using Prandtl's mixing length theory $[52,68,69], \nu_{\mathrm{sgs}}$ and $\eta_{\mathrm{sgs}}$ can be expressed by a length scale (presumably the SGS length scale $\Delta$ ) and a time scale (e.g some dissipation rate $\epsilon$ ), for example $\nu \sim \Delta^{4 / 3} \epsilon^{1 / 3}$.

A plethora of models comes out of the way in which the dissipation rates are chosen and approximated. The simplest model is to take them spatially constant:

$$
\nu_{\mathrm{sgs}}=C_{1} \Delta^{-4 / 3}, \quad \eta_{\mathrm{sgs}}=D_{1} \Delta^{-4 / 3} .
$$

If we continue the EV/ER reasoning and link $\nu_{\text {sgs }}$ and $\eta_{\text {sgs }}$ to the dissipation rates of the (resolved) kinetic and magnetic energies respectively, this is revealed as the MHD generalization of the Kolmogorov model [101]. Physically, the reasoning for the constant dissipation rates is that the spatial variation is negligible, if the non-linear interaction between SGS kinetic and magnetic energy is much smaller than their dissipation rates, as argued by [1]. Note, that here the coefficients $C_{1}$ and $D_{1}$ are not unitless.

Alternatively, continuing with the dimensional/mixing length approach, the dissipation rate of a quantity $f$ can be given by $\epsilon_{f} \sim f / t_{f} \sim f v_{f} / l_{f}$ for some characteristic time scale $t_{f}$, length scale $l_{f}$ and velocity scale $v_{f}$. For example for the SGS kinetic energy this can be simplified to $\epsilon_{E_{\mathrm{sgs}}^{\mathrm{u}}} \sim\left(E_{\mathrm{sgs}}^{\mathrm{u}}\right)^{3 / 2} \Delta^{1 / 3}$. This approach is tied to the cascade property of turbulence, i.e. the existence of inertial range in the spectrum of $f$ implies transfer which is primarily local in spectral space. So we shall refer to it as the cascade approximation.

We still need to choose characteristic quantities and we can again use the EV/ER heuristics to select the SGS kinetic and magnetic energies as calibrators for the local strength of the transfer. This leads to the expressions

$$
\begin{aligned}
& \nu_{\mathrm{sgs}}=C_{1} \Delta \sqrt{E_{\mathrm{sgs}}^{\mathrm{u}} / \bar{\rho}}, \\
& \eta_{\mathrm{sgs}}=D_{1} \Delta \sqrt{E_{\mathrm{sgs}}^{\mathrm{b}}} .
\end{aligned}
$$

Notice that both of these models eqs. (2.23) and (2.24) are strictly dissipative (the eddy viscosity and resistivity are positive definite) - we shall come back to this in section 2.3.4. However, the existence of an inverse cascade (backscatter) of energy (and cross-helicity and magnetic helicity) has been well documented (e.g. since [67] and more recently in [63]). Even in HD it can comprise about $10-20 \%$ of the total energy transfer [79]. This can be one of the reasons why these models have been found to be too dissipative [1]. To incorporate the backscatter [61] propose a model for incompressible MHD based on the cross-helicity dissipation. Similarly to the energy case they link eddy viscosity with the kinetic contribution and the eddy resistivity with the magnetic one, so that the respective dissipation rates are expressed as $\epsilon_{H_{\text {res }}}^{\mathrm{u}} \sim \tilde{\mathcal{S}}_{i j} \overline{\mathcal{M}}_{i j}$ and $\epsilon_{H_{\text {res }}}^{\mathrm{b}} \sim \overline{\mathbf{J}} \cdot \tilde{\Omega}$. This leads to

$$
\begin{aligned}
& \nu_{\mathrm{sgs}}=C_{1} \Delta^{2} \sqrt{\left|\tilde{\mathcal{S}}_{i j} \overline{\mathcal{M}}_{i j}\right|} \\
& \eta_{\mathrm{sgs}}=D_{1} \Delta^{2} \operatorname{sgn}(\overline{\mathbf{J}} \cdot \tilde{\boldsymbol{\Omega}}) \sqrt{\overline{\mathbf{J}} \cdot \tilde{\boldsymbol{\Omega}}}
\end{aligned}
$$

They argue that this is a better gauge of the turbulent velocity and magnetic fields since it captures robustly the transfer between kinetic and magnetic energy. Only $\eta_{\mathrm{sgs}}$ 


\begin{tabular}{c|c|c} 
EV $/$ ER Models & $\tau^{*}=-2 \nu_{\mathrm{sgs}} \bar{\rho} \tilde{\mathcal{S}}^{*}$ & $\mathcal{E}=-\eta_{\mathrm{sgs}} \overline{\mathbf{J}}$ \\
\hline \hline Constant & $\nu_{\mathrm{sgs}}=C_{1} \Delta^{4 / 3}$ & $\eta_{\mathrm{sgs}}=D_{1} \Delta^{4 / 3}$ \\
\hline Energy & $\nu_{\mathrm{sgs}}=C_{1} \Delta \sqrt{E_{\mathrm{sgs}}^{\mathrm{u}} / \bar{\rho}}$ & $\eta_{\mathrm{sgs}}=D_{1} \sqrt{E_{\mathrm{sgs}}^{\mathrm{b}} / \bar{\rho}}$ \\
\hline Cross-helicity & $\nu_{\mathrm{sgs}}=C_{1} \Delta^{2} \bar{\rho}^{-1 / 4} \sqrt{\left|\widetilde{S}^{*} \cdot \bar{M}\right|}$ & $\eta_{\mathrm{sgs}}=D \Delta^{2} \bar{\rho}^{-1 / 4} \operatorname{sgn}(\bar{J} \tilde{\Omega}) \sqrt{|\bar{J} \cdot \tilde{\Omega}|}$ \\
\hline Cross-helicity2 & $\nu_{\mathrm{sgs}}=C_{1} \Delta^{2} \bar{\rho}^{-1 / 4} \sqrt{\left|H_{\mathrm{sgs}}\right|}$ & $\eta_{\mathrm{sgs}}=D \Delta^{2} \bar{\rho}^{-1 / 4} \operatorname{sgn}\left(H_{\mathrm{sgs}}\right) \sqrt{\left|H_{\mathrm{sgs}}\right|}$ \\
\hline
\end{tabular}

Table 2.2: Turbulent/eddy viscosity/resistivity models.

is allowed to change sign but they postulate that this is sufficient due to the strong exchange of resolved kinetic and magnetic energy and the existence of dominant inverse magnetic helicity cascade. In this model there is no direct kinetic energy backscatter, rather resolved kinetic energy is replenished by the small scales via the channel $E_{\mathrm{res}}^{\mathrm{u}} \rightarrow$ $E_{\mathrm{sgs}}^{\mathrm{u}} \leftrightarrow E_{\mathrm{sgs}}^{\mathrm{b}} \leftrightarrow E_{\mathrm{res}}^{\mathrm{b}} \leftrightarrow E_{\mathrm{res}}^{\mathrm{u}}$. The model has been applied directly to a compressible environment in $[16,18]$ by the map $\nu_{\mathrm{sgs}} \rightarrow \bar{\rho} \nu_{\mathrm{sgs}}, \eta_{\mathrm{sgs}} \rightarrow \eta_{\mathrm{sgs}}$. Since in this case the coefficients become dimensional, we recast it to

$$
\begin{aligned}
& \nu_{\mathrm{sgs}}=C_{1} \Delta^{2} \bar{\rho}^{-1 / 4} \sqrt{\left|\tilde{\mathcal{S}}_{i j} \overline{\mathcal{M}}_{i j}\right|} \\
& \eta_{\mathrm{sgs}}=D_{1} \Delta^{2} \bar{\rho}^{-1 / 4} \operatorname{sgn}(\overline{\mathbf{J}} \cdot \tilde{\boldsymbol{\Omega}}) \sqrt{|\overline{\mathbf{J}} \cdot \tilde{\boldsymbol{\Omega}}|} .
\end{aligned}
$$

We also consider an alternative using the dissipation rate of the true SGS cross-helicity $H_{\text {sgs. }}$. The motivation for this will be clarified in section 2.3.2. Assuming a characteristic SGS cross-helicity time-scale $t_{H_{\mathrm{sgs}}} \sim \Delta \bar{\rho}^{1 / 4}\left|H_{\mathrm{sgs}}\right|^{-1 / 2}$ we have

$$
2 \operatorname{sgn}\left(H_{\mathrm{sgs}}\right) \nu_{\mathrm{sgs}}=\eta_{\mathrm{sgs}}=C_{1} \Delta \bar{\rho}^{-1 / 4} \operatorname{sgn}\left(H_{\mathrm{sgs}}\right) \sqrt{\left|H_{\mathrm{sgs}}\right|} .
$$

The sign is chosen for consistency with the original model.

We summarise the EV/ER models in tab. 2.2 and label them for future reference.

\section{Two Scale Direct Interaction Approximation (TSDIA) models}

An alternative to the eddy-viscosity paradigm is provided in $[110,111]$. It originates as a mean field model and has been recently applied as such to the study of magnetic reconnection $[50,108]$. Since it uses ensemble averages instead of LES filtering operators it is strictly valid only for the Reynolds part of the SGS terms (or sharp filters). The 
compressible HD work [110] suggests that the cross-term should be modelled using a scale-similarity model while the Leonard term is calculated directly from a given filter kernel. In the incompressible MHD treatment $[106,111]$ the closure of these terms is left unexplored.

The model uses the two-scale direct interaction approximation (TSDIA) [109], [106, App. A] to derive the SGS terms. The main assumption is that of isotropic and homogeneous turbulent fluctuations for the primary fields. The idea behind TSDIA [112] is to define a scale separation between slowly and quickly varying flow fields. The fast fields are expanded in spectral space and corresponding Green's functions are computed. At this point the homogeneity and isotropy of the basic fields is used and the direct interaction approximation permits the calculation of the SGS terms

$$
\begin{aligned}
\tau^{\mathrm{u} *} & =-2 \bar{\rho} \nu_{\mathrm{u}} \tilde{\mathcal{S}}^{*}, \\
\tau^{\mathrm{b} *} & =-2 \nu_{\mathrm{b}} \overline{\mathcal{M}}^{*}, \\
\mathcal{E} & =\alpha \overline{\mathbf{B}}-\beta \overline{\mathbf{J}}+\gamma \tilde{\boldsymbol{\Omega}} .
\end{aligned}
$$

We shall call $\nu_{\mathrm{b}}$ eddy-diffusivity in analogy to $\nu_{\mathrm{u}}$. For a succinct derivation of the model see [106, Appendix A]. The model predicts a relationship between the closures for the SGS stress and the EMF, namely $\beta=5 / 7 \nu_{\mathrm{u}}$ and $\gamma=5 / 7 \nu_{\mathrm{b}}$. The transport coefficients are related as follows: $\alpha$ - to the residual helicity [112]; $\beta$ - to the total SGS energy; $\gamma$ - the SGS cross-helicity. The EMF closure can be closely linked with dynamo theory, so we shall refer to the models based on eq. (2.28) as dynamo models. The $\alpha$ term is responsible for the usual $\alpha$-dynamo effect $[60,72]$ and the backscatter or reverse cascade of magnetic energy. It is present only in the case of a broken symmetry of the flow, e.g. between the velocity and magnetic fields. The $\beta$ term represents turbulent resistivity and is related to the strength of turbulence. It is the counterpart of the $\eta$-term in eq. (2.22). Finally, the $\gamma$ term is related to the cross-helicity and depends on the presence of large-scale vorticity (for more information consult [106]). The exact expressions for $\alpha, \beta$, and $\gamma$ are computationally intractable, however they suggest that the transport coefficients can be expressed via their respective quantity and some turbulence time-scale, e.g. $\beta \propto t E_{\mathrm{sgs}} / \bar{\rho}$. If the characteristic time-scale $t$ is chosen everywhere to be that of the SGS energy dissipation rate $t=E_{\mathrm{sgs}} / \epsilon \sim \Delta / \sqrt{E_{\mathrm{sgs}} / \bar{\rho}}$, where we have used the cascade approximation, we obtain the model

$$
\alpha=C_{\alpha} \Delta \frac{H_{R}}{\sqrt{E_{\mathrm{sgs}} / \bar{\rho}}}, \quad \beta=C_{\beta} \Delta \sqrt{E_{\mathrm{sgs}} / \bar{\rho}}, \quad \gamma=C_{\gamma} \Delta \frac{H_{\mathrm{sgs}}}{\sqrt{\overline{E_{\mathrm{sgs}} / \bar{\rho}}}}
$$

Note that in the context of this work we are satisfied by substituting a true expression for the residual helicity and the cross-helicity since no simple models are available for them. In practice (LES) this would correspond to solving the respective evolution equation, e.g. [105, 106, 112]. This is not really practical but falls outside the scope of this investigation.

A simpler, albeit not necessarily self-consistent, version of the model can be obtained by considering eq. (2.28). If at this point we introduce the dimensional/Prandtl's mixing 


\begin{tabular}{c|c|c|c} 
TSDIA Models & $\tau^{\mathrm{u} *}=-2 \nu_{\mathrm{u}} \overline{\mathcal{S}^{*}}$ & $\tau^{\mathrm{b} *}=-2 \nu_{\mathrm{b}} \overline{\mathcal{M}}$ & $\mathcal{E}=\alpha \overline{\mathbf{B}}-\beta \overline{\mathbf{J}}+\gamma \tilde{\boldsymbol{\Omega}}$ \\
\hline \hline Yokoi & $\nu_{\mathrm{u}}=C_{\beta} \Delta \sqrt{E_{\mathrm{sgs}} / \bar{\rho}}$ & $\nu_{\mathrm{b}}=C_{\gamma} \Delta \frac{H_{\mathrm{sgs}}}{\sqrt{E_{\mathrm{sgs}} / \bar{\rho}}}$ & $\begin{array}{c}\alpha=C_{\alpha} \Delta \frac{H_{R}}{\sqrt{E_{\mathrm{sgs}} / \bar{\rho}}} \\
\beta=5 / 7 \nu_{\mathrm{u}}, \gamma=5 / 7 \nu_{\mathrm{b}}\end{array}$ \\
\hline Constant & $\nu_{\mathrm{u}}=C_{1} \Delta^{4 / 3}$ & $\nu_{\mathrm{b}}=C_{2} \Delta^{4 / 3}$ & $\begin{array}{c}\beta=D_{1} \Delta^{4 / 3} \\
\gamma=\alpha=0\end{array}$ \\
\hline Miki & $\nu_{\mathrm{u}}=C_{2} \Delta \sqrt{E_{\mathrm{sgs}}^{\mathrm{u}} / \bar{\rho}}$ & $\nu_{\mathrm{b}}=C_{3} \Delta \sqrt{E_{\mathrm{sgs}}^{\mathrm{b}}}$ & $\begin{array}{c}\alpha=C_{1} \sqrt{E_{\mathrm{sgs}}^{\mathrm{u}} / \bar{\rho}}, \\
\beta=5 / 7 \nu_{\mathrm{u}}, \gamma=5 / 7 \nu_{\mathrm{b}}\end{array}$ \\
\hline
\end{tabular}

Table 2.3: TSDIA models.

length arguments, the Kolmogorov assumptions lead to

$$
\begin{aligned}
& \nu_{\mathrm{u}}=C_{1} \Delta^{4 / 3}, \\
& \nu_{\mathrm{b}}=C_{2} \Delta^{4 / 3} .
\end{aligned}
$$

This model is similar to eq. (2.23), but for an additional degree of freedom. Namely, the separation between $\tau^{\mathrm{u}}$ and $\tau^{\mathrm{b}}$ allows the local effective Reynolds number to vary independently based on the large scale deformations of the velocity and magnetic fields. The strength of this effect is regulated by the ratio $\left|C_{2} / C_{1}\right|$. A similar argument can be made for the EMF, but we restrict ourselves to the constant anomalous resistivity model in which $C_{\alpha}=C_{\gamma}=0$ and $\beta=D_{1} \Delta^{4 / 3}$.

If instead of constant dissipation rates we choose the kinetic and magnetic energy dissipation rates for $\nu_{\mathrm{u}}$ and $\nu_{\mathrm{b}}$ respectively, we obtain the model discussed in [59]. There the relationship between SGS stress and EMF closures is preserved, and $\alpha$ is also closed through the SGS kinetic energy. The full model takes the form

$$
\begin{array}{cc}
\alpha=C_{1} \sqrt{E_{\mathrm{sgs}}^{\mathrm{u}} / \bar{\rho}}, \quad \beta=C_{2} \Delta \sqrt{E_{\mathrm{sgs}}^{\mathrm{u}} / \bar{\rho}}, & \gamma=C_{3} \Delta \sqrt{E_{\mathrm{sgs}}^{\mathrm{b}}} \\
\nu_{\mathrm{u}}=7 / 5 \beta, & \nu_{\mathrm{b}}=7 / 5 \gamma
\end{array}
$$

As before, we summarise the TSDIA-based models in tab. 2.3 and label them for future reference.

\subsection{Sub-grid scale energy and cross-helicity models}

As we have noted, the SGS kinetic and magnetic energies enter the traces of the SGS kinetic and magnetic stress respectively and thus enter directly the momentum equation via the SGS pressure gradient $\nabla P_{\mathrm{sgs}}=\nabla\left(2 E_{\mathrm{sgs}}^{\mathrm{u}}+E_{\mathrm{sgs}}^{\mathrm{b}}\right)$. The structural models we have presented so far self-consistently prescribe the traces of the SGS stress for the whole parameter space. 
The functional models, on the other hand, historically originate from incompressible equations. Since in the incompressible regime they ignore completely the SGS energy ${ }^{6}$, alternative approaches for dealing with these terms have been devised. They take one of three forms: implicit, dynamical, geometrical. They have been carried over to the compressible case as well, since they allow for modelling of other important scalars, like the cross-helicity, which enters some of the models explicitly. We describe them in what follows and summarise the results at the end of this section in tab. 2.4.

\subsubsection{Implicit models}

The first approach to closing the SGS pressure $P_{\text {sgs }}$ is computationally and methodologically least demanding. It consists of absorbing it into the thermal pressure and treating the result within the numerical scheme, i.e. by solving for a modified pressure. This has to be consistently handled in the equation of state and (internal) energy equations.

Alternatively, the SGS pressure can be completely ignored. A reason for this comes from the resolved energy equation. The SGS pressure enters via the term $P_{\mathrm{sgs}} \nabla \cdot(\tilde{\mathbf{u}})$ (ignoring diffusion). Since this term vanishes in the incompressible regime, the SGS pressure doesn't contribute to the energy cascade and it is justified to neglect it within the EV/ER (functional) paradigm. This approach has been justified for weak/subsonic HD turbulence [30] but clearly looses ground as the SGS Mach number (the Mach number at the largest sub-grid scales) increases and the SGS pressure begins to dominate over the thermal one. In fact, in HD, [30] show that the Reynolds part of $E_{\mathrm{sgs}}^{\mathrm{u}}$, i.e half the trace of the Reynolds part of $\tau^{\mathrm{u}}$, is given by ${ }^{R} E_{\mathrm{sgs}}^{\mathrm{u}}=\frac{1}{3} \gamma M_{\mathrm{sgs}}^{2} \bar{P} \delta_{i j}$, with sub-grid Mach number $M_{\mathrm{sgs}}=\sqrt{{ }^{R} E_{\mathrm{sgs}}^{\mathrm{u}} /(\bar{\rho} \gamma R \widetilde{T})}$. So for turbulent Mach number ${ }^{7}$, (which usually is somewhat higher than the SGS one), above $\sim 0.6$ the SGS pressure is dominant and this treatment is invalid. Nevertheless, this is a widely used hypothesis in HD and has been extended to the full $E_{\mathrm{sgs}}^{\mathrm{u}}[23,73,97]$.

The cross-helicity only enters directly via the energy equation and thus it is not handled within this approach. However, most of the models above require explicit expressions for the SGS energy and cross-helicity. This leads to the next approach.

\footnotetext{
${ }^{6} \operatorname{Tr}(\overline{\mathcal{M}})=\nabla \cdot(\overline{\mathbf{B}}) \equiv 0$ and in the incompressible regime $\operatorname{Tr}(\tilde{\mathcal{S}})=\nabla \cdot(\tilde{\mathbf{u}})=0$

${ }^{7}$ This is the typical Mach number in the turbulent inertial range of scales.
} 


\subsubsection{Evolution equations and Smagorinsky approximation}

The second approach involves the evolution equations for the necessary SGS quantities. We repeat them in symbolic form:

$$
\begin{aligned}
\frac{\partial E_{\mathrm{sgs}}^{\mathrm{u}}}{\partial t} & =\mathcal{F}_{\mathrm{sgs}}^{\mathrm{u}}:-\tau \cdot \tilde{\mathcal{S}}+\epsilon_{\mathrm{sgs}}^{\mathrm{u}}, \\
\frac{\partial E_{\mathrm{sgs}}^{\mathrm{b}}}{\partial t} & =\mathcal{F}_{\mathrm{sgs}}^{\mathrm{b}}-\mathcal{E} \cdot \overline{\mathbf{J}}+\epsilon_{\mathrm{sgs}}^{\mathrm{b}}, \\
\frac{\partial H_{\mathrm{sgs}}}{\partial t} & =\mathcal{F}_{\mathrm{sgs}}^{\mathrm{ch}}-(\tau \cdot \nabla)\left(\frac{\overline{\mathbf{B}}}{\bar{\rho}}\right)-\mathcal{E} \cdot \tilde{\boldsymbol{\Omega}}+\epsilon_{\mathrm{sgs}}^{\mathrm{ch}},
\end{aligned}
$$

where $\epsilon_{\mathrm{sgs}}^{\square}$ represents the dissipation into microscopic degrees of freedom and $\mathcal{F}_{\mathrm{sgs}}^{\square}$ is a corresponding transport term. Note that the transport terms are a re-summation of the ones found in eqs. (1.32) and (1.33) in which we have included diffusion terms based on the SGS stress and EMF. This will be discussed in more detail in section 3.4. As mentioned in section 1.3 the equations require additional closures. For MHD up to four additional evolution equations have been introduced e.g. [105, 107, 109, 112]. The most accurate and computationally most challenging approach would be to close the equations and evolve them explicitly. However, the addition of the new equations can undermine the computational gains from the LES modelling, especially considering the extra computational weight of the additional closures. Nevertheless this approach has been implemented in HD e.g. [77, 79] with some success. We are not aware of MHD LES simulations where it has been fully implemented, however it has been applied for selected equations in e.g. $[50,59]$. To represent this approach in our testing we take the best-case scenario, in which the evolution equations produce accurate SGS quantities with the same error as the rest of the simulation. Thus, we substitute the true SGS terms in the deviatoric models wherever it is necessary.

Instead of solving the full evolution equations numerically, approximations can be made to try and solve them analytically. For example by reduction to algebraic equations with no unclosed terms. Most popular (e.g. [81, 106, 111]) is the Smagorinsky approximation of local balance of production and dissipation, i.e.

$$
\begin{aligned}
\epsilon_{\mathrm{sgs}}^{\mathrm{u}} & =\tau \cdot \tilde{\mathcal{S}}, \\
\epsilon_{\mathrm{sgs}}^{\mathrm{b}} & =\mathcal{E} \cdot \overline{\mathbf{J}}, \\
\epsilon_{\mathrm{sgs}}^{\mathrm{ch}} & =(\tau \cdot \nabla)\left(\frac{\overline{\mathbf{B}}}{\bar{\rho}}\right)+\mathcal{E} \cdot \tilde{\boldsymbol{\Omega}} .
\end{aligned}
$$

This amounts to assuming a statistically stationary state of homogeneous turbulence. Homogeneity here is meant in the sense that the transport terms average out to zero over the volume corresponding to a filter scale. Note that this is already required by the majority of the considered functional models so it doesn't add to the burden of approximations. 
Using the cascade approximation for the dissipation rates, $\epsilon^{f} \propto f v_{f} / \Delta$ gives an implicit algebraic equation for the SGS energies and cross-helicity in terms of the deviatoric SGS terms:

$$
\begin{aligned}
& E_{\mathrm{sgs}}^{\mathrm{u}} \propto \frac{\Delta}{v_{E_{\mathrm{sgs}}^{\mathrm{u}}}}\left(\tilde{\mathcal{S}}_{i j}^{*} \tau_{i j}^{*}+\left(P_{\mathrm{sgs}}\right) \nabla \cdot \widetilde{\mathbf{u}}\right), \\
& E_{\mathrm{sgs}}^{\mathrm{b}} \propto \frac{\Delta}{v_{E_{\mathrm{sgs}}^{\mathrm{b}}}}(\mathcal{E} \cdot \overline{\mathbf{J}}), \\
& H_{\mathrm{sgs}} \propto \frac{\Delta}{v_{H_{\mathrm{sgs}}}}\left((\tau \cdot \nabla)\left(\frac{\overline{\mathbf{B}}}{\bar{\rho}}\right)+\mathcal{E} \cdot \tilde{\Omega}\right) .
\end{aligned}
$$

To proceed we can use the eddy-viscosity approximation

$$
\begin{aligned}
E_{\mathrm{sgs}}^{\mathrm{u}} & \propto \Delta \frac{\nu_{\mathrm{sgs}}}{v_{E_{\mathrm{sgs}}^{\mathrm{u}}}} \bar{\rho}|| \tilde{\mathcal{S}} \|_{2}^{2} \\
E_{\mathrm{sgs}}^{\mathrm{b}} & \propto \Delta \frac{\eta}{v_{E_{\mathrm{sgs}}^{\mathrm{b}}}}|\overline{\mathbf{J}}|^{2} \\
\operatorname{sgn}\left(H_{\mathrm{sgs}}\right)\left|H_{\mathrm{sgs}}\right|^{3 / 2} & \propto \Delta \bar{\rho}^{1 / 4}\left(2 \bar{\rho} \nu_{\mathrm{sgs}} \tilde{\mathcal{S}}_{i j}^{*} \cdot \nabla\left(\frac{\overline{\mathbf{B}}}{\bar{\rho}}\right)+\eta \overline{\mathbf{J}} \cdot \tilde{\mathbf{\Omega}}\right),
\end{aligned}
$$

with the $L^{2}$ matrix norm given by $\|A\|_{2}=\sqrt{2 A_{i j} A_{i j}}$.

Now we can add the mixing length approximation for the viscosity/resistivity from the $\mathrm{EV} / \mathrm{ER}$ model eq. (2.24) and assume separate turbulent time-scales ${ }^{8}$ to obtain the MHD extension of the Smagorinsky model from [81, 93]

$$
E_{\mathrm{sgs}}^{\mathrm{u}} \propto \Delta^{2} \bar{\rho}|| \tilde{\mathcal{S}} \|_{2}^{2}, \quad E_{\mathrm{sgs}}^{\mathrm{b}} \propto \Delta^{2}|\overline{\mathbf{J}}|^{2}
$$

Henceforth, we shall refer to these expressions as the Smagorinsky energies. Alternatively, if we substitute $\eta_{\mathrm{sgs}}=\Delta \bar{\rho}^{1 / 4} \operatorname{sgn}\left(H_{\mathrm{sgs}}\right)\left|H_{\mathrm{sgs}}\right|^{1 / 2}=2 \operatorname{sgn}\left(H_{\mathrm{sgs}}\right) \nu_{\mathrm{sgs}}$ in the crosshelicity equation we provide the motivation for the model described in eq. (2.27), and an expression for the cross-helicity ${ }^{9}$. If instead we use the TSDIA model eq. (2.29) we need to solve a high-degree polynomial. After neglecting the $\alpha$ and $\gamma$ terms in the magnetic energy equation and the $\nu_{\mathrm{b}}$ term in the kinetic SGS energy equation, for simplicity, we obtain

$$
E_{\mathrm{sgs}} \propto C_{1} \Delta^{2} \bar{\rho}\|\tilde{\mathcal{S}}\|_{2}^{2}+C_{2} \Delta^{2}|\overline{\mathbf{J}}|^{2} .
$$

Note that this is valid only for the total SGS energy and cannot be trivially separated into magnetic and kinetic components because we have already assumed in eq. (2.29) a uniform turbulent time scale for both the kinetic and magnetic component. Furthermore, since we have neglected the $\gamma$ and $\nu_{\mathrm{b}}$ terms we cannot derive a non-trivial expression for the SGS cross-helicity consistent with eq. (2.36).

\footnotetext{
${ }^{8}$ namely, $v_{E_{\mathrm{sgs}}^{\mathrm{u}}}=\left(E_{\mathrm{sgs}}^{\mathrm{u}} / \bar{\rho}\right)^{1 / 2}, v_{E_{\mathrm{ggs}}^{\mathrm{b}}}=\left(E_{\mathrm{sgs}}^{\mathrm{b}} / \bar{\rho}\right)^{1 / 2}, v_{H_{\mathrm{sgs}}}=\left|H_{\mathrm{sgs}}\right|^{1 / 2} \bar{\rho}^{1 / 4}$

${ }^{9}$ We note in passing that eq. (2.26) gives another, rather Keplerian expression for the cross-helicity $\operatorname{sgn}\left(H_{\mathrm{sgs}}\right)\left|H_{\mathrm{sgs}}\right|^{3 / 2} \propto C_{1} \Delta^{2}\left|\tilde{\mathcal{S}}_{i j}^{*} \overline{\mathcal{M}}_{i j}\right|^{3 / 2}+C_{2} \Delta^{2}|\overline{\mathbf{J}} \cdot \tilde{\boldsymbol{\Omega}}|^{3 / 2}$.
} 


\subsubsection{Realizability condition}

Another approach for deriving the SGS energies comes from the structure of the SGS stress itself [99]. A necessary and sufficient condition that the kinetic and magnetic components of the SGS stress tensor, $\tau^{\mathrm{u}}$ and $\tau^{\mathrm{b}}$, are semi-positive definite (or realizable as coined by Vreman) is that the filtering kernel is homogeneous and non-negative in physical space (e.g. Gaussian or box, but not sharp filter). In that case the traces $E_{\mathrm{sgs}}^{\mathrm{u}}$ and $E_{\mathrm{sgs}}^{\mathrm{b}}$ are guaranteed to be non-negative in any reference frame and as such can be justifiably interpreted as energies. Additionally, this way they can be used unambiguously in all of the above models where they appear under a radical. Moreover, they can't cause artificial negative pressure through the $P_{\text {sgs }}$ term. A further property of realizable tensors is that

$$
\sum_{i \neq j}\left(f_{i i} f_{j j}-f_{i j}^{2}\right) \geq 0
$$

This can be rearranged to derive a lower bound for the trace, $\left(f_{k k}\right)^{2} \geq \frac{3}{4}\left\|f^{*}\right\|_{2}^{2}$. This lower bound can be used as a stabilisation criterion for the deviatoric model in LES (e.g. for structural models like the scale-similarity and our newly proposed one). In [97] the inequality is used instead to introduce an incompressible HD model for the SGS kinetic energy. Here we give the compressible version and apply it to the SGS magnetic energy as well

$$
E_{\mathrm{sgs}}^{\mathrm{u}} \sim \frac{\sqrt{3}}{2}\left\|\tau^{\mathrm{u} *}\right\|_{2}, \quad E_{\mathrm{sgs}}^{\mathrm{b}} \sim \frac{\sqrt{3}}{2}\left\|\tau^{\mathrm{b} *}\right\|_{2}
$$

This connects the model coefficients of the isotropic and deviatoric parts of $\tau^{\mathrm{u} *}$ and $\tau^{\mathrm{b} *}$. Since realizability only holds for $\tau^{\mathrm{u}}$ and $\tau^{\mathrm{b}}$ separately, it cannot be applied directly to obtain an expression for the total SGS energy from models for $\tau$, like the EV/ER models. Applying it to the general TSDIA model eq. (2.28) on the other hand, leads to

$$
E_{\mathrm{sgs}}^{\mathrm{u}} \sim \nu_{\mathrm{u}} \bar{\rho}\left\|\tilde{\mathcal{S}}^{*}\right\|_{2}, \quad E_{\mathrm{sgs}}^{\mathrm{b}} \sim \nu_{\mathrm{b}}\left\|\overline{\mathcal{M}}^{*}\right\|_{2} .
$$

We shall refer to these as realizable energies. Note that in order to be self-consistent we must take into account only the deviatoric part of eq. (2.28). We can now substitute these energies into eq. (2.29). If we assume that the kinetic and magnetic energy share the same coefficient of proportionality the $\beta$ term scales as

$$
\beta \propto \sqrt{\left\|\tilde{\mathcal{S}}^{*}\right\|_{2}^{2}+\frac{\left\|\overline{\mathcal{M}}^{*}\right\|_{2}^{2}}{\bar{\rho}}} .
$$

Using eq. (2.39) instead in eq. (2.31) closes that model completely.

Finally, recall that in section 2.1.4 we noted the possibility to renormalise the new model for $\tau$ with SGS energies in order to improve its stability and/or accuracy. The 


\begin{tabular}{|c|c|c|}
\hline Energy models & $E_{\mathrm{sgs}}^{\mathrm{u}}$ & $E_{\mathrm{sgs}}^{\mathrm{b}}$ \\
\hline Smagorinsky & $C_{1} \bar{\rho} \Delta^{2}|\tilde{\mathcal{S}}|^{2}$ & $C_{2} \Delta^{2}|\overline{\mathbf{J}}|^{2}$ \\
\hline Realizable & $C_{1} \bar{\rho} \Delta^{2}\left|\tilde{\mathcal{S}}^{*}\right|^{2}$ & $C_{2} \Delta^{2}|\overline{\mathcal{M}}|^{2}$ \\
\hline Scale-similarity & $C_{1} \bar{\rho}\left(\widetilde{\tilde{\mathbf{u}}^{2}}-\widetilde{\tilde{\mathbf{u}}}^{2}\right)$ & $C_{2}\left(\overline{\overline{\mathbf{B}}}^{2}-\overline{\overline{\mathbf{B}}}^{2}\right)$ \\
\hline Deconvolution & $\frac{1}{12} C_{1} \Delta^{2} \tilde{u}_{i, l} \tilde{u}_{i, l} \propto \bar{\rho}\left(|\tilde{\mathcal{S}}|^{2}+\tilde{\Omega}^{2}\right)$ & $\frac{1}{12} C_{2} \Delta^{2} \bar{B}_{i, l} \bar{B}_{i, l} \propto\left(|\overline{\mathcal{M}}|^{2}+\overline{\mathbf{J}}^{2}\right)$ \\
\hline
\end{tabular}

Table 2.4: Energy models.

realizable and Smagorinsky energies provide two additional ways to do that. Note that applying either approximation to the proposed non-linear model for $\tau^{\mathrm{u} *}$ and $\tau^{\mathrm{b} *}$ leads to energies which are of one order in $\Delta$ higher than the corresponding deviatoric model and thus should be neglected. To circumvent this, we use directly the Smagorinsky and realizable energies.

\subsubsection{Dissipation/backscatter properties of selected models}

As it was discussed, the proposed models may suffer from incorrect amount of energy dissipation. To qualify this we proceed with a brief analytical analysis of the dissipation properties of the presented models. Recall the argument behind eddy-viscosity models connecting the kinetic energy dissipation rate with the resolved rate-of-strain $\tilde{\mathcal{S}}$, namely $\epsilon_{\mathrm{sgs}}^{\mathrm{u}}=\tau \cdot \tilde{\mathcal{S}}$. Consider, a co-ordinate system which diagonalizes $\tilde{\mathcal{S}}$, say $\mathcal{R}_{\tilde{\mathcal{S}}}$. In this frame of reference only the diagonal part of $\tau$ gives a non-zero contribution to the dissipation of energy. Let the eigenvalues of $\tilde{\mathcal{S}}$ be $\lambda_{\tilde{\mathcal{S}} 1} \geq \lambda_{\tilde{\mathcal{S}} 2} \geq \lambda_{\tilde{\mathcal{S}} 3}$. In general, we can decompose the SGS stress tensor into a term which shares an eigenbasis with $\tilde{\mathcal{S}}$ and a residual. In $\mathcal{R}_{\tilde{\mathcal{S}}}$ these are simply the diagonal and off-diagonal components $\tau=\tau^{\tilde{\mathcal{S}} \text { eigen }}+\tau^{\tilde{\mathcal{S}}_{\text {perp }}}$. Clearly then $\tau_{i j}^{\tilde{\mathcal{S}} \text { perp }} \tilde{\mathcal{S}}_{i j} \equiv 0$, while

$$
\tau_{i j} \tilde{\mathcal{S}}_{i j}=\tau^{\tilde{\mathcal{S}} \text { eigen }} \tilde{\mathcal{S}}_{i j}=\lambda_{\tilde{\mathcal{S}} 1} \tau_{11}+\lambda_{\tilde{\mathcal{S}} 2} \tau_{22}+\lambda_{\tilde{\mathcal{S}} 3} \tau_{33}
$$

For the general eddy-viscosity model $\tau^{\mathrm{u}}=-\nu_{\text {turb }} \tilde{\mathcal{S}}$ eq. (2.41) becomes $\epsilon_{\mathrm{EV}}^{\mathrm{u}}=\nu_{\text {turb }}\left(\lambda_{\tilde{\mathcal{S}} 1}^{2}+\lambda_{\tilde{\mathcal{S}} 2}^{2}+\lambda_{\tilde{\mathcal{S}} 3}^{2}\right)$. This once more clearly demonstrates the dissipative nature of the eddy-viscosity model, since the bracket is always non-negative, this model is independent of the local anisotropy of the flow and can only dissipate energy from all types of SGS flows. 
In contrast our model has the shape $\tau^{\mathrm{u}}=f(\tilde{\mathcal{S}}+\tilde{\Omega})(\tilde{\mathcal{S}}+\tilde{\Omega})$ (with a non-negative scalar modulator field $f$ ). Performing the same computation for the $\tilde{\mathcal{S}} \tilde{\mathcal{S}}$ term we obtain $\epsilon_{\tilde{\mathcal{S}} \tilde{\mathcal{S}}}=-f\left(\lambda_{\tilde{\mathcal{S}} 1}^{3}+\lambda_{\tilde{\mathcal{S}} 2}^{3}+\lambda_{\tilde{\mathcal{S}} 3}^{3}\right)$. This time the strength is enhanced (cubic versus square powers), however the signature is not definite. For instance, a negative eigenvalue leads to a backscatter effect. This identifies one of the sources of backscatter in the model, net stretching along an eigenvector of $\tilde{\mathcal{S}}$. The vorticity $\tilde{\Omega} \tilde{\Omega}$ term has a similar behaviour, but with the opposite sign

$$
\epsilon_{\tilde{\Omega} \tilde{\Omega}}=f\left(\lambda_{\tilde{\mathcal{S}} 1}\left(\mu_{\tilde{\Omega} 2}^{2}+\mu_{\tilde{\Omega} 3}^{2}\right)+\lambda_{\tilde{\mathcal{S}} 2}\left(\mu_{\tilde{\Omega} 1}^{2}+\mu_{\tilde{\Omega} 3}^{2}\right)+\lambda_{\tilde{\mathcal{S}} 3}\left(\mu_{\tilde{\Omega} 1}^{2}+\mu_{\tilde{\Omega} 2}^{2}\right)\right)
$$

where $\left(\mu_{\tilde{\Omega} 1}, \mu_{\tilde{\Omega} 2}, \mu_{\tilde{\Omega} 3}\right)$ are the components of the resolved vorticity in $\mathcal{R}_{\tilde{\mathcal{S}}}$. Here, the main conclusion is that strain along the vorticity axes doesn't influence the energy flux either way. Additionally, compression in the plane of the vortex results in backscatter, while stretching in the same plane enhances the dissipation. Putting both together

$$
\epsilon_{\mathrm{nl}}^{\mathrm{u}}=-f \sum_{i=1,2,3} \lambda_{\tilde{\mathcal{S}} i}\left(\lambda_{\tilde{\mathcal{S}} i}^{2}+\mu_{\tilde{\Omega} i}^{2}-\widetilde{\omega}^{2}\right)
$$

where $\widetilde{\omega}=\sqrt{\widetilde{\Omega}^{2}}$ is the resolved enstrophy. So, our model with $f \geq 0$ predicts dissipation $\left(\epsilon_{\mathrm{nl}}^{\mathrm{u}}>0\right)$ from compressive directions (without loss of generality $\lambda_{\tilde{\mathcal{S}} 1}<0$ ) when $\lambda_{\tilde{\mathcal{S}} 1}^{2}>$ $\mu_{\tilde{\Omega} 2}^{2}+\mu_{\tilde{\Omega} 3}^{2}$, i.e. when the compression in that direction is stronger than the vorticity in the orthogonal plane and conversely from stretching directions $\left(\lambda_{\tilde{\mathcal{S}} 1}>0\right)$ when the orthogonal vorticity is stronger. From the point of view of a vortex, a strong compression or weak stretching in the plane of the vortex enhances the dissipation and conversely a weak compression or strong stretching leads to a backscatter. Putting this into an alternative form

$$
\epsilon_{\mathrm{nl}}^{\mathrm{u}}=-f \sum_{i=1,2,3} \lambda_{\tilde{\mathcal{S}} i}^{3}+\mu_{\tilde{\Omega} i}^{2}\left(\lambda_{\tilde{\mathcal{S}} i}-\operatorname{Tr}(\tilde{\mathcal{S}})\right)
$$

reveals a different view point. Compression always leads to dissipation, stretching a vortex in its plane leads to backscatter, perpendicular to it has no effect.

The same analysis can be repeated for $\tau^{\mathrm{b}}=f(\overline{\mathcal{M}}+\bar{J})(\overline{\mathcal{M}}+\bar{J})+\frac{2}{3} E_{\mathrm{sgs}}^{\mathrm{b}} \mathrm{I}$. This leads to

$$
\epsilon_{\mathrm{nl}}^{\mathrm{b}}=-f \sum_{i=1,2,3} \lambda_{\tilde{\mathcal{S}} i}\left(\mu_{\bar{J} i}^{2}-\bar{J}^{2}\right)+\lambda_{\tilde{\mathcal{S}} i}\left(\overline{\mathcal{M}}_{i}^{2}+\frac{2}{3} E_{\mathrm{sgs}}^{\mathrm{b}}\right)
$$

where $\overline{\mathcal{M}}_{i}^{2}=\sum_{j} \overline{\mathcal{M}}_{i j}^{2}$. The current term $\bar{J} \bar{J}$ has the same shape as the vorticity term above, while the magnetic shear along with the magnetic energy give always nonnegative contributions. So, magnetic shear and SGS magnetic energy always enhance the flow action (dissipation form compression, backscatter from stretching) because the last bracket is always non-negative. On the other hand, large-scale currents lead to backscatter when flowing perpendicular to compression regions and dissipation when 
perpendicular to stretching/dilatation regions. Again, currents flowing along the compression/dissipation direction have no effect on the SGS energy.

Finally, looking at the magnetic energy dissipation $\epsilon^{\mathcal{E}}=-\mathcal{E} \cdot \overline{\mathbf{J}}$, the topology is simpler, due to the anti-symmetry of the current tensor $\bar{J}$. Clearly, there is backscatter when the electromotive force is aligned with the large-scale currents and dissipation happens in cases of anti-alignment. For the classical anomalous resistivity closure, $\mathcal{E}=\beta \bar{J}$, the switch between turbulent dynamo and anomalous resistivity regimes happens via the sign of $\beta$. In the case of the 'incompressible' term of the proposed model $\mathcal{E}=f \epsilon_{i j k} \tilde{u}_{j, l} \bar{B}_{k, l}$, we get somewhat more complicated behaviour, based on the relative alignment of the eigenvectors of resolved deformation tensors

$$
\epsilon_{\mathrm{nl}}^{\mathcal{E}} \propto \epsilon_{i j k} \tilde{\mathcal{S}}_{j l} \overline{\mathcal{M}}_{k l} \bar{J}_{i}+\overline{\mathbf{J}}^{\mathrm{T}} \cdot \tilde{\mathcal{S}} \cdot \overline{\mathbf{J}}-\tilde{\Omega}^{\mathrm{T}} \cdot \overline{\mathcal{M}} \cdot \overline{\mathbf{J}}-\overline{\mathbf{J}}^{2} \operatorname{Tr}(\tilde{\mathcal{S}}) .
$$

Each of the terms can contribute to either regime, depending on the local alignment of eigenvectors, as for the SGS stress. The last term is reminiscent of the anomalous resistivity closures above, however it is modulated here by the level of compression of the resolved flow, as opposed to energetic effects. In fact, based on this decomposition we remark on a link between the proposed model and the TSDIA family - decomposing the resolved velocity and magnetic field Jacobians into symmetric and anti-symmetric components and substituting it into the proposed model for $\mathcal{E}$ produces a four-term expression with similar structure to the TSDIA version. There is a key difference however: the proposed model has tensor-valued modulators instead of the scalar-based $\alpha, \beta, \gamma$.

What is left is to compare the models with the behaviour of SGS turbulence directly is this correlation between enhanced/suppressed SGS energy dissipation and the SGS tensor on the one hand, and the large scale Jacobians on the other hand, observed or not?

\subsection{Final remarks}

Before we conclude this discussion we need to clarify a few points.

Firstly, as has been shown, most closures come with a set of coefficients which need to be determined externally. They indicate the strength of the effect that the closure is supposed to model. These coefficients can be determined in several ways.

In LES simulations often a dynamic procedure is used based on Germano's identity [44]. This involves a scale-similarity hypothesis according to which the coefficients should be independent of the filter scale. A second, test filter is introduced. Computing the SGS terms on the test filter scale and using the scale-similarity hypothesis one can obtain closed form expressions for the coefficients only in terms of quantities of the filter (resolved) scale. This has the added flexibility to be computable locally or globally, so spatial variations in the coefficients can be included, if desired. It should be noted that this procedure is quite often very computationally expensive, especially for non-spectral codes with large test filter scale.

For the purposes of this work the particular values of the coefficients are not critical, rather the assumption that they are constant is. In other words, the closures ought to 
include all the required functional dependence explicitly and the influence of the filter scale should already be incorporated. Additionally, we shall investigate how the coefficients change with time and plasma parameters. So we compute them by maximising the correlation between closure and real term. This has the added benefit that if the coefficients turn out to be independent of plasma parameters and temporal evolution they can directly inserted into LES simulations, greatly reducing the computational cost.

Secondly, we discuss the possibility to combine models in order to benefit from their different strong points. It has been found $[1,18]$ that the functional type of models lead to too much dissipation (even if backscatter is allowed as in eq. (2.25)) and tend to have poor local correlations with the SGS terms. On the other hand, structural models like the scale-similarity and deconvolution ones maintain good local correlations but can become unstable in regions of strong gradients and in general under-predict the energy dissipation rate. Thus it has been suggested e.g. [9, 14, 43, 77, 83] to combine the two types of models to hopefully benefit from both good points and diminish the bad ones. This has been applied with remarkable success $[51,77,79]$ to compressible HD in the context of astrophysical turbulence. Since the energy dissipation rate cannot be investigated in too much detail with a priori testing, we restrict ourselves to only a few sample mixed models, which hopefully will illustrate the general trend. Namely we additionally will test two linear combinations between the proposed non-linear model and the model described by eq. (2.31). The first combination uses the true SGS energies, the second - the realizable energies, i.e. eq. (2.39). We use the renormalized version of the proposed model to gauge whether the renormalization itself provides sufficient stabilisation.

Note that in hydrodynamics combining the renormalized form of the non-linear model with and the EV one is necessary in order to initialize the turbulence cascade. In MHD turbulence this can happen spontaneously via the magnetic channel as well, so the combination is not strictly required from an a priori point of view.

Lastly, a comment on smoothness. It has been pointed out that shocks are a strong source of turbulence. However since shocks are inherently a particle and not a fluid phenomenon, SGS fluid models usually deal with them poorly. Almost all models we have described deal with derivatives of the resolved fields. Clearly, they become inapplicable in regions where these derivatives cease to exist. One trivial solution we see is to deal with shocks in analogous way to DNS. Namely, apply the filtering operation to the derivation of the Rankine-Hugenoit conditions (or their compressible MHD extension) rather than the fluid equations themselves. This should also be applicable to reconnection regions, where the magnetic field can formally experience a discontinuity. In practice, due to the discretization of the differential operator a discontinuity-capturing scheme must be employed which identifies the non-smooth regions. The usual ones employed in DNS will probably need to be modified due to the effects induced by the SGS model, but we leave this as future a posteriori work.

To summarise, we presented a new structural model for compressible MHD based on series expansion of the filter transfer function. This was followed by an overview of the main types of alternative closures. We followed their derivations and identified the main assumptions and approximations made. We finished with a discussion of 
SGS energy and cross-helicity closures. In the next chapter we perform a model-free analysis of the data presented in section 1.5 in regard to the model assumptions and in anticipation of the direct model validation in chapter 4 . 



\section{Analysis of the compressible MHD LES equations and hypothesis testing}

\subsection{Analysis of the primary equations}

Here we shall discuss some properties of the MHD LES equations relevant to SGS modelling. We begin with an analysis of the effect of the SGS terms in the primary LES equations. This will give insight into the validity of the various hypotheses on which the models from the previous sections are based, e.g. homogeneity and isotropy, local balance, scale-similarity, et cetera. We continue with a topological analysis of the SGS stress. This provides more detailed information about the topological properties that an SGS closure better satisfy. Finally we discuss the role of the SGS terms in the resolved and SGS energy and cross-helicity equations, specifically with respect to the local equilibrium hypothesis and the cascade paradigm.

The SGS terms are obtained by filtering the raw data at filter scale $k=16, \Delta=$ 16 , which is well within the inertial range as discussed in section 1.5.2. This should minimize the contamination from forcing and numerical errors from the the integral and dissipation ranges respectively, leaving the turbulent dynamics to dominate.

For ease of reading we give a short explanation of the types of plots which will be encountered in this chapter. For each considered term, say $\tau$, we can compute the distribution of some quantity $f(\tau)$ over the simulation domain at each given time. Because we are working with discrete data, we compute a histogram of the relative number of cells (or data points). This is illustrated in fig. 3.1a. To summarise the dependence of $f$ on time and plasma parameters with minimum loss of information we proceed in one of two directions depending of whether $f$ is associated with a norm (i.e. some sort of magnitude) or an angle.

In the former case we note the position of the median and the characteristic width of the distribution as measured by the interquartile, i.e. the region between the $25 \%$ and $75 \%$ percentiles. We then plot those as a function of time resulting in fig. $3.1 \mathrm{~b}$. For bimodal distributions we perform this for each branch of the distributions separately. For the quantities we consider the two branches are clearly separated as a positive and a negative one. To gauge the relationship to plasma parameters we compress the time evolution of each simulation into a thin bar. The darker regions in the bar correspond to the extent of time variation of the median and the edges of the interquartile. These constructs are ordered for each simulation by a relevant parameter, e.g. the RMS sonic Mach number. The final result is shown in fig. 3.1c. 
Note that we try to order the simulations by the parameter which appears to demonstrate the most consistent trend, whenever such a trend is evident. While we attempt to draw physical inferences from the ordering parameter we make no claims to causation, only relation. The number of simulations compared to the number of degrees of freedom in parameter space is insufficient for more definitive conclusions. We note trends related to $\beta_{\mathrm{p}, 0},\left\langle\mathrm{M}_{\mathrm{s}}{ }^{\mathrm{rms}}\right\rangle,\left\langle\left(\mathrm{M}_{\mathrm{a}}\right)_{\mathrm{m}}\right\rangle,\left\langle\beta_{\mathrm{p}}\right\rangle=\left\langle 2\left(\mathrm{M}_{\mathrm{a}}{ }^{\mathrm{rms}}\right)^{2} /\left(\mathrm{M}_{\mathrm{s}}{ }^{\mathrm{rms}}\right)^{2}\right\rangle,\left\langle\left(\mathrm{M}_{\mathrm{s}}\right)_{\mathrm{m}}+\left(\mathrm{M}_{\mathrm{a}}\right)_{\mathrm{m}}\right\rangle$ and $\left\langle\sqrt{\left(\mathrm{M}_{\mathrm{s}}^{\mathrm{rms}}\right)^{2}+\left(\mathrm{M}_{\mathrm{a}}^{\mathrm{rms}}\right)^{2}}\right\rangle$. In this a time average over the statistically stationary period is denoted with angular brackets \langle\rangle , while the spatial mean and root mean square (RMS) over the simulation domain are given by ()$_{\mathrm{m}}$ and ()$^{\mathrm{rms}}$ respectively. Note that we don't use the local plasma beta parameter, but an estimate based on $\left\langle\mathrm{M}_{\mathrm{a}}{ }^{\mathrm{rms}}\right\rangle$ and $\left\langle\mathrm{M}_{\mathrm{s}}^{\mathrm{rms}}\right\rangle$.

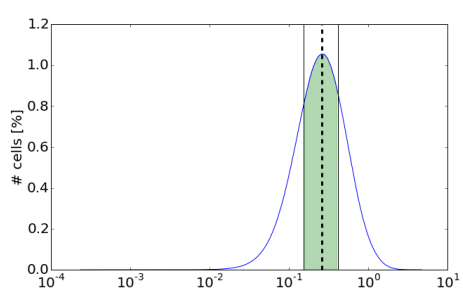

(a)

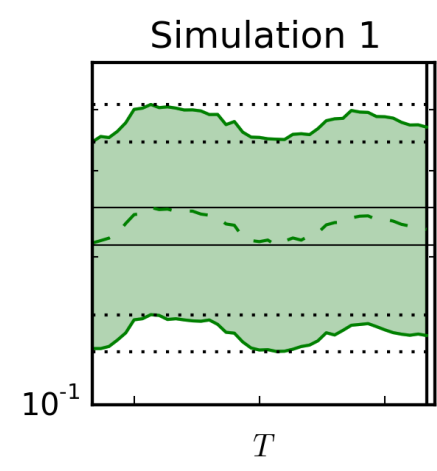

(b)

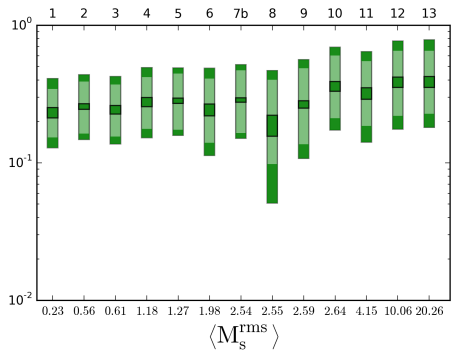

(c)

Figure 3.1: Illustration of combining single-time magnitude histograms. a) Histogram of a quantity $f$ over the simulation domain at one time snapshot, with median and interquartile; b) Evolution of the distribution of $f$ with time (in units of eddy turnover time). The median is depicted by dashed line, the shaded region is the variation of the interquartile, the solid and dotted horizontal lines show the extent of variation of the median and the interquartile respectively. c) Summary of the statistics of $f$ for different plasma parameters. Each vertical bar represents one simulation. The darker boxes depict the time variation of the median (central box) and the interquartile (outer boxes). The simulations are ordered by a plasma parameter, here $\left\langle\mathrm{M}_{\mathrm{s}}{ }^{\mathrm{rms}}\right\rangle$ (shown on the lower axis). The top axis shows the simulation number for reference.

For angular diagnostics we begin again with the distribution of a quantity over the simulation domain for one particular snapshot. We choose the quantity to be the cosine of the angle between two vectors. The treatment here is simpler since the range of the distribution is fixed to $[-1,1]$. This means that we can use fixed and regularly spaced bins (with bin width of $\sim 1 / 180$ ). We can also easily convert the histogram to a probability density function, which is in principle independent of the binning. An example is given in fig. 3.2a. In contrast to the magnitude plots, there is little variation of the angular distributions throughout the statistically stationary period and also across simulations. In fig. $3.2 \mathrm{~b}$ we show one such angular distributions for all available data. To gain a measure of the characteristic distribution we highlight the 
median value within each bin for all data. The final result is presented in fig. 3.2c. This shows that the relative orientation of SGS and resolved vectors can be a robust criterion for the goodness of fit of a model, specifically for the turbulence anisotropy. Since the analysed data represents homogeneous and isotropic turbulence, no strong preferential alignment is expected for the SGS terms. Thus there is little geometrical structure to be matched and purely isotropic models might do just as well as ones with the correct anisotropy.

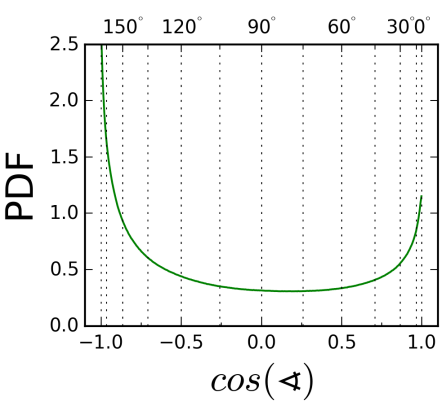

(a)

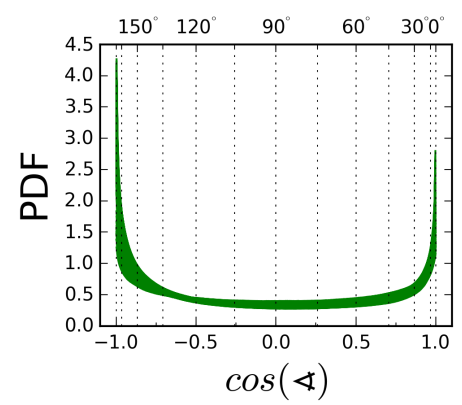

(b)

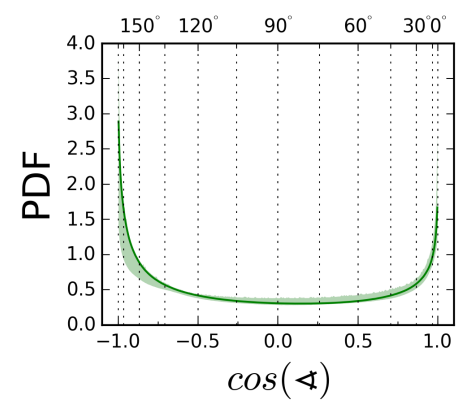

(c)

Figure 3.2: Illustration of combining single-time angular histograms into summary plots. a) Distribution of the cosine of the angle between two vectors $\mathbf{x}$ and $\mathbf{y}$ over the simulation domain at a fixed time. b) The distribution from the previous panel plotted for all simulations and at all times within the statistically stationary period. c) The distribution from the previous panel with the median in each bin shown in the solid line and the entire range of variation shown by the shaded region.

In a sense the angular and magnitude plots provide mutually orthogonal views on the data. That is to say that the shaded region in a magnitude plot represents variation within the simulation domain and the time and simulations are represented along the abscissa while in an angular plot these are reversed: the shaded region represents variation with respect to time and simulation, while the abscissa gives the variation with the simulation domain. With this we are ready to proceed to the analysis of the SGS terms.

\subsection{Induction equation and the EMF}

For simplicity, we begin the analysis with the induction equation since it contains only two vectorial terms $-\nabla \times \mathcal{E}$ and $\nabla \times(\tilde{\mathbf{u}} \times \overline{\mathbf{B}})$. In fig. 3.3 we compare the relative sizes of the EMF $|\mathcal{E}|$ and its resolved counterpart $|\tilde{\mathbf{u}} \times \overline{\mathbf{B}}|$ as well as their contributions to the induction equation, i.e. $|\nabla \times \mathcal{E}|$ and $|\nabla \times(\tilde{\mathbf{u}} \times \overline{\mathbf{B}})|$. Locally the EMF is about $5 \%-20 \%$ of its resolved counterpart with a median around $15 \%$, while its effect in the induction equations is somewhat stronger $-\sim 10 \%-80 \%$ with a median around $40 \%$. In other words, the SGS term is sub-dominant but far from negligible to the induction dynamics. We note that the statistics of $|\mathcal{E}| /|\tilde{\mathbf{u}} \times \overline{\mathbf{B}}|$ separates the data into two groups with respect to $\left\langle\mathrm{M}_{\mathrm{a}}\right\rangle$ which are differentiated both in the median value 
and the overall distribution (the whole bar is shifted to higher values at higher $\left\langle\mathrm{M}_{\mathrm{a}}\right\rangle$ ). The differentiated counterpart $|\nabla \times \mathcal{E}| /|\nabla \times(\tilde{\mathbf{u}} \times \overline{\mathbf{B}})|$ instead decreases with the timeaveraged $\left\langle\beta_{\mathrm{p}}\right\rangle$. The time variation is relatively small, as expected for a statistically stationary period.

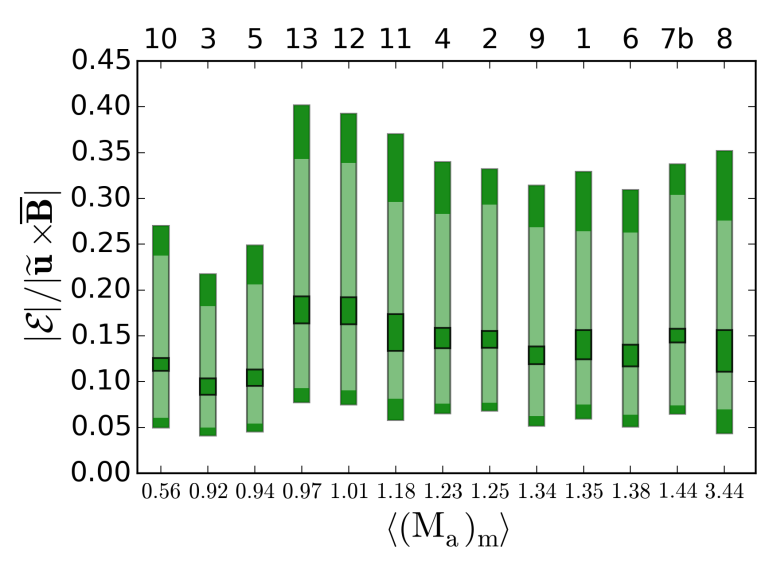

(a)

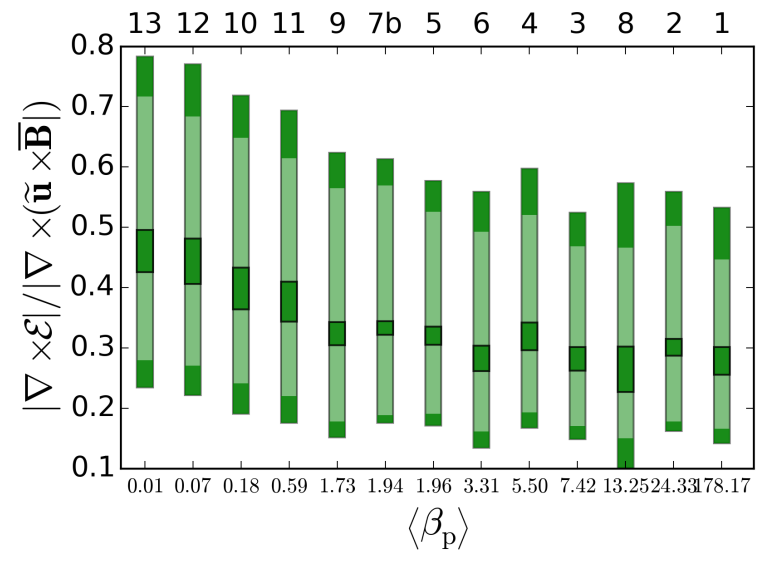

(b)

Figure 3.3: Comparison of the relative size of a) the EMF to its resolved counterpart $|\tilde{\mathbf{u}} \times \overline{\mathbf{B}}|$, b) their contributions to the induction equation: $|\nabla \times \mathcal{E}|$ and $|\nabla \times(\tilde{\mathbf{u}} \times \overline{\mathbf{B}})|$.

Next we consider the geometrical distribution of the two pairs of terms. Figure 3.4a shows that in the developed turbulence regime the distribution of the angles is almost uniform. There is a slight preference for collinearity for the EMF. In the induction equation however, this is reduced and the terms act preferentially in opposite directions. Figure $3.4 \mathrm{~b}$ shows the same terms in the initial stages of the simulations where the turbulent magnetic field is increasing to saturation level (presumably by some sort of turbulent dynamo mechanism). While we cannot make conclusive statements due to the influence of initial conditions we note that qualitatively the medians follow the same trends. The variations are of course much greater. Furthermore, there are times/snapshots with significant tendency towards alignment between $\nabla \times \mathcal{E}$ and $\nabla \times$ $(\tilde{\mathbf{u}} \times \overline{\mathbf{B}})$.

Les us expand this examination to other resolved vector fields in addition to $\tilde{\mathbf{u}} \times \overline{\mathbf{B}}$. As we saw in the previous chapter the EMF is usually modelled as a combination of resolved vector fields, each modulated by a scalar function. To begin with there are the vector fields on which the ER and TSDIA models eq. (2.28) are built, namely $\{\overline{\mathbf{B}}, \overline{\mathbf{J}}, \tilde{\boldsymbol{\Omega}}\}$. If $|\mathcal{E}|$ is the key parameter in LES we expect that the EMF closure matches to some extent its statistical distribution. Since this is sensitive to the statistics of the scalar pre-factors and requires the examination of specific model, it is deferred to the next chapter. Here instead, we prefer to take a more general approach. We investigate which resolved vectors are preferentially aligned with $\mathcal{E}$ (respectively $\nabla \times \mathcal{E}$ ). We expand the TSDIA set to include the simplest resolved vector fields accessible in an MHD simulation ${ }^{1}$ :

\footnotetext{
${ }^{1}$ At this stage we ignore the fact that the $\mathcal{E}$ is a pseudo-vector - this symmetry requirement can be accommodated by a suitable choice of scalar or pseudo-scalar modulating field.
} 


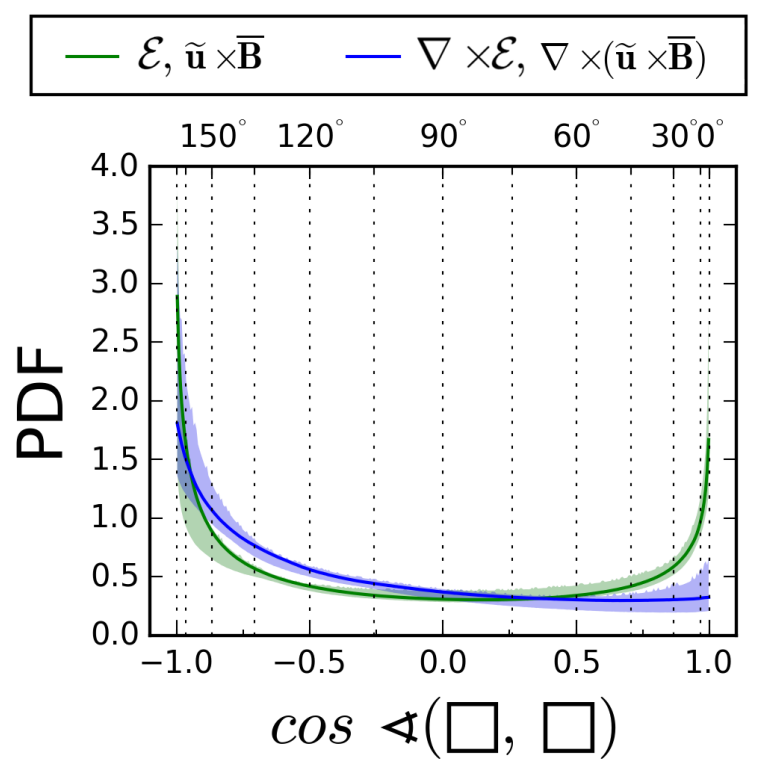

(a) $t \in[2,5] T$

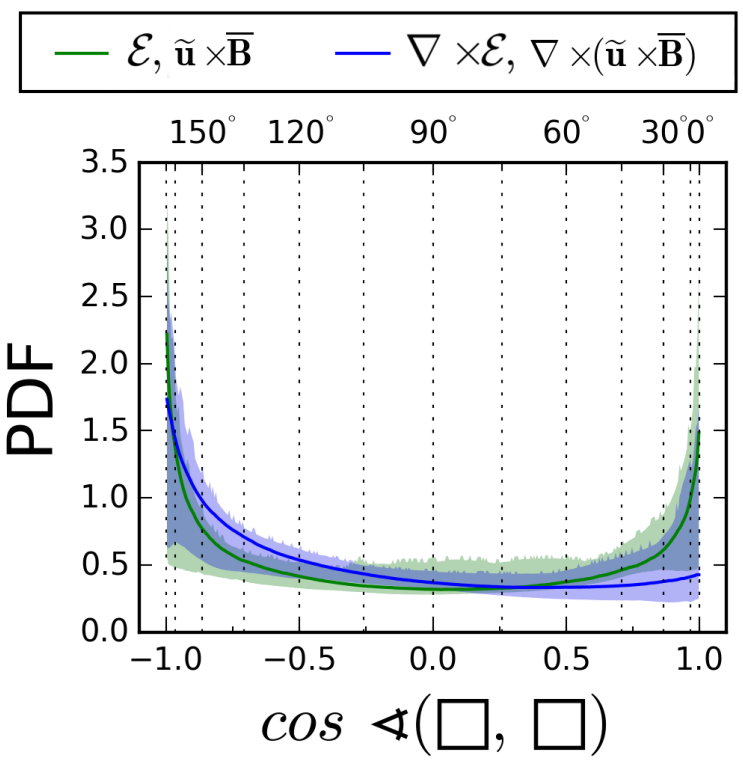

(b) $t \in[0,2] T$

Figure 3.4: Comparison of the geometry of the EMF and its resolved counterpart $\tilde{\mathbf{u}} \times \overline{\mathbf{B}}$ and their contributions to the induction equation for a) the developed turbulence regime $t \in[2,5] T, \mathrm{~b})$ the initial stages of growth of the turbulent magnetic field $t \in[0,2] T$.

$\{\tilde{\mathbf{u}}, \overline{\mathbf{B}}, \overline{\mathbf{J}}, \tilde{\boldsymbol{\Omega}}, \tilde{\mathbf{u}} \times \overline{\mathbf{B}}, \overline{\mathbf{J}} \times \tilde{\boldsymbol{\Omega}}, \tilde{\mathbf{u}} \times \overline{\mathbf{J}}, \tilde{\mathbf{u}} \times \tilde{\boldsymbol{\Omega}}, \overline{\mathbf{B}} \times \overline{\mathbf{J}}, \overline{\mathbf{B}} \times \tilde{\Omega}, \nabla \times(\tilde{\mathbf{u}} \times \overline{\mathbf{B}})\} . \quad$ The TSDIA fields along with the ones which exhibit the strongest sensitivity to the directionality of $\mathcal{E}$ are plotted in fig. 3.5a. From the shallowness of the distributions (a randomly aligned field would exhibit a constant distribution at 0.5 ) it is clear that no simple resolved vector field has the appropriate geometrical configuration. This implies that none of the one-term eddy-resistivity closures based on eq. (2.22) can capture the orientation of the EMF. This is not too surprising since they were never designed to achieve this. However, it is a first indication that they may suffer in circumstances where the EMF orientation and hence anisotropy plays a significant role. This conclusion can be extended to any one-term model aligned with any of the resolved vectors listed above.

The TSDIA models provide the next logical step by introducing a linear combination of vector fields. Whether this solves the alignment problem again depends also on the details of the scalar modulators. Interestingly though, the resolved magnetic field $\overline{\mathbf{B}}$ has a weak preference to orthogonality with the EMF. Therefore, it can serve primarily as an error-cancelling contribution of the orthogonal components of the other vector fields with little intrinsic effect to the EMF itself. Furthermore, we note that the resolved current and vorticity are largely insensitive to the orientation of the EMF. In fact much better alignment is exhibited by the quadratic terms $\overline{\mathbf{B}} \times \overline{\mathbf{J}}, \overline{\mathbf{B}} \times \tilde{\boldsymbol{\Omega}}$ and $\overline{\mathbf{J}} \times \tilde{\boldsymbol{\Omega}}$. The latter even doesn't have the strong \pm symmetry shown by the rest. This would place it as a structurally preferred candidate in comparison with the resolved current, for instance. As we are considering functional models however, it is more pertinent to examine the alignment of the vectors with $\nabla \times \mathcal{E}$. The ones exhibiting the highest sensitivity to the orientation of $\nabla \times \mathcal{E}$ are shown in fig. 3.5b. Note that only the resolved 


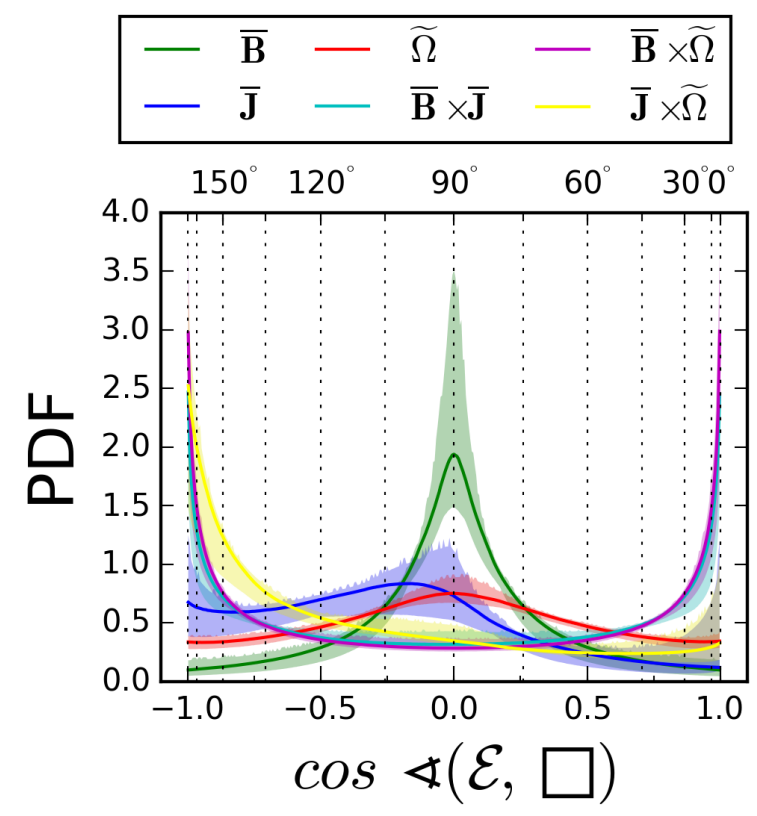

(a)

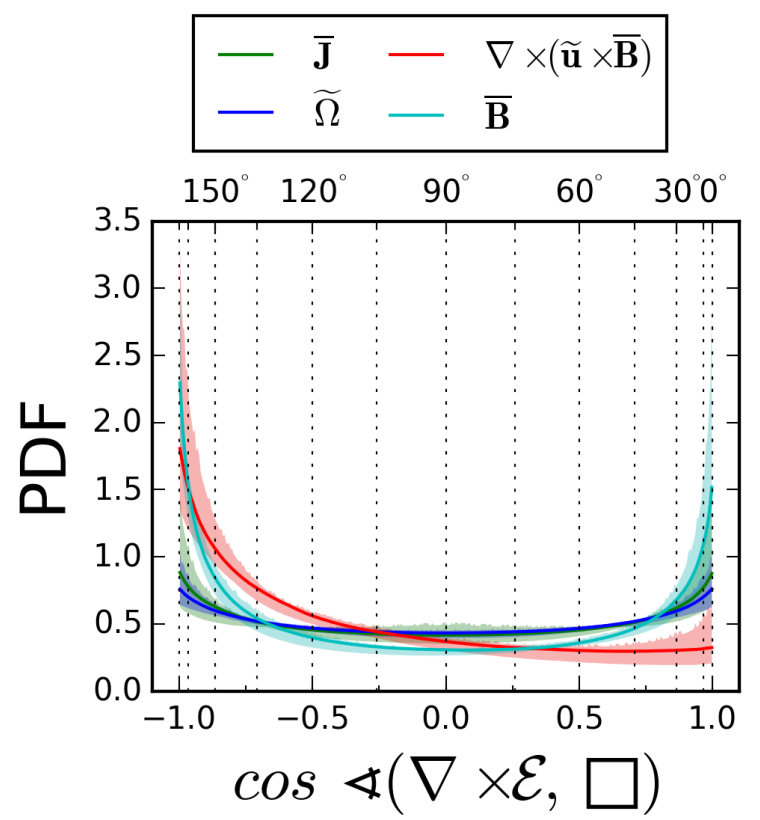

(b)

Figure 3.5: Geometrical alignment with closure-candidate vector fields for a) $\mathcal{E}$ and b) $\nabla \times \mathcal{E}$

counterpart of the EMF appears in both panels of fig. 3.5 and no other quadratic vector does. In general, we note that alignment is not preserved through differentiation. As an example the resolved current $\overline{\mathbf{J}}=\nabla \times \overline{\mathbf{B}}$ is now insensitive to $\nabla \times \mathcal{E}$. This implies that the amount of information about the SGS scales contained in the resolved vectors is purely local and does not extend to the range of our differentiation stencil (5-point per dimension). The curls of the TSDIA vectors are not included in this investigation, apart from $\overline{\mathbf{J}}(\leftarrow \overline{\mathbf{B}})$. The reason is that the higher order derivative terms would expand the possible set of vector combinations to an unreasonable number. So it is possible that the alignment issue is resolved in the induction equation by the contributions of $\nabla \times \overline{\mathbf{J}}$ and $\nabla \times \tilde{\Omega}$. We shall see that this will not help much with the contribution to the energy and cross-helicity cascade, on which the TSDIA models are founded. But first we take a look at the SGS stress and the momentum equation.

\subsection{Momentum equation and the SGS stress}

For brevity let us denote the resolved counterpart of the SGS stress with $\theta$, thus for instance the resolved kinetic stress is $\theta_{i j}^{\mathrm{u}}=\bar{\rho} \tilde{u}_{i} \tilde{u}_{j}$. Then the total momentum flux, or total force, acting at a point can be written as $\mathcal{F}^{\text {mom }}=\nabla \cdot(\theta+\tau)+\nabla \bar{P}$ and the LES momentum equation takes the form

$$
\frac{\partial \bar{\rho} \widetilde{\mathbf{u}}}{\partial t}+\mathcal{F}^{\text {mom }}=0
$$


Now consider the relevance of the SGS force with respect to $\mathcal{F}^{\text {mom }}$. Figure 3.6a shows the resolved $(\nabla \cdot \theta)$, sub-grid scale $(\nabla \cdot \tau)$ and thermal $(\nabla \bar{P})$ forces normalised by $\left|\mathcal{F}^{\text {mom }}\right|_{\text {mean }}$, i.e. the total momentum flux averaged spatially over the simulation domain. In the plot a term's absolute variation throughout the simulation domain is represented by the length of the coloured bar. Thus we see that the variation of all the terms increases with increasing $\left\langle\mathrm{M}_{\mathrm{S}}{ }^{\mathrm{rms}}\right\rangle$ - a manifestation of the more varied dynamics which makes modelling the supersonic regime so much more difficult ${ }^{2}$. At the same time, the characteristic value of the SGS and thermal forces, as determined by their median, decreases with the sonic Mach number. As expected the thermal pressure is negligible for highly supersonic simulations. The SGS forces however remain relevant throughout the whole parameter range. Figure 3.6b shows the same forces normalised by the local value of $\left|\mathcal{F}^{\mathrm{mom}}\right|$. We see that locally the resolved forces are the dominant contribution for $\left\langle\mathrm{M}_{\mathrm{s}}{ }^{\mathrm{rms}}\right\rangle \gtrsim 2$ with the SGS and thermal forces providing the necessary counterbalance. For smaller sonic Mach numbers $\left(\left\langle\mathrm{M}_{\mathrm{s}}^{\mathrm{rms}}\right\rangle \sim 1\right)$ the total force is produced primarily by the cancellation between $\nabla \cdot \theta$ and $\nabla \bar{P}$ (since the medians of both of these terms exceed unity while the $\nabla \cdot \tau$ distribution remains below 1$)$. Importantly, the relative SGS contribution increases with decreasing sonic Mach and in the subsonic regime can locally exceed the total force. This implies that in the subsonic regime the net force is the result of the combination of three comparable terms. We confirm this by examining the alignment of the SGS forces with the resolved and thermal ones in fig. 3.7. While the distributions are relatively flat, as expected for homogeneous and isotropic turbulence, the resolved forces are preferentially anti-aligned with the SGS ones. The thermal force exhibits an interesting change of behaviour with the RMS sonic Mach number. In the subsonic regime the SGS force is insensitive to the direction of the thermal pressure force. However, in the trans- and super sonic regime, when the thermal force becomes less important there is a tendency of alignment.

Next let us examine the constituents of the resolved and SGS forces. Figure 3.8a shows that the isotropic parts dominate over the deviatoric ones rather robustly. Recall that simulations 8-10 have a predominantly compressive forcing while in the rest of the simulations the compressive and solenoidal modes have equal strength. This distinction does not seem to affect the SGS scales, i.e. there has been sufficient mode mixing by the time the energy has cascaded to the filter scale. This demonstrates in a new way that the details of the forcing do not influence the SGS results and reinforces the choice of filter scale. We also note that the deviatoric components have much more compact distributions than the isotropic ones independent of the compressibility of the flow. Figure 3.8b shows that the orientation of the SGS force however is much better approximated by the deviatoric components $\nabla \cdot \tau^{*}$. The isotropic component is quite insensitive to the direction of the deviatoric and total SGS force. This combination of size-versus-angle features supports the energy-based re-normalisation approach to the proposed non-linear closures from section 2.1.4. Since, these models represent $\tau$ and not $\nabla \cdot \tau$ this assertion is contingent on the effect that the divergence operator has on the modelling operator.

\footnotetext{
${ }^{2}$ Since a flatter distribution is more likely to be comprised of a larger number of single-phenomenon components of comparable importance.
} 


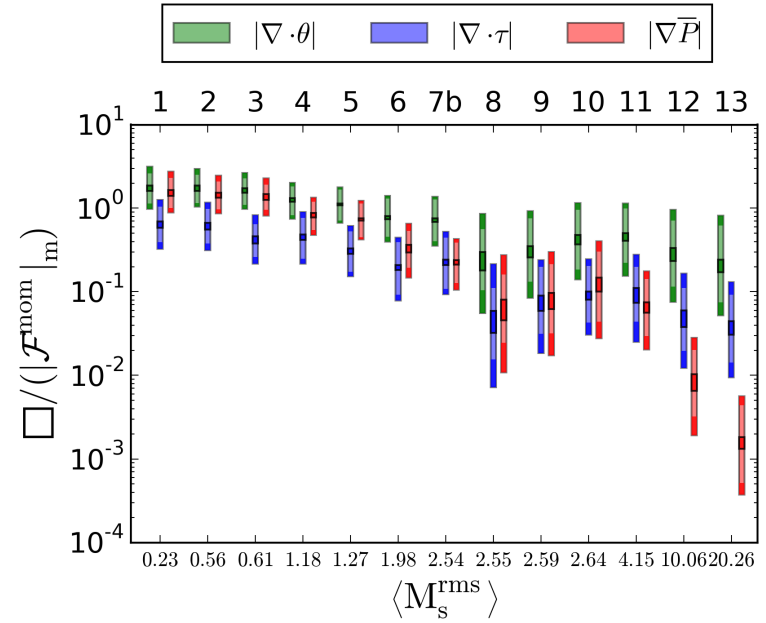

(a)

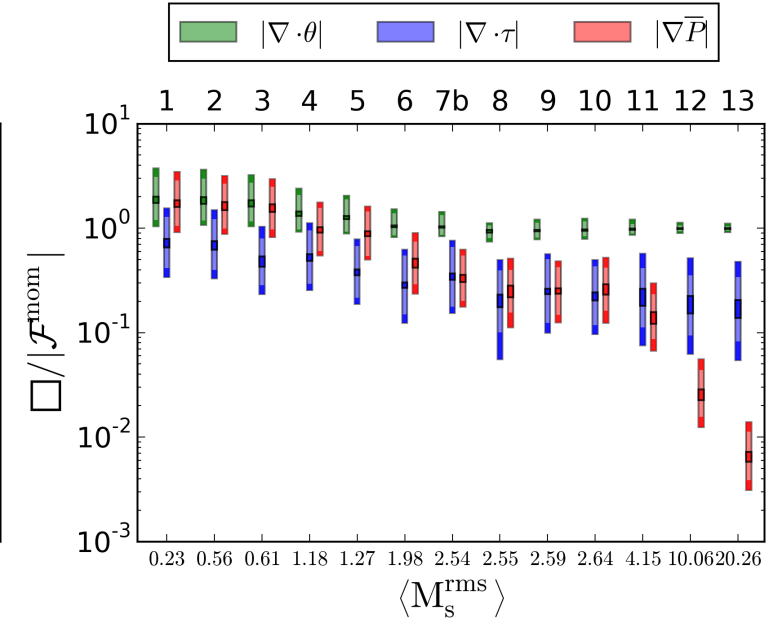

(b)

Figure 3.6: Comparison of $|\nabla \cdot \tau|,|\nabla \cdot \theta|$ and $|\nabla \bar{P}|$ a) globally and b) locally normalised to $\left|\mathcal{F}^{\mathrm{mom}}\right|$ and ordered by average RMS sonic Mach number $\left\langle\mathrm{M}_{\mathrm{s}}^{\mathrm{rms}}\right\rangle$

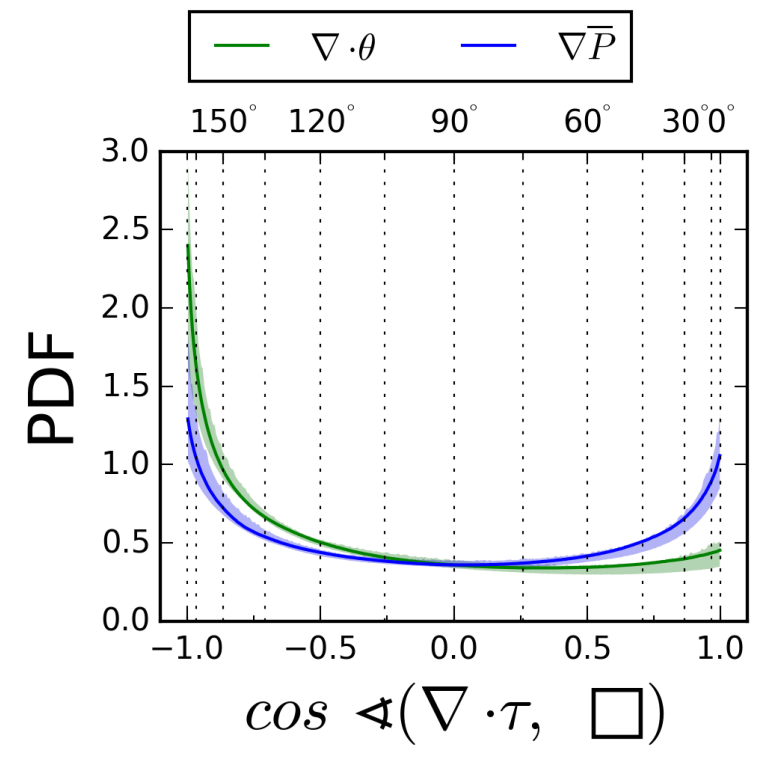

(a) $\operatorname{sim}$. 1-3 (subsonic)

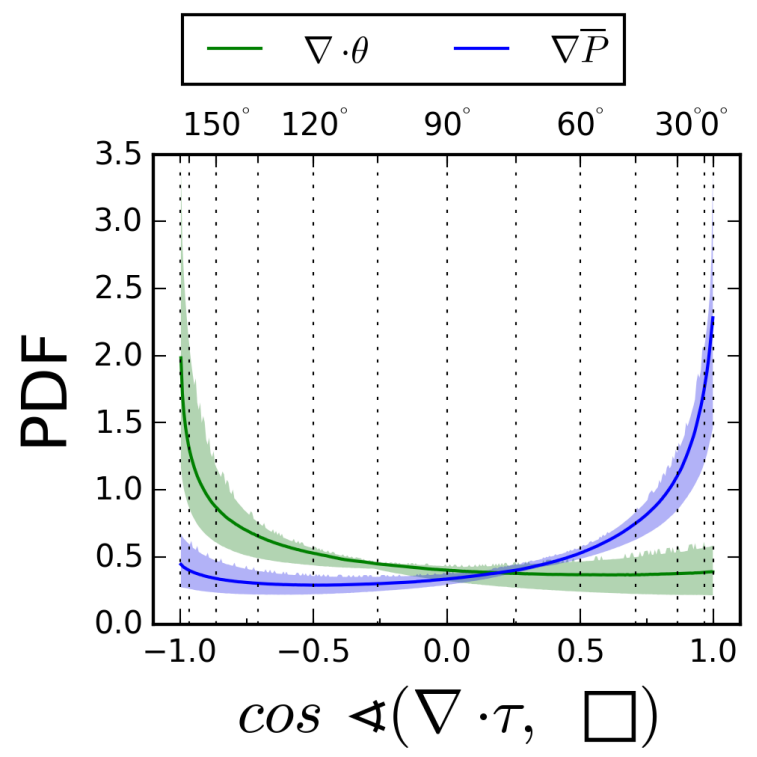

(b) sim. 4-13 (trans/supersonic)

Figure 3.7: Alignment of the total SGS force with the resolved and thermal pressure forces 


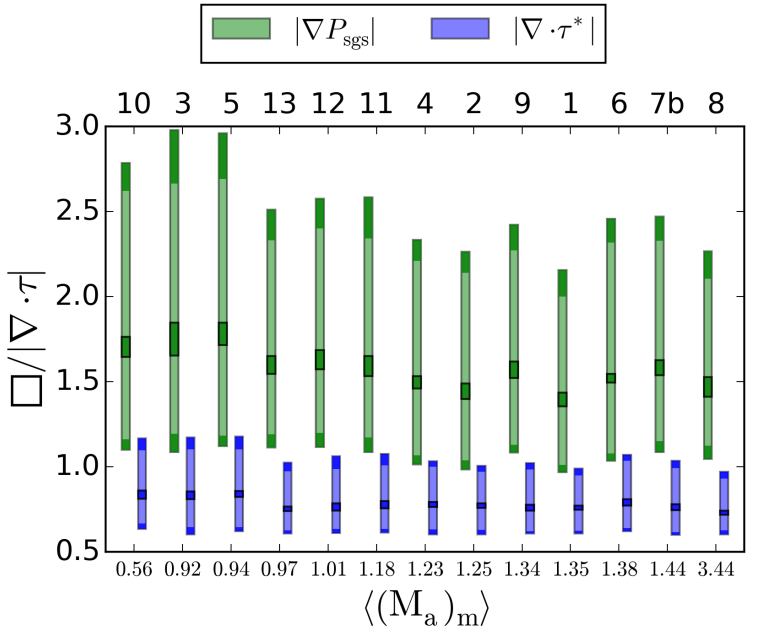

(a)

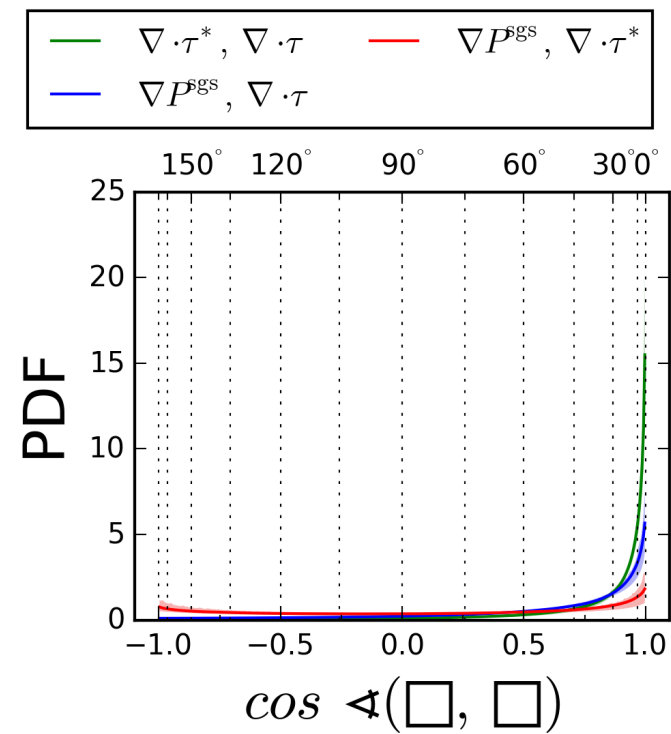

(b)

Figure 3.8: Comparison of the size (a) and orientation (b) of the deviatoric and isotropic parts of the SGS force. The size of the components is normalised locally to the total SGS force $|\nabla \cdot \tau|$.

We can decompose the SGS force further into kinetic and magnetic terms. They are approximately of the same order both in their isotropic and deviatoric components independent of the global Mach number values, (figs. 3.9a and 3.9b). Therefore neglecting one of the two components would not be justified, at least in the range $\left\langle\beta_{\mathrm{p}}\right\rangle \in[0.01,20]$. They also have differently shaped distributions. The kinetic terms have flatter distributions and predominantly positive skewness. Comparing their geometrical orientation (fig. 3.9c), the SGS kinetic and magnetic pressure forces are somewhat aligned while the deviatoric forces are insensitive to each other's orientation. Therefore it will be difficult for one term/one coefficient closures of $\tau^{*}$, like the EV models eq. (2.21), to account for the orientation of the kinetic and magnetic deviatoric SGS forces simultaneously. However, this would be easier for the SGS energy gradients. Thus we expect the EV models to perform well if the SGS orientation and hence anisotropy is not very significant to the modelling and to perform poorly otherwise.

\subsubsection{Tensor analysis of the SGS stress}

So far the discussion was restricted to the SGS forces. The underlying structure however stems from the SGS stress itself. As the SGS stress plays an important part also in the energy and cross-helicity equations, we investigate it directly. We focus on quadratic isometry invariants of $\tau$ and its constituents because they summarise dynamical properties in a frame-independent fashion. As a measure of the magnitude of a second rank tensor we shall use the $L_{2}$ norm given by $\|A\| \equiv \sqrt{A_{i j} A_{j i}}$. We repeat the magnitude comparisons above for the $L_{2}$ norm of the SGS stress. The results are shown 

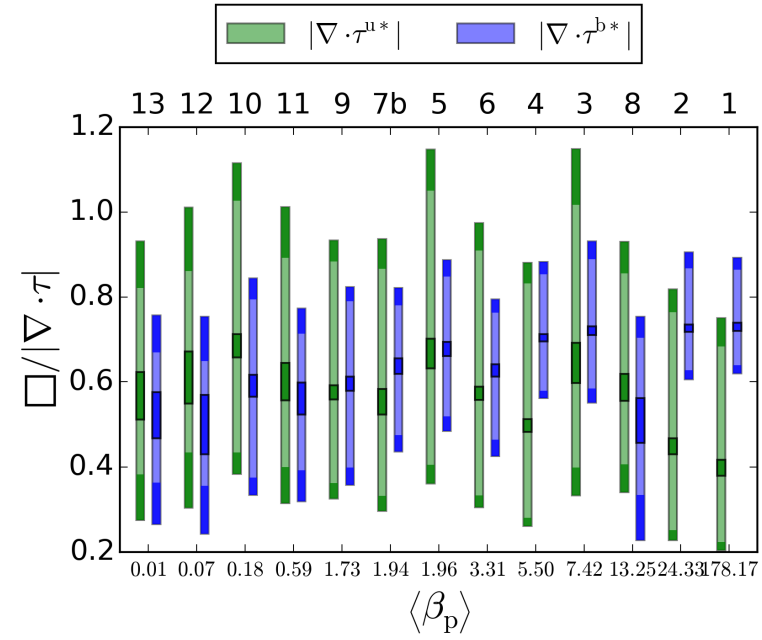

(a)
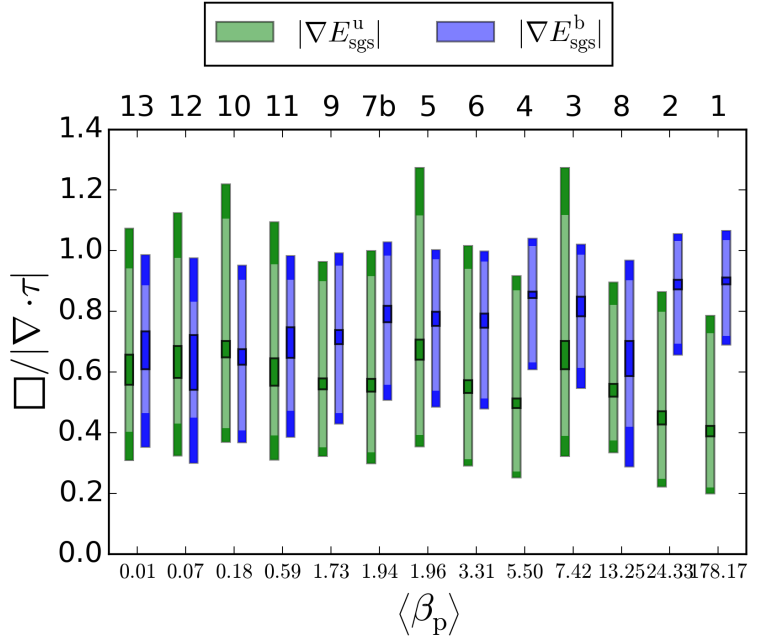

(b)

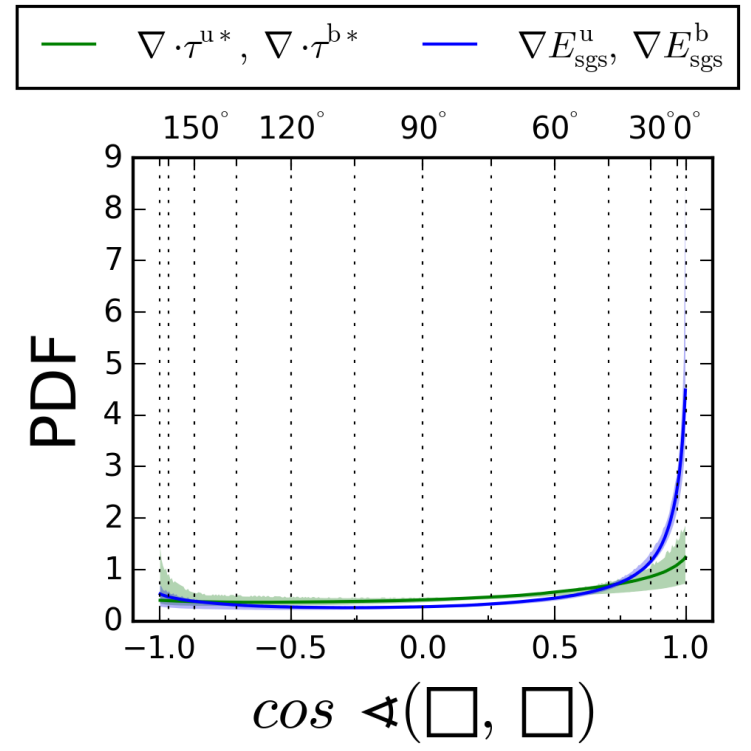

(c)

Figure 3.9: Comparison between the kinetic and magnetic components of the SGS force. The size of the components is normalised locally to the total SGS force $|\nabla \cdot \tau|$. 
in fig. 3.10. The resolved and SGS stresses scale with $\left\langle\mathrm{M}_{\mathrm{s}}{ }^{\mathrm{rms}}\right\rangle$. We note a break in the trend which corresponds to an additional dependence on $\left\langle\beta_{\mathrm{p}}\right\rangle$. The SGS stress is of the order of $10 \%$ to $20 \%$ of the resolved one and follows its dependence on the sonic Mach number. The deviatoric part of $\|\tau\|$ exhibits similar dependence. The SGS pressure is largely independent of the plasma parameters apart from the very low Mach number runs. The deviatoric part of the SGS stress dominates over the SGS pressure for the sonic extremes - sub- and super sonic runs and is still characteristically larger in the trans-sonic runs. It clearly drives the 'size' of the total stress. There doesn't seem to be much difference between the kinetic and magnetic parts of $\tau^{*}$. Based on this alone, it is tempting to model both parts of the SGS stress with the same term. Comparing back to the behaviour of the SGS force fig. 3.9 however, reaffirms the conclusion that the size of the SGS stress, specifically $\tau^{*}$, is a poor characteristic of its function in the momentum equation.

We continue with an investigation of the three primary geometric invariants of the SGS stress. The SGS stress and its components are symmetric second-rank tensors. A general second-rank symmetric tensor $A$ has the following characteristic equation

$$
\lambda^{3}+P \lambda^{2}+Q \lambda+R=0
$$

with eigenvalues $\lambda$. The quantities

$$
\begin{aligned}
P & =-\operatorname{Tr}(A)=-\lambda_{1}-\lambda_{2}-\lambda_{3}, \\
Q & =\frac{1}{2}\left((\operatorname{Tr}(A))^{2}-\operatorname{Tr}\left(A^{2}\right)\right)=\lambda_{1} \lambda_{2}+\lambda_{2} \lambda_{3}+\lambda_{1} \lambda_{3}, \\
R & =-\operatorname{Det}(A)=-\lambda_{1} \lambda_{2} \lambda_{3},
\end{aligned}
$$

are the three primary quadratic invariants of $A$. Note that the $L_{2}$ norm can be expressed as $\|A\|=\sqrt{\operatorname{Tr}\left(A^{\mathrm{T}} A\right)}=P^{2}-2 Q$. The discriminant of the characteristic equation eq. (3.1) is given by

$$
D=P^{2} Q^{2}-4 Q^{3}-4 P^{3} R-27 R^{2}+18 P Q R
$$

We can analyse how the topology of $A$ depends on its geometric invariants (e.g. [46]). For the application of the theory to turbulent hydrodynamics see [20, 21, 64, 82]. Here we provide the basic concepts.

The sign of the discriminant separates the phase space into three regions. In the context of fluid dynamics they have the following topologies:

1. $D<0$ : 1 real and 2 complex-conjugate eigenvalues - vortex topology

2. $D=0$ : 1 or 2 distinct real eigenvalues - axial or isotropic topology

3. $D>0: 3$ real distinct eigenvalues - fully 3-dimensional shear 


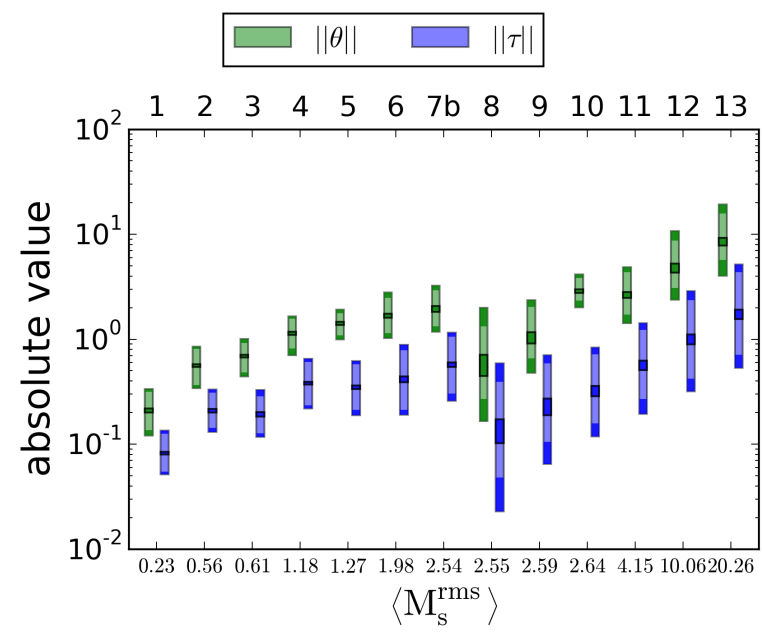

(a)

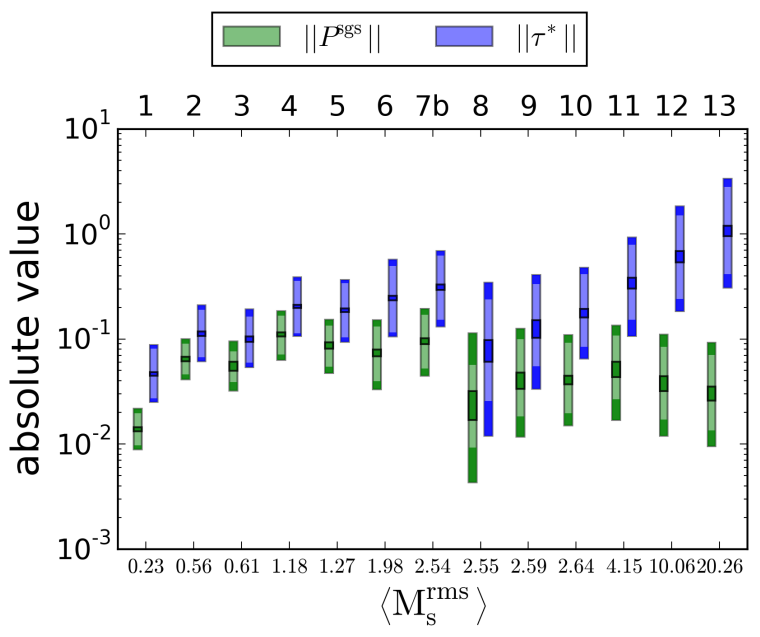

(b)

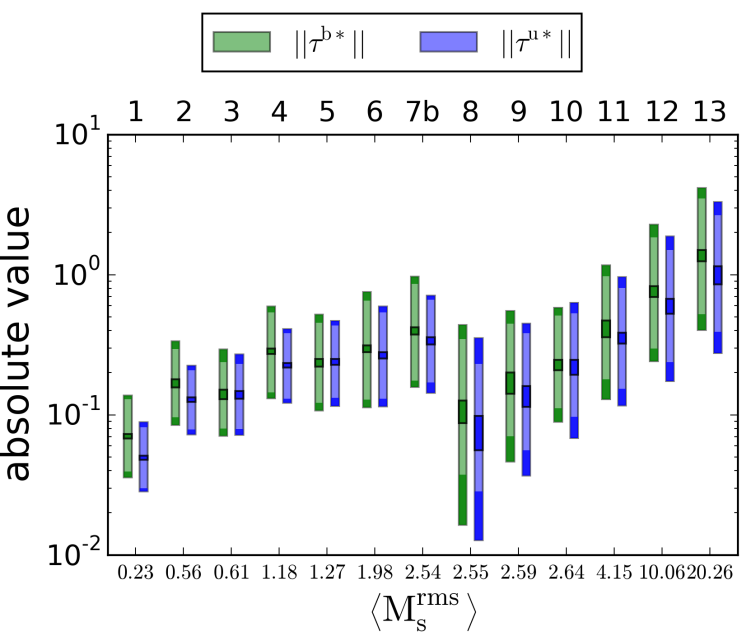

(c)

Figure 3.10: Comparison of the $L_{2}$ norms of a) the SGS and resolved stress, b) the isotropic and deviatoric parts of the SGS stress, and c) the kinetic and magnetic parts of $\tau^{*}$. The values are in code units. 


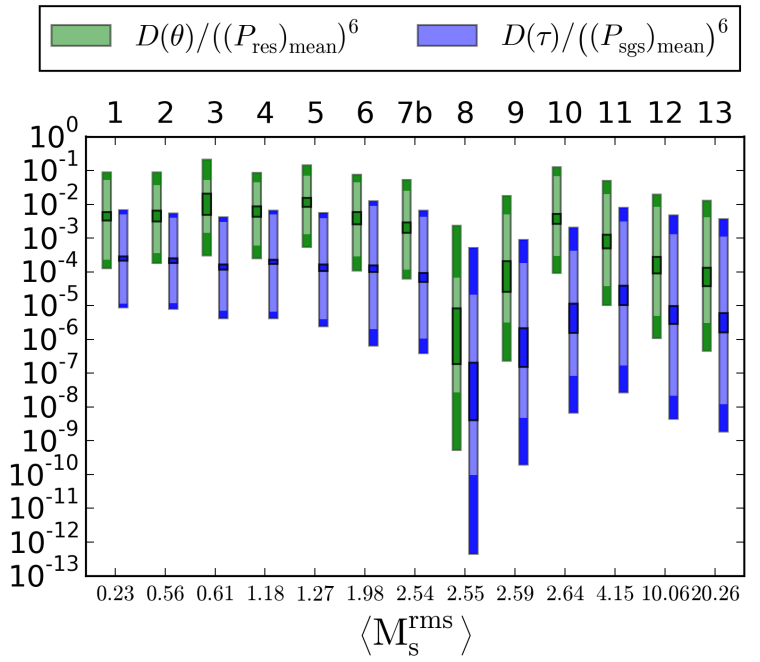

(a)

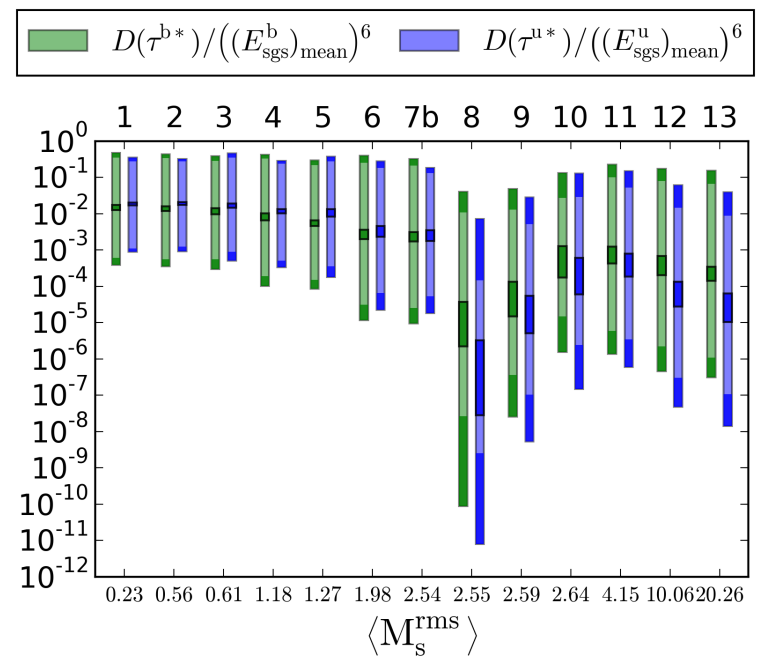

(b)

Figure 3.11: Behaviour of the non-dimensionalized discriminant of the a) SGS and resolved stress and b) kinetic and magnetic SGS deviatoric stress for different simulations. The size of the components is normalised locally to a respective mean pressure. The mean is taken spatially, separately for each snapshot over the simulation domain.

Since all the individual stress terms in the momentum equations are symmetric tensors they always have three real (not necessarily distinct) eigenvalues, in other words they are constrained to have $D \geq 0$. This establishes the general picture of turbulence evolution via shearing and dilatation of eddies. We plot the discriminants of $\theta, \tau, \tau^{\mathrm{u}}$ and $\tau^{\mathrm{b}}$ in fig. 3.11. Although the discriminant value does not distinguish between axisymmetric and isotropic topologies, the smaller it is, the more degenerate the eigenvalues are. In order to gauge the dynamical importance of the degeneracy effect, we rescale the discriminants with the spatially averaged dynamical pressure (resolved or SGS, as appropriate), i.e. the mean isotropic deformation. For the kinetic and magnetic components the role of pressure is taken by the respective SGS energy. Overall, we see that the SGS and resolved normalised discriminants are quite small, which is expected for the case of isotropic turbulence. The resolved stress exhibits larger anisotropy than the SGS one. Since the forcing takes place at resolved scales this confirms that the isotropy is a property of the intrinsic flow dynamics and not the forcing. The discriminants of the total deviatoric stresses $\theta^{*}$ and $\tau^{*}$ (not shown) exhibit the same qualitative behaviour. This is expected since they differ from $\theta$ and $\tau$ only by an isotropic component. Therefore we can simplify the discussion by considering only the deviatoric terms.

Individually the kinetic and magnetic deviatoric SGS components exhibit much higher degree of anisotropy than the total SGS stress. Therefore there must be a coherence between $\tau^{\mathrm{u} *}$ and $\tau^{\mathrm{b} *}$ which leads to a high degree of cancellation between their anisotropic effects. Note that this is stronger for lower sonic Mach numbers (simulations 1-10) than for higher ones.

Combining the signs of the discriminant and the determinant we can identify four 
regions with different topologies. Since we deal with a positive discriminant, only two of them are accessible. For negative determinant, $R>0$, we have either sheet topology, $\lambda_{1} \geq \lambda_{2}>0>\lambda_{3}$, i.e expansion along two eigenvectors and compression along the third, or a stable node $0>\lambda_{1} \geq \lambda_{2} \geq \lambda_{3}$, i.e. total compression. If the determinant is positive $R<0$, we have either tube topology, $\lambda_{1}>0 \geq \lambda_{2} \geq \lambda_{3}-$ expansion along one eigenvector and compression along the other two, or an unstable node $\lambda_{1} \geq \lambda_{2} \geq \lambda_{3}=0$, i.e. total expansion. This is summarized in table 3.1. The remaining freedom is prescribed by a third invariant, e.g. the trace. Since the traceless components of the stresses carry, trivially, the same anisotropy information about the discriminant as the total stress, the discussion is confined to the traceless tensors. Then the discriminant simplifies to $D^{*}=-4 Q^{3}-27 R^{2}$ and the phase space simplifies to the tube or sheet topologies.

\begin{tabular}{c|c|c|}
$D \geq 0$ & $P \neq 0$ & $P=0$ \\
\hline$R<0$ & tube or unstable node & tube \\
& $\lambda_{1}>0>=\lambda_{2}>=\lambda_{3}$ or $\lambda_{1} \geq \lambda_{2} \geq \lambda_{3}=0$ & $\lambda_{1}>0>\lambda_{2}>\lambda_{3}$ \\
\hline$R>0$ & sheet or stable node & sheet \\
& $\lambda_{1}>=\lambda_{2}>0>\lambda_{3}$ or $0>\lambda_{1} \geq \lambda_{2} \geq \lambda_{3}$ & $\lambda_{1}>\lambda_{2}>0>\lambda_{3}$ \\
\hline
\end{tabular}

Table 3.1: Topology regions of a second rank symmetric tensor with trace $P$, determinant $-R$ and discriminant $D$.

To analyse the topology of the data (following [24]) we consider the joint histogram of $Q$ versus $R$. The curve $D=0$ provides an upper boundary for the data and the determinant distinguishes the different regions. In fig. 3.12 we show a sample snapshot for $\tau^{\mathrm{u} *}, \tau^{\mathrm{b} *}, \tau^{*}$, and $\theta^{*}$. We see that there is preferentially tube structure in both the kinetic and magnetic components of $\tau^{*}$, but when combined they can produce sheet and tube topology with comparable frequency. Intuitively, this can occur in the simplest case when the kinetic eigenvector associated with the positive eigenvalue is aligned with a magnetic eigenvector associated with a smaller (in magnitude) negative eigenvalue and vice versa. In that case these two eigenvectors will span the topological sheet of total SGS stress, while the remaining kinetic and magnetic eigenvectors are orthogonal to it (and work to compress it). The total resolved stress favours both topologies with similar strength, however the shape of the distribution is qualitatively different from the total SGS one. Clearly, the determinant is a key characteristic that distinguishes between the kinetic, magnetic and total SGS scales, but also perhaps between SGS and resolved one.

Collecting the points with tube and sheet topologies from each snapshot and summarising the results for all simulations we reach fig. 3.13. The behaviour of $\tau^{\mathrm{u} *}$ and $\tau^{\mathrm{b} *}$ observed in fig. 3.12 appears quite robust and may be used as a diagnostic criterion for SGS closures. In approximately $90 \%$ of the cells in the simulation domain these tensors induce tube topology, with two negative and one positive eigenvalue. The remaining $\sim 10 \%$ exhibit sheet topology with two positive and one negative eigenvalue. The total deviatoric SGS stress shows more variation with time and plasma parameters. For low 

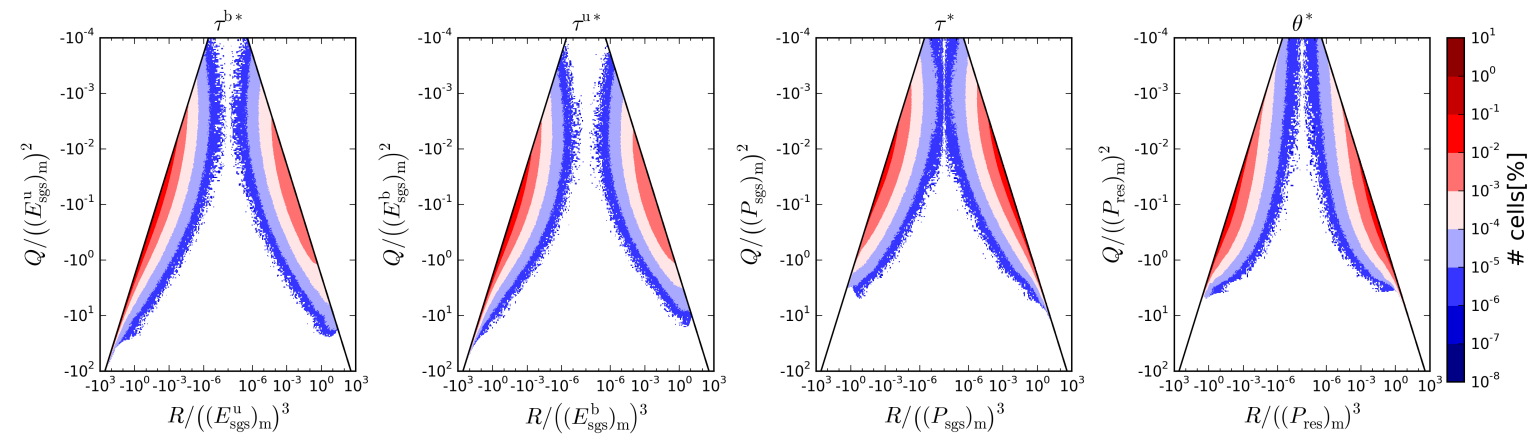

Figure 3.12: Sample snapshot: joint histogram of the $Q$ and $R$ invariants for the deviatoric stresses $\tau^{*}, \tau^{\mathrm{u} *}, \tau^{\mathrm{b} *}, \theta^{*}$, normalised by a spatially average appropriate pressure term. The black line follows the $D^{*}=0$ boundary.
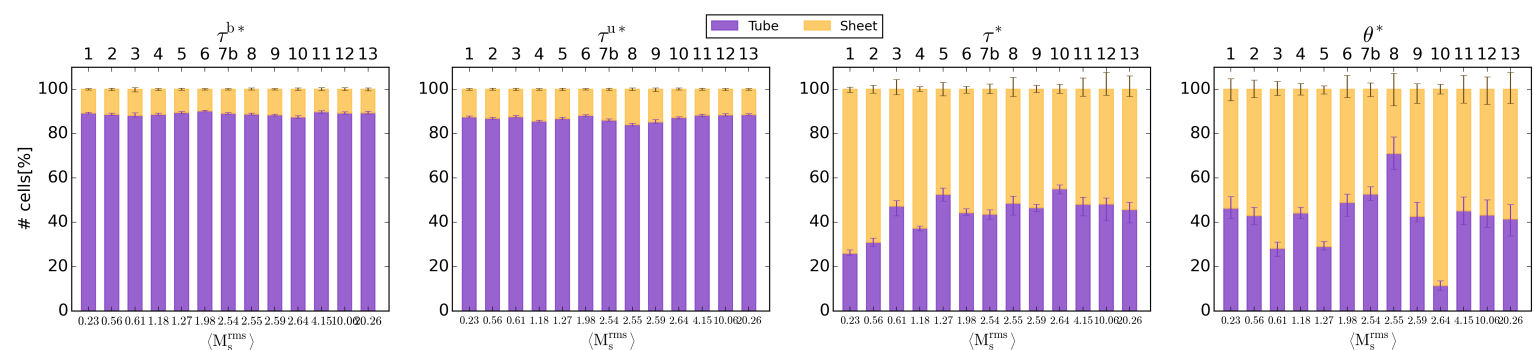

Figure 3.13: Summary of the topology of the deviatoric stresses $\tau^{*}, \tau^{\mathrm{u} *}, \tau^{\mathrm{b} *}, \theta^{*}$ within all simulations. Error bars at the top of each topological region represent variations within the time interval between $2 T$ and $5 T$

sonic Mach numbers the dominant contribution comes from the sheet topology. In the trans and super-sonic regime this decreases to approximate equipartition between the two topologies. No strong trend is observed for $\theta^{*}$, neither is there an obvious relation between the distributions of $\tau^{*}$ and $\theta^{*}$. One possible explanation can be that the topology of the resolved stress is more strongly influenced by the stochastic nature of the forcing, which dominates the dynamics of the super-filter scales. Note that this influence is not trivially related to the compressive-to-solenoidal ratio - in simulations 8,9 and 10 this ratio is 0.9 , while in all other simulations it is 0.5 . Alternatively, the resolved stress topology may depend more strongly on the mean plasma parameters that the SGS stress one.

More data is necessary to make a conclusive statement and to determine whether this behaviour extends to other initial and boundary conditions and to decaying turbulence. It should be noted that in hydrodynamics the behaviour of the resolved flow follows strongly and robustly the $D \sim 0$ regions, known as Vieilliefosse tails [96], and is similar to the distribution of $\tau^{*}$. However, in contrast to the data examined here, there the vortical regions $D<0$ are well represented as well. 


\subsubsection{SGS pressure}

We conclude the discussion of the SGS stress with an investigation of its isotropic part, the SGS pressure. Mainly we focus on the approximation from section 2.3.1 to neglect (or close implicitly) the turbulence pressure in favour of the thermal one ${ }^{3}$.

Let us denote the resolved dynamic pressure as $P_{\mathrm{res}}=2 E_{\mathrm{res}}^{\mathrm{u}}+E_{\mathrm{res}}^{\mathrm{b}}$. Then the total MHD pressure is given by $P_{\text {tot }}=P_{\text {res }}+P_{\text {sgs }}+\bar{P}$. Figure 3.14 shows the distributuion of the local and global contributions of the pressure components to the total pressure as well as the local ratio $P_{\mathrm{sgs}} / P_{\text {res }}$. We observe the same scaling with sonic Mach number in the local and global plots. The SGS pressure maintains a steady contribution of the order of $10 \% P_{\text {tot }}$ except in the most incompressible simulation, while $P_{\text {res }}$ and $\bar{P}$ exhibit strong scaling with $\left\langle\mathrm{M}_{\mathrm{s}}^{\mathrm{rms}}\right\rangle$. Figure 3.14b demonstrates that the SGS pressure dominates over the thermal one when the RMS sonic Mach number exceeds $\sim 10$ and remains dynamically important down to at least $\left\langle\mathrm{M}_{\mathrm{s}}{ }^{\mathrm{rms}}\right\rangle \sim 1.9$. Interestingly, there seems to be a dependence on $\left\langle\mathrm{M}_{\mathrm{S}}{ }^{\mathrm{rms}}\right\rangle$ of the relative importance of the SGS to resolved pressure. With increasing sonic Mach number in the supersonic regime a larger part of the dynamic pressure is resolved.

Consider the impact of the pressure on the momentum equation, as shown in fig. 3.15. The SGS pressure force $\left|\nabla P_{\text {sgs }}\right|$ decreases slightly with increasing $\left\langle\mathrm{M}_{\mathrm{s}}{ }^{\mathrm{rms}}\right\rangle$ similarly to $|\nabla \cdot \tau|$. It is about 10 times more important than the SGS pressure itself. Just as in the induction equation, this is unsurprising since stronger gradients at smaller scales are expected. However, the SGS pressure force is of the order and can exceed the size of the local and the average total momentum flux itself. Comparing the local contributions with respect to the total pressure force, we see that the resolved pressure gradient dominates, especially at the high Mach numbers. The SGS pressure gradient, however, is in all simulations at least of the same size as the thermal pressure gradient.

Considering the anisotropy of the SGS pressure force, we already established a weak alignment between the SGS kinetic and magnetic components in fig. 3.9c. Figure 3.16a shows the alignment of these components with their resolved counterparts. There appears to be moderate collinearity with significant variation over time and plasma parameters. The alignment with the thermal pressure gradient is somewhat better, fig. 3.16b, but also suffers from large variations.

To summarise, the SGS pressure in its own right, as well as its contribution to the momentum equation clearly has at least as strong an impact on the dynamics as the thermal pressure and should be modelled with care. Combining the two terms would be ill-advised considering their variable alignment. Additionally, due to their different physical origins they would give rise to radically different equations of state, have different statistical distributions and evolutionary time-scales. We conclude that at least in isothermal homogeneous and isotropic MHD turbulence the SGS pressure should not be ignored or modelled implicitly, neither within the numerical scheme nor as an augmentation of the thermal pressure.

\footnotetext{
${ }^{3}$ Considering the nearly isothermal conditions of our simulations for the remainder of this chapter we have simply used $\bar{P}=c_{s}^{2} \bar{\rho}$, with sound speed $c_{s}=1$.
} 


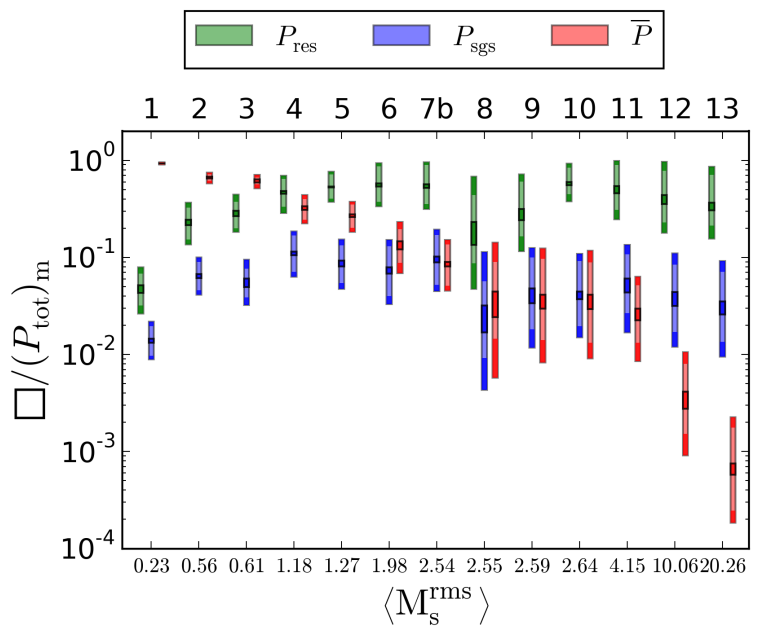

(a)
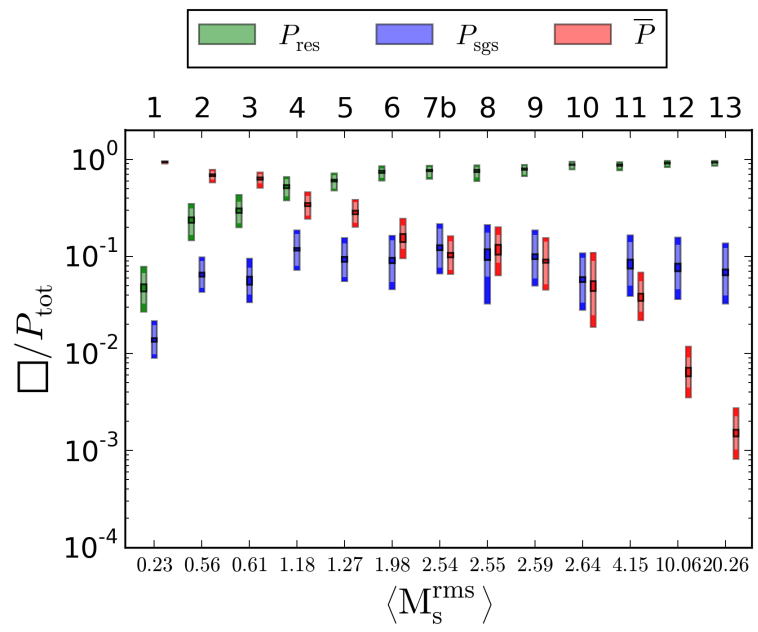

(b)

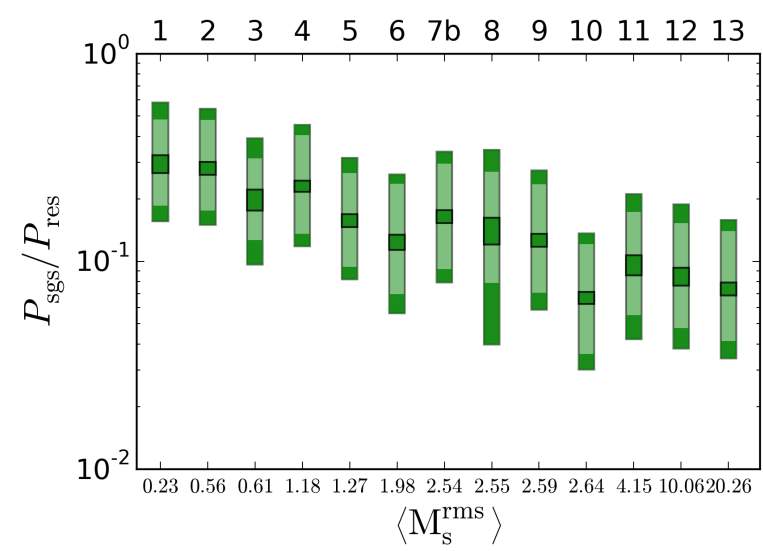

(c)

Figure 3.14: Relative strength of $\bar{P}, P^{\mathrm{sgs}}$ and $P^{\mathrm{res}}$, normalised a) to the mean total pressure and b) to the local total pressure. Panel c) shows the relative strength of the SGS to resolved pressure. 


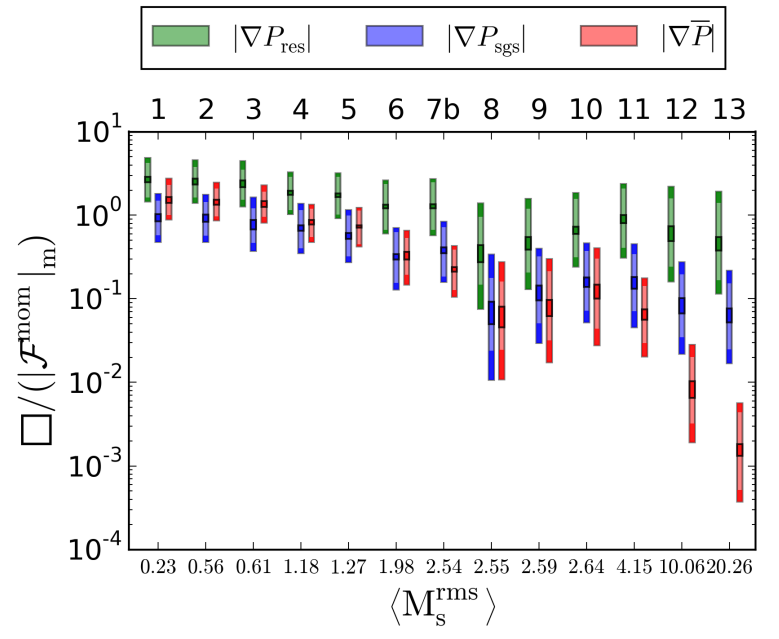

(a)

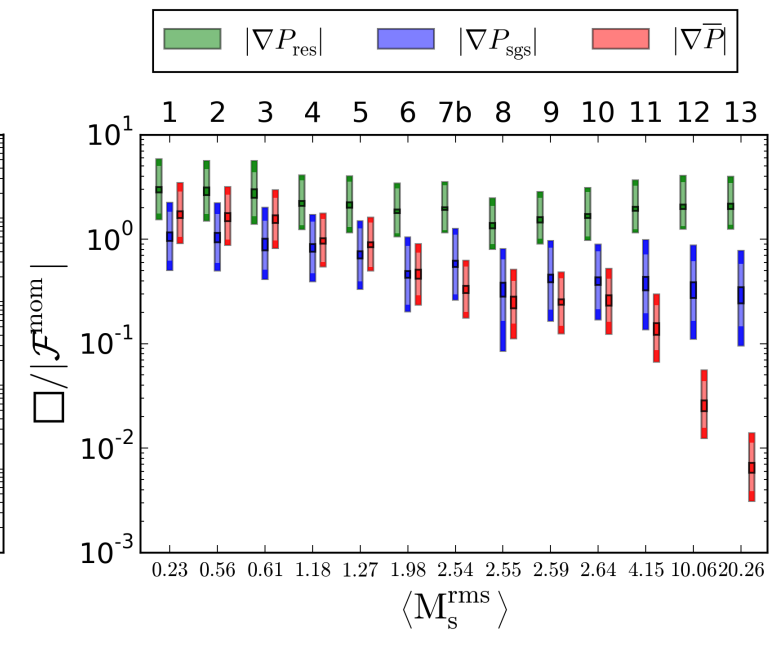

(b)

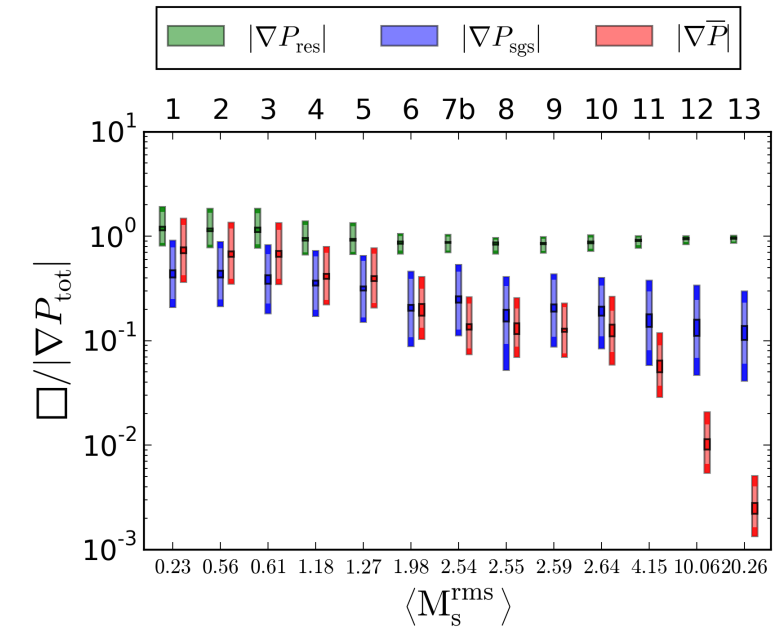

(c)

Figure 3.15: Relative strength of the pressure forces $|\nabla \bar{P}|,\left|\nabla P^{\mathrm{res}}\right|$ and $\left|\nabla P^{\mathrm{sgs}}\right|$, normalised to a) the average magnitude of the total momentum flux, b) the local magnitude of the total momentum flux and c) the local magnitude of the total pressure force. 


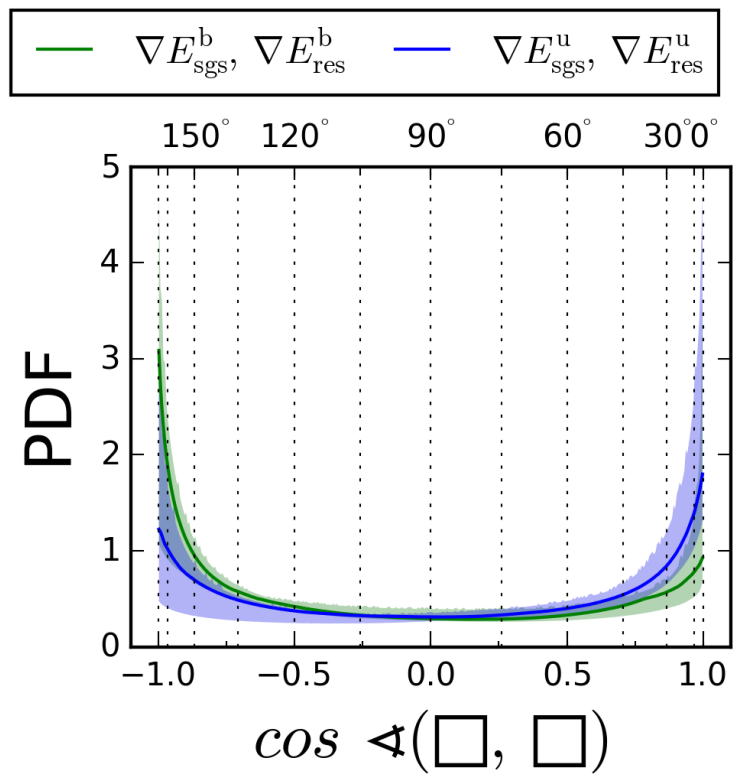

(a)

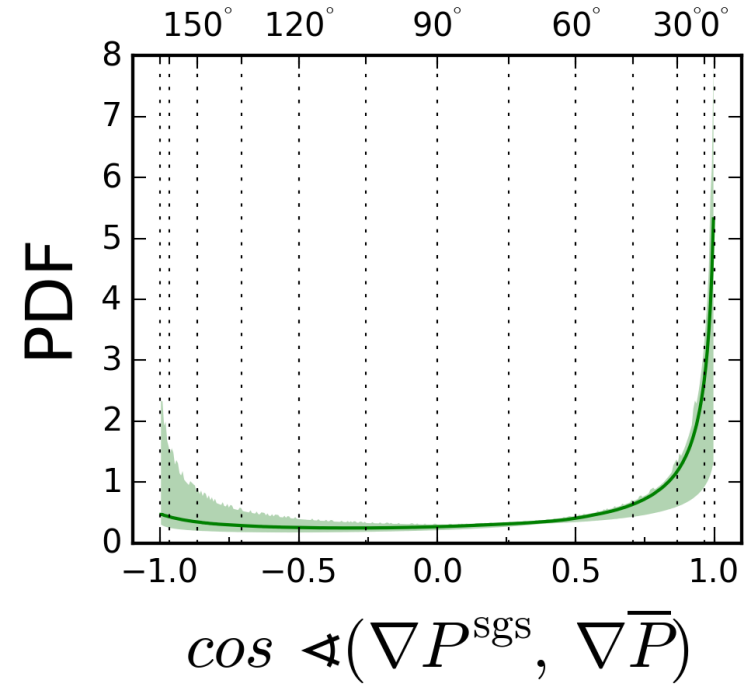

(b)

Figure 3.16: Alignment between a) the SGS and resolved parts of the kinetic and magnetic pressure forces (energy gradients), b) the total SGS and thermal pressure forces.

\subsection{Energy equations}

As shown in chapter 2 the SGS energy plays a central role in most of the described SGS closures. Furthermore, the resolved energy is the main characteristic of the flow, to which we have direct access in LES. In this section we examine their evolution equations and comment on the model-building hypotheses used previously. Specifically, we shall examine the assumption of local balance, which leads to the expressions of the Smagorinsky energies.

First, let us identify the key points of the energy dynamics gleaned from eqs. (1.22), (1.23), (1.25)-(1.28) and (1.30)-(1.32). The resolved kinetic energy decays through the flux term $\mathcal{F}_{2}^{\mathrm{u}}=\tilde{\mathbf{u}} \cdot(\nabla \cdot \tau)$ into SGS energy. Similarly, the resolved magnetic energy decays via $\mathcal{F}_{2}^{\mathrm{b}}=-\overline{\mathbf{B}} \cdot \nabla \times \mathcal{E}$. Note that these are the only SGS terms in the resolved equations. For consistency with the SGS energy equations and to denote the order of the SGS terms they contain, they are denoted the subscript 2. We can separate the terms into transport (or diffusion) $\mathcal{T}$ and cascade $\Sigma$ components. We obtain

$$
\begin{aligned}
& \mathcal{F}_{2}^{\mathrm{u}}=\mathcal{T}_{2}^{\mathrm{u}}+\Sigma_{2}^{\mathrm{u}}=\nabla \cdot(\tilde{\mathbf{u}} \cdot \tau)-\tau \cdot \tilde{\mathcal{S}} \\
& \mathcal{F}_{2}^{\mathrm{b}}=\mathcal{T}_{2}^{\mathrm{b}}+\Sigma_{2}^{\mathrm{b}}=\nabla \cdot(\overline{\mathbf{B}} \times \mathcal{E})-\mathcal{E} \cdot \overline{\mathbf{J}}
\end{aligned}
$$

The total transfer between SGS and resolved energy is simply the sum of the kinetic 
and magnetic contributions. We denote

$$
\begin{aligned}
& \mathcal{F}_{2}^{\mathrm{en}}=\mathcal{F}_{2}^{\mathrm{u}}+\mathcal{F}_{2}^{\mathrm{b}}, \\
& \mathcal{T}_{2}^{\mathrm{en}}=\mathcal{T}^{\mathrm{u}}+\mathcal{T}^{\mathrm{b}}=\nabla \cdot(\tilde{\mathbf{u}} \cdot \tau+\overline{\mathbf{B}} \times \mathcal{E}), \\
& \Sigma_{2}^{\mathrm{en}}=\Sigma_{2}^{\mathrm{u}}+\Sigma_{2}^{\mathrm{b}}=-\tau \cdot \tilde{\mathcal{S}}-\mathcal{E} \cdot \overline{\mathbf{J}} .
\end{aligned}
$$

With these the definitions the resolved energy equations take the form

$$
\begin{aligned}
& \frac{\partial}{\partial t} E_{\mathrm{res}}^{\mathrm{u}}+\nabla \cdot\left(\tilde{\mathbf{u}} E_{\mathrm{res}}^{\mathrm{u}}\right)+\tilde{\mathbf{u}} \times \overline{\mathbf{B}} \cdot \overline{\mathbf{J}}+\tilde{\mathbf{u}} \cdot \nabla \bar{P}+\mathcal{T}_{2}^{\mathrm{u}}=-\Sigma_{2}^{\mathrm{u}}+\epsilon_{\mathrm{res}}^{\mathrm{u}}, \\
& \frac{\partial}{\partial t} E_{\mathrm{res}}^{\mathrm{b}}+\nabla \cdot(\overline{\mathbf{B}} \times(\tilde{\mathbf{u}} \times \overline{\mathbf{B}}))-\tilde{\mathbf{u}} \times \overline{\mathbf{B}} \cdot \overline{\mathbf{J}}+\mathcal{T}_{2}^{\mathrm{b}}=-\Sigma_{2}^{\mathrm{b}}+\epsilon_{\mathrm{res}}^{\mathrm{b}}, \\
& \frac{\partial E_{\mathrm{res}}}{\partial t}+\nabla \cdot\left(\tilde{\mathbf{u}} E_{\mathrm{res}}^{\mathrm{u}}+2 \tilde{\mathbf{u}} E_{\mathrm{res}}^{\mathrm{b}}-\overline{\mathbf{B}} H_{\mathrm{res}}\right)+\tilde{\mathbf{u}} \cdot \nabla \bar{P}+\mathcal{T}_{2}^{\mathrm{en}}=-\Sigma_{2}^{\mathrm{en}}+\epsilon_{\mathrm{res}}^{\mathrm{en}}
\end{aligned}
$$

In this form it is clear that the transfer between resolved kinetic and magnetic energies happens through the term $\tilde{\mathbf{u}} \times \overline{\mathbf{B}} \cdot \overline{\mathbf{J}}$. We have also used the identity for the Poynting flux $\mathbf{B} \times(\mathbf{u} \times \mathbf{B}) \equiv 2 \mathbf{u} E^{\mathrm{b}}-\mathbf{B} H$ to demonstrate one of the roles of cross-helicity $H$ in the energy dynamics - it counteracts the diffusion of magnetic energy ${ }^{4}$. This adds motivation to the study of the SGS cross-helicity equation in the following section. Completing the set of energies, the SGS energy equations are given by

$$
\begin{aligned}
\frac{\partial}{\partial t} E_{\mathrm{sgs}}^{\mathrm{u}}+\mathcal{F}_{3}^{\mathrm{u}}+\mathcal{F}^{\mathrm{en}, \text { ther }}-\mathcal{T}_{2}^{\mathrm{u}} & =\Sigma_{2}^{\mathrm{u}}+\epsilon_{\mathrm{sgs}}^{\mathrm{u}}, \\
\frac{\partial}{\partial t} E_{\mathrm{sgs}}^{\mathrm{b}}+\mathcal{F}_{3}^{\mathrm{b}}-\mathcal{T}_{2}^{\mathrm{b}} & =\Sigma_{2}^{\mathrm{b}}+\epsilon_{\mathrm{sgs}}^{\mathrm{b}}, \\
\frac{\partial}{\partial t} E_{\mathrm{sgs}}+\mathcal{F}_{3}^{\mathrm{en}}+\mathcal{F}^{\mathrm{en}, \text { ther }}-\mathcal{T}_{2}^{\mathrm{en}} & =\Sigma_{2}^{\mathrm{en}}+\epsilon_{\mathrm{sgs}}^{\mathrm{en}} .
\end{aligned}
$$

$\mathcal{F}_{3}^{\square}$ denotes third order SGS terms. We give their explicit form along with the contribution from the thermal pressure term:

$$
\begin{aligned}
\mathcal{F}_{3}^{\mathrm{u}} & =\mathcal{T}_{3}^{\mathrm{u}}+\Sigma_{3}^{\mathrm{u}}, \\
\mathcal{T}_{3}^{\mathrm{u}} & =\nabla \cdot\left(\overline{\mathbf{u} E^{\mathrm{u}}}-\tilde{\mathbf{u}} E_{\mathrm{res}}^{\mathrm{u}}\right) \\
\Sigma_{3}^{\mathrm{u}} & =\overline{\mathbf{u} \cdot \mathbf{B} \times \mathbf{J}}-\tilde{\mathbf{u}} \cdot \overline{\mathbf{B}} \times \overline{\mathbf{J}} \\
\mathcal{F}^{\mathrm{en}, \text { ther }} & =\overline{\mathbf{u} \cdot \nabla(P)}-\tilde{\mathbf{u}} \cdot \nabla(\bar{P}), \\
\mathcal{F}_{3}^{\mathrm{b}}=\mathcal{T}_{3}^{\mathrm{b}}+\Sigma_{3}^{\mathrm{b}} & =\overline{\mathbf{B} \cdot \nabla \times(\mathbf{u} \times \mathbf{B})}-\overline{\mathbf{B}} \cdot \nabla \times(\tilde{\mathbf{u}} \times \overline{\mathbf{B}}), \\
\mathcal{T}_{3}^{\mathrm{b}} & =\nabla \cdot(\overline{\mathbf{B} \times(\mathbf{u} \times \mathbf{B})}-\overline{\mathbf{B}} \times(\tilde{\mathbf{u}} \times \overline{\mathbf{B}})), \\
\Sigma_{3}^{\mathrm{b}} & =-(\overline{\mathbf{u} \times \mathbf{B} \cdot \mathbf{J}}-\tilde{\mathbf{u}} \times \overline{\mathbf{B}} \cdot \overline{\mathbf{J}}), \\
\mathcal{F}_{3}^{\mathrm{en}}=\mathcal{T}_{3}^{\mathrm{en}} & =\mathcal{T}_{3}^{\mathrm{u}}+\mathcal{T}_{3}^{\mathrm{b}}, \\
\Sigma_{3}^{\mathrm{en}} & =0 .
\end{aligned}
$$

${ }^{4}$ The other role is the kinetic to magnetic energy transfer, incorporated in $\tilde{\mathbf{u}} \times \overline{\mathbf{B}} \cdot \overline{\mathbf{J}}$. 
Since we don't have direct access to the energy dissipation rates from our data, we omit them from the discussion. This also prohibits the investigation of the internal energy equation directly. Consequently, and for simplicity, we treat the thermal pressure flux $\mathcal{F}^{\text {en,ther }}$ as a whole without separating it into a transport and cascade components. We shall further adopt the notation for the total flux, transport and cascade terms:

$$
\begin{gathered}
\mathcal{F}^{\mathrm{u}}=\mathcal{F}_{2}^{\mathrm{u}}-\mathcal{F}_{3}^{\mathrm{u}}-\mathcal{F}^{\mathrm{en}, \text { ther }}, \\
\mathcal{F}^{\mathrm{en}}=\mathcal{F}_{2}^{\mathrm{en}}-\mathcal{F}_{3}^{\mathrm{en}}-\mathcal{F}^{\mathrm{en}, \text { ther }} \\
\mathcal{T}^{X}=\mathcal{T}_{2}^{X}-\mathcal{T}_{3}^{X} \text { and } \Sigma^{X}=\Sigma_{2}^{X}-\Sigma_{3}^{X} \text { for } X \in\{\mathrm{u}, \mathrm{b}, \text { en }\} .
\end{gathered}
$$

It should be noted that the separation into diffusion and cascade terms is not unique. It is done in the form of eqs. (3.4) and (3.5) to allow the treatment of the kinetic and magnetic parts of the SGS stress in an analogous manner. This is motivated by the appearance of both $\tau^{\mathrm{u}}$ and $\tau^{\mathrm{b}}$ under a divergence operator in the momentum equation. Furthermore, in this form there is an apparent symmetry between the terms $\Sigma_{2}^{u}$ and $\Sigma_{2}^{\mathrm{b}}$ - they both contain derivatives of resolved fields and second order SGS terms. As we saw from the momentum and induction equations, modelling the SGS terms and their derivatives are two quite different problems. Thus, because we are concerned with models for the SGS terms themselves and not their derivatives, we adhere to this form of the separation.

For completeness we demonstrate an alternative formulation. It can be obtained analytically by decomposing the evolution equation for the total filtered energy into kinetic and magnetic components. Allowing for the symmetry of the vector triple product, there is a correspondence between the quantities

$$
\tilde{\mathbf{u}} \cdot\left(\nabla \cdot \tau^{\mathrm{b}}\right)=-\tilde{\mathbf{u}} \cdot \overline{\mathbf{B} \times \mathbf{J}}+\tilde{\mathbf{u}} \cdot \overline{\mathbf{B}} \times \overline{\mathbf{J}}
$$

and

$$
\mathcal{E} \cdot \overline{\mathbf{J}}=\overline{\mathbf{u} \times \mathbf{B}} \cdot \overline{\mathbf{J}}-\tilde{\mathbf{u}} \times \overline{\mathbf{B}} \cdot \overline{\mathbf{J}} .
$$

With this in mind we can recast the total SGS energy equation in the form

$$
\begin{aligned}
& \frac{\partial}{\partial t} E_{\mathrm{sgs}}+ \\
& \nabla \cdot\left(\overline{\mathbf{u} E}-\tau^{\mathrm{u}} \cdot \tilde{\mathbf{u}}-\tilde{\mathbf{u}} E_{\mathrm{res}}^{\mathrm{u}}+\overline{\mathbf{B} \times(\mathbf{u} \times \mathbf{B})}-\overline{\mathbf{B}} \times \mathcal{E}-\overline{\mathbf{B}} \times(\tilde{\mathbf{u}} \times \overline{\mathbf{B}})\right)+ \\
& \tau^{\mathrm{u}} \cdot \tilde{\mathcal{S}}+\underbrace{\left(\overline{\mathbf{u} \times \mathbf{B} \cdot \overline{\mathbf{J}}+\tilde{\mathbf{u}} \cdot \overline{\mathbf{B} \times \mathbf{J}})}=\epsilon_{\mathrm{sgs}}^{\mathrm{en}} \cdot\right.}_{\tilde{\mathbf{u}} \cdot\left(\nabla \cdot \tau^{\mathrm{b}}\right): \text { cascade }}
\end{aligned}
$$

In this form the term $\nabla \cdot\left(\tau^{\mathrm{b}} \cdot \tilde{\mathcal{S}}\right)$ is simply reinterpreted as part of the magnetic energy cascade, in other words the entire term $\tilde{\mathbf{u}} \cdot\left(\nabla \cdot \tau^{\mathrm{b}}\right)$, denoted by the under-brace in the equation above, is considered a cascade term. There is no explicit transport (diffusion) of SGS kinetic energy by the magnetic agents. 
Compare this to our formulation, to which we arrived synthetically by combining the SGS kinetic and magnetic energy equations,

$$
\begin{aligned}
& \frac{\partial}{\partial t} E_{\mathrm{sgs}}+ \\
& \nabla \cdot(\overline{\mathbf{u} E}-\tau^{\mathrm{u}} \cdot \tilde{\mathbf{u}}-\tilde{\mathbf{u}} E_{\mathrm{res}}^{\mathrm{u}}+\underbrace{\tau^{\mathrm{b}} \cdot \tilde{\mathbf{u}}}_{\text {transport }}+\overline{\mathbf{B} \times(\mathbf{u} \times \mathbf{B})}-\overline{\mathbf{B}} \times \mathcal{E}-\overline{\mathbf{B}} \times(\tilde{\mathbf{u}} \times \overline{\mathbf{B}}))+ \\
& \quad \tau \cdot \tilde{\mathcal{S}}+\underbrace{\mathcal{E} \cdot \overline{\mathbf{J}}}_{\text {cascade }}=\epsilon_{\mathrm{sgs}}^{\mathrm{en}} .
\end{aligned}
$$

In this form the cross-scale cross-type transport is performed by $\nabla \cdot\left(\tau^{\mathrm{b}} \cdot \tilde{\mathbf{u}}\right)$, while the magnetic energy cascade is contained within $\mathcal{E} \cdot \overline{\mathbf{J}}$.

This dichotomy of forms is a signature of SGS filtering. Just as the SGS energy contains all the unresolved information pertaining to small scale and cross-scale interactions, the SGS energy flux cannot be uniquely decomposed into cascade and transport terms. This is also valid for the SGS transfer between the kinetic and magnetic budget. All we can assure is that the SGS stress transports energy between the resolved kinetic budget and the total SGS budget. Analogously, the EMF transports energy between the resolved magnetic budget and the total SGS one. This arbitrariness of decomposition makes the a priori testing of closures a delicate business. For instance, consider the local equilibrium hypothesis, which equates the local rates of SGS energy production and dissipation. Depending on the form of the term $\Sigma_{2}$ one obtains different answers for the SGS energy closure. The issue is compounded, if the hypothesis is invoked separately for the kinetic and magnetic energies. This delicacy motivates the wide selection of diagnostic parameters for the functional tests performed in chapter 4 .

\subsubsection{Term comparison}

In a manner similar to the primary equations let us analyse the relative importance of the various terms which enter the energy equations in light of the assumptions made in chapter 2. We start by examining the total SGS energy flux, shown in fig. 3.17. To gauge the global dynamics we take the time and space average of $\mathcal{F}^{\mathrm{en}}$ for each simulation over the statistically stationary period. We report without showing that the variations with time are small in comparison with the average value - the standard deviation is of the order of $10 \%$ of the mean. Figure 3.17 a shows that globally there is a forward cascade of energy from the resolved scales (since globally the transport terms average out), which becomes stronger with increasing Mach numbers. The presence of global forward cascade is not surprising since we are dealing with forced turbulence. Energy is constantly being supplied at the large scales and after all the transients have died out it can only decay down to smaller scales. Clearly, the rate of decay is guided by the driving amplitude. We show the trend with the RMS sonic Mach number. Locally, however, there is significant amount of backscatter, or inverse cascade. Figure 3.17b shows that the local distribution of $\mathcal{F}^{\mathrm{en}}$ is bimodal. The positive values correspond to SGS energy increase, i.e. direct cascade. The negative value correspond to decrease in the SGS 
energy, i.e. inverse cascade. It is evident that the local inverse cascade is about half as strong as the direct cascade. There seems to be a relationship with a quantity to which we shall refer with 'total RMS Mach number' $=\left\langle\sqrt{\left(\mathrm{M}_{\mathrm{s}}^{\mathrm{rms}}\right)^{2}+\left(\mathrm{M}_{\mathrm{a}}^{\mathrm{rms}}\right)^{2}}\right\rangle$, where again the RMS is taken with respect to space. When this quantity is small the distributions are rather symmetric and the global cascade is the result of cancellations across their bulk. As the total RMS Mach number increases the asymmetry between the tails of the forward and inverse cascade grows. It is the tail of the forward cascade that determines the global cascade rate in this case.

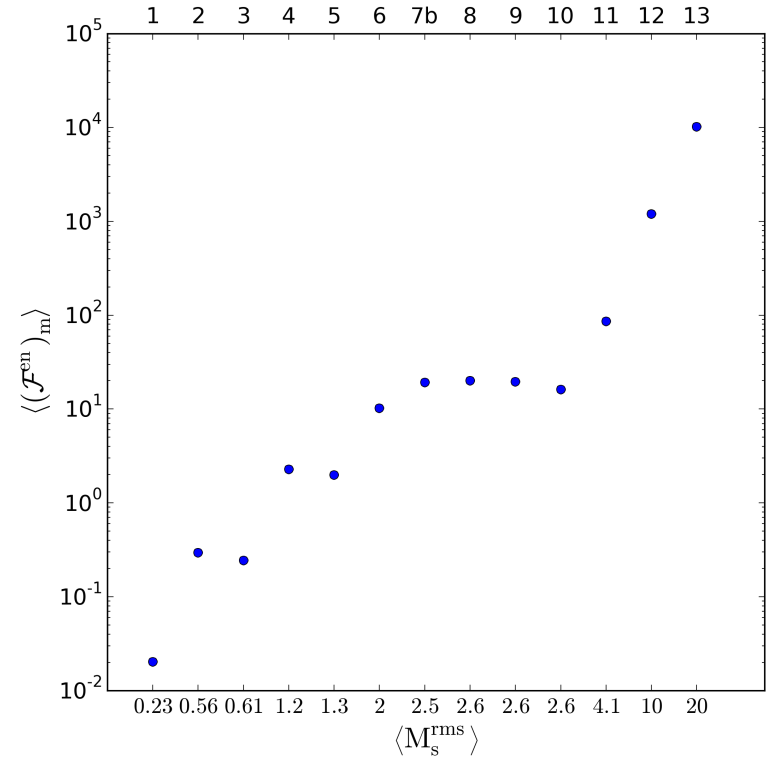

(a)

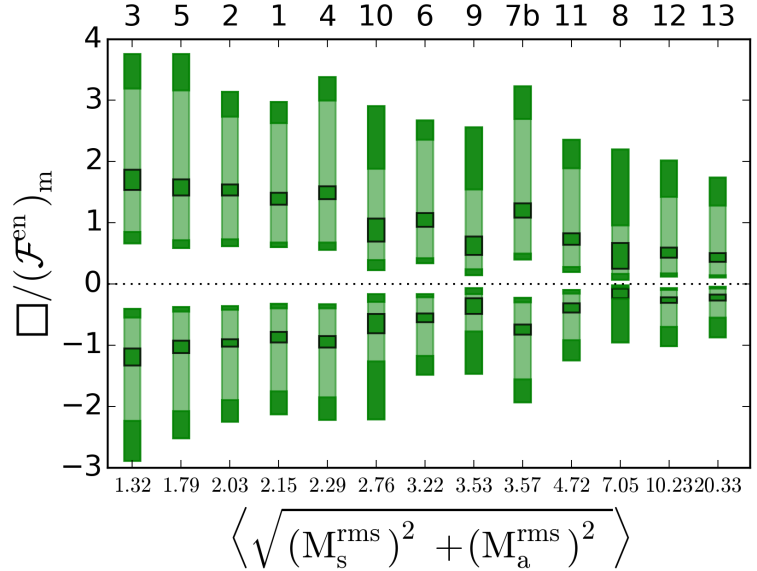

(b)

Figure 3.17: a)Total energy flux $\left\langle\left(\mathcal{F}^{\mathrm{en}}\right)_{\mathrm{m}}\right\rangle$ averaged over space and the statistically stationary time period $t \in[2 T, 5 T]$. The simulations are ordered by the RMS sonic Mach number. b) Local distribution of the total SGS energy flux normalised to the global average. The simulations are ordered by the 'total RMS Mach number'.

Let us consider next the components of $\mathcal{F}^{\mathrm{en}}$. Recall (section 2.3) that the Smagorinsky energy closures are derived from a local equilibrium hypothesis for the total SGS energy. The hypothesis implies that the left-hand side of eq. (3.14) is negligible. We test this by comparing the contributions from $\mathcal{F}^{\text {en,ther }}, \mathcal{T}^{\text {en }}$ and $\Sigma^{\text {en }}$. To gauge their global effect and variation we normalise them to the average total flux $\left\langle\mathcal{F}^{\mathrm{en}}\right\rangle$. Figure 3.18a confirms that as the total RMS Mach number grows the contribution from the thermal pressure become s negligible. In other words in supersonic and/or super-Alfvénic turbulence the overall effect, both diffusive and dissipative, of the thermal (dilatational) exchange between SGS and internal energy is negligible. As the RMS Mach numbers decrease the impact of the thermal pressure increases quite symmetrically in the direct and inverse transfer branches. It becomes stronger in the negative branch than the corresponding negative branch of $\Sigma^{\mathrm{en}}$. In the direct transfer branch however, the cascade term remains more prominent mainly by virtue of its flatter distribution. Thus, as intuition suggests, 
from the global point of view it is justifiable to neglect the thermal effect on the crossscale energy exchange for high Mach numbers but not for low ones.

The transport term $\mathcal{T}^{\text {en }}$ is also symmetric around 0 . The periodic boundary conditions trivially demand that the overall weight of the positive and negative branches cancel out. It is noteworthy, however, that the shape of the bulk of the distributions is also symmetric (compare the locations of the medians within the interquartile). This is a signature of the homogeneity and isotropy of the SGS energy transport - overall it does not create spatial concentrations or deficiencies of resolved or SGS energy. In the end, it is the cascade term $\Sigma^{\mathrm{en}}$ which determines the shape of the two branches of the $\mathcal{F}^{\mathrm{en}}$ distribution. Note the correspondence between the forward/backward cascade asymmetry in the distributions of $\Sigma^{\mathrm{en}}$ in fig. 3.18a and $\mathcal{F}^{\mathrm{en}}$ in fig. 3.17b.

The contributions of the three terms with respect to the local total SGS energy flux are shown in fig. 3.18b. The figure demonstrates that the dependence of $\mathcal{F}^{\text {en,ther }}$ on the plasma parameters is an intrinsic phenomenon, since it is retained here as well. On the other hand the behaviour of the cascade and transport terms is quite different. The width and median of their distributions seems to be quite insensitive to the variation of the plasma parameters. The distribution of $\mathcal{T}^{\mathrm{en}}$ shows that the diffusion of SGS energy away from a location is likely to take place in quite large packets (more than 3 times the local flux), while influx happens in smaller packets of typically the size of the local flux but seldom bigger than twice that size. Note that the simulations are ordered by the 'total average Mach number' $=\left\langle\left(M_{s}\right)_{m}+\left(M_{a}\right)_{m}\right\rangle$. As this quantity increases the diffusion of large energy packets become less likely - the distributions become a little more concentrated. It would be interesting to extend the range of simulations to higher Mach numbers. The current results cannot distinguish between reaching an asymptotic state, perhaps at equipartition, or complete reversal. Only in the latter case the SGS energy flux (i.e. the turbulent cascade) may be actually supported by the cascade terms. If the total Mach number is indeed the physical parameter driving this trend, the most computationally favourable conditions for this extension would be with magnetic Prandtl number of unity.

The cascade terms show even less dependence on plasma parameters than the transport ones. This might be a signature of a 'universal' mechanism at play, however based on this data we can only claim it is a signature of developed homogeneous and isotropic isothermal turbulence.

To complete the comparison between transport and cascade terms we consider directly the ratio $\Sigma^{\mathrm{en}} / \mathcal{T}^{\mathrm{en}}$. Figure $3.18 \mathrm{c}$ shows that locally the cascade is typically half as strong as the transport term. Moreover, they are equally likely to have the same effect as to counteract each other (the distributions are symmetric with respect to 0 ). While certainly there are regions in space where the cascade term dominates, they occupy typically about $25 \%$ of the simulation volume. The figure also confirms that as the sonic and Alfvénic Mach numbers increase, the regions with local cascade dominance become somewhat more likely. It further suggests that while the flattening of the distribution may continue the median does not shift significantly. In other words most frequently in the simulation domain the cascade term is sub-dominant.

To summarize, within the investigated parameter range the turbulent transfer of energy across the filter scale is actually a Lagrangian property brought about by transport 
terms $^{5}$. We can picture the action of these terms as re-arranging flow elements like pieces in a jigsaw puzzle, creating and destroying large scale patterns (e.g. flows) but not really deforming the individual pieces. The cascade terms on the other hand are Eulerian in nature. They are responsible for the deformation of individual elements but not their transport. They create and destroy large-scale patterns by bringing the local flow in and out of coherence with its environment. In this sense, for the parameter regime under investigation, MHD turbulence is largely Lagrangian in nature. This suggests that the modelling of the SGS terms needs to be focused on large scale structure and geometry rather than local deformation. In that sense we expect that the functional models might have difficulties in this parameter range, since they are centred around the local cascade paradigm.

Next, we consider the possibility that at least parts of the local equilibrium hypothesis holds. We can separate the SGS energy flux by the order of the SGS terms. If the third order term $\mathcal{F}_{3}^{\text {en }}$ can be eliminated from consideration or absorbed into the model of the second order term, the closure problem would be greatly alleviated. Moreover, as we saw in section 2.3.1 closing the SGS energy can be done by solving its evolution equation explicitly and obtaining accurate local values. This of course would be cheaper and simpler, if the closures of $\mathcal{F}_{3}^{\text {en }}$ are derived from the ones of $\mathcal{F}_{2}^{\text {en }}$, or better yet, if these terms are negligible. From fig. 3.19 we see however, that locally both terms are of the same characteristic size and their distributions have similar widths. Interestingly the distributions are much flatter in the simulations with lower $\left\langle\beta_{\mathrm{p}}\right\rangle$. The third order term tends to have larger characteristic value and broader distribution on the inverse cascade branch than the second order terms. The positive energy flux on the other hand is on average equally distributed between the second and third order terms. Thus omitting even just $\mathcal{F}_{3}^{\text {en }}$ from an equilibrium hypothesis is not justifiable. With that we conclude that the local equilibrium hypothesis fails entirely for the total SGS energy equation.

We can still separate the evolution equations for the kinetic and magnetic SGS energies and examine them individually. In fig. 3.20 we show the local importance of the second order cascade term $\Sigma_{2}^{\square}$ with respect to the remainder $\mathcal{F}^{\square}-\Sigma_{2}^{\square}=\mathcal{T}_{2}^{\square}-\mathcal{F}_{3}^{\square}$. We notice behaviour analogous to the total SGS energy. $\Sigma_{2}$ is characteristically half as strong as the remainder both when it acts with and against $\mathcal{F}-\Sigma_{2}$. We conclude that the local equilibrium hypothesis also fails separately for the kinetic and magnetic SGS energies.

\footnotetext{
${ }^{5}$ We can not exclude the possibility that the key parameter in this transition is different from the total Mach number and therefore a different behaviour might be accessible for these Mach numbers as well.
} 


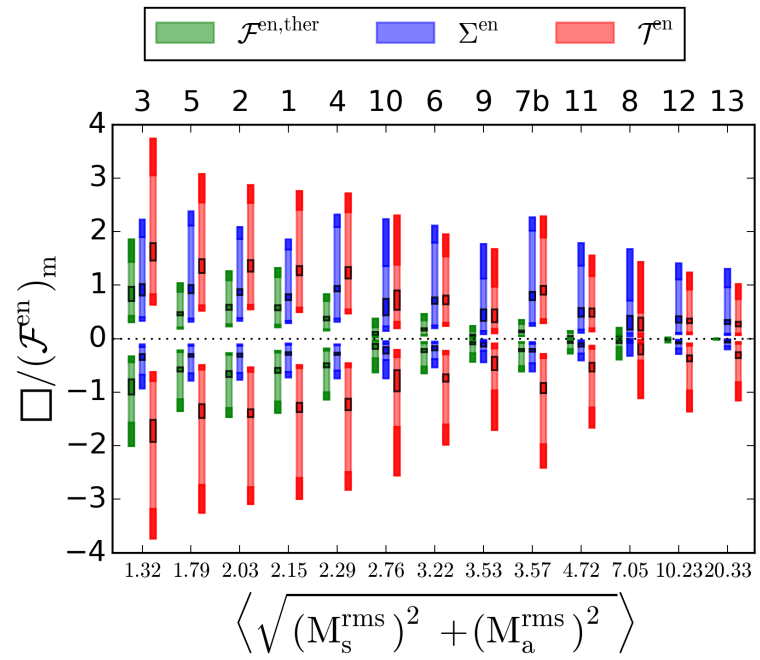

(a)

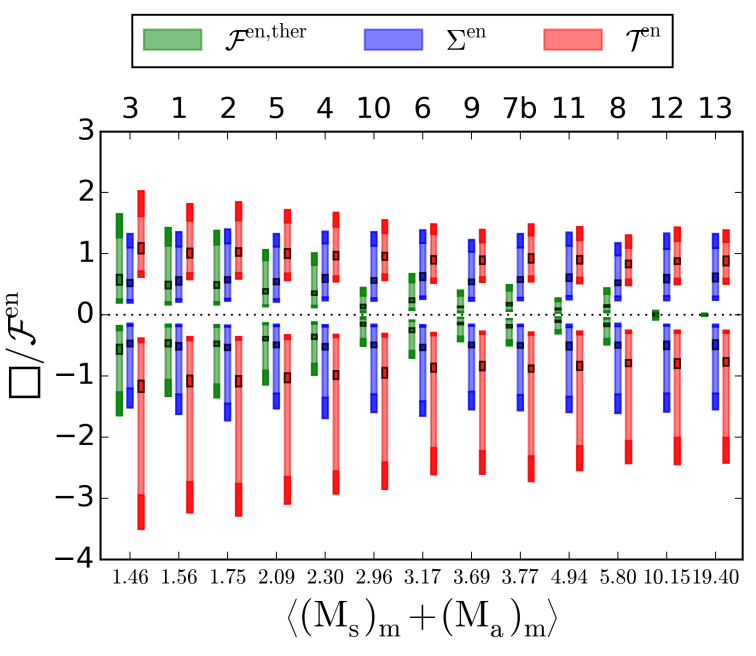

(b)

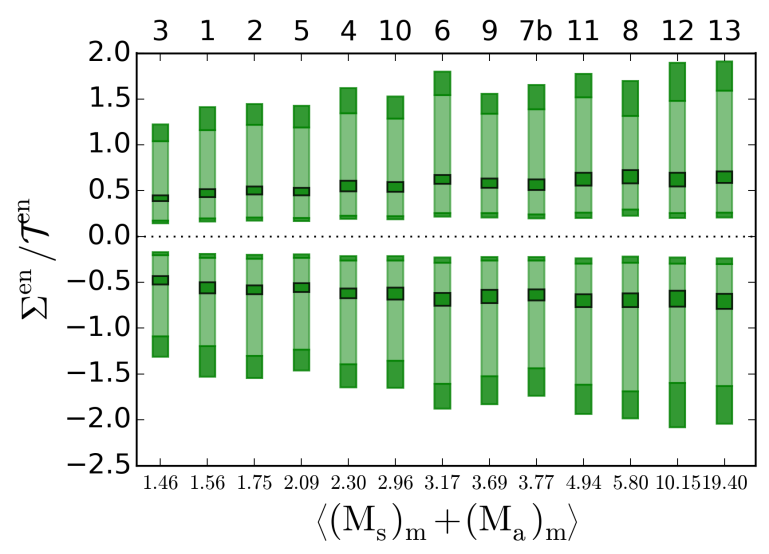

(c)

Figure 3.18: Comparison of the total transport, source and thermal terms from the total SGS energy equation. a) Global normalisation to the average SGS energy flux. b) Local normalisation to the SGS energy flux. c) Local ratio of cascade to transport term.

\subsection{Cross-helicity equation}

We proceed with the analysis of the cross-helicity equation in an analogous fashion. The resolved and SGS equations take the form

$$
\begin{array}{r}
\frac{\partial}{\partial t} H_{\mathrm{res}}+\nabla \cdot\left(\tilde{\mathbf{u}} H_{\mathrm{res}}-\frac{\overline{\mathbf{B}}}{\bar{\rho}} E_{\mathrm{res}}^{\mathrm{u}}\right)+\nabla \cdot\left(\tilde{\mathbf{u}} \times \mathcal{E}+\frac{\overline{\mathbf{B}}}{\bar{\rho}} \cdot \tau\right)= \\
=-\frac{\overline{\mathbf{B}}}{\bar{\rho}} \cdot \nabla \bar{P}+\mathcal{E} \cdot \tilde{\boldsymbol{\Omega}}+(\tau \cdot \nabla) \frac{\overline{\mathbf{B}}}{\bar{\rho}},
\end{array}
$$




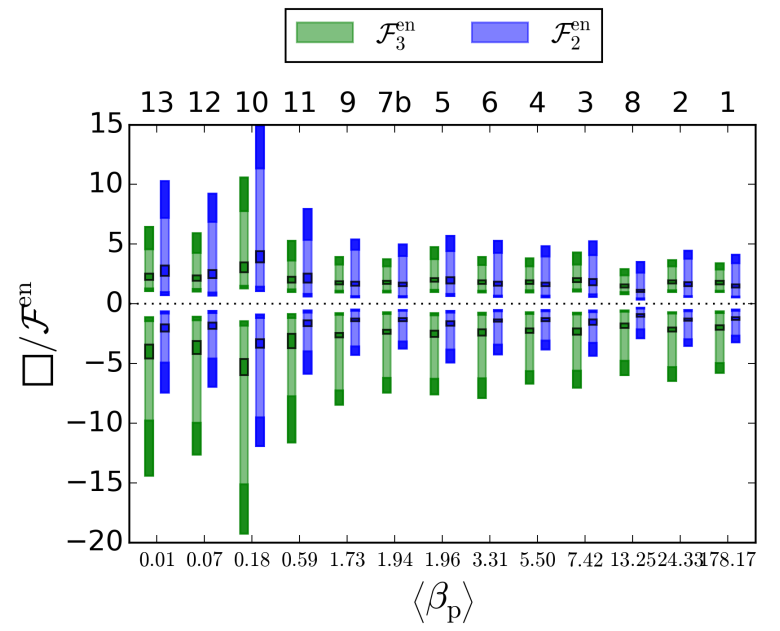

Figure 3.19: Comparison of the total transport 2nd and 3rd order terms normalised locally to the total energy flux.

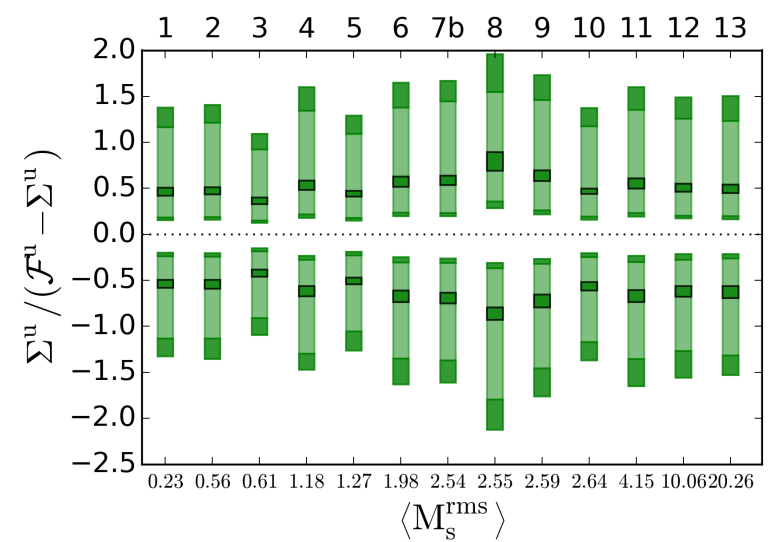

(a)

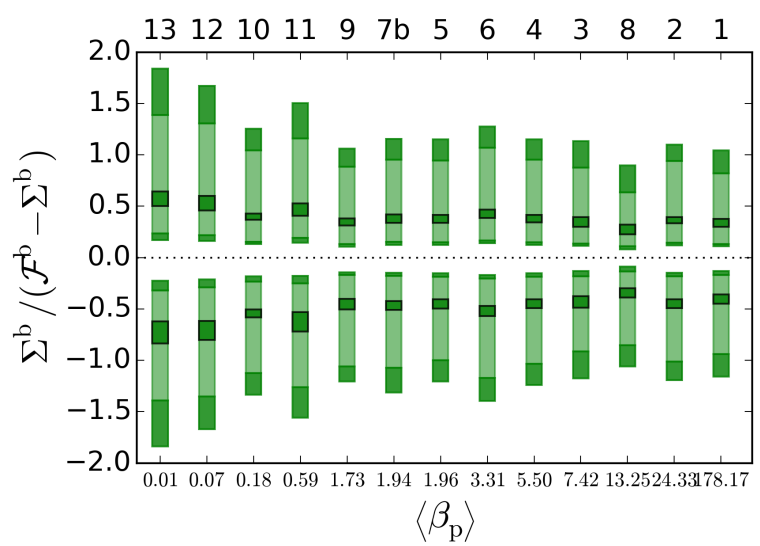

(b)

Figure 3.20: Investigation of the local equilibrium approximation for the kinetic and magnetic SGS energies.

$$
\begin{aligned}
\frac{\partial}{\partial t} H_{\mathrm{sgs}} & +\nabla \cdot\left(\overline{\mathbf{u} H}-\tilde{\mathbf{u}} H_{\mathrm{res}}-\left(\overline{\frac{\mathbf{B}}{\rho} E^{\mathrm{u}}}-\frac{\overline{\mathbf{B}}}{\bar{\rho}} E_{\mathrm{res}}^{\mathrm{u}}\right)\right)-\nabla \cdot\left(\tilde{\mathbf{u}} \times \mathcal{E}+\frac{\overline{\mathbf{B}}}{\bar{\rho}} \cdot \tau\right)= \\
& =-\left(\overline{\frac{\mathbf{B}}{\rho} \cdot \nabla P}-\frac{\overline{\mathbf{B}}}{\bar{\rho}} \cdot \nabla \bar{P}\right)-\mathcal{E} \cdot \tilde{\boldsymbol{\Omega}}-(\tau \cdot \nabla) \frac{\overline{\mathbf{B}}}{\bar{\rho}} .
\end{aligned}
$$


We label the terms as follows:

$$
\begin{aligned}
\Sigma^{\mathrm{ch}}=\Sigma_{2}^{\mathrm{ch}} & =-\mathcal{E} \cdot \tilde{\mathbf{\Omega}}-(\tau \cdot \nabla) \frac{\overline{\mathbf{B}}}{\bar{\rho}} \\
\mathcal{T}_{2}^{\mathrm{ch}} & =\nabla \cdot\left(\tilde{\mathbf{u}} \times \mathcal{E}+\frac{\overline{\mathbf{B}}}{\bar{\rho}} \cdot \tau\right) \\
\mathcal{F}^{\mathrm{ch}, \text { ther }} & =\frac{\overline{\mathbf{B}} \cdot \nabla P}{\rho}-\frac{\overline{\mathbf{B}}}{\bar{\rho}} \cdot \nabla \bar{P} \\
\mathcal{T}_{3}^{\mathrm{ch}} & =\nabla \cdot\left(\overline{\mathbf{u} H}-\tilde{\mathbf{u}} H_{\mathrm{res}}\right)-\nabla \cdot\left(\frac{\overline{\mathbf{B}}}{\rho} E^{\mathrm{u}}-\frac{\overline{\mathbf{B}}}{\bar{\rho}} E_{\mathrm{res}}^{\mathrm{u}}\right) \\
\mathcal{F}_{3}^{\mathrm{ch}} & =\mathcal{F}^{\mathrm{ch}, \text { ther }}+\mathcal{T}_{3}^{\mathrm{ch}}
\end{aligned}
$$

Again $\Sigma^{\mathrm{ch}}$ denotes the local cascade from resolved to subgrig scale, $\mathcal{T}^{\mathrm{ch}}=\mathcal{T}_{2}^{\mathrm{ch}}-\mathcal{T}_{3}^{\mathrm{ch}}$ denotes diffusion and $\mathcal{F}^{\mathrm{ch}}=\Sigma^{\mathrm{ch}}+\mathcal{T}^{\mathrm{ch}}$ is total SGS cross-helicity flux ${ }^{6}$.

We go through the same steps as we did for the SGS energy in order to establish the importance of the transport versus cascade terms and the third versus second order terms. Figure 3.21a shows the dependence of the average cross-helicity flux on plasma parameters. We see that hypersonic driving produces larger cross-helicity flux, specifically from the SGS to the resolved scales. The local distribution of the flux, fig. $3.21 \mathrm{~b}$, reveals very strong variations in time, comparable to the variations in space. This implies that these distributions are contaminated by large transients. We follow their origin to the effect of large density gradients. Note that, unlike in the energy equations, here the compressibility effects enter through the inverse of the resolved density $\bar{\rho}^{-1}$, allowing for local singularities. Thus strong shocks can strongly disturb the quiescent dynamics. They force us to forego the analysis of the absolute distributions of the flux constituents, as they also are contaminated by the same noise. Thus we will only show the local normalisation to $\mathcal{F}^{\text {ch }}$.

Figure $3.22 \mathrm{~b}$ demonstrates the the local statistics is not affected by the transients (no big time variations are evident). This implies that the transient behaviour follows the same local SGS dynamics as the stationary/quiescent phase.

Interestingly, the distribution of the transport term is no longer symmetric with respect to 0 . This means that the cross-helicity grows to large scales more frequently through large local jumps, while the decay happens at smaller steps. The cascade mechanism is approximately symmetric and much weaker. Figure $3.22 \mathrm{~b}$ shows that locally the cascade is sub-dominant for almost all simulations in most of the simulation domain with characteristic ratio of approximately $1 / 3$.

Finally, we consider the separation between second and third order terms in fig. 3.23. Note that we have included the pressure term $\mathcal{F}^{\text {ch,ther }}$ within the third order terms ${ }^{7}$. The strong transients are revealed once more. This suggests that they are driven by

\footnotetext{
${ }^{6}$ Here, there is the same freedom in the separation between the diffusion and cascade terms as for the SGS energies.

${ }^{7}$ Depending on the equation of state, it is at least of third order. Therefore it might be more accurate to think of $\mathcal{F}_{3}^{\text {ch }}$ as a 'higher order' term.
} 


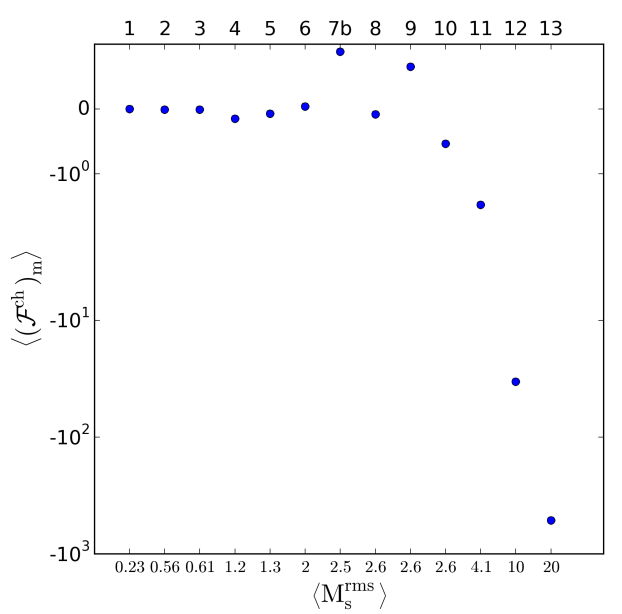

(a)

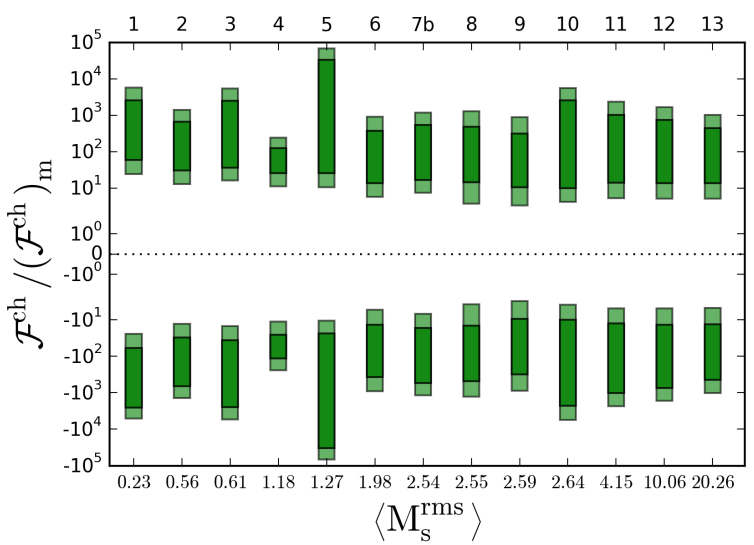

(b)

Figure 3.21: a) For each simulation the SGS cross-helicity flux is averaged over the simulation domain, $\left\langle\mathcal{F}^{\mathrm{ch}}\right\rangle$. We take the mean of the result over the saturated time period $t \in[2 T, 5 T]$. The simulations are ordered by the driving sonic Mach number. b) Local distribution of the SGS cross-helicity flux normalised to the global average. The darker bar represents the time variation of the median, the lighter bar - the maximum extent of the interquartile over the time period, i.e. the minimum of the first quartile and the maximum of the last quartile.

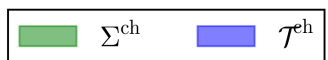

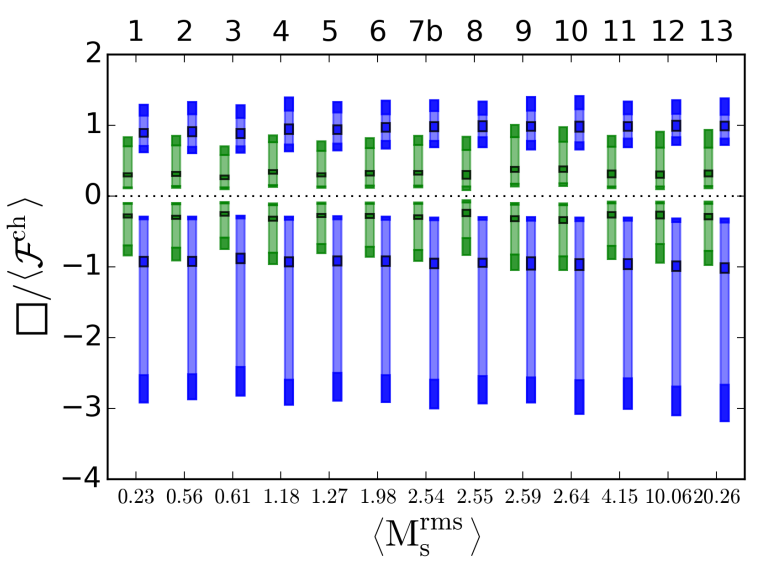

(a)

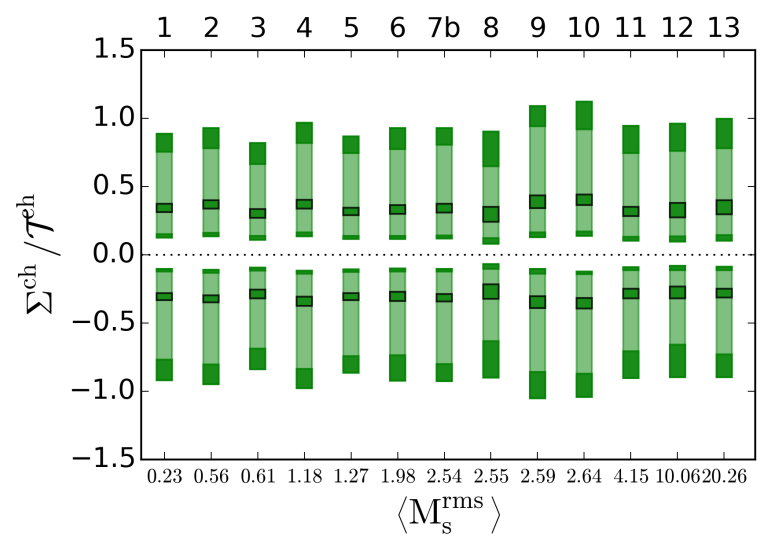

(b)

Figure 3.22: Comparison of the total transport and source terms in all simulations: a) the distributions relative to the local SGS cross-helicity flux, b) the direct ratio of $\Sigma^{\mathrm{ch}} / \mathcal{F}^{\mathrm{ch}}$.

the thermal pressure, which in isothermal conditions translates to logarithmic density gradients, i.e. strong shocks. Therefore the $\mathcal{F}_{2}^{\text {ch }}$, specifically $\mathcal{T}_{2}^{\text {ch }}$, is more sensitive to the local transient events, while $\Sigma_{2}^{\text {ch }}$ follows the quiescent dynamics. For this reason we shall use both as diagnostic variables for functional tests of the SGS closures. 


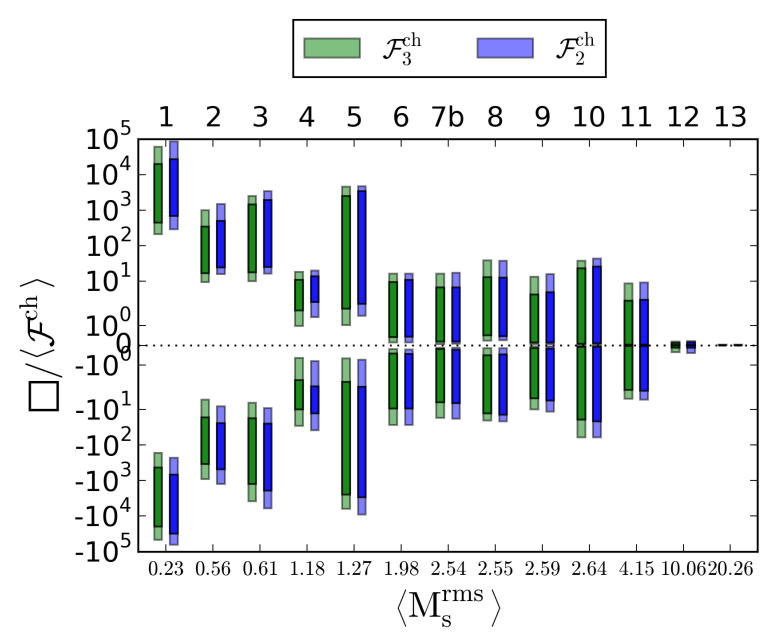

Figure 3.23: Comparison of $\mathcal{F}_{2}^{\text {ch }}$ and $\mathcal{F}_{3}^{\text {ch }}$ normalised locally to the total flux term $\mathcal{T}^{\text {tot }}$. The darker bar represents the time variation of the median, the lighter bar the maximum extent of the interquartile over the time period, i.e. the minimum of the first quartile and the maximum of the last quartile. 


\section{Model Validation}

In the last chapter we noted some of the properties of the SGS terms in the LES evolution equations. Considering the wide variety of physical effects they represent, an SGS model has to conform with well more than just one of them in order to be considered successful and robust. In this chapter we compare the behaviour of the various models described in chapter 2 with regards to these properties.

The comparisons are performed on the sub-parts of the SGS terms, i.e. for the components of the SGS stress tensor we consider separately the isotropic parts $E_{\mathrm{sgs}}^{\mathrm{u}}, E_{\mathrm{sgs}}^{\mathrm{b}}$ and $P_{\text {sgs }}$ and the deviatoric parts $\tau^{\mathrm{u} *}, \tau^{\mathrm{b} *}$ and $\tau^{*}$. Comparing models of individual terms circumvents the possibility of error cancellation between them. Thus the performance of individual closures can be clearly established. It should be noted however that this also means that the corresponding model for the combined term, e.g. $\tau$, might perform better than the constituent closures. Considering the total isotropic and deviatoric contributions gauges some of the extent of this improvement. However, it should be noted that if such improvement takes place, the reason for it would lie outside the model assumptions and motivation. Hence it may not be reliable or robust in different regions of the parameter space.

In order to obtain robust results comparing frame independent quantities, i.e (pseudo) scalars, is preferable. Due to the predominantly functional nature of existing models we focus on functional tests. Since the SGS energies are already scalar fields, we compute for them direct correlations between model and data. However, for non-scalar fields we cannot employ such a method. Instead, we measure the models' ability to capture the SGS energy and cross-helicity flux across the filter scale. In other words, we measure the contributions of the SGS terms to the LES energy and cross-helicity equations ${ }^{1}$. Because of the presence of derivatives in these contributions, they provide a physical non-local measure of the goodness-of-fit of the models. In the last chapter, sections 3.4 and 3.5, we showed the arbitrariness of the decomposition of the flux into cascade and transport components. It motivates the investigation of 4 distinct diagnostic flux fields. The total transport energy and cross-helicity terms $\mathcal{F}_{2}^{\text {en }}=\tilde{\mathbf{u}} \cdot(\nabla \cdot \tau)+\overline{\mathbf{B}} \cdot \nabla \times \mathcal{E}$ and $\mathcal{F}_{2}^{\text {ch }}=(\overline{\mathbf{B}} / \bar{\rho}) \cdot(\nabla \cdot \tau)+\tilde{\mathbf{u}} \cdot \nabla \times \mathcal{E}$ capture the total contribution of the second-rank SGS terms to the evolution equations and thus present a robust picture of the overall goodness-of-fit of the models. They can be interpreted as representing the local transfer between scales from an Eulerian point of view. Conversely, the corresponding cascade parts $\Sigma_{2}^{\mathrm{en}}=\tau \cdot \tilde{\mathcal{S}}+\mathcal{E} \cdot \overline{\mathbf{J}}$ and $\Sigma_{2}^{\mathrm{ch}}=\tau \cdot \nabla(\overline{\mathbf{B}} / \bar{\rho})+\mathcal{E} \cdot \tilde{\Omega}$, may be interpreted as the part of the local transfer as seen from a Lagrangian point of view. The latter remains however not uniquely (or rigorously) defined. Its significance is related to the Smagorinsky

${ }^{1}$ As the second rank SGS terms appear with opposite signs in the resolved and SGS equations, it is immaterial which equation is chosen. 
approximation of local balance on which the EV/ER/ED models are largely based. In the available data the cascade part does not play a dominant role, however we cannot exclude such a possibility for different flow configurations, e.g. decaying or non-homogeneous, anisotropic turbulence.

Additionally, since the cascade terms involve the pure SGS terms while the total flux terms involve their derivatives, the comparison between the two will reveal the extent of the commutation error between the various modelling strategies and differentiation. Recall that although the direct filtering operation commutes with differentiation, Favre filtering does not. There is a commutation error which for a general derivative of a velocity field component is given by

$$
\tilde{u}_{i, j}-\widetilde{u_{i, j}}=\frac{\overline{u_{i} \rho_{, j}} \bar{\rho}-\overline{u_{i} \rho} \bar{\rho}_{, j}}{\bar{\rho}^{2}}
$$

We shall refer to this term as the commutator between Favre filtering and differentiation. How well this term is captured by the various models can be seen in the comparison between cascade and full flux-term fits.

With all this in mind we proceed by examining the correlations between data and model of the contributions of the different SGS terms to all four diagnostic fields. Since the cross-scale transfer determines fundamentally the strength of the SGS effect on the resolved scales we use fits to the diagnostic fields in order to determine values of the free coefficients in the various models. The best fit is sought in the least-square sense via the Levenberg-Marquardt algorithm (see e.g. [57]) independently for each diagnostic field and simulation snapshot. The coefficient values will not have a strong impact on the following analysis, especially for single coefficient models, as we focus on the performance of the functional form of the models. Therefore, as this work is aimed at an a posteriori application of the models, we limit ourselves to observations of the coefficients' overall variation within the dataset, i.e with respect to time, simulation and diagnostic variable. This we take as a measure of the robustness and completeness of the functional form of the respective model.

We follow up the functional comparison with a structural examination which sheds some light on the reasons for the models' performance. At the vector level, i.e. for the SGS forces, we have noted that there is little variation of the absolute orientation with respect to time and simulations. Since this makes the geometrical distribution of the SGS forces a robust feature of SGS turbulence, we measure the corresponding alignment angle between data and model. The accompanying scalar diagnostic is the size of the error vector between the data and model. Together they completely determine the geometry of the modelling error. The corresponding measure for tensor quantities are the quadratic invariants. We therefore compute the distribution of the model among the 4 topological regions described in section 3.3.1. and compare it to the data. As we saw this is also a robust feature of the deviatoric SGS stress.

This chapter is organised as follows. We begin by examining the closures of the SGS energies. We then proceed to investigate the closures of the SGS stress and finally finish with the EMF. 


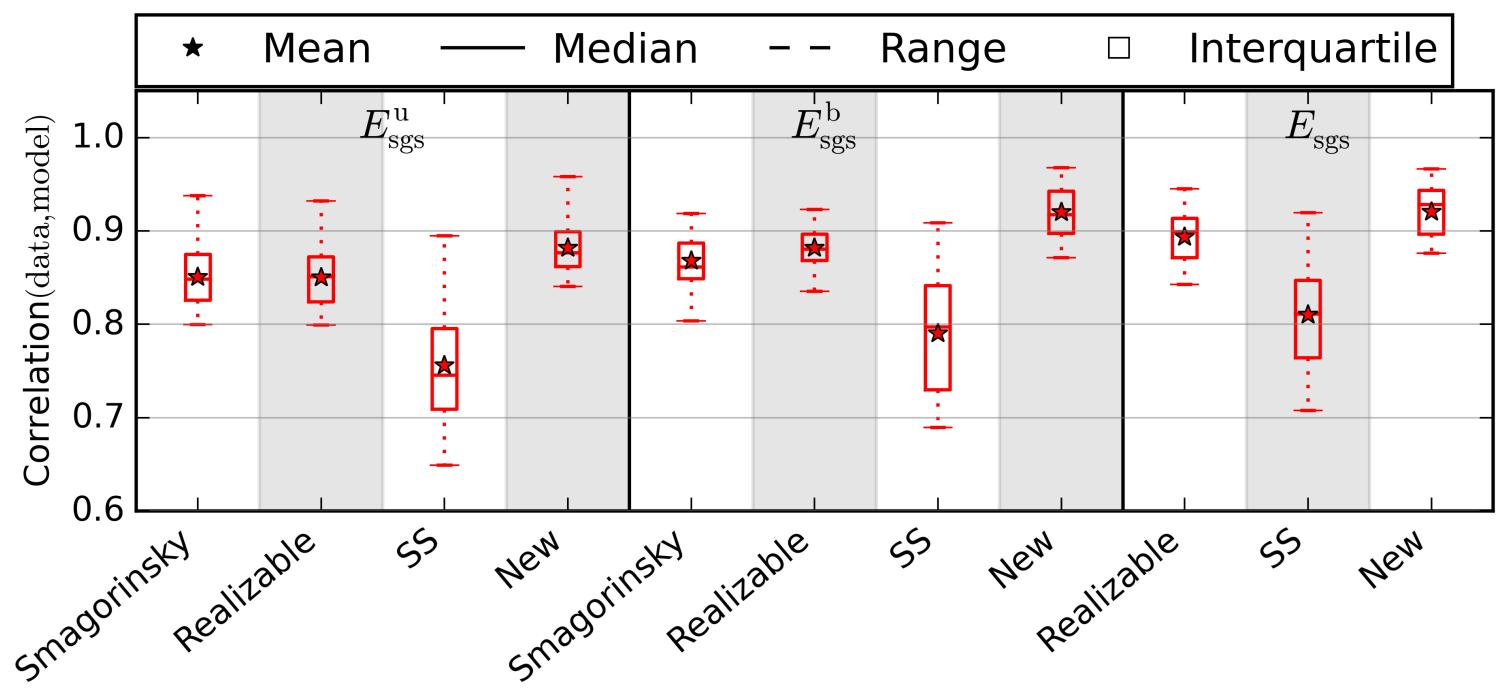

Figure 4.1: Correlation between data and model of the SGS kinetic, magnetic and total energies. The models are labelled as in table 2.4. The total variation over all simulations within the statistically stationary period is given by the whiskers (dotted lines), the box represents the interquartile, the horizontal bar inside it - the median and the star designates the mean.

\subsection{Sub-grid scale energy models}

The models of the kinetic and magnetic SGS energies we consider are listed in table 2.4. We additionally construct the respective models for the total SGS energy as a linear combination with two freely varying coefficients. To summarize the correlations between model and data for all snapshots, we use a modified box-and-whisker plot. The box represents the interquartile, the whiskers cover the entire range of variation. To give a sense of the shape of the distribution we further mark the mean and the median correlation for each model. The results are presented in fig. 4.1.

Overall all models give a reasonable approximation to the SGS energies. The scalesimilarity (SS) models perform most poorly and have the largest variation across the dataset. Since they are based on assumptions completely unrelated to the SGS energy, it is actually remarkable, that the average correlation is above 0.7 for all energy species. The realizable and Smagorinsky models for the kinetic and magnetic SGS energies perform almost identically. So much so that for the total energy fit we only use one of the two options, namely the realizable one. The new model outperforms them consistently by a small margin. Thus, we conclude that any of the three can be used as a local and instantaneous approximation to the SGS energies.

To extend this statement in time and space (in order to allow the use of the closures under differentiation for instance), we consider in fig. 4.2 the contribution of the SGS energies to the four diagnostic flux terms $\Sigma_{2}^{\mathrm{en}}, \Sigma_{2}^{\mathrm{ch}}, \mathcal{F}_{2}^{\mathrm{en}}$ and $\mathcal{F}_{2}^{\text {ch }}$. Since they enter through the trace of the SGS stress, we replace the model of the total SGS energy with a model of the total SGS pressure $P_{\text {sgs }}$ (i.e. $\operatorname{Tr}(\tau)$ ). This corresponds simply to a rescaling of the kinetic energy contribution by a factor of 2 , which does not affect 


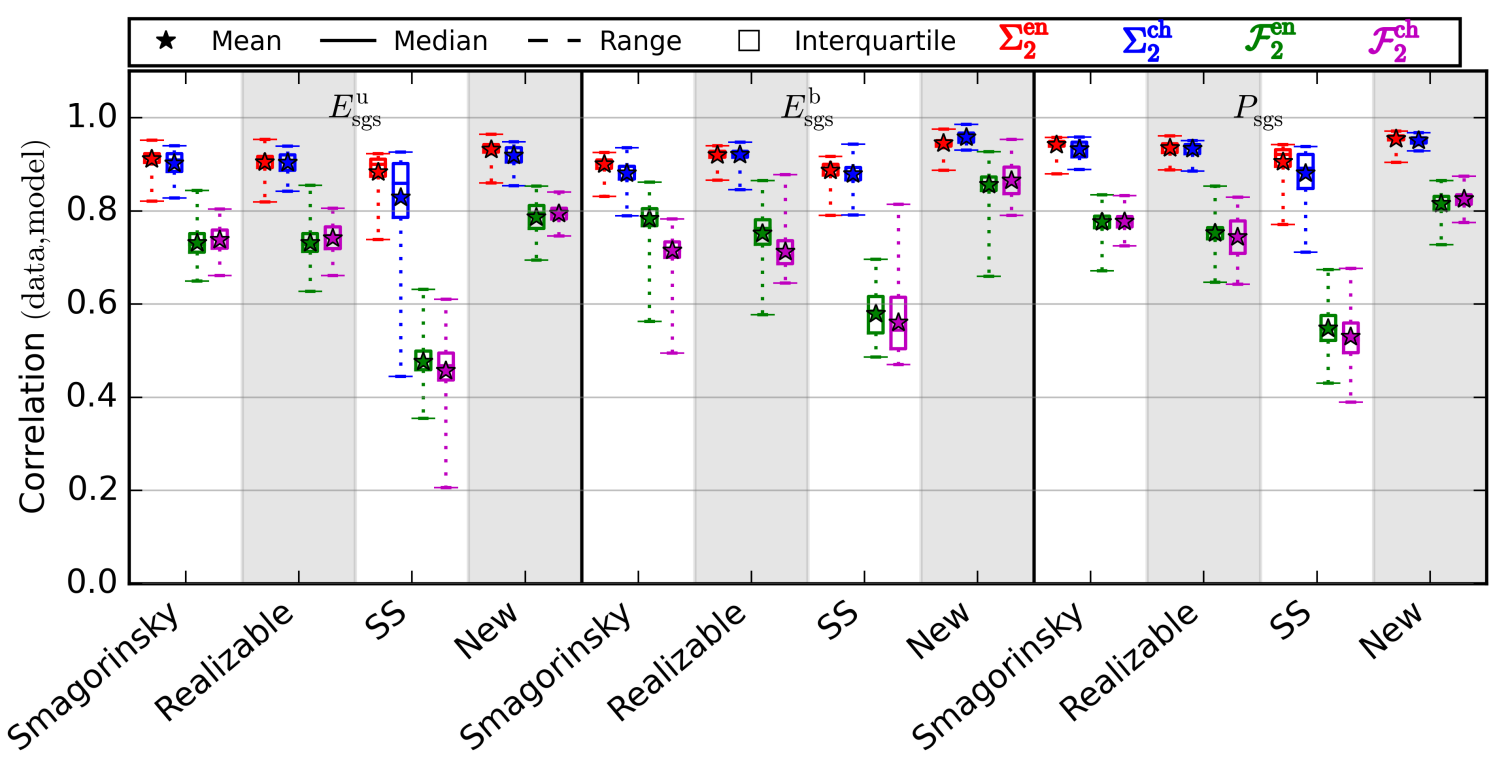

Figure 4.2: Correlation between data and model of the contributions of the isotropic terms to the four diagnostic fields $\Sigma_{2}^{\text {en }}$ (red), $\Sigma_{2}^{\text {ch }}$ (blue), $\mathcal{F}_{2}^{\text {en }}$ (green) and $\mathcal{F}_{2}^{\text {ch }}$ (magenta). The models are grouped by types: kinetic energy, magnetic energy and pressure and labelled as in table 2.4 .

the correlations' behaviour. Thus the performance of the SGS pressure models can be used to evaluate the corresponding total energy models. Since the SGS pressure carries the total isotropic contribution of the SGS stress, it measures the overall quality of the respective isotropic models. The individual kinetic and magnetic energy fits, on the other hand, demonstrate how the quality of the direct fit is propagated through the evolution equations ${ }^{2}$. We see that it is maintained for the cascade contributions, especially for the $\Sigma_{2}^{\mathrm{en}}$ fit. However, there is a trend which will be evident in the models of the rest of the SGS terms as well - the correlations with the total flux terms are significantly lower than the ones with the cascade counterpart. This is unsurprising for the Smagorinsky energies (and the very similar realizable ones), after all they are built to follow specifically the energy cascade term and carry no information about the SGS derivatives or the cross-helicity terms. However, it is not entirely expected in the structural models. It indicates that these closures don't capture well a very important process - diffusion, specifically its commutator with Favre filtering.

In the case of the new models, if we propagate the commutator between Favre filtering and differentiation through the Yeo-Bedford expansion, it yields contributions of order ${ }^{3}$ $O(a)$ in the models of the SGS forces (including $\nabla \times \mathcal{E}$ ). Since this is their leading order, these contributions have a strong impact on the total flux fits, but can not be detected in a pure cascade comparison. Recalling that the SGS terms enter all LES equations under a differential operator, this is an important extension which needs to

\footnotetext{
${ }^{2}$ This is because the flux terms carry information about the effect of the SGS energies on the SGS energies' evolution.

${ }^{3}$ Recall that $a=\Delta^{2} / 12$ is related to the second moment of the filter kernel.
} 
be included in a posteriori applications. From the a priori validation presented here we can anticipate the effect that such an extension will have by comparing the total flux and cascade correlations. In the case of the SGS kinetic energy gradient the extension is given by

$$
\widehat{\partial_{i} E_{\mathrm{sgs}}^{\mathrm{u}}}-\partial_{i} \widehat{E}_{\mathrm{sgs}}^{\mathrm{u}}=2 a \bar{\rho} \partial_{l}\left(\tilde{u}_{k} \tilde{u}_{k}\right) \partial_{i} \partial_{l}(y),
$$

Recall that $y=\ln \bar{\rho}$ designates the natural logarithm of the resolved density. Here and in the following we indicate the modelling operation with ?

Let us compare the performance of the individual models. We note that the scalesimilarity closures again have the lowest correlations and lead to the largest variation across the dataset. They perform especially poorly with respect to the cross-helicity. The fit to the SGS pressure is somewhat better than the ones to the individual energies. This can be attributed to the extra degree of freedom of the two-coefficient fit. If we consider the different simulations individually (not shown), the correlations to the cascade terms decrease slowly but consistently with increasing $\mathrm{M}_{\mathrm{s}}$ for the $E_{\mathrm{sgg}}^{\mathrm{u}}$ and $P_{\mathrm{sgs}}$ models, but remain constant for the $E_{\mathrm{sgs}}^{\mathrm{b}}$ models. With respect to the total flux terms this trend is enhanced for the supersonic runs. In the sub- and trans-sonic ones however there is a slight improvement of the correlation with increasing sonic Mach number. This implies that the scale-similarity model is best suited for trans-sonic simulations with moderate plasma beta $\beta_{\mathrm{p}}$ (see table 1.2).

The Smagorinsky, realizable and new models perform similarly to each other with respect to the cascade terms. The correlation does not seem to depend strongly on the plasma parameters since the variations are quite small and unordered with a few notable exceptions. The kinetic energy fit to $\mathcal{F}_{2}^{\text {ch }}$ of the Smagorinsky model improves consistently in the subsonic regime. The kinetic energy and SGS pressure fits to $\mathcal{F}_{2}^{\text {ch }}$ of the realizable model show the same behaviour. The kinetic energy correlations of the new model decrease slowly with increasing $\mathrm{M}_{\mathrm{s}}$. Its magnetic energy correlations however increase, especially for the supersonic runs. As a result, the total pressure correlations show much smaller variability. It should be noted that the bulk of the variation in $\mathcal{F}_{2}^{\text {en }}$ seems to stem from a few snapshots in the trans-sonic simulations. Overall, it is clear that the new model is better equipped to deal with the total flux terms.

It is interesting, albeit not immediately evident, that for the Smagorinsky, realizable and new models the performance of the SGS pressure is similar to the one of the kinetic SGS energy in the cascade terms but resembles more closely the performance of the magnetic SGS energy in the total flux terms. We have checked that this behaviour is not related to the value of the coefficients. With the exception of the scale-similarity models they all vary by a factor of order unity and the bulk of variation is again due to isolated snapshots in the trans-sonic runs.

We repeated this analysis for a test-filter scale of $k=8$ and $k=32$, which fall closer to the forcing and dissipation edges of the inertial range, with the same qualitative results (not shown). The overall range of variation is unchanged, at $k=32$ the bulk of the correlations tend to increase, which is unsurprising since there is very little remaining unresolved information. At $k=8$ the reverse is observed. 
Based on this analysis we discard the scale-similarity models and retain the rest as viable closures for the SGS energies and pressure. The latter can be used directly in LES and the former - as approximations within the models of the other SGS terms which require the SGS energy.

\subsection{Deviatoric SGS kinetic stress: $\tau^{\mathrm{u} *}$}

Let us now consider the performance of the models of $\tau^{\mathrm{u} *}$. We identify 4 families of models of the deviatoric kinetic SGS stress - TSDIA/EV (tables 2.2 and 2.3), scalesimilarity (SS), new (table 2.1) and mixed. The TSDIA/EV models are characterised by the form $\widehat{\tau}_{*}^{\mathrm{u}} \sim \nu_{\mathrm{u}} \tilde{\mathcal{S}}^{*}$. They are labelled by the characteristic variable which determines the eddy-viscosity $\nu_{\mathrm{u}}$, i.e. constant, cross-helicity or energy. We include here the two cross-helicity models from table 2.2 although they were introduced as models for the full traceless SGS stress $\tau^{*}$. They have the same tensorial structure as the TSDIA models, so we simply consider them as ad hoc expressions for the eddy-viscosity. The energy-based eddy-viscosity comes in three forms: using the true SGS kinetic energy $E_{\text {sgs }}^{\mathrm{u}}$, its realizable closure $\propto\left\|\tilde{\mathcal{S}}^{*}\right\|^{2}$ and Smagorinsky closure $\propto\|\tilde{\mathcal{S}}\|^{2}$. Similarly, the new model comes in its basic form as well as renormalized by the SGS kinetic energy or one of its closures. Finally, the mixed models consist simply of a linear combination of the new and the energy-based eddy-viscosity model, whereby both are renormalized either by the true or by the realizable SGS kinetic energy. Note that therefore the mixed models have two freely fitted coefficients. This additional degree of freedom incurs an extra cost, in an a posteriori implementation for instance. To compensate for that the mixed models should perform significantly better than the single coefficient models.

Figure 4.3 shows an overview of all the models' performance with respect to the four diagnostic fields. As for the SGS energies, the correlations to the full flux terms are consistently worse than the ones to the cascade terms. Thus the $\tau^{\mathrm{u} *}$ models also do not capture well the commutation of differentiation with Favre filtering. Considering the individual model families we observe the following.

The correlations for the TSDIA/EV models vary significantly between simulations. Unsurprisingly the diagnostic field reaching the highest correlations for these models is $\Sigma_{2}^{\mathrm{en}}$. However it also exhibits some of the poorest correlations. Crucially, the overall behaviour seems to be quite insensitive to the shape that the eddy-viscosity takes. The fits to the total energy and cross-helicity flux is the poorest for the majority of snapshots.

In comparison the scale-similarity model has approximately the same maximum correlation for $\Sigma_{2}^{\text {en }}$, however the minimum is much higher, making this a preferable solution to the TSDIA/EV models. While the scale-similarity model captures moderately well the dynamics of the $\tau^{\mathrm{u} *}$ contribution to the energy cascade it struggles significantly with rest of the diagnostics, especially the non-local transport. This indicates that the model represents poorly SGS diffusion processes. Interestingly, the correlations to the energy diagnostics are consistently better than the ones to the cross-helicity ones. This suggests that additionally the model is neglecting an important part of the SGS 


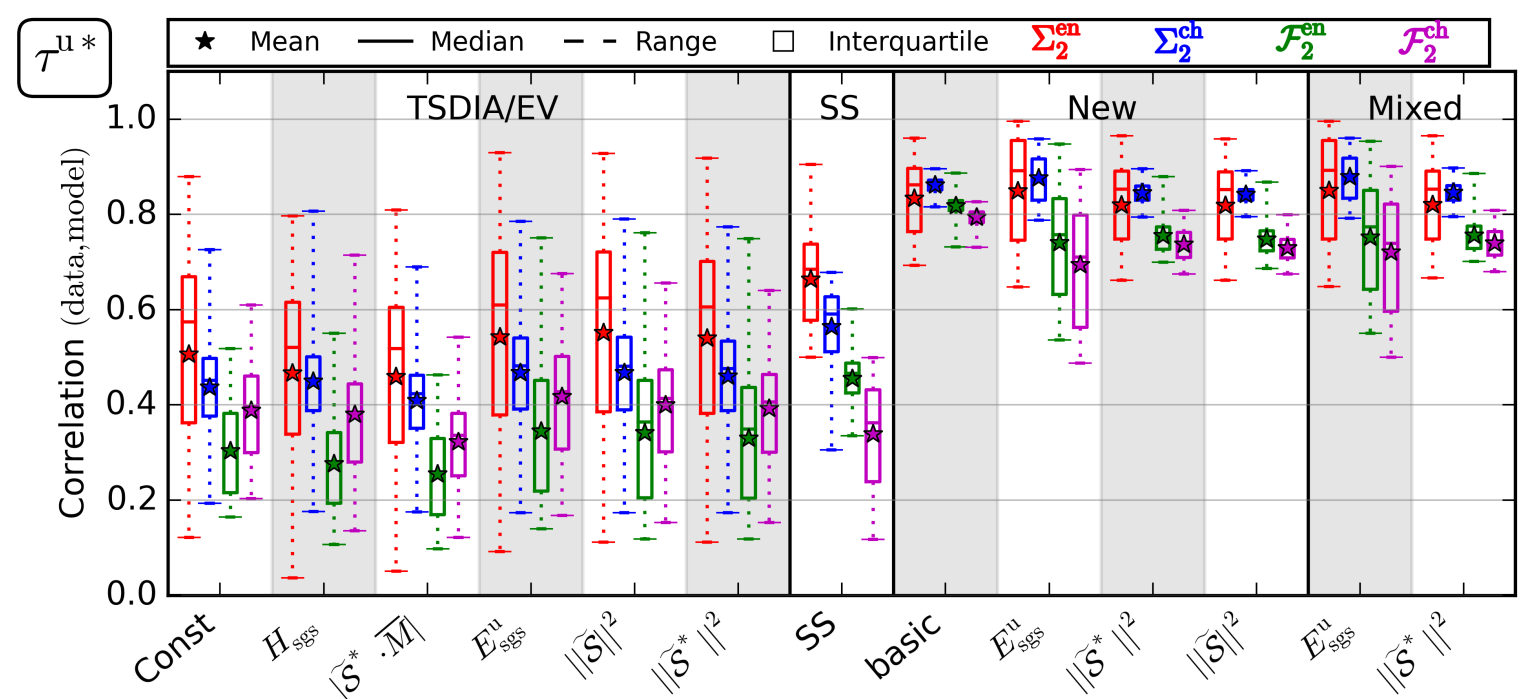

Figure 4.3: Correlation between data and model of the contributions of the term $\tau^{\mathrm{u} *}$ to the four diagnostic fields $\Sigma_{2}^{\text {en }}$ (red), $\Sigma_{2}^{\text {ch }}$ (blue), $\mathcal{F}_{2}^{\text {en }}$ (green) and $\mathcal{F}_{2}^{\text {ch }}$ (magenta). The models are grouped by families (TSDIA/EV, SS, new and mixed) and labelled within the family by the respective distinguishing modulator.

dynamics related to the kinetic-magnetic interaction.

The newly proposed model exhibits the best correlations with tightest distributions. Importantly, the re-normalisation has a detrimental effect. Although the mean and median correlations to the cascade fields are maintained, the variation is increased. There is a suggestion of the same separation between energy and cross-helicity performance as in the SS model, especially in the $E_{\mathrm{sgs}}^{\mathrm{u}}$ form. We note that this form of the model significantly increases the spread in the correlation and improves the overall fit only for some snapshots. It maintains or slightly improves the average and median correlations, but overall the total flux is represented more poorly. Remarkably, renormalizing with an energy closure seems to give better smaller variations than using the true energy. Thus, if such a step is required a posteriori for stability or other computational reasons, either of the two energy closures would be a preferable choice.

The renormalized forms also show poorer correlations for the total flux diagnostics. Therefore, the renormalization cannot be used as a substitute for the commutator between Favre filtering and differentiation for this closure. Nevertheless renormalizing with the approximate energies mitigates some of the deterioration of the $E_{\mathrm{sgs}}^{\mathrm{u}}$ model. This suggests that the Smagorinsky and realizable energies are somewhat more closely related with the commutator than the true energy. Analytically, the commutator for the total kinetic SGS stress is given by

$$
\widehat{\partial_{i} \tau_{i j}^{\mathrm{u}}}-\partial_{i} \widehat{\tau}_{i j}^{\mathrm{u}}=2 a \bar{\rho}\left(\tilde{u}_{i} \tilde{u}_{j, l}+\tilde{u}_{j} \tilde{u}_{i, l}\right) y_{, i l} .
$$

The form for deviatoric part can be extracted trivially. From this we see that a part of the commutator is related to the resolved rate-of-strain, e.g. consider $\tilde{\mathcal{S}}_{i l}=0.5\left(\tilde{u}_{i, l}+\right.$ 
$\left.\tilde{u}_{l, i}\right)$.

The mixed models do not improve the overall behaviour of the corresponding new model significantly. This is explained simply by the fact that during the fitting the corresponding EV coefficient is driven to negligence rendering the mixed models effectively equivalent to the corresponding new ones. Thus we omit these mixed models from subsequent consideration.

Examining the values of the coefficients of the non-mixed models in fig. 4.4 we observe that for the TSDIA/EV models they range over more than an order of magnitude for $\Sigma_{2}^{\text {en }}$. In other words the best correlation comes at the price of non-constant (with respect to time and plasma parameters) model coefficient. Therefore we confirm that in compressible MHD, as in hydrodynamics, a dynamic re-calculation of the coefficient is necessary for the TSDIA/EV closures of $\tau^{\mathrm{u} *}$ to reach their peak efficiency.

The scale similarity model suffers from the same problem with regards to the crosshelicity fitting, while the coefficients in the energy fits vary only by a factor of order unity. However, the mean coefficients differ substantially between the energy and crosshelicity fits. Thus in applications, there will be a significant trade-off between modelling one quantity or the other.

The new models exhibit the most stable coefficients, which also vary by a factor of order unity throughout all snapshots and all fitted fields. Remarkably, the basic model has a coefficient $\sim 1$. This implies that the modelling errors are orthogonal to the model. In other words, the effects captured by the model are captured fully, at their fundamental amplitude. This, together with small range of variation across the dataset, explains why the re-normalised versions of the model perform more poorly. Note that the coefficients of the $\|\tilde{\mathcal{S}}\|$ and $\left\|\tilde{\mathcal{S}}^{*}\right\|$ forms are systematically larger than the coefficient of the basic form. This effectively compensates for the fact that the renormalization factor is always less than unity

$$
\left\|\tilde{\mathcal{S}}^{(*)}\right\|^{2} \leq\|\nabla \tilde{\mathbf{u}}\|^{2}=\left\|\tilde{\mathcal{S}}^{*}+\nabla \cdot(\tilde{\mathbf{u}}) \mathrm{I}+\widetilde{\Omega}\right\|^{2}=\left\|\tilde{\mathcal{S}}^{*}\right\|^{2}+(\nabla \cdot \tilde{\mathbf{u}})^{2}+\|\widetilde{\Omega}\|^{2}
$$

(where the resolved vorticity tensor is given by $\left.\widetilde{\Omega}_{i j}=0.5 \epsilon_{i j k}(\tilde{\Omega})_{k}\right)$. Analogously from the coefficient of the $E_{\mathrm{sgs}}^{\mathrm{u}}$ renormalization of the model we infer that the SGS kinetic energy is of the order of the $L_{2}$-norm of the basic model. Thus, at least for the saturated regime, we don't expect the basic model to severely over-predict the amount of SGS energy and to lead globally to spurious instabilities.

Note that the three model families have different intrinsic normalisation so the absolute values of their coefficients should not be compared directly.

Next, we select a representative from each of the model families to analyse in further detail. From the TSDIA/EV group we take the energy-based eddy viscosity since it reaches the highest total-flux correlations. Specifically we choose the $E_{\mathrm{sgs}}^{\mathrm{u}}$ model as it is closest to the original EV model assumptions. From the new models we take the basic form for analogous reasons. The scale similarity model is also considered and, as discussed above, the mixed models are not.

Figure 4.5 shows a different view of the contributions of the data and the selected models with respect to the diagnostics fields. In particular, we plot similarly to chapter 3 a 


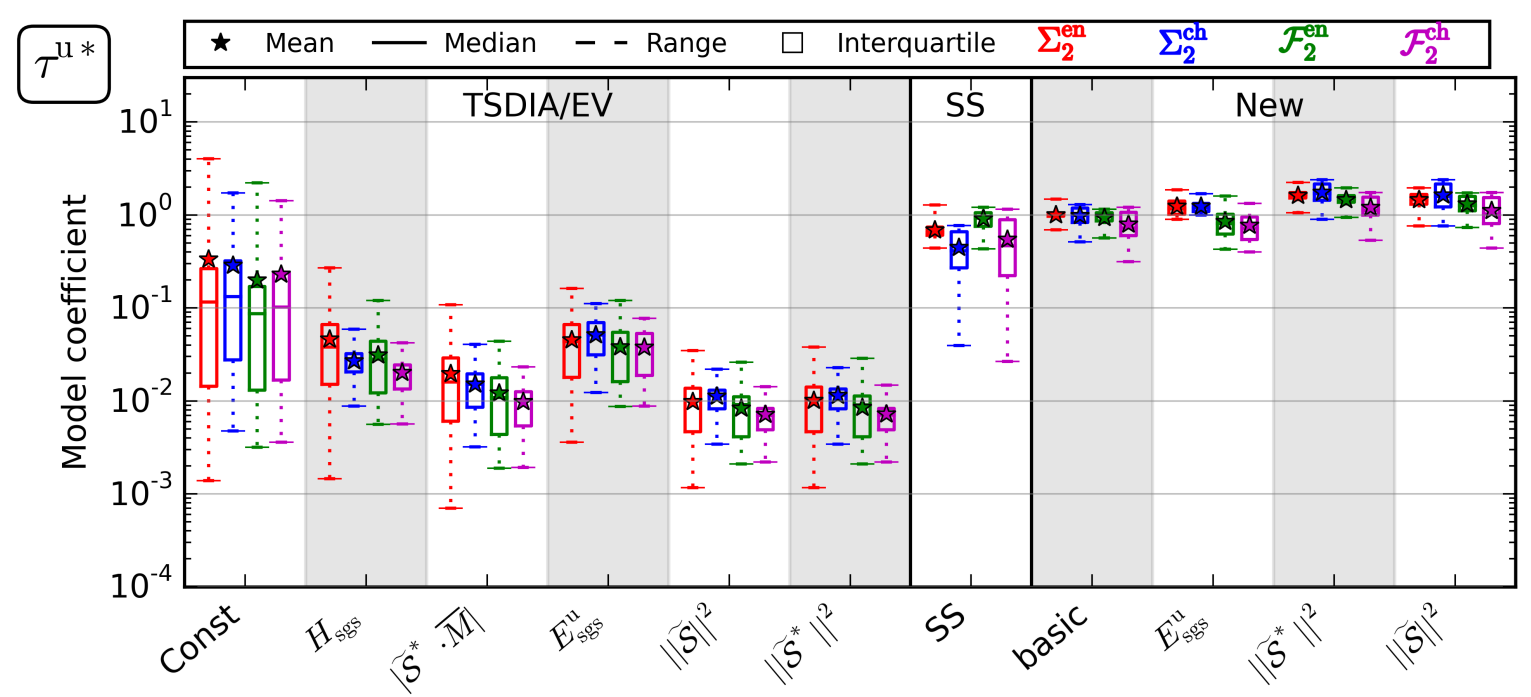

Figure 4.4: Summary of coefficient values obtained by fitting the contributions of $\tau^{\mathrm{u} *}$ to the four diagnostic fields $\Sigma_{2}^{\text {en }}$ (red), $\Sigma_{2}^{\text {ch }}$ (blue), $\mathcal{F}_{2}^{\text {en }}$ (green) and $\mathcal{F}_{2}^{\text {ch }}$ (magenta) for the new models. The symbols are the same as in fig. 4.3.

summary of the flux distribution over space and time. For each diagnostic field and for each simulation we show the median, the interquartile and the total range of the $\tau^{\mathrm{u} *}$ flux distribution in the simulation domain. Since the distributions are bimodal the median and interquartile are taken separately with respect to the positive and negative branches. In this plot positive values correspond to a forward cascade, i.e. energy transfer from large to small scales. The results are not normalised, as this is not necessary for the comparison between data and models. Note that this provides a global counterpart to the local correlation view in fig. 4.3. On the one hand two distributions over the simulation domain can present similar features while locally there is little correlation between the quantities they represent. Since the reverse does not hold, this presents a weaker constraint than the local correlation. On the other hand, the distribution contains statistical information about the strength and characteristics of the up- and down-scale transfers which is absent in the local correlation plot. Thus the two plots provide complimentary views on the flux diagnostics. Let us proceed by examining the distributions of the $\tau^{\mathrm{u} *}$ closures for each of the models and diagnostic fields.

Considering fig. 4.3 as expected the strength of the flux increases with the strength of the driving for each diagnostic field for the data and for all models. Surprisingly, there is much better correspondence between all models and the data for the supersonic simulations than the sub- and trans-sonic ones.

Considering the individual models, clearly the main reason for the poor correlations of the TSDIA/EV $E_{\mathrm{sgs}}^{\mathrm{u}}$ model is the lack of inverse cascade. As we showed in section 2.3.4, the TSDIA/EV models can only follow one part of the energy cascade $\Sigma_{2}^{\text {en }}$ at a time. For the examined dataset the fitting procedure prefers a positive coefficient, since the data has stronger forward cascade. Thus the model follows only the positive branch of 


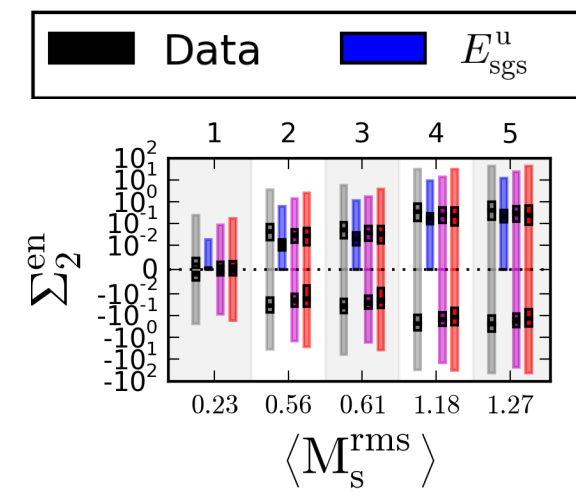

(a)

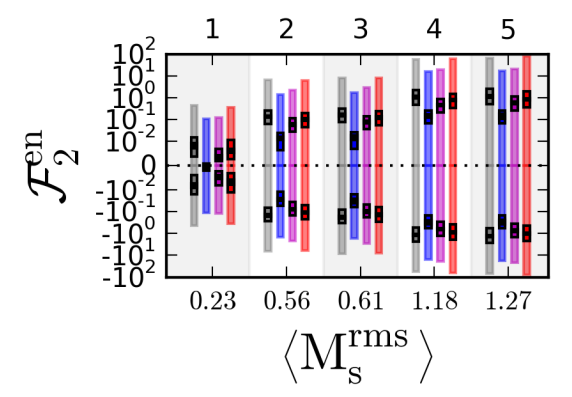

(c)

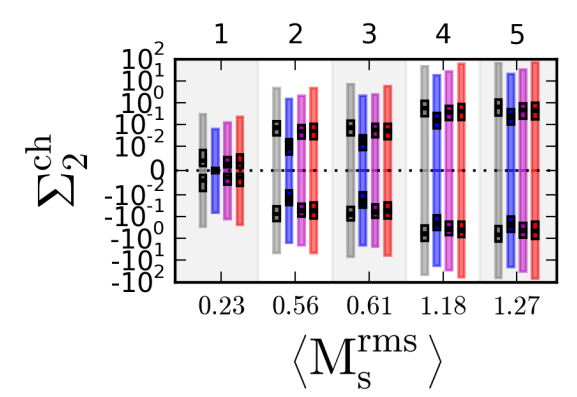

(e)

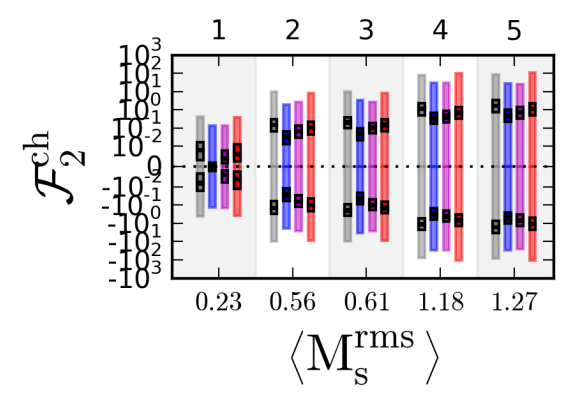

(g)

\section{SS $\square$ basic}

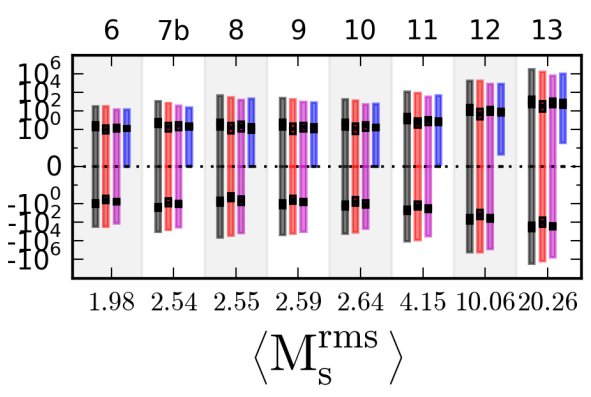

(b)

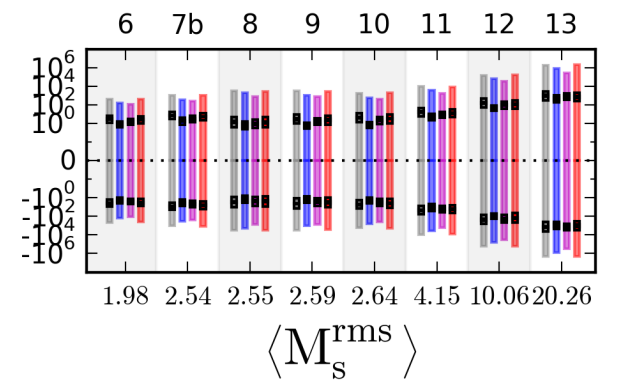

(d)

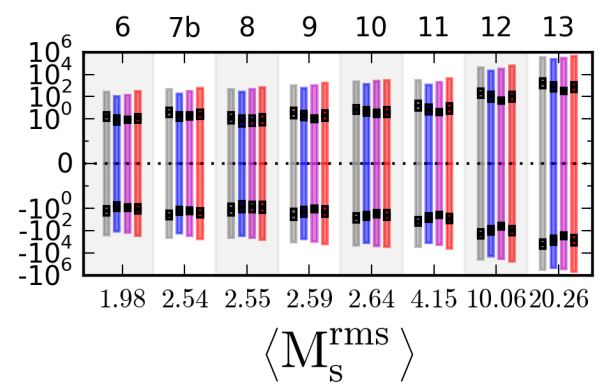

(f)

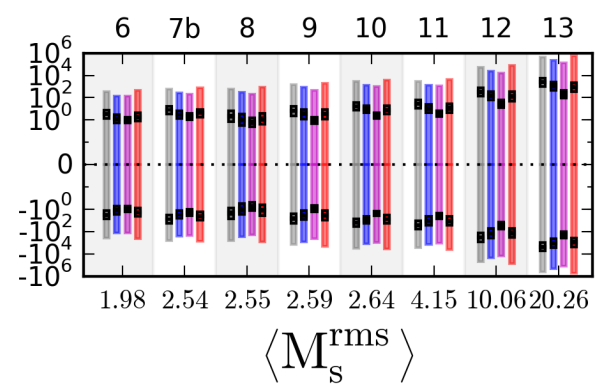

(h)

Figure 4.5: Summary of the flux distribution for the models of $\tau^{\mathrm{u} *}$ and the data: left column - sub- and trans-sonic simulations, right column - super-sonic simulations. Each row shows the contributions to one of the four diagnostic fields. The solid black rectangle represents the time variation of the median of each of the two branches of the distribution (positive down-scale transfer, negative - up-scale transfer). The empty black rectangle surrounding it shows the maximum (temporal) extent of the respective interquartile. The coloured bar shows the maximum (temporal) extent of the entire distribution. 


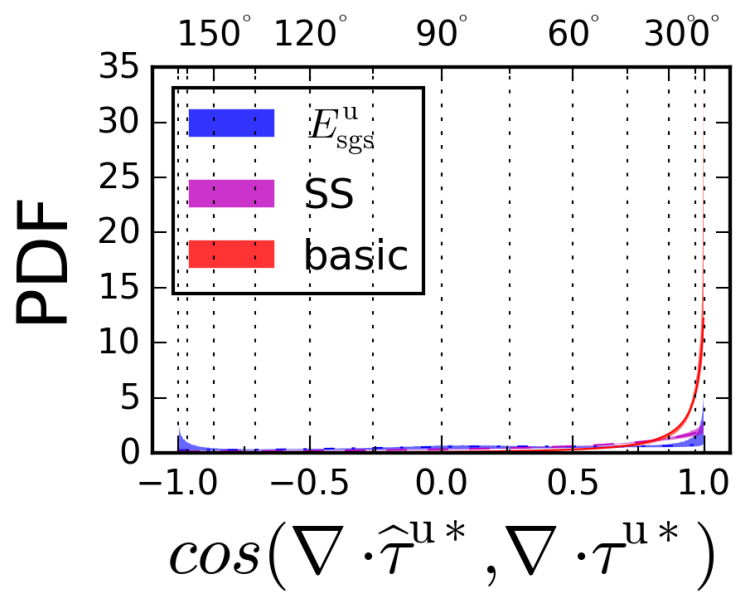

(a)

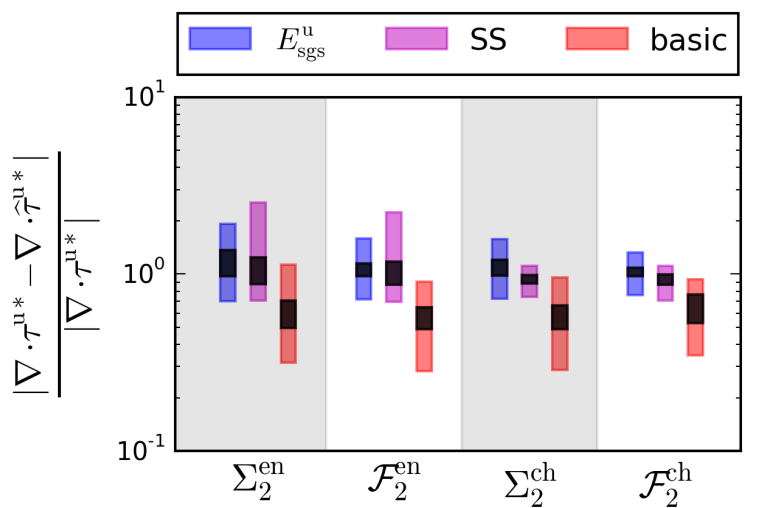

(b)

Figure 4.6: Geometry of the error vector of $\nabla \cdot\left(\tau^{\mathrm{u}}\right)$ for the TSDIA/EV $E_{\mathrm{sgs}}^{\mathrm{u}}$ (blue), the SS (purple) and the basic new (red) models. Left panel: alignment angles between data and model. The shaded area represents the variation across the entire dataset. The line represents the respective median. Right panel: size of the error vector normalised by the size of the data vector with coefficients fitted to each of the diagnostic fields. The black rectangle represents the variation across the entire dataset of the median of the distribution from each snapshot. The coloured rectangle represents the maximum extent across the entire dataset of the respective interquartile.

the transfer. Additionally, for sub-sonic simulations the TSDIA/EV model distribution predicts cascade which is weaker than the data by an order of magnitude. With the exception of these two features, the ranges and the bulk ${ }^{4}$ of the distributions of all models are relatively close to each other and to the data. Examining the remaining diagnostic fields, we note an overall correspondence between the local correlation on the one hand and how closely the bulks of the model distributions follow the bulk of the data on the other. For example, for the $\mathcal{F}_{2}^{\text {ch }}$ diagnostic the SS model presents lower correlations than the TSDIA/EV model. Accordingly for most of the simulations the bulk of SS distribution is further away from the data than the TSDIA/EV distribution is. The situation is reversed for the $\mathcal{F}_{2}^{\text {en }}$ diagnostic. Note, however that this is much less precise than the local correlation measure. Comparing the basic and SS models, the difference in their distributions is much smaller than the difference in their correlations.

We continue with a structural investigation of the models. Specifically we consider the geometry of the error between data and models at the vector level. This is given by the size of the error vector relative to the data

$$
\frac{\left|\nabla \cdot \tau^{\mathrm{u} *}-\nabla \cdot \widehat{\tau}^{\mathrm{u}}\right|}{\left|\nabla \cdot \tau^{\mathrm{u}}\right|}
$$

\footnotetext{
${ }^{4}$ more specifically the maximum temporal extent of the interquartile
} 
and the error angle given by

$$
\cos \left(\nabla \cdot \tau^{\mathrm{u} *}, \nabla \cdot \widehat{\tau}^{\mathrm{u} *}\right)=\frac{\left(\nabla \cdot \tau^{\mathrm{u} *}\right) \cdot\left(\nabla \cdot \widehat{\tau}^{\mathrm{u} *}\right)}{\left|\nabla \cdot \tau^{\mathrm{u} *}\right|\left|\nabla \cdot \widehat{\tau}^{\mathrm{u} *}\right|} .
$$

Figure 4.6 shows these quantities summarized across all diagnostic fields and over the entire dataset. We begin with a consideration of the error angle. As in the previous chapter, the variation over time and plasma parameter is practically negligible. Since we are considering one-coefficient models, the angle is in fact between $\nabla \cdot \tau^{\mathrm{u} *}$ and a set of large-scale vector fields. As we noted in the previous chapter, this is a robust structural feature of the examined dataset. More importantly, it is not affected by the different fitting criteria, since they only change the coefficient and hence the size of the model vector, but not its direction. Therefore all diagnostic fits are degenerate for this plot. Moreover, the results for the TSDIA/EV $E_{\mathrm{sgs}}^{\mathrm{u}}$ model apply to the whole family, since the different models there differ only in their magnitude.

Figure 4.6a shows one of the key structural features of the new model - it can capture the orientation of $\nabla \cdot \tau^{\mathrm{u} *}$ with very good accuracy. The error angle distribution peaks at $0^{\circ}$ with $56.8 \%$ of the dataset within $30^{\circ}$ of the data. It should be noted that this accuracy may be improved further pending the commutator correction discussed above. Even in comparison to this form the other two models are insensitive to the orientation of the data - the TSDIA/EV model has $9.4 \%$ and the SS model has $22 \%$ of the dataset within $30^{\circ}$ of the data.

Next consider the relative size of the error-vector. Intuitively, it is one of the first quantities on which structural comparisons can be based. However, its interpretation need to be handled with care as it is a compound quantity which carries information about the relative size of the model as well as its orientation. For instance, a model vector with the correct size which is anti-parallel to the data vector would produce an error vector with the same size as a correctly oriented model which is three time too large. A priori it is not established which of the scenarios is more favourable in an $a$ posteriori LES application, or even if there is a unique answer to that question. This issue is exacerbated by the fact that the error vector size depends on the value of the model coefficients and thus changes across the fits to the different diagnostic fields.

Nevertheless, consider fig. 4.6b. It shows an aggregate from the entire dataset of the relative error vector size for each model with coefficients fitted to each of the diagnostic fields. The primary observation here is that all models produce error vectors of size comparable to the data independent of the diagnostic field. Both the TSDIA/EV and the SS models produce error vectors with relative size of order unity or larger, while the new model has a median which ranges between 0.5 and 0.8 . For the TSDIA/EV and the SS models both the variations and the median are smaller for the cross-helicity diagnostics compared to the energy ones. This dichotomy is not present for the new model which is also relatively stable across diagnostic fields. A comparison of these observations with fig. 4.4 indicates that the relative error vector size is not sensitive to the local dynamic correlation between data and model evaluated via the diagnostic fields. Combining this result with the ones drawn from fig. 4.6a, we conclude that for $\tau^{\mathrm{u} *}$ the most dynamically relevant feature of the deviatoric kinetic SGS stress at the vector level is its orientation, while its amplitude has a secondary role. Before 

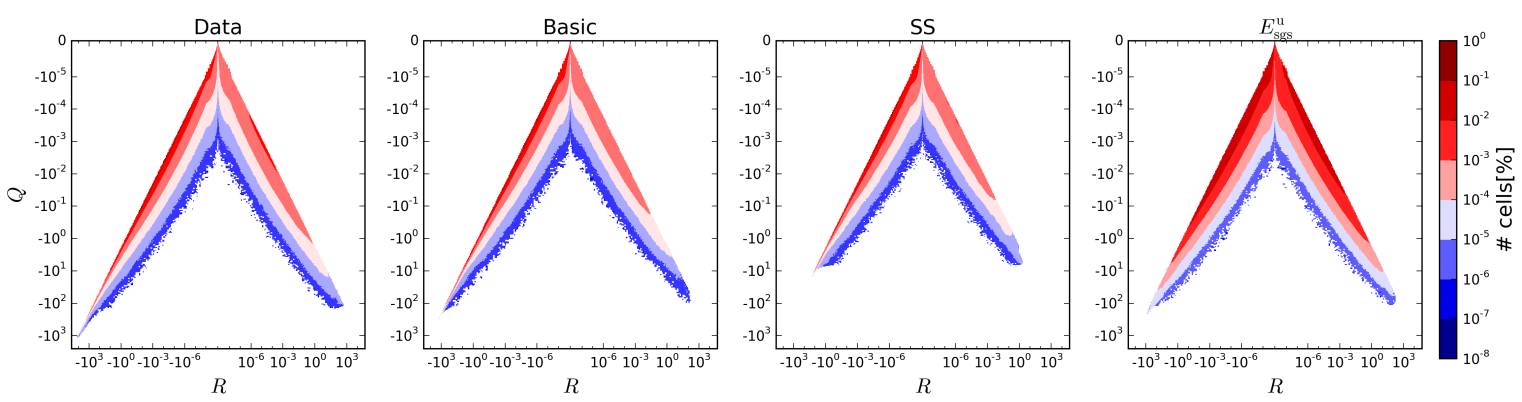

Figure 4.7: The $Q-R$ portrait of $\tau^{\mathrm{u} *}$ for the data and selected models of a sample snapshot from simulation 9 with $\mathrm{M}_{\mathrm{s}, \mathrm{dr}}=2.9$ and $\beta_{\mathrm{p}, 0}=2.5$. The values are not normalised. The contours indicate the percentage of the cells with respect to the whole simulation domain.
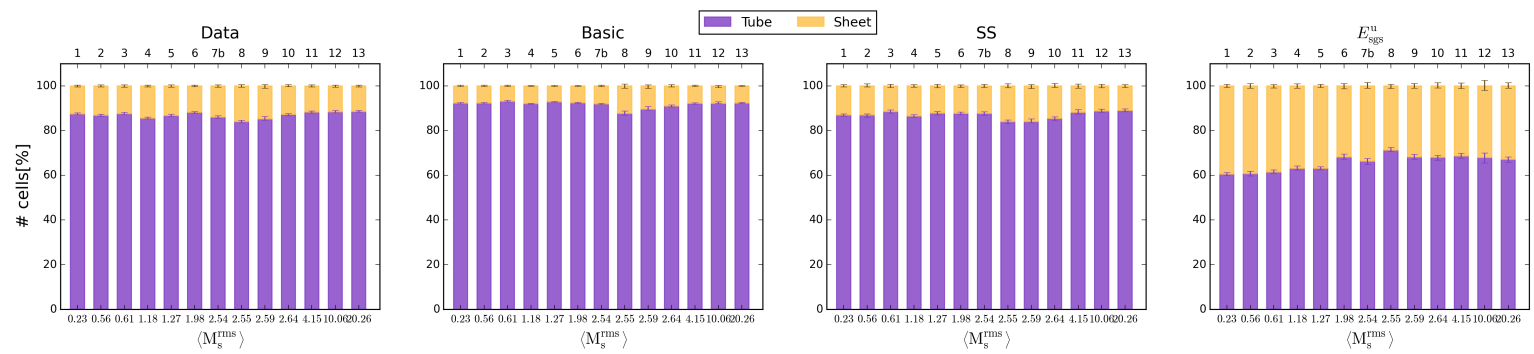

Figure 4.8: Summary of the distribution between the tube and sheet topologies for $\tau^{\mathrm{u} *}$ for all simulations. Error bars represent variations within the time interval between $2 T$ and $5 T$.

we examine whether this statement holds for the other SGS terms, we conclude the comparison of the deviatoric kinetic models with a direct tensor comparison.

At the tensor level we established the Q-R portrait of $\tau^{\mathrm{u} *}$ as a robust feature of the SGS dynamics. Note that it is also not affected by coefficient values for single coefficient models. Figure 4.7 shows a representative snapshot (from simulation 9 with $\mathrm{M}_{\mathrm{s}, \mathrm{dr}}=2.9$ and $\beta_{\mathrm{p}, 0}=2.5$ ) of this portrait for the data and the different models. Although all models have qualitatively the same distribution, the main difference between them is the range of values that both the $Q$ and the $R$ parameters can take. The new model is the only one which reaches with non-negligible frequency the same orders of magnitude $\left(Q \sim-10^{2}, R \sim-10^{3}\right)$ in the dominant topological region as the data. This suggests that a key property for a $\tau^{\mathrm{u} *}$ model is the ability to reach the far $Q-R$ tails of the turbulent distribution.

From a global perspective, we consider the data's and models' distribution between the two topological regions (tube and sheet). Figure 4.8 summarizes the results for the different simulations. The data and all models show approximately constant ratio (with respect to time) between sheet and tube topologies for all simulations. As we noted in section 3.3.1 the tube topology dominates in the data. The new models mimics this behaviour and to a lesser extent so does the scale-similarity model. However, in the TSDIA/EV model the ratio is significantly reduced. This is consistent with the dynamic flux correlations established in the beginning of this section. Thus it supports empirically the link between the eigenvalues of $\tau^{\mathrm{u} *}$ and the diagnostic flux terms, which 


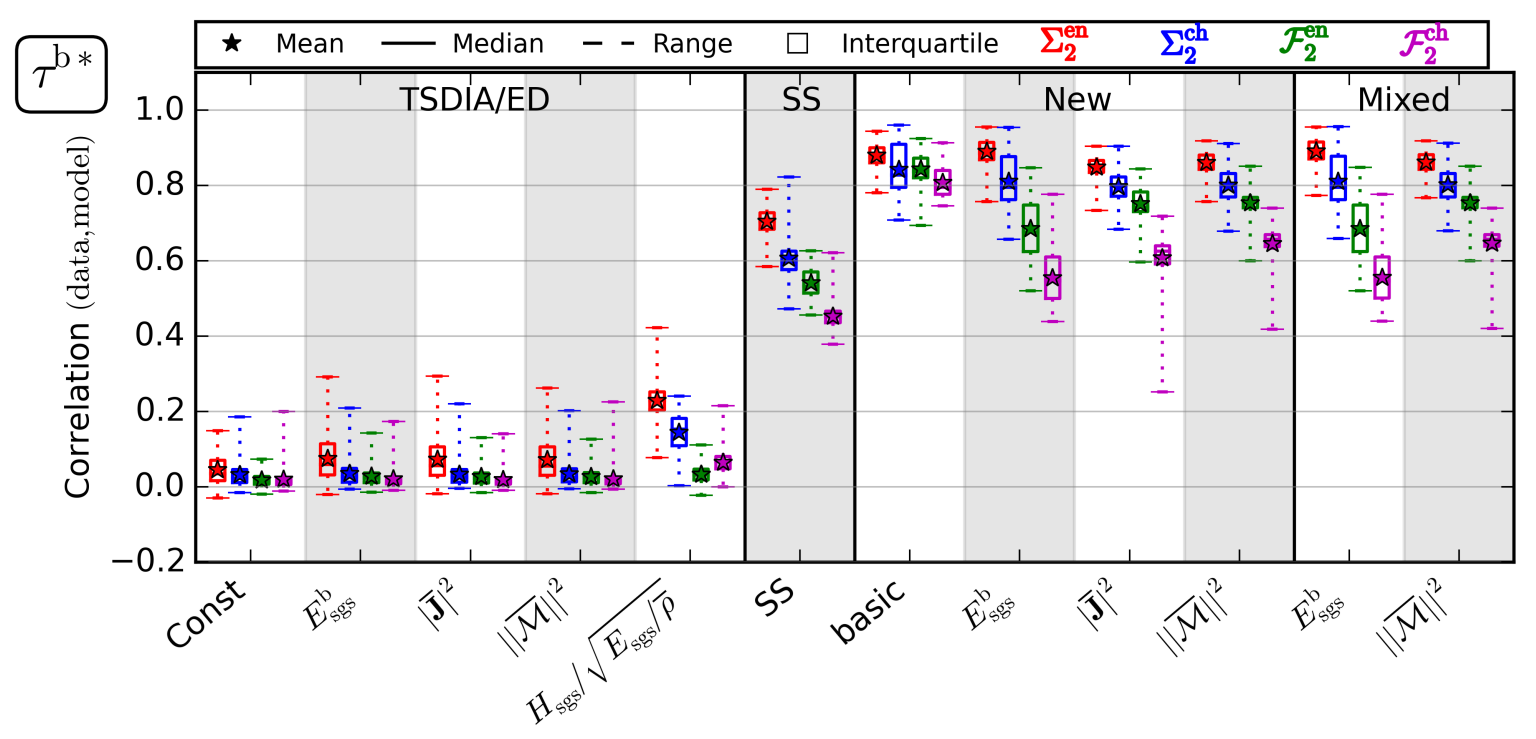

Figure 4.9: Correlation between data and model of the contributions of the term $\tau^{\mathrm{b} *}$ to the four diagnostic fields $\Sigma_{2}^{\text {en }}$ (red), $\Sigma_{2}^{\text {ch }}$ (blue), $\mathcal{F}_{2}^{\text {en }}$ (green) and $\mathcal{F}_{2}^{\text {ch }}$ (magenta). The models are grouped by families (TSDIA/ED, SS, new and mixed) and labelled within the family by the characteristic modulator.

we tentatively established in section 2.3.4. The further analysis required to fully explore this connection is left as part of future investigations. However already at this point we can conclude that the tensor invariants are strongly connected to the SGS dynamics of $\tau^{\mathrm{u} *}$.

\subsection{Deviatoric magnetic SGS stress: $\tau^{\text {b* }}$}

As for the kinetic stress, the closures of $\tau^{\mathrm{b} *}$ are also separated into 4 families - TSDIA/ED, SS, new and mixed. The TSDIA/ED closures are characterised by the form $\tau^{\mathrm{b} *} \sim \nu_{\mathrm{b}} \overline{\mathcal{M}}$ and are labelled by the characteristic field which determines the eddydiffusivity. The new models are the basic and the renormalized ones, where the renormalization is done via the real magnetic SGS energy or one of its closures. Finally, the mixed closures are two-coefficient linear combinations of the new and TSDIA/ED closures scaled by the true or the Smagorinsky SGS magnetic energy. The performance of all the models with regards to the four diagnostic fields is presented in fig. 4.9.

The first striking observation is that the TSDIA/ED closures are extremely unfit to represent the data. There are isolated cases where the correlation coefficient even changes sign. The eddy-diffusivity based on cross-helicity seems to exhibit the best performance with correlation to $\Sigma_{2}^{\text {en }}$ reaching 0.4 in isolated snapshots. However these are exceptional cases. The total flux diagnostics show basically no correlation with the data. Based on this diagnostic alone this family of models should be disregarded altogether.

For the rest of the models, with the exception of the new basic one, features familiar 
from the $\tau^{\mathrm{u} *}$ investigation are evident. The energy correlations are in general larger than the cross-helicity ones and cascade terms are more accurately represented than total flux terms. For the scale-similarity model the correlations are actually higher and their variation smaller than the corresponding $\tau^{\mathrm{u} *}$ results. The mean and median correlations are also higher for the new basic model. The reason for the synchronized improvement in both models can be attributed to the fact that the $\tau^{\mathrm{b} *}$ modelling process is not affected by the Favre filtering-differentiation commutator issue. The still strong gradation of correlation from cascade to full flux and from energy to crosshelicity diagnostics of the scale-similarity model suggests that also for $\tau^{\text {b* }}$ it misses a significant component of the SGS Lorentz/magnetic dynamics related to diffusion and the kinetic-magnetic interaction.

The basic form of the new model has the highest core correlations. The smaller deterioration in the total flux fits with respect to the cascade ones indicates that the same deterioration in the basic kinetic model is indeed due to the Favre filteringdifferentiation commutator. In comparison to their kinetic counterparts, the $\Sigma_{2}^{\text {en }}$ fits of all new model forms provide a little better correlations with much smaller core variations (as measured by the length of the interquartile). On the other hand, with the exception of the $E_{\mathrm{sgs}}^{\mathrm{b}}$ form, the other diagnostic fields exhibit much larger overall variation (as measured by the length of the whiskers). The notably poor performance in the $\mathcal{F}_{2}^{\text {ch }}$ fits is produced by a few snapshots in the trans-sonic simulations.

For the total flux diagnostics the deterioration introduced by the renormalization (with any of the magnetic energies) is stronger than it is in the kinetic case. This implies that here the renormalization introduces an error larger than the combined commutator plus renormalization error from the kinetic case. For the basic and the $E_{\mathrm{sgs}}^{\mathrm{b}}$ models the correlations increase with $\mathrm{M}_{\mathrm{s}}$ for all diagnostics. The other two models are insensitive to the sonic Mach number, however there is a trend of $\mathcal{F}_{2}^{\text {ch }}$ correlations decreasing with decreasing $\beta_{\mathrm{p}}$ in the trans-sonic regime.

Regarding the mixed models, there is again no qualitative difference from the respective new models. The reason is the same as in the kinetic case. The coefficients of the eddydiffusivity component is about two orders of magnitude smaller than the coefficients of the new closure component. Considering the correlations of the TSDIA/ED models this is not surprising.

The coefficients of the SS and new models are shown in fig. 4.10. The overall variation is comparable to the one in the kinetic case, but the core variation is much smaller. We see that typically the SS coefficients for the energy diagnostics are significantly different (larger) from the ones for the cross-helicity diagnostics. Therefore here, as in the kinetic case, a choice has to be made as to which effects to prioritize in a posteriori implementation.

The larger coefficients of the $\|\overline{\mathcal{M}}\|^{2}$ and $|\overline{\mathbf{J}}|^{2}$ new model forms as compared to the basic one can be explained by an argument analogous to the one in the kinetic case. Furthermore, observe that the renormalization factors of the two models are the complements of each other (i.e. $\|\overline{\mathcal{M}}\|^{2}+|\overline{\mathbf{J}}|^{2}=\|\nabla \overline{\mathbf{B}}\|^{2}$ ). Therefore, since the resolved current is much larger than the resolved magnetic rate-of-strain (not shown), we see that the $|\overline{\mathbf{J}}|^{2}$ coefficient is much closer to the basic one and also significantly smaller than the $\|\overline{\mathcal{M}}\|^{2}$ ones. The $E_{\text {sgs }}^{\mathrm{b}}$ coefficients differ from the basic ones again by a small factor. 


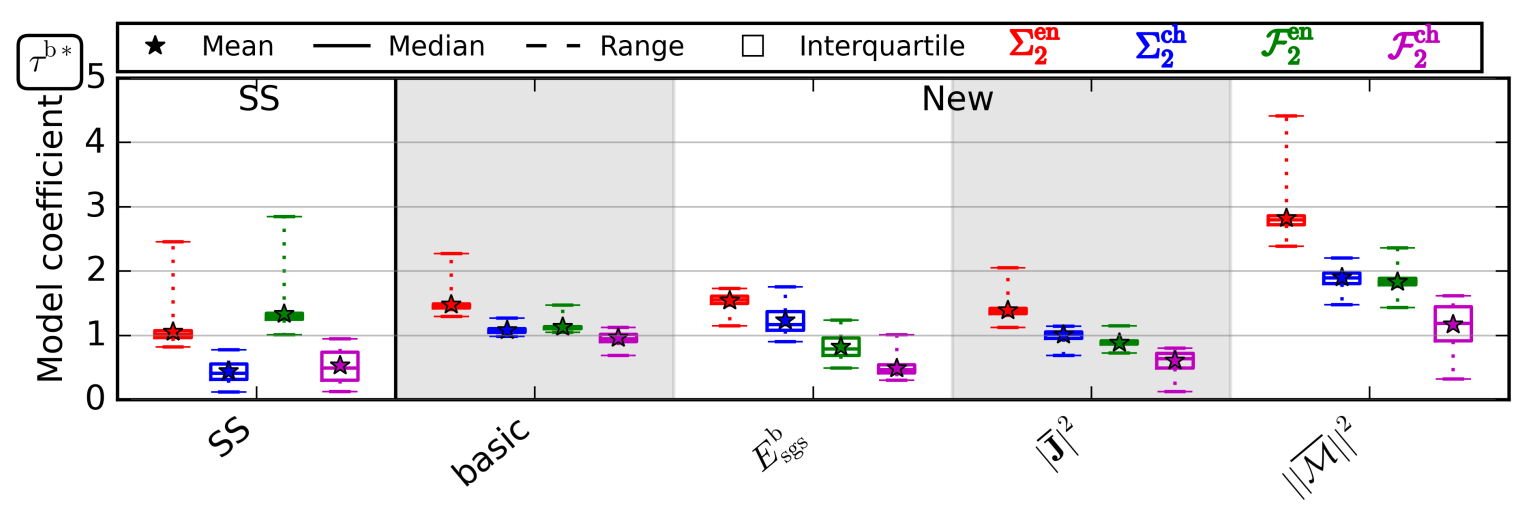

Figure 4.10: Summary of coefficient values obtained by fitting the contributions of the term $\tau^{\mathrm{b} *}$ to the four diagnostic fields $\Sigma_{2}^{\mathrm{en}}$ (red), $\Sigma_{2}^{\text {ch }}$ (blue), $\mathcal{F}_{2}^{\mathrm{en}}$ (green) and $\mathcal{F}_{2}^{\mathrm{ch}}$ (magenta). The models and statistical features are as in fig. 4.9.

This suggests that the basic magnetic model also has an $L_{2}$ norm comparable to the SGS magnetic energy. Therefore it should not drive globally any spurious instabilities just as in the kinetic case. The coefficients of the basic new model have very small variations in comparison to the other models. This makes it very attractive from the point of view of a posteriori applications. A comparison with the basic kinetic model coefficient variation, considering that the magnetic stress does not include Favre filtering, suggests that the slightly larger variability in the kinetic coefficient value is in part due to the compressibility effects.

Let us proceed with a more detailed view into a selection models. From the TSDIA/ED family we consider the form based on the true SGS cross-helicity, since it shows some of the better correlations within its group. From the new models we take the basic form. Thus we can consider a direct comparison between the kinetic and magnetic case. Once more we consider the scale similarity model and disregard the mixed models.

Figure 4.11 is the magnetic analogue of fig. 4.5 and is qualitatively similar to it. Even though the TSDIA/ED model can in principle represent both branches of the crossscale transfer, it significantly under-predicts its amplitude for all diagnostic fields. Like in kinetic case, the subsonic and trans-sonic simulations present the biggest challenge. Additionally here, the simulations with largely compressive forcing (sim. 8, 9, and 10) induce high variability and even complete model failure for some snapshots, as indicated by median of the $\mathcal{F}_{2}^{\text {en }}, \Sigma_{2}^{\text {ch }}$, and $\mathcal{F}_{2}^{\text {ch }}$ contributions.

The cores of the distributions of the new basic and the scale-similarity models are very similar and close to the data. However, the total extent of the basic model distribution is always larger than the SS distribution. This places it closer to the data everywhere with the exception of the $\Sigma_{2}^{c h}$ diagnostic for some of the supersonic simulations. Considering that only for the $\Sigma_{2}^{\text {ch }}$ diagnostic the SS correlation is similarly sometimes larger than the basic model one's, it is tempting to establish a tentative link between the local flux correlation and the tails of the flux distribution. However, after a re-examination of the tails of the TSDIA/ED model's distribution, such a link has to be discarded as an artefact. Specifically, for high enough agreement between model 


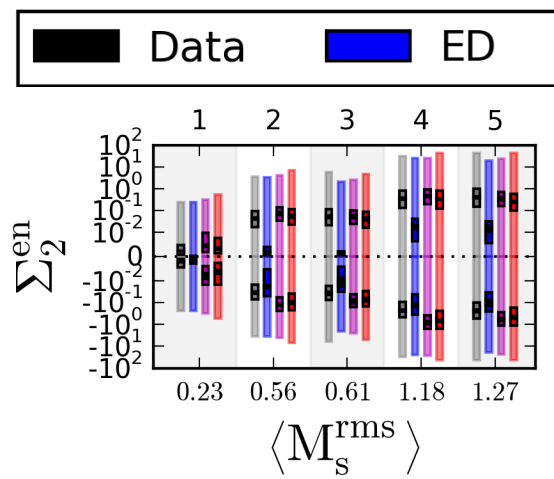

(a)

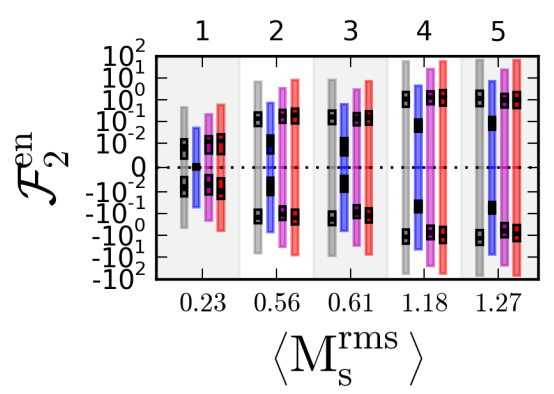

(c)

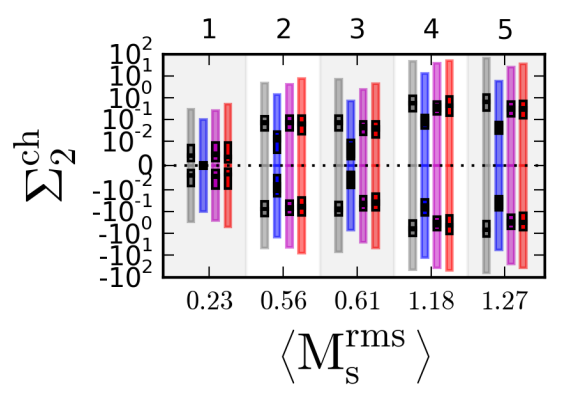

(e)

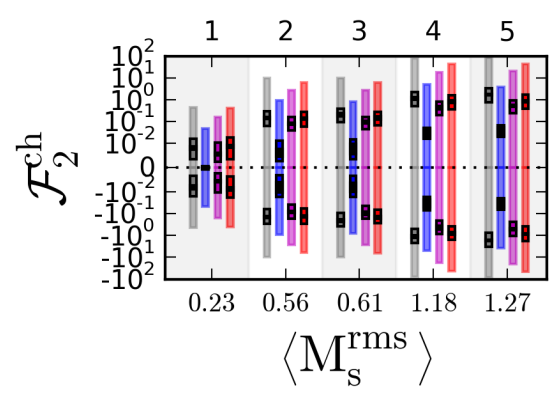

$(\mathrm{g})$

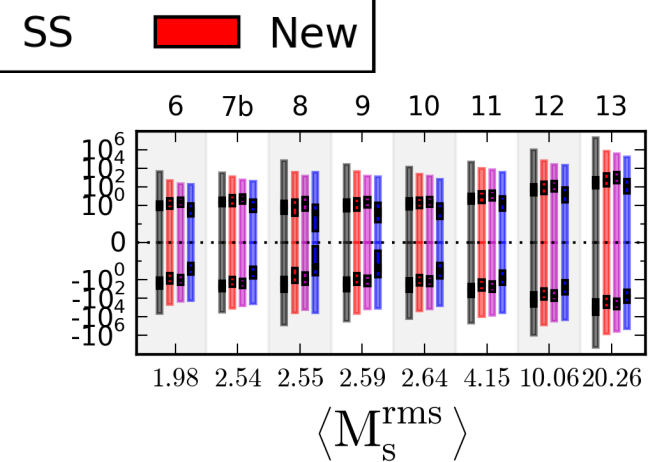

(b)

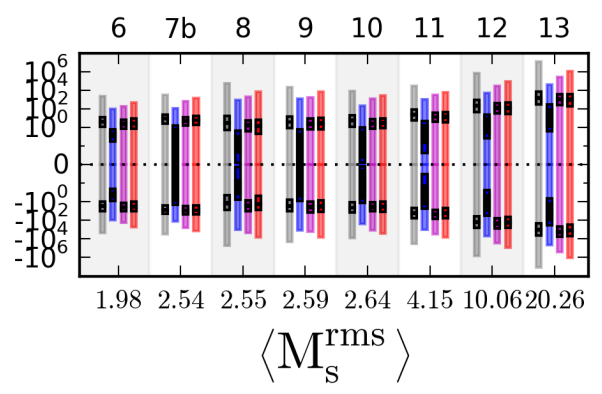

(d)

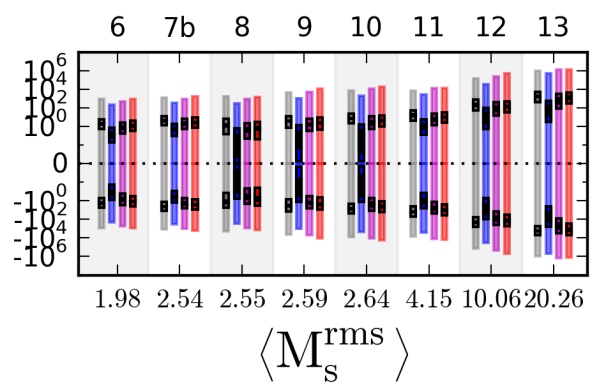

(f)

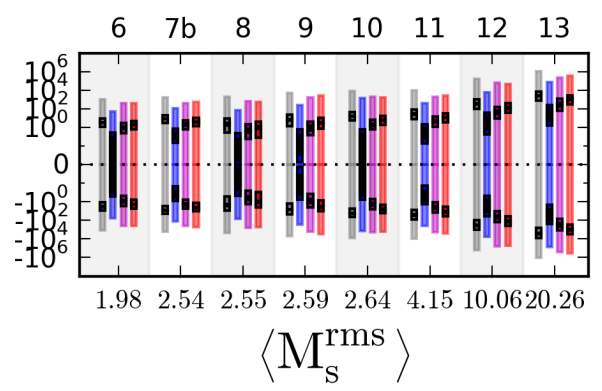

(h)

Figure 4.11: Summary of the flux distribution for the models of $\tau^{\mathrm{b} *}$ and the data: left column - sub- and trans-sonic simulations, right column - super-sonic simulations. Each row shows the contributions to one of the four diagnostic fields. The solid black rectangle represents the time variation of the median of each of the two branches of the distribution (positive down-scale transfer, negative - up-scale transfer). The empty black rectangle surrounding it shows the maximum (temporal) extent of the respective interquartile. The coloured bar shows the maximum (temporal) extent of the entire distribution. 


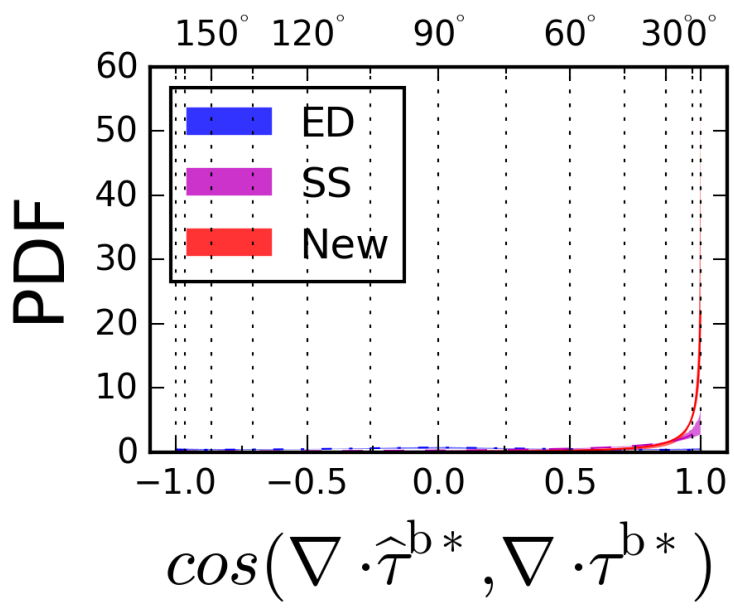

(a)

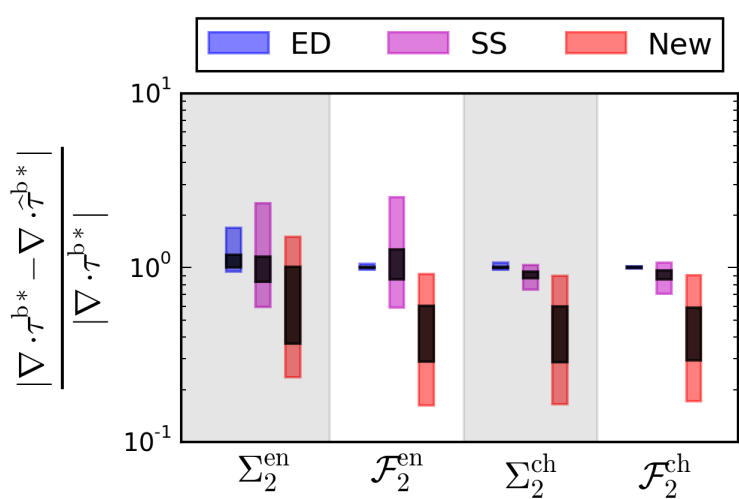

(b)

Figure 4.12: Geometry of the error vector of $\nabla \cdot\left(\tau^{\mathrm{b} *}\right)$ for the TSDIA $/ \operatorname{ED} H_{\mathrm{sgs}} / \sqrt{E_{\mathrm{sgs}} / \bar{\rho}}$ (blue), the SS (purple) and the basic new (red) models. Left panel: alignment angles between data and model. The shaded area represents the variation across the entire dataset. The line represents the respective median. Right panel: size of the error vector normalised by the size of the data vector with coefficients fitted to each of the diagnostic fields. The black rectangle represents the variation across the entire dataset of the median of the distribution from each snapshot. The coloured rectangle represents the maximum extent across the entire dataset of the respective interquartile.

and data both the local correlation and global flux distribution results coincide. As the agreement decreases, the coincidence also decreases in a fashion which depends on the particulars of the model and data distributions and varies greatly across the different snapshots.

Thus as in the kinetic case, we see that a comparison of the flux distributions can differentiate between models with qualitatively different performance and sheds light on the reasons for a models behaviour. However, it is less sensitive than the local correlation coefficient.

Let us continue with the structural comparison of the models. The error vector geometry is presented in fig. 4.12. A comparison of the error angles demonstrates that the new model has the best alignment with the data. For it $64 \%$ of the modelled points lie within $30^{\circ}$ of the data vector. This number for the TSDIA/ED model is $4 \%$ and for the SS model - 31\%. This re-enforces the connection between the error angle and the flux correlations, which we established in the previous section. Additionally, note that the alignment here is stronger than it was for the model of $\tau^{\mathrm{u} *}$. This suggests that adding the Favre filtering-differentiation commutator to the $\tau^{\mathrm{u} *}$ model should improve its alignment with the data.

The relative sizes of the error vectors also confirms the conclusions drawn in the previous section. In particular, the relative error vector size enjoys a highly non-linear nonmonotonic relationship with the flux correlations. Note that the model with lowest flux correlations exhibits here the tightest distributions, which are centred around unity. In 

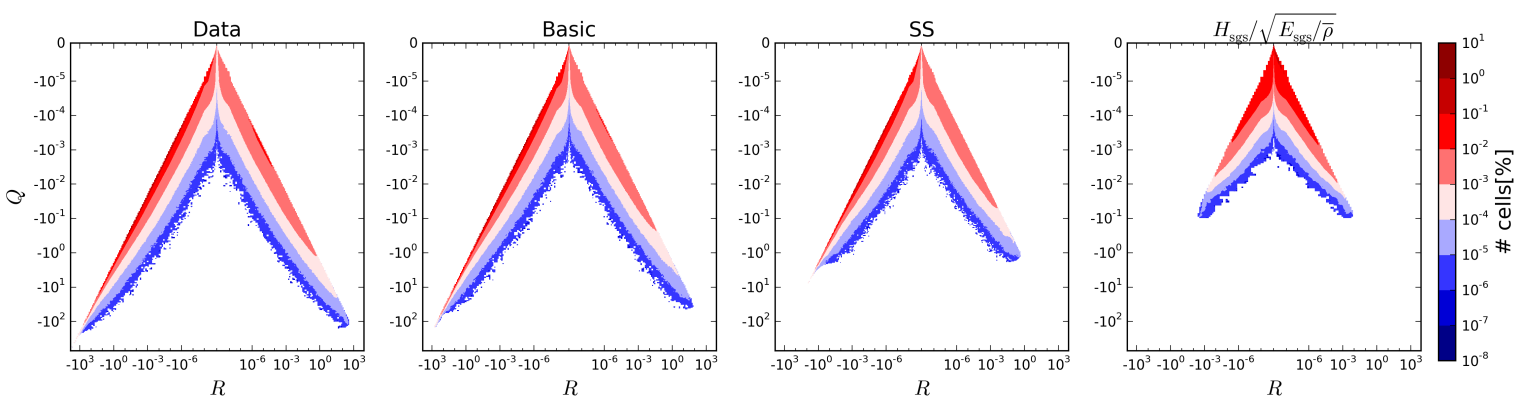

Figure 4.13: The Q-R portrait of $\tau^{\mathrm{b} *}$ for the data and selected models of a sample snapshot from simulation 9 with $\mathrm{M}_{\mathrm{s}, \mathrm{dr}}=2.9$ and $\beta_{\mathrm{p}, 0}=2.5$. The values are not normalised. The contours indicate the percentage of the cells with respect to the whole simulation domain.
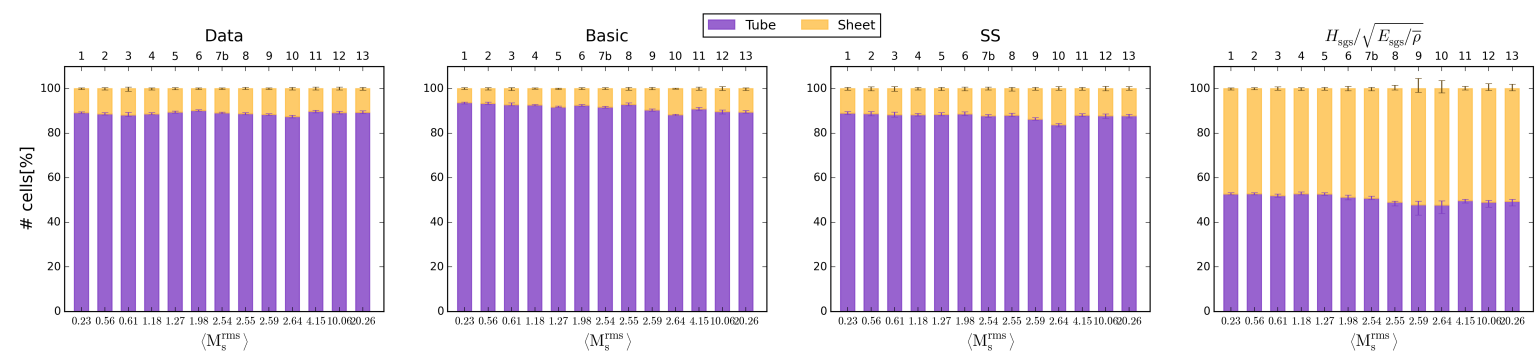

Figure 4.14: Summary of the distribution between the tube and sheet topologies for $\tau^{\mathrm{b} *}$ for all simulations. Error bars represent variations within the time interval between $2 T$ and $5 T$.

general, we observe that higher flux correlations correspond to broader distributions of the relative error-vector size and low correlations correspond to tight distributions centred around unity. The new model also follows this trend. Moreover its distribution is also shifted largely below the unity line. Additionally, its correlations for $\Sigma_{2}^{\text {en }}$ are higher than for $\mathcal{F}_{2}^{\text {en }}$, while the respective relative error vector size is also greater.

At the tensor level, we see (in fig. 4.13) a picture similar to the kinetic case. The data is dominated by tube topology and the models with better flux correlations reach the extreme $Q$ and $R$ values with higher frequency. The distribution of the data and models between the topological regions for all simulations is presented in fig. 4.14. All models show self-consistent behaviour across all simulations. At this level the signature of the TSDIA/ED model's poor performance is a significant over-estimation of the regions with sheet topology. The scale similarity and new models represent the data faithfully. However, note that the new model slightly over-estimates the tube contribution for the low Mach number simulations, while the scale similarity model is closer to the data. This indicates that the topological division, just as the flux distribution, agrees with the flux correlation for models with qualitatively different performance. However, it is less sensitive to small quantitative changes of the model. 


\subsection{Deviatoric total SGS stress: $\tau^{*}$}

We finish the discussion of the deviatoric stress models with the closures for $\tau^{*}=$ $\tau^{\mathrm{u} *}-\tau^{\mathrm{b} *}$. This demonstrates the effect of combining kinetic and magnetic closures. Do the individual modelling errors enhance or reduce one another?

We compare four model families. The TSDIA and the EV approaches lead to closures with different tensor form. The EV closures have the form $\tau^{*} \sim \nu_{\mathrm{sgs}} \tilde{\mathcal{S}}$. The TSDIA closures are 2-coefficient combinations of the form $\tau^{*}=C_{1} \nu_{\mathrm{u}} \bar{\rho} \tilde{\mathcal{S}}^{*}+C_{2} \nu_{\mathrm{b}} \overline{\mathcal{M}}$. The scale similarity and the new model versions comprise of similar linear combinations of their respective kinetic and magnetic components. Based on the arguments from the kinetic and magnetic stress tensors we omit the sets of mixed models. The flux correlations for all models are presented in fig. 4.15.

Within each family the various model flavours induce little change in the overall performance. Comparing the TSDIA and EV closures, we see that despite the added flexibility of two independent terms, the TSDIA closures do not perform significantly better. This is due to the poor correlations of the magnetic closures which we noted in the previous section. Consequently, the magnetic coefficients of the TSDIA models are two orders of magnitude smaller than their kinetic counterparts (not shown). Even so, the TSDIA correlations are consistently higher than for the kinetic stress alone. As this cannot be attributed to the magnetic closure, it suggests that the kinetic component of the TSDIA models are actually better at describing the total deviatoric stress than the kinetic one. In other words these models should be merged into the EV family in this context.

The correlations of the scale similarity model to the diagnostic fields are a combination of the features of the kinetic and magnetic components. The length of the interquartile lies between the corresponding kinetic and magnetic ones. The hierarchy between energy and cross-helicity and cascade and total flux correlations is also preserved. The overall correlation is improved with respect to the individual kinetic and magnetic ones. However, the amount of improvement is small and can be attributed to a fine tuning of the two free coefficients. Note that the energy fits benefit from this more than the crosshelicity ones. We conclude that the scale similarity model performs self-consistently with respect to the combination $\tau^{*}=\tau^{\mathrm{u} *}-\tau^{\mathrm{b} *}$.

Similarly for the new model family, a combination of kinetic and magnetic features is observed. Since the magnetic component is not manifestly dominant it cannot mask the missing Favre filtering-differentiation commutator term in the total flux fits and we note the reduced correlations in all model forms. The true energy form has the largest correlations for the $\Sigma_{2}^{\text {en }}$ diagnostic, but lags behind in the total flux diagnostics. It also leads to too much variation in the correlations and thus is removed from consideration. The Smagorinsky and realizable energy are viable alternatives for renormalization. However the basic model remains a better candidate with larger and tighter correlations for all diagnostic fields

For the detailed comparison we select three models: the true-energy-based TSDIA model labelled as " $E_{\mathrm{sgs}}^{\mathrm{u}} ; E_{\mathrm{sgs}}^{\mathrm{b}}$ ", the scale similarity model and the new basic model. Formally they have the same number of degrees of freedom which allows for a direct comparison. Furthermore, as we noted the TSDIA models can faithfully represent also 


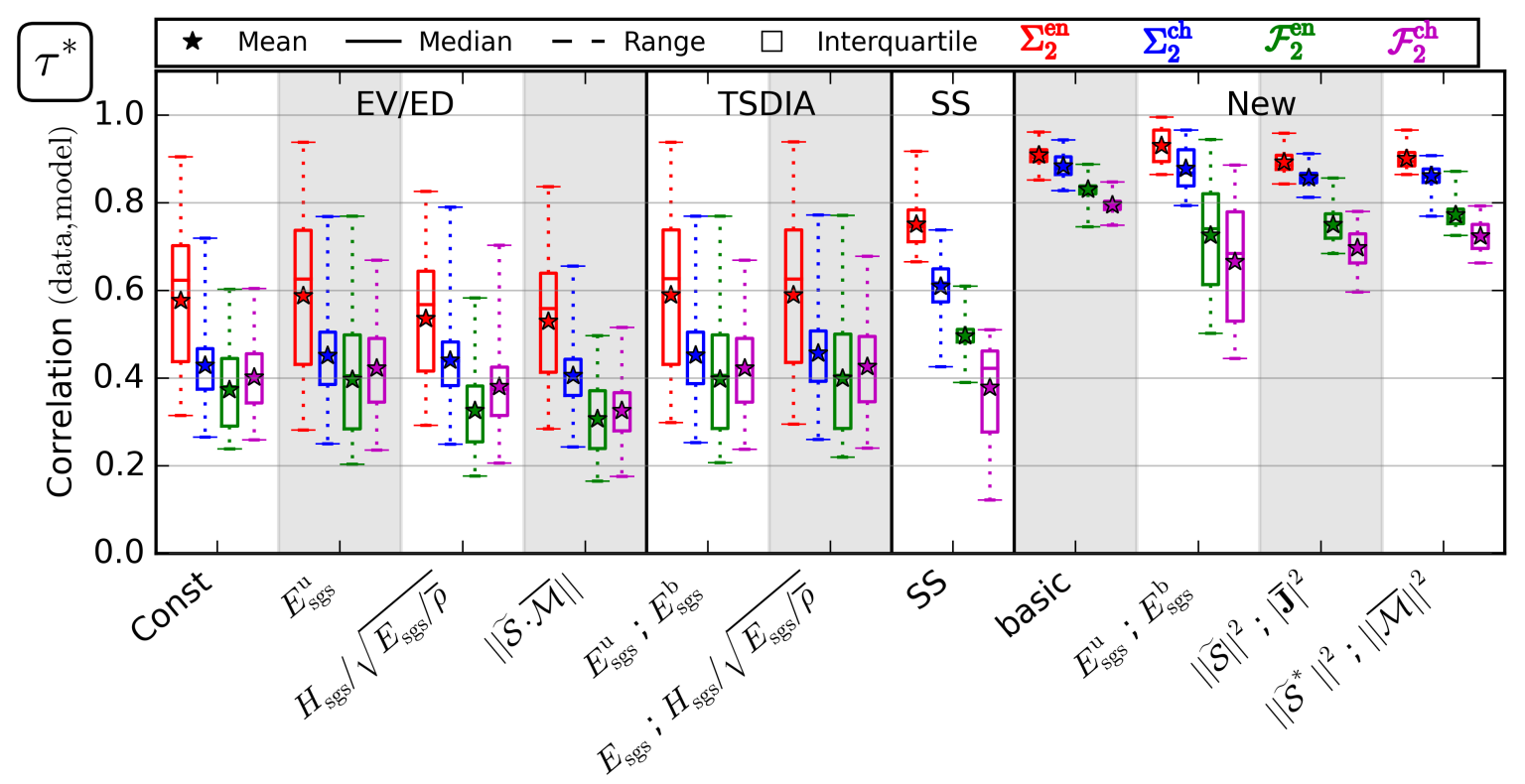

Figure 4.15: Correlation between data and model of the contributions of the term $\tau^{*}$ to the four diagnostic fields $\Sigma_{2}^{\text {en }}$ (red), $\Sigma_{2}^{\text {ch }}$ (blue), $\mathcal{F}_{2}^{\text {en }}$ (green) and $\mathcal{F}_{2}^{\text {ch }}$ (magenta). The models are grouped by families (EV,TSDIA, SS, and new) and labelled within the family by the characteristic modulator(s). For two coefficient models the two characteristic modulators are separated by a semicolon.

the EV family.

We show the flux distributions of the four diagnostic fields for the data and the models in fig. 4.16 for completeness. However, the distributions present no surprises. The TSDIA model performs poorly in the inverse cascade branch of the $\Sigma_{2}^{\mathrm{en}}$ distribution due to the suppression of the magnetic term and the inability of the kinetic one to represent two branches simultaneously. For the energy diagnostics in the sub- and trans-sonic simulations the TSDIA model is significantly closer to the data than in the respective kinetic comparison. This reaffirms its place within the EV family. The scale similarity and new models are again relatively close to the data both in the bulks and tails of their distributions.

We proceed with the structural comparison in fig. 4.17. The error angle is now affected by the values of the model coefficients, specifically their ratio regulates the relative importance of the orientations of the kinetic and magnetic components. In practice this effect is rather weak and we can afford to combine the error angles obtained from the four diagnostic fits in a single plot, fig. 4.17a. The orientation of all models relative to the data is consistent with the orientation of their kinetic and magnetic components. In the case of the TSDIA model the kinetic and magnetic error angles combine favourably producing a smaller error angle than in either of the kinetic or magnetic cases. However, the alignment is still very weak $-11.7 \%$ of the dataset is contained within $30^{\circ}$ of the data vector. For the scale similarity and new models this effect is altogether absent and the error angles lie between the respective kinetic and 


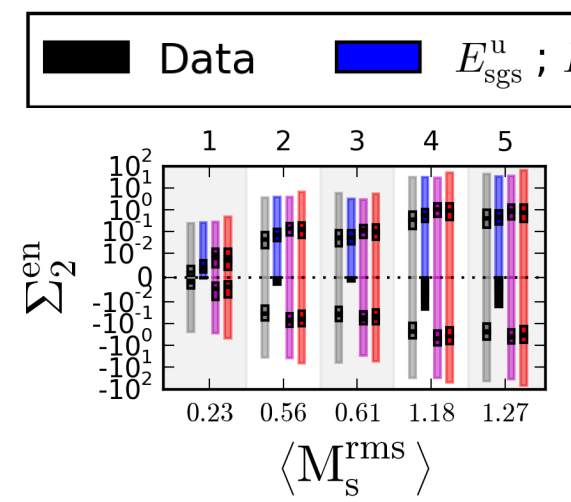

(a)

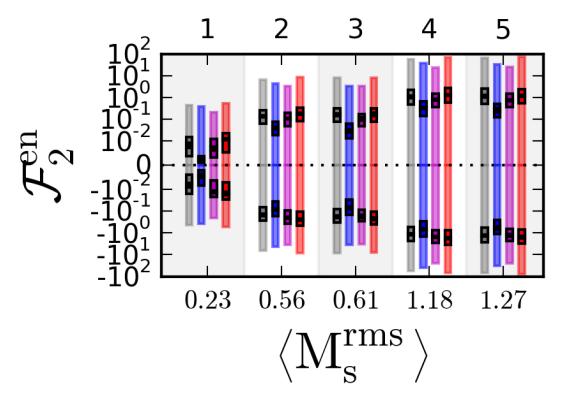

(c)

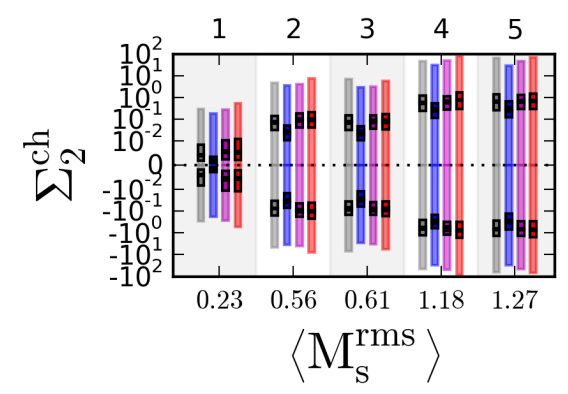

(e)

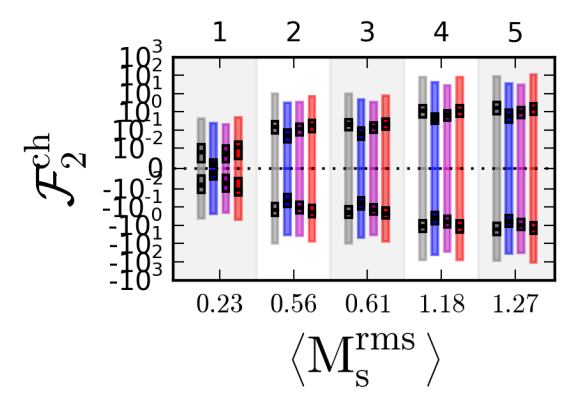

$(\mathrm{g})$

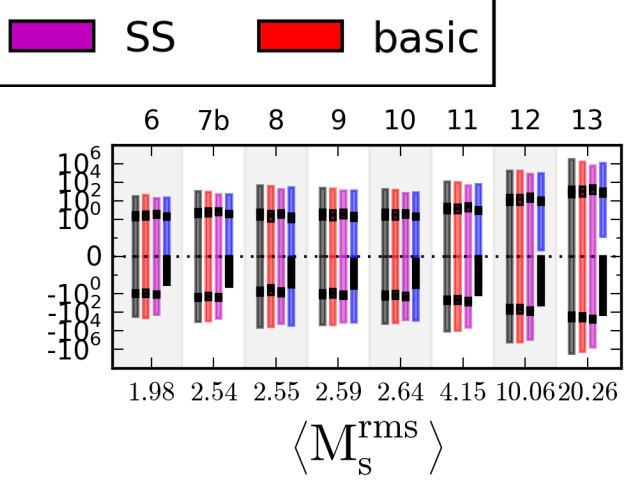

(b)

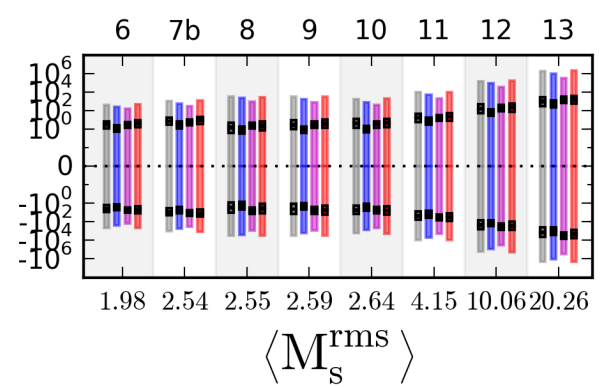

(d)

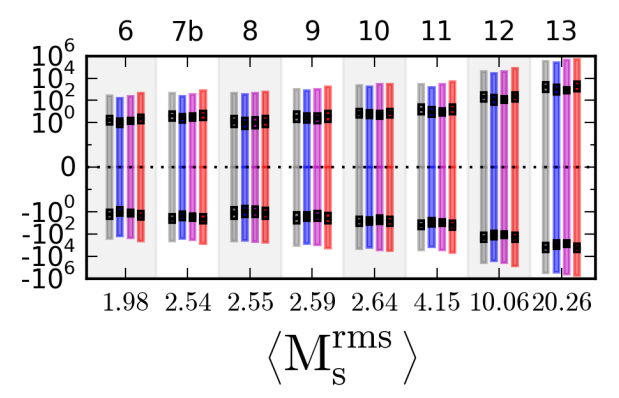

(f)

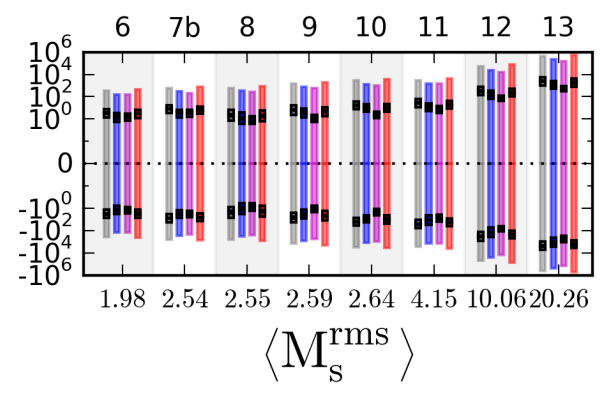

(h)

Figure 4.16: Summary of the flux distribution for the models of $\tau^{*}$ and the data: left column - sub- and trans-sonic simulations, right column - super-sonic simulations. Each row shows the contributions to one of the four diagnostic fields. The solid black rectangle represents the time variation of the median of each of the two branches of the distribution (positive down-scale transfer, negative - up-scale transfer). The empty black rectangle surrounding it shows the maximum (temporal) extent of the respective interquartile. The coloured bar shows the maximum (temporal) extent of the entire distribution. 


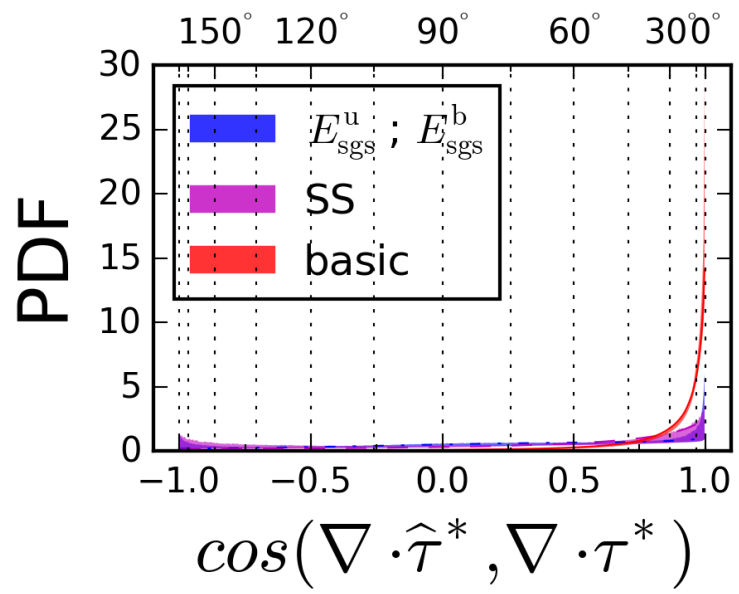

(a)

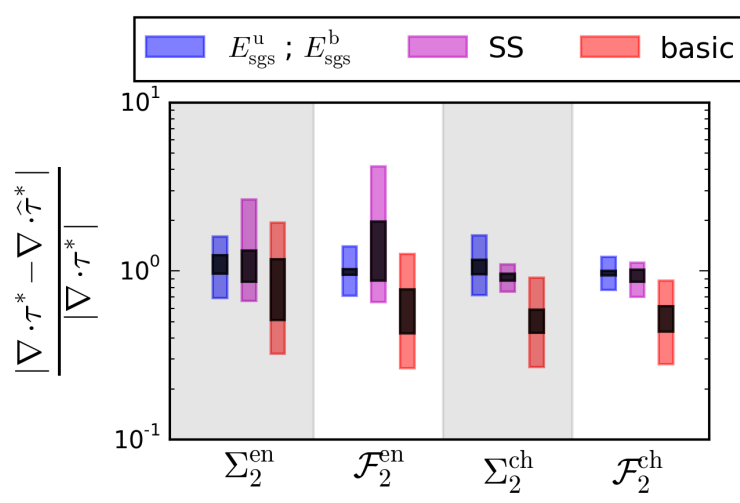

(b)

Figure 4.17: Geometry of the error vector of $\nabla \cdot\left(\tau^{*}\right)$ for the TSDIA $E_{\mathrm{sgs}}^{\mathrm{u}} ; E_{\mathrm{sgs}}^{\mathrm{b}}$ (blue), the SS (purple) and the basic new (red) models. Left panel: alignment angles between data and model with coefficients fitted from each of the diagnostic fields. The shaded area represents the variation across the entire dataset and all four diagnostic fits. The line represents the respective median. Right panel: size of the error vector normalised by the size of the data vector with coefficients fitted to each of the diagnostic fields. The black rectangle represents the variation across the entire dataset of the median of the distribution from each snapshot. The coloured rectangle represents the maximum extent across the entire dataset of the respective interquartile.

magnetic ones. In particular, for the SS model $24.6 \%$, and for the new model $61.7 \%$ of the dataset lies within $30^{\circ}$ of the data vector. It is expected that with the addition of the missing commutator term in the kinetic component the alignment may be raised to the levels of the magnetic case.

Similarly to all other considered quantities, the features of the relative error-vector size distributions are an agglomerate of the features of the kinetic and magnetic components (see fig. 4.17b). The new and scale-similarity models produce broader distributions with larger median with the energy and cross-helicity fits. The TSDIA model in contrast displays tighter distributions centred around unity for the total-flux terms and broader distributions skewed to larger values for the cascade terms. Concentrating on the new model alone, notably although the largest correlation is obtained for $\Sigma_{2}^{\mathrm{en}}$, this diagnostic field also produces the largest relative error vector.

We conclude the discussion by considering the tensor level comparison. Recall that in contrast to the kinetic and magnetic ones, the Q-R portrait of the total deviatoric SGS stress shows significant representation of both sheet and tube topologies. Figure 4.18 shows that all models can exhibit the same qualitative behaviour. However, as before the TSDIA and the SS models fail to reach the extreme values of $Q$ and $R$ that the data requires. Globally, the data is split approximately equally between the tube and sheet topologies with a tendency for sheet topology domination in the sub- and trans-sonic regimes. Figure 4.19 shows that TSDIA model overestimates the proportion of tube 

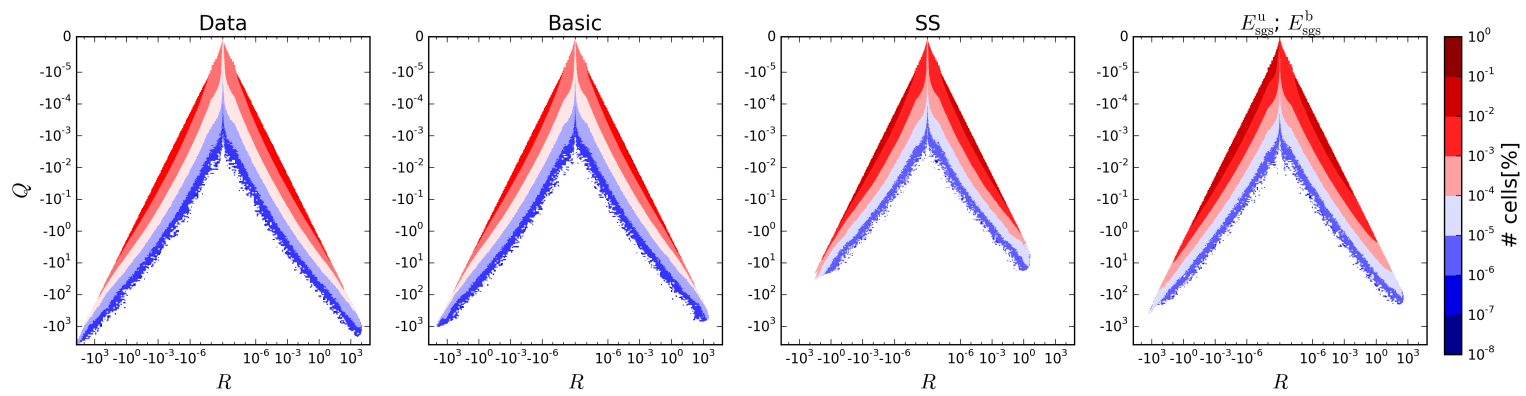

Figure 4.18: The Q-R portrait of $\tau^{*}$ for the data and selected models of a sample snapshot from simulation 9 with $\mathrm{M}_{\mathrm{s}, \mathrm{dr}}=2.9$ and $\beta_{\mathrm{p}, 0}=2.5$. The values are not normalised. The contours indicate the percentage of the cells with respect to the whole simulation domain.
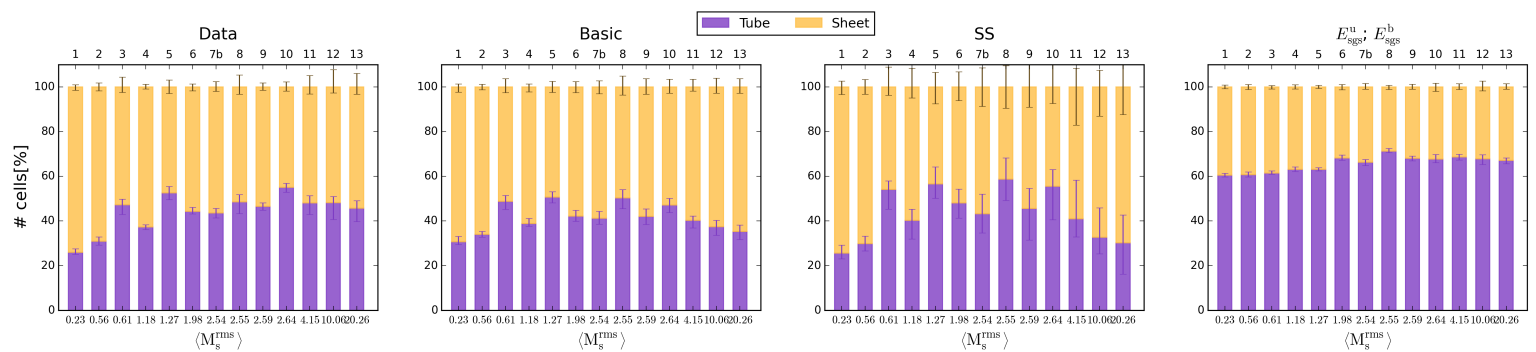

Figure 4.19: Summary of the distribution between the tube and sheet topologies for $\tau^{*}$ for all simulations. Error bars represent variations within the time interval between $2 T$ and $5 T$.

topologies and is somewhat less sensitive to the plasma parameters of the simulation than the data. The SS model is perhaps too sensitive as it shows much larger variations in time and with plasma parameters than the data. The new model follows the data's behaviour in time, however it underestimates the portion of tube topology in the largest sonic Mach number simulations by several per cent.

\subsection{Electromotive force: $\mathcal{E}$}

Finally, let us consider the EMF models. Five families of models can be identified ER, TSDIA, SS, new and mixed. The pure eddy-resistivity (ER) models (table 2.2) have the form $\mathcal{E} \propto \eta_{\mathrm{sgs}} \overline{\mathbf{J}}$. The dynamo-based TSDIA models are listed in table 2.3. As a simple case we examine an energy-based purely resistive option $(\alpha=\gamma=0$, $\left.\beta \propto\left(E_{\mathrm{sgs}} / \bar{\rho}\right)^{1 / 2}\right)$. This has the same vector form as an ER model, however the eddyresistivity here scales with the total SGS energy, instead of the magnetic SGS energy. This model shows most clearly the effect of the Smagorinsky and realizable energy closures on the EMF. Additionally, we compare the two full TSDIA models, using the true values for the SGS energy, residual-helicity and cross-helicity. This should gauge what is meant to be their optimal performance ${ }^{5}$. The scale-similarity model has its

\footnotetext{
${ }^{5}$ However, considering the difference between the different energy-based renormalization variants in
} the new SGS stress models, this expectation is not necessarily justified. 


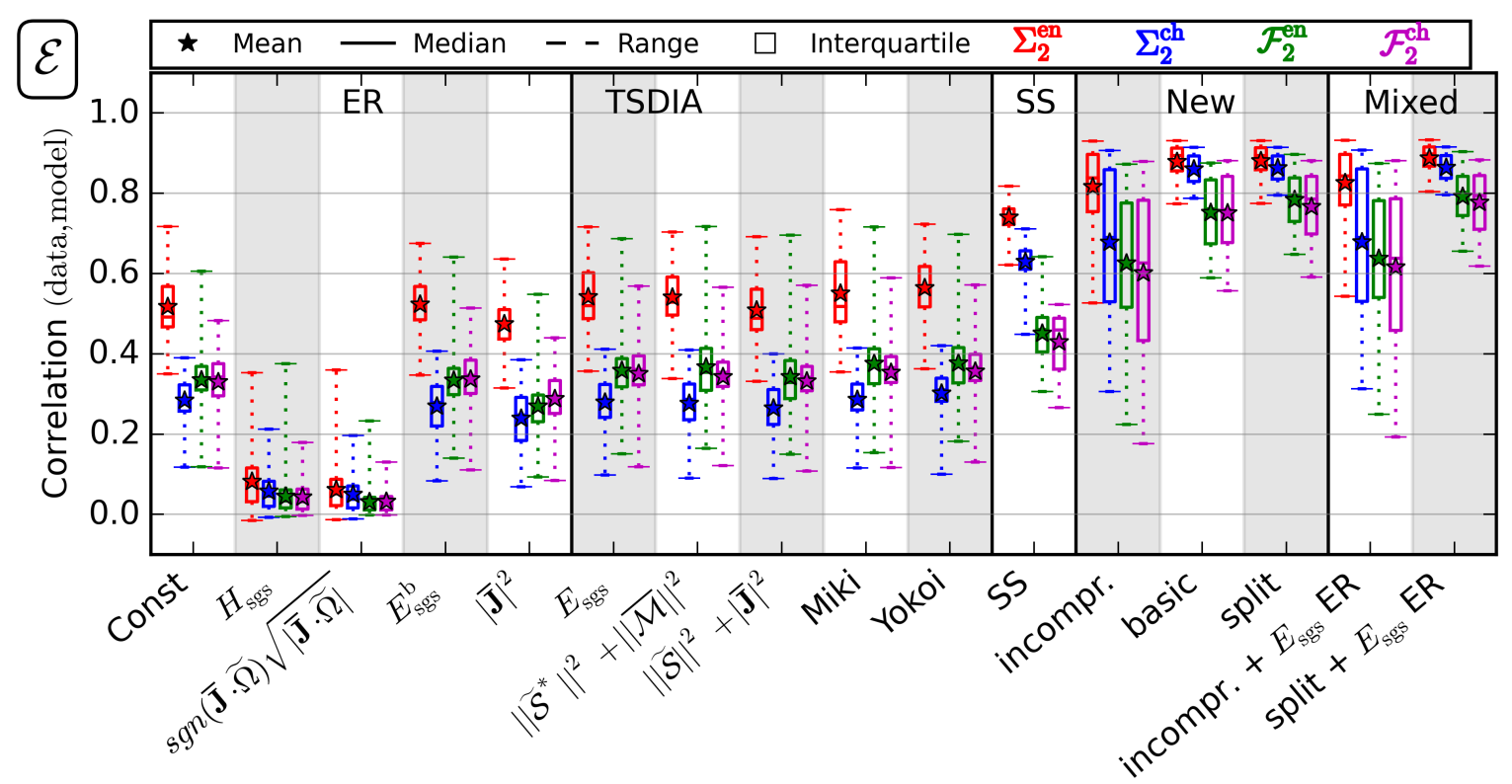

Figure 4.20: Correlation between data and model of the contributions of the term $\mathcal{E}$ to the four diagnostic fields $\Sigma_{2}^{\text {en }}$ (red), $\Sigma_{2}^{\text {ch }}$ (blue), $\mathcal{F}_{2}^{\text {en }}$ (green) and $\mathcal{F}_{2}^{\text {ch }}$ (magenta). The models are grouped by families (ER,TSDIA, SS, new and mixed) and labelled within the family by the characteristic modulator(s).

usual form. For the new model we consider three variants. The basic and split forms are described in table 2.1. Recall that they are simply a one- (basic) and two-coefficient (split) forms of the same model. As a reference we also fit just the incompressible term. The final set of mixed models consists of linear combinations of the true-energy-based purely resistive TSDIA model combined with the incompressible and basic forms of the new model. The correlations of all models are shown in fig. 4.20.

As in the case of the SGS stress, the best correlations for all models are with respect to the $\Sigma_{2}^{\mathrm{en}}$ diagnostic field. The difference here is that the cross-helicity cascade term $\Sigma_{2}^{\mathrm{ch}}$ does not necessarily have better correlations than the total flux terms.

Among the ER models we first note that the cross-helicity variants have negligible correlations with the data. Remarkably, the constant eddy-resistivity performs on the same level or better than the forms based on the SGS magnetic energy and the resolved current $^{6}$. This could be explained by the homogeneous and isotropic conditions, thanks to which the true SGS resistivity likely does not have large spatial variance. Overall, however, all models in this family have poor correlations which rarely exceeds 0.5.

The TSDIA models do not perform significantly better. The one-coefficient, energybased models yield similar results to the $E_{\mathrm{sgs}}^{\mathrm{b}}$ and $\overline{\mathbf{J}}$ forms of the ER models. Their primary advantage against the ER model is that they have somewhat improved correlations for the total flux terms. Notably, the three-coefficient models provide little improvement, hardly justifying the additional cost their implementation incurs. The reason for this is clarified by comparing the coefficients of the different terms. We

\footnotetext{
${ }^{6}$ i.e. the Smagorinsky magnetic energy closure
} 
observe (not shown) that the $\alpha$ and $\gamma$ the coefficients are not only much smaller than the $\beta$ coefficient, but also change their sign freely from one snapshot to the next. What is more, their mean and median values also change sign depending on the diagnostic field. This renders the Miki and Yokoi models effectively single coefficient models and explains their relation to the simple resistive model.

Nevertheless, note that in both families, reasonably high correlations can be reached in isolated snapshots, although the bulk of the correlations is quite poor. Examining the correlation spread across the different simulations, we note that in both families (excluding the cross-helicity based ER variants) there is a strong dependence between the correlations for the total flux diagnostics and the plasma parameters. In particular, the peak correlations are reached in the high Alfvénic Mach number simulations. This demonstrates the importance of testing models against a sufficiently large dataset - to ensure sufficient sampling of the parameter space.

The scale similarity model exhibits similar features to the ones of the SGS stress models. Note however that for most of the snapshots the total flux energy and cross-helicity fits yield similar correlations. Therefore, in contrast to the $\tau$-models, here one can reach the best accuracy of the model for both quantities with a single coefficient. With average correlations under 0.5 for the total flux terms however, this model is a moderate improvement over the functional models.

All new model variants also show better performance than the functional models. In the pure incompressible form however there are large variations. For the full flux terms the mean correlations decrease to $\sim 0.6$. A comparison to the basic form, which includes the compressibility term, clarifies a part of the reason. The mean correlation is improved, especially for the $\Sigma_{2}^{\text {ch }}$ field. The variations are also reduced significantly. We still however observe that the cascade terms yield significantly better correlations than the total flux ones. This is because the Favre-filtering - differentiation commutator is still missing. For the EMF it is given by

$$
(\widehat{\nabla \times \mathcal{E}}-\nabla \times \widehat{\mathcal{E}})_{i}=2 a \epsilon_{i j k} \epsilon_{k l m} \tilde{u}_{l, p} \bar{B}_{m} y_{, j p}
$$

Note that it has form similar to the compressible extension: $-2 a \nabla \times\left(\epsilon_{k l m} \tilde{u}_{l, p} \bar{B}_{m} y_{, p}\right)$ and the opposite sign. In fact they combine to give

$$
(\widehat{\nabla \times \mathcal{E}})_{i}=2 a \epsilon_{i j k} \epsilon_{k l m}\left(\left(\tilde{u}_{l} \bar{B}_{m}\right)_{j}-\left(\tilde{u}_{l, p} \bar{B}_{m}\right)_{j} y_{, p}\right)
$$

Thus we expect that some of the commutation error can be compensated for by adjusting independently the coefficient of the compressible extension. In fact the difference between the correlations of the basic and the split models is not very significant and could also be accounted for only by the additional degree-of-freedom of the latter. The total flux terms are also not significantly improved for the split model.

Consider, however, the coefficients of the basic and split models. As it can be seen in fig. 4.21, the overall coefficient of the basic model varies between 0.4 and 1.6. The two coefficients of the split model have similar ranges. However, the bulk of the variation is carried by the compressibility extension. Its median coefficient for the full flux fits 


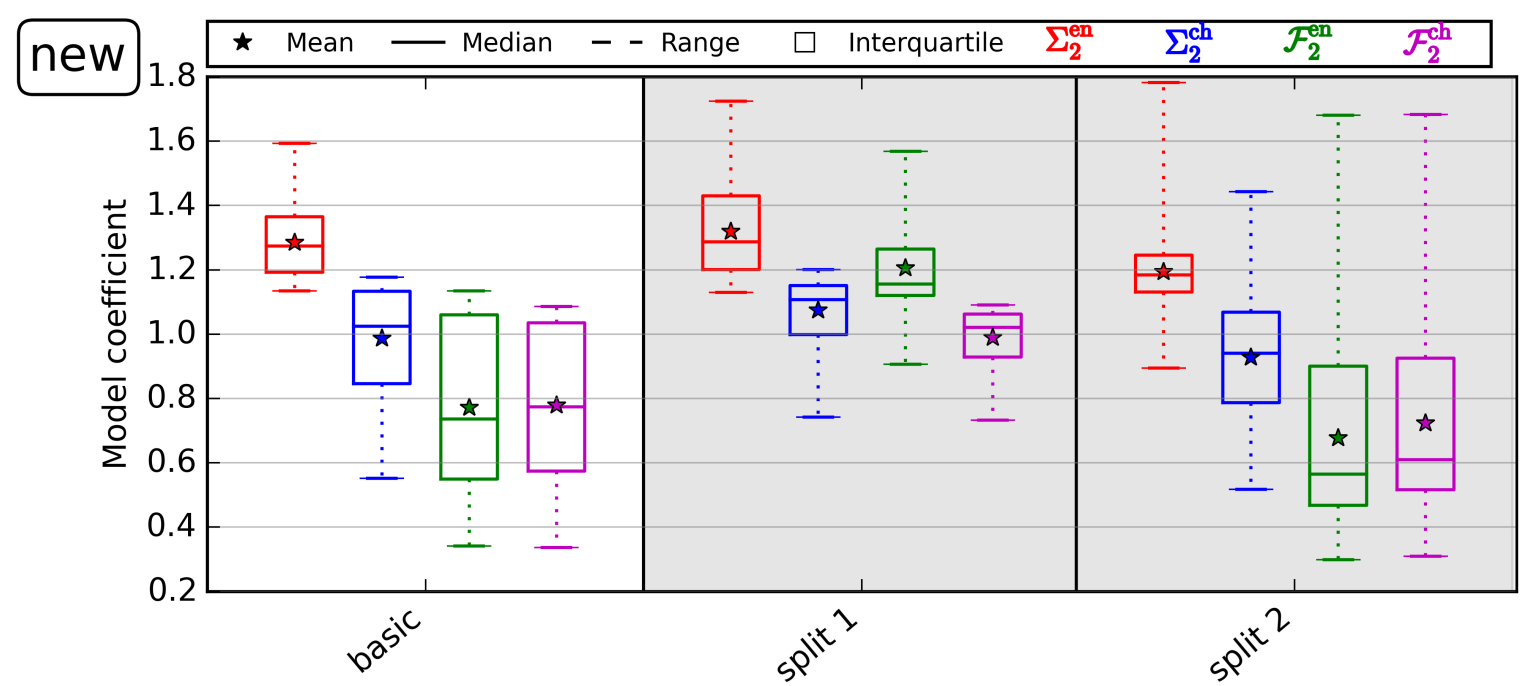

Figure 4.21: Summary of coefficient values obtained by fitting the contributions of the EMF to the four diagnostic fields $\Sigma_{2}^{\text {en }}$ (red), $\Sigma_{2}^{\text {ch }}$ (blue), $\mathcal{F}_{2}^{\text {en }}$ (green) and $\mathcal{F}_{2}^{\text {ch }}$ (magenta) for the new models. The first panel shows the coefficient of the basic, 1-coefficient model. The second set of panels shows the coefficients of the incompressible (1) and compressible (2) terms in the split model. The statistical features are as in fig. 4.20 .

is $\sim 0.6$ and closer to unity for the cascade fits. This is explained by the partial capturing of the commutator effect. Accounting for this, the absolute variations in the coefficients are quite small. The two coefficients of the split model are sufficiently close to each other and to the coefficient of the basic model. Therefore, we maintain that these results validate the amplitude of the EMF model and precipitate any need for renormalization.

Finally, the mixed models behave in just the same manner as they did for the SGS kinetic and magnetic stress. The coefficient of the eddy-resistivity component is reduced to negligence by the fit and the models are rendered equivalent to the corresponding new model variants.

We continue with the more detailed comparison of a selection of models. We consider the constant eddy resistivity model, the Yokoi model, the scale-similarity and the basic new model as they exhibit the highest correlations in their respective families.

Beginning with the flux distributions, fig. 4.22, just as in the case of the kinetic SGS stress, the ER model cannot and the TSDIA model struggles to represent the inverse energy cascade, i.e. the dynamo action, especially for the cascade diagnostics. The transfer from large to small scales on the other hand is represented generally well by all models for most simulations. Surprisingly, here the functional models also seem to perform better in the supersonic regime. The scale similarity and new models exhibit similar behaviour with respect to the sonic Mach number. However, they follow closely the core of the data distribution in both positive and negative branches for all diagnostic fields. The overall range of the data is more poorly represented, with all 
models consistently falling short in most simulations.

Structurally, since the EMF is a (pseudo-)vector itself no tensor analysis is necessary. Instead, at the vector level, we can examine two relevant error vectors - the ones related to $\mathcal{E}$ and $\nabla \times \mathcal{E}$. As the values of the model coefficients affect the error-angle as well as the error-vector size for multi-coefficient models, we take them from the cascade fits for the $\mathcal{E}$ error vector and from the total full flux fits for the $\nabla \times \mathcal{E}$ error vector. For consistency we do the same for the one-coefficient models. This maintains the same sample space across all models.

The results for the error angles are shown in fig. 4.23. The $\mathcal{E}$ alignment is much better than the $\nabla \times \mathcal{E}$ one. We note that the new model demonstrates the strongest alignment with $68.6 \%$ of the dataset contained within $30^{\circ}$ of the $\mathcal{E}$. This number reduces to $56.8 \%$ for $\nabla \times \mathcal{E}$. Naturally, the reduction is a consequence of the missing commutator effect and affects all models. For the scale-similarity model the effect is quite strong $-42.4 \%$ for the $\mathcal{E}$ error angle and $22.1 \%$ for the $\nabla \times \mathcal{E}$ error angle. In other words the scale-similarity model shows a non-negligible alignment to the pure EMF vector. This suggests that similar level of alignment holds for the eigenvectors of the SS models of the SGS stress as well but is obscured in the error-angles of SGS force due to the missing differentiation commutator. The TSDIA model is largely insensitive to the orientation of the EMF. This is expected since the TSDIA model is effectively oriented along the resolved current and, cf. section 3.2, the resolved current and the EMF are not mutually aligned.

The relative error-vector size are again of order unity as seen in fig. 4.24. There is no robust differentiation between the $\mathcal{E}$ and $\nabla \times \mathcal{E}$ fits across all models. On the one hand, the TSDIA and ER models exhibit larger variation for $\nabla \times \mathcal{E}$ than $\mathcal{E}$, while the SS model shows the reverse. On the other, the ER and new models have similar median values and variations in both cases. Combining these observations with the flux correlation we conclude, that also in the case of the EMF the error angle is the key dynamical quantity linked to the cross-scale transfer, while the amplitude of the model vector plays a secondary role.

\subsection{Summary}

We finish this chapter by summarizing the results we have obtained for all SGS terms. The presented analysis leads to the conclusion that the diffusion effects exhibited by the $\mathcal{F}_{2}^{\text {en }}$ and $\mathcal{F}_{2}^{\text {ch }}$ fields can change the view on the models' performance significantly and should be considered in any comprehensive model test. Moreover, even in the case of homogeneous and isotropic turbulence, as represented by the examined dataset, the local orientation of the SGS terms plays a key role in their dynamic effect. Therefore detailed geometrical diagnostics are necessary also when performing functional tests. In light of this we used the following quantities to gauge the different models' performance against the data and each other.

1. Direct correlation to the data.

2. Overall flux correlations for each of the diagnostic fields $\Sigma_{2}^{\mathrm{en}}, \Sigma_{2}^{\mathrm{ch}}, \mathcal{F}_{2}^{\mathrm{en}}$ and $\mathcal{F}_{2}^{\mathrm{ch}}$. 


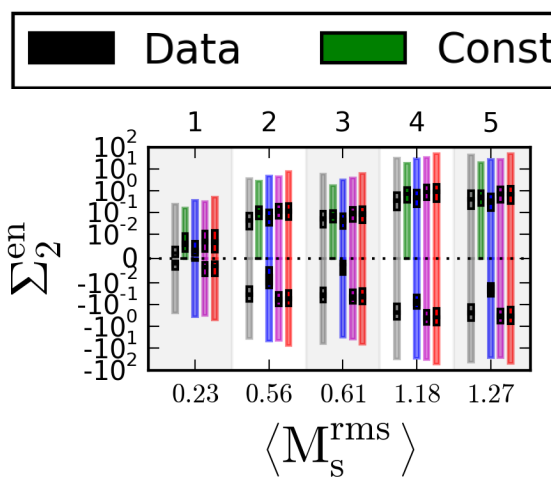

(a)

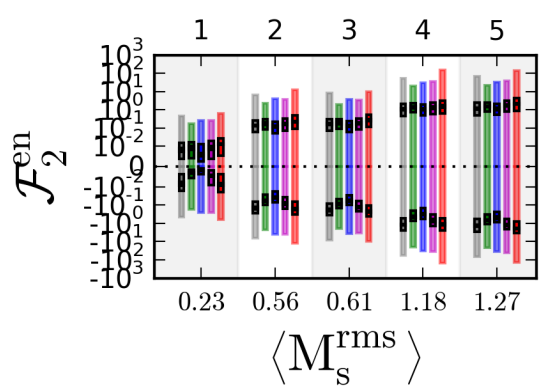

(c)

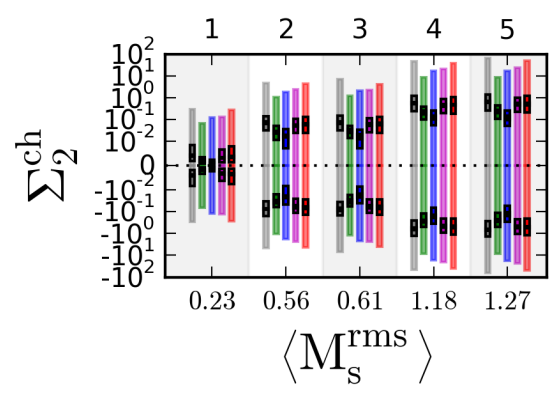

(e)

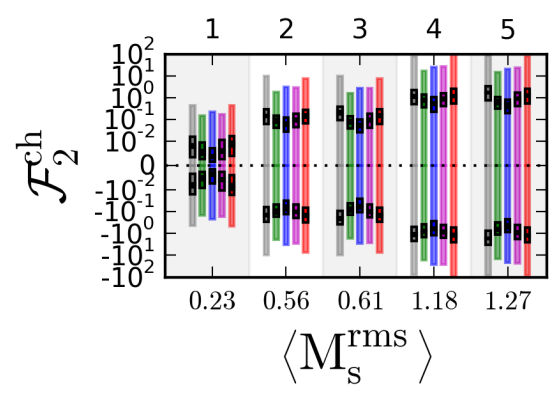

$(\mathrm{g})$

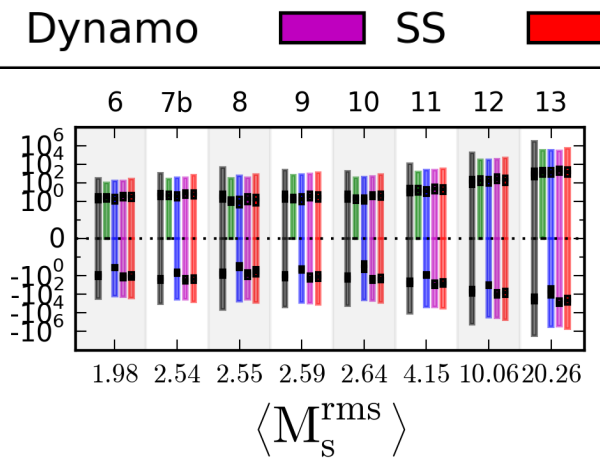

(b)

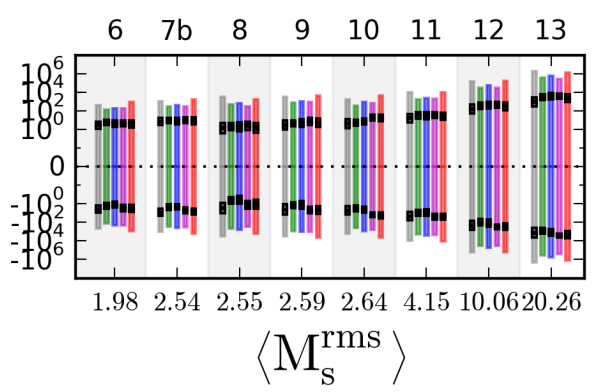

(d)

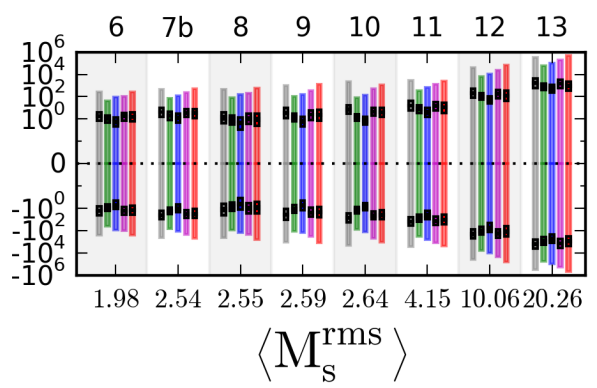

(f)

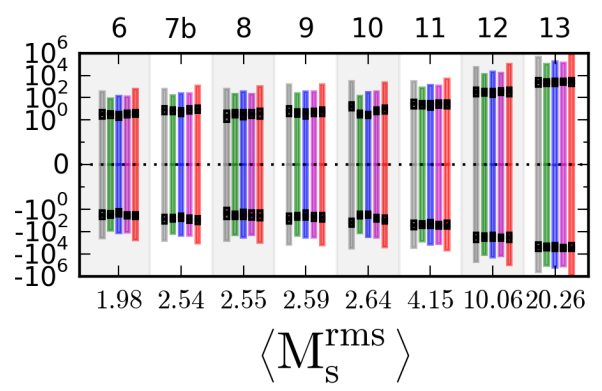

(h)

Figure 4.22: Summary of the flux distribution for the models of $\mathcal{E}$ and the data: left column - sub- and trans-sonic simulations, right column - super-sonic simulations. Each row shows the contributions to one of the four diagnostic fields. The solid black rectangle represents the time variation of the median of each of the two branches of the distribution (positive down-scale transfer, negative - up-scale transfer). The empty black rectangle surrounding it shows the maximum (temporal) extent of the respective interquartile. The coloured bar shows the maximum (temporal) extent of the entire distribution. 


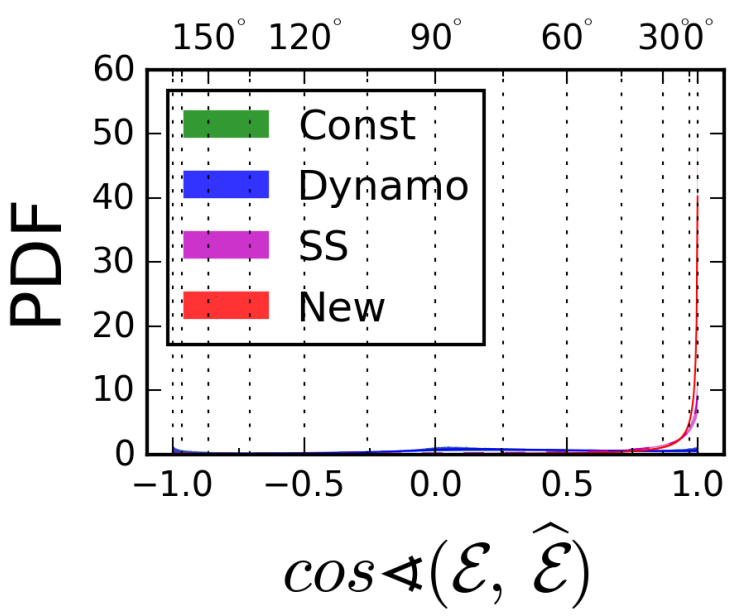

(a)

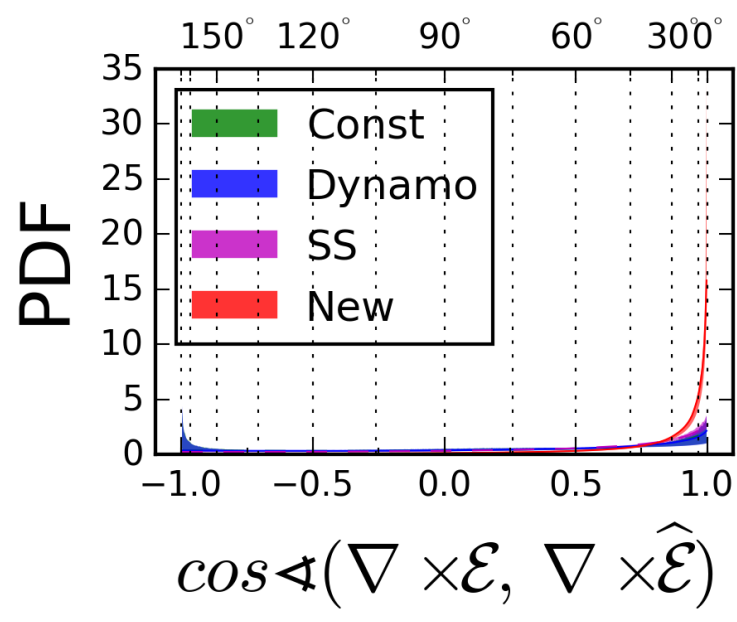

(b)

Figure 4.23: Alignment angles between data and model for: $\mathcal{E}$, with coefficients fitted from cascade diagnostics (left panel), $\nabla \times \mathcal{E}$, with coefficients fitted from total flux diagnostics (right panel).

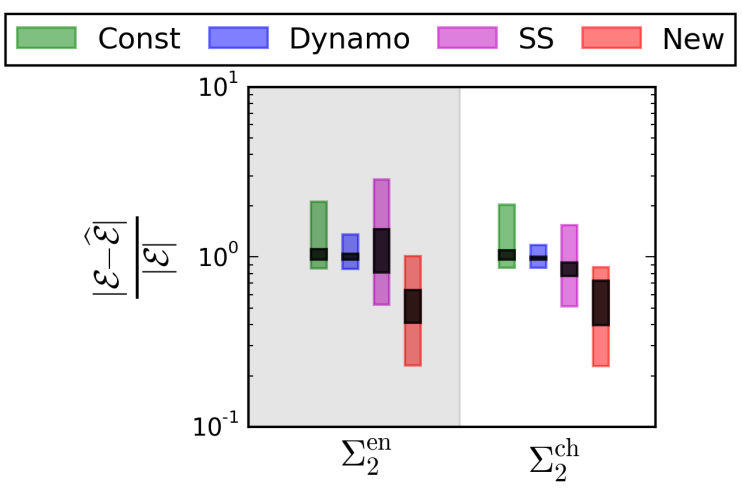

(a)

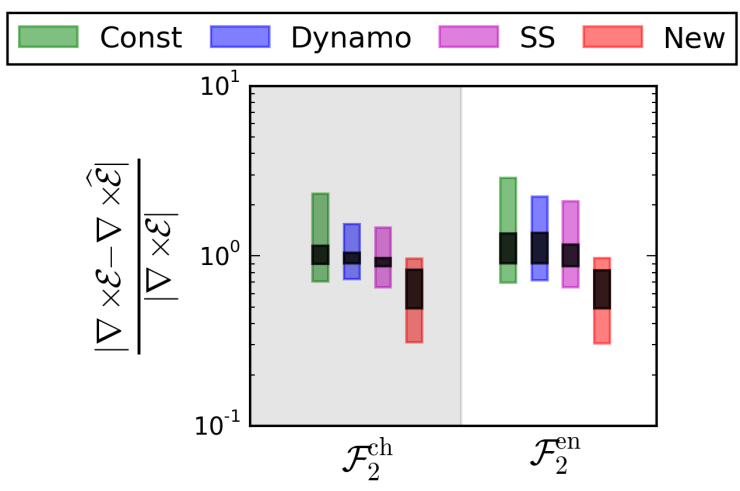

(b)

Figure 4.24: Size of the error vector normalised to the size of the data vector for: $\mathcal{E}$, with coefficients fitted from cascade diagnostics (left panel), $\nabla \times \mathcal{E}$, with coefficients fitted from total flux diagnostics (right panel). 
3. Spatial flux distributions for each of the diagnostic fields.

4. Error vector geometry - error angle and relative error vector size.

5. Quadratic tensor invariants and distribution across topological regions.

They present complimentary views on the models' performance. The direct correlation is precise but of limited use for the SGS tensor and vector terms. It provides an instantaneous and local view. The SGS flux correlations are the most sensitive diagnostic and are able to separate the different models most clearly. However, they carry no information on the cause for a particular model's performance. The remaining vector and tensor diagnostic are global (in a spatial sense) and thus less sensitive to quantitative differences between the models. The error angle and topological region distribution correspond well with the flux correlations and thus explain these results. While this connection merits deeper investigation, it is clearly based on the fact that flux correlations are related to the eigenvalues and eigenvectors of the SGS terms just as the error-angle and topological region distributions are. The relative size of the error vector and the flux distributions on the other hand, have a non-trivial non-monotonic relationship to the flux correlations. Thus they order the models' performance differently in some cases. Due to the difficulties in interpretation of this order however, we follow the model order given by the local flux correlations. In both cases however, the new model outperforms its alternatives by a significant margin.

Renormalizing the SGS stress is not beneficial a priori. The amplitude of the basic models correlates well with the local turbulence strength as measured by the respective SGS energy. Additionally, for the majority of snapshots the flux distributions of the basic models are contained within the boundaries of the data distributions. This suggest that the model should not cause spurious numerical instabilities. If renormalization is required a posteriori, then using a Smagorinsky or a realizable energy closure is preferable to using the true SGS energy.

For all models the closures of the differentiated SGS terms (except the $\tau^{\mathrm{b}}$ ones) are missing an important component due to the Favre-filtering - differentiation commutator. For the new model it is explicitly calculated and it turns out to be of leading order, $O(a)$. The comparison to diagnostics unaffected by this error suggests that the inclusion of this term would improve the model's performance. However, additional a priori testing is required to validate this. In any case, since all evolution equations involve differentiation of the SGS terms, the inclusion of some commutator correction is essential to a posteriori application.

The scale-similarity models seem to neglect a major ingredient in addition to this commutator term, since its performance with respect to the cross-helicity diagnostics is consistently worse than with respect to the energy ones.

The TSDIA and EV/ED/ER models show unsatisfactory performance with respect to the local flux correlations due to their geometrical structure. Their global performance varies significantly across diagnostic terms and within the dataset. 



\section{Conclusions and Outlook}

Turbulent flows are ubiquitous in nature and especially so on astrophysical scales, where microscopic viscosity is often negligible. Examples can be found on all scales - from the intra-cluster medium, through galactic molecular clouds, all the way to stellar and planetary atmospheres. Compressibility effects are often a major dynamical component which shapes the evolution of these systems. Moreover, many of these phenomena are strongly influenced by the presence of external or self-generated magnetic fields. Thus the simplest theory which can be used to describe them in any degree of completeness is compressible magnetohydrodynamics.

Numerical simulations form an essential part of turbulent MHD research. However, the extreme parameter regimes found in astrophysics and the finite computational resources often prohibit the direct simulation of all dynamically relevant scales. Only a sub-range of scales can be simulated self-consistently, while the remaining scales are included via an explicit or implicit model. In the LES approach such a model is attained by describing the finite resolution effects with a low-pass filtering operator. This operator is applied to the MHD equations and the terms associated with the finite resolution effects are identified. Due to the origins of this approach in grid-based simulations, they are dubbed sub-grid scale terms. The momentum and induction equations give rise to a SGS stress and an EMF terms, respectively.

In this work we presented a new model for these terms based on a gradient expansion of the filtering operator developed by [103] for hydrodynamic applications. Specifically, we extended the approach to include SGS terms derived from a mass-weighted filtering operator, the latter being quite common in compressible MHD applications. For comparison, we provided an overview of the main alternative models which are currently in circulation. They can be divided into several families according to their basic assumptions. The scale similarity model is based on the idea that the structure of the unresolved turbulent scales is similar to the one of the resolved ones. Alternatively, in the eddy-viscosity/diffusivity/resistivity family the turbulent effects on the resolved scales are seen merely as a spatially-dependent modulation of the respective microphysical processes. Closely related is the TSDIA model which is inspired by analytical work based on statistical averaging of the evolution equations.

The comparison of the different models was performed in an a priori fashion. This consists of the explicit filtering of highly resolved simulation data and comparing the accurate SGS terms produced by that operation with the model predictions based only on the large-scale 'resolved' part of the data. We used a set of simulations from two different finite-volume grid-based codes, ENZO and FLASHv4, in order to reduce the systematic code-dependent bias. The simulated flow is that of statistically stationary homogeneous and isotropic, approximately isothermal turbulence under the effect of large-scale stochastic forcing, which acts only on the momentum equation. The initial 
plasma beta parameter range is $\beta_{\mathrm{p}, 0} \in[0.05,450]$. In the stationary regime, which spans approximately 3 eddy-turnover times, the RMS Mach number range is $[0.2,20]$ and the corresponding Alfvénic Mach numer range is [0.5,3.4]. The effective kinetic and magnetic Reynolds numbers are of the order of $10^{3}$.

The first task we pursued was to establish properties of the SGS terms, which come from the momentum, induction, energy and cross-helicity equations. They are detailed in chapter 3. There we emphasized several key points with respect to the hypothesis used for model building. Firstly, we found that the local balance hypothesis used to derive the Smagorinsky energies is not supported by the data. On the contrary, the dominant part of both energy and cross-helicity transfer across the filter scale is due to transport terms. The SGS transfer between the kinetic and magnetic budgets is the dominant part in the evolution of the respective SGS energies. Secondly, the electromotive force cannot be faithfully modelled by a simple resolved vector, as suggested in both EV and TSDIA models, due to their poor mutual alignment. Thirdly, the SGS pressure which is often ignored or treated implicitly needs to be given full consideration, as it contributes a significant part to the momentum flux budget throughout the whole range of parameters including the subsonic regime. Throughout the examined dataset the amplitude of the SGS stress is driven by the SGS pressure, while the deviatoric component dictates its orientation. Additionally, we found that the spatial distribution of the SGS kinetic and magnetic stress terms between tube and sheet topologies is a very robust signature of the turbulent flow.

The second task was the direct comparison of the models with data and among themselves. We selected a range of structural and functional, local and global diagnostics in order to provide a comprehensive view of the models' performance. Functionally, we considered the contribution of the SGS stress and the EMF to the energy and crosshelicity transfer across the filter scale. Structurally, for scalar quantities we computed the correlation between data and model; for vector quantities we analysed the error angle and error vector; for tensor quantities we analysed the distributions of the quadratic invariants and the distribution between sheet and tube topologies. The main result of this work is that the proposed model outperformed all alternatives in all conducted comparisons. We attributed this to a better local adherence to the geometrical structure of the SGS terms. The main disadvantage of the eddy-viscosity models is that they only accommodate one direction of energy transfer - from large to small scales. Furthermore, the functional models in general are designed to model, what turned out to be in the examined data set, a sub-dominant part of the energy transfer. Thus their overall performance with respect alternative diagnostics suffered. The scale-similarity model performed similarly to the proposed model with regards to structural diagnostics, however functionally it neglects the cross-helicity dynamics and thus struggled to represent as faithfully the energy and cross-helicity total flux terms. It must be remarked however that all models suffered from a commutation error between the mass-weighted filtering operator and differentiation which affected their performance with respect to the total flux terms. Overall, the a priori analysis identified the proposed models as the preferred candidate for further investigation and applications.

The next steps of model verification include an expansion of the paramater space to include non-isotropic flows, decaying turbulence, self-gravity and thermal effects. 
Additionally, the influence of the filter kernel shape needs to be investigated along with the filter scale. The latter requires higher resolution data with sufficiently broad inertial range to accommodate several sufficiently distinct values for the filter scale. From the analytical point view, the relationship between the error vector and the flux correlations needs to be clarified in order to better understand the reasons for the performance of the different models, i.e. to what extent the different diagnostics support and/or explain one another. Additionally, the effects of the Favre-filter - differentiation commutator need to be verified.

In parallel, a posteriori analysis can already be performed on the proposed model. The results can then be compared to a corresponding DNS or experimental dataset. The current results provide strong motivation to begin the testing with static constant coefficients. The a posteriori analysis will require the selection of a new set of diagnostics, but it will also explore a new facet of the model. For instance, a posteriori tests provide a direct access to temporally non-local model features. Ultimately, our goal is to use the model in realistic simulations directed at addressing some of the outstanding astrophysical problems of the day. Hopefully, this will make a small step in the direction of understanding the dynamics of compressible MHD turbulence in astrophysics. 



\section{Bibliography}

[1] Agullo, O., Müller, W.-C., Knaepen, B. \& Carati, D. (2001) Large eddy simulation of decaying magnetohydrodynamic turbulence with dynamic subgridmodeling. Phys. Plasmas, 8(7), 3502.

[2] Aluie, H. (2011) Compressible Turbulence: The Cascade and its Locality. Phys. Rev. Lett., 106(17), 174502.

[3] Aluie, H. (2013) Scale decomposition in compressible turbulence. Physica D Nonlinear Phenomena, 247, 54-65.

[4] Balarac, G., Kosovichev, A. G., Brugière, O., Wray, A. A. \& ManSOUR, N. N. (2010) Modeling of the subgrid-scale term of the filtered magnetic field transport equation. In Summer Progr. 2010 (Center Turbul. Res. Stanford Univ.

[5] Balarac, G., Le Sommer, J., Meunier, X. \& Vollant, A. (2013) A dynamic regularized gradient model of the subgrid-scale scalar flux for large eddy simulations. Phys. Fluids, 25(7), 075107.

[6] Balarac, G., Pitsch, H. \& Raman, V. (2008) Development of a dynamic model for the subfilter scalar variance using the concept of optimal estimators. Phys. Fluids, 20(3), 10.

[7] Balbus, S. A. \& Hawley, J. F. (1998) Instability, turbulence, and enhanced transport in accretion disks. Rev. Mod. Phys., 70(1), 1-53.

[8] Banerjee, S. \& Galtier, S. (2013) Exact relation with two-point correlation functions and phenomenological approach for compressible magnetohydrodynamic turbulence. Phys. Rev. E - Stat. Nonlinear, Soft Matter Phys., 87(1), $1-8$.

[9] Bardina, J., Ferziger, J. H. \& Reynolds, W. C. (1983) Improved turbulence models based on large eddy simulation of homogeneous, incompressible turbulent flows. Tech. Rep. May 1983, Stanford University.

[10] Bin, L. \& Songping, W. (2007) A Nonlinear Sub-grid Scale Model for Compressible Turbulent Flow. Chinese J. Aeronaut., 20, 495-500.

[11] Biskamp, D. (2003) Magnetohydrodynamic Turbulence. CUP.

[12] Bruno, R. \& Carbone, V. (2013) The Solar Wind as a Turbulence Laboratory. Living Rev. Sol. Phys., 10(1), 1-208. 
[13] Budaev, V. P., Savin, S. P. \& Zelenyi, L. M. (2011) Investigation of intermittency and generalized self-similarity of turbulent boundary layers in laboratory and magnetospheric plasmas: towards a quantitative definition of plasma transport features. Uspekhi Fiz. Nauk, 181(9), 905.

[14] Carati, D., Winckelmans, G. S. \& Jeanmart, H. (2001) On the modelling of the subgrid-scale and filtered-scale stress tensors in large-eddy simulation. $J$. Fluid Mech., 441, 119-138.

[15] Chernyshov, A. A., Karelsky, K. V. \& Petrosyan, A. S. (2006) Largeeddy simulation of magnetohydrodynamic turbulence in compressible fluid. Phys. Plasmas, 13(3), 032304.

[16] Chernyshov, A. A., Karelsky, K. V. \& Petrosyan, A. S. (2007) Development of large eddy simulation for modeling of decaying compressible magnetohydrodynamic turbulence. Phys. Fluids, 19(5), 055106.

[17] Chernyshov, A. A., Karelsky, K. V. \& Petrosyan, A. S. (2010) Forced turbulence in large-eddy simulation of compressible magnetohydrodynamic turbulence. Phys. Plasmas, 17(10), 102307.

[18] Chernyshov, A. A., Karelsky, K. V. \& Petrosyan, A. S. (2014) Subgridscale modeling for the study of compressible magnetohydrodynamic turbulence in space plasmas. Physics-Uspekhi, 57(5), 421-452.

[19] Cho, J. (2014) Origin of Magnetic Field in the Intracluster Medium: Primordial or Astrophysical? Astrophys. J., 797(2), 133.

[20] Chong, M. S., Perry, A. E. \& Cantwell, B. J. (1990) A general classification of three-dimensional flow fields. Phys. Fluids A Fluid Dyn., 2(5), 765.

[21] Chong, M. S., Soria, J., Perry, A. E., Chacin, J., Cantwell, B. J. \& NA, Y. (1998) Turbulence structures of wall-bounded shear flows found using DNS data. J. Fluid Mech., 357, S0022112097008057.

[22] Clark, R. A., Ferziger, J. H. \& Reynolds, W. C. (1979) Evaluation of subgrid-scale models using an accurately simulated turbulent flow. J. Fluid Mech. $z<a, \mathbf{9 1}(01), 16$.

[23] Comte, P. \& Leisieur, M. (1998) Large-eddy simulations of compressible turbulent flows. In Advances in turbulence modeling, Lecture Series, von Karman Institute for Fluid Dynamics, pp. 1998-05.

[24] Dallas, V. \& Alexakis, A. (2013) Structures and dynamics of small scales in decaying magnetohydrodynamic turbulence. Phys. Fluids, 25(10), 105106.

[25] Davidson, P. A. (2004) Turbulence: an introduction for scientists and engineers. Oxford University Press. 
[26] Dedner, A., Kemm, F., Kröner, D., Munz, C.-D., Schnitzer, T. \& WeSEnBerG, M. (2002) Hyperbolic Divergence Cleaning for the MHD Equations. J. Comput. Phys., 175(2), 645-673.

[27] Djorgovski, S. G., Castro, S., Stern, D. \& Mahabal, A. A. (2001) On the Threshold of the Reionization Epoch. Astrophys. J., 560(1), L5-L8.

[28] Dobler, W., Haugen, N. E. L., Yousef, T. A. \& Brandenburg, A. (2003) Bottleneck effect in three-dimensional turbulence simulations. Phys. Rev. E, 68(2), 026304 .

[29] Domaradzki, J. A. \& SAiki, E. M. (1997) A subgrid-scale model based on the estimation of unresolved scales of turbulence. Phys. Fluids, 9(7), 2148.

[30] Erlebacher, G., Hussaini, M. Y., Speziale, C. G. \& Zang, T. A. (1992) Toward the large-eddy simulation of compressible turbulent flows. J. Fluid Mech., 238, 155-185.

[31] Fabre, Y. \& Balarac, G. (2011) Development of a new dynamic procedure for the Clark model of the subgrid-scale scalar flux using the concept of optimal estimator. Phys. Fluids, 23(11), 115103.

[32] Fan, X., Narayanan, V. K., Lupton, R. H., Strauss, M. A., Knapp, G. R., Becker, R. H., White, R. L., Pentericci, L. et Al. (2001) A Survey of z $>5.8$ Quasars in the Sloan Digital Sky Survey I: Discovery of Three New Quasars and the Spatial Density of Luminous Quasars at z $\sim$. Astron. J., $\mathbf{1 2 2}(6), 40$.

[33] Fan, X., Narayanan, V. K., Lupton, R. H., Strauss, M. A., Knapp, G. R., Becker, R. H., White, R. L., Pentericci, L. et Al. (2002) A Survey of z>5.8 Quasars in the Sloan Digital Sky Survey I: Discovery of Three New Quasars and the Spatial Density of Luminous Quasars at z $\sim$. Astron. J., $\mathbf{1 2 2}(6), 40$.

[34] Fan, X., Strauss, M. A., Becker, R. H., White, R. L., Gunn, J. E., Knapp, G. R., Richards, G. T., Schneider, D. P. et Al. (2006) Constraining the Evolution of the Ionizing Background and the Epoch of Reionization with z 6 Quasars. II. A Sample of 19 Quasars. Astron. J., 132(1), 117-136.

[35] Fan, X., Strauss, M. A., Schneider, D. P., Becker, R. H., White, R. L., Haiman, Z., Gregg, M., Pentericci, L. et Al. (2003) A Survey of z>5.7 Quasars in the Sloan Digital Sky Survey. II. Discovery of Three Additional Quasars at z 6. Astron. J., 125(4), 1649-1659.

[36] Favre, A. (1983) Turbulence: Space-time statistical properties and behavior in supersonic flows. Phys. Fluids, 26(10), 2851.

[37] Federrath, C. (2013) On the universality of supersonic turbulence. Mon. Not. R. Astron. Soc., 000(June), 14. 
[38] Federrath, C., Roman-Duval, J., Klessen, R. S., Schmidt, W. \& Mac Low, M.-M. (2010) Comparing the statistics of interstellar turbulence in simulations and observations. Astron. Astrophys., 512, A81.

[39] Fortov, V. E., Khrapak, A. G., Khrapak, S. A., Molotkov, V. I. \& Petrov, O. F. (2004) Dusty plasmas. Physics-Uspekhi, 47(5), 447-492.

[40] Frisch, U. (1996) Turbulence: the legacy of A. N. Kolmogorov. Reprint. edn., Cambridge Univ. Press, Cambridge [u.a.].

[41] Fryxell, B., Olson, K., Ricker, P., Timmes, F. X., Zingale, M., Lamb, D. Q., MacNeice, P., Rosner, R. et Al. (2000) FlASH: An Adaptive Mesh Hydrodynamics Code for Modeling Astrophysical Thermonuclear Flashes. Astrophys. J. Suppl. Ser., 131(1), 273-334.

[42] Galtier, S. \& Banerjee, S. (2011) Exact Relation for Correlation Functions in Compressible Isothermal Turbulence. Phys. Rev. Lett., 107(13), 134501.

[43] Garnier, E., Adams, N. \& Sagaut, P. (2009) Large Eddy Simulation for Incompressible Flows: An Introduction. Scientific Computation, Springer Netherlands.

[44] Germano, M. (1992) Turbulence: the filtering approach. J. Fluid Mech., 238.

[45] Geurts, B. J. (1997) Inverse modeling for large-eddy simulation. Phys. Fluids, $\mathbf{9}(12), 3585$.

[46] Glendinning, P. (1994) Stability, Instability and Chaos An Introduction to the Theory of Nonlinear Differential Equations. CUP.

[47] Goldstein, M. L. (2005) Turbulence on a small scale. Nature, 436(August), $1-2$.

[48] Grete, P., Vlaykov, D. G., Schmidt, W., Schleicher, D. R. G. \& FedERrATH, C. (2015) Nonlinear closures for scale separation in supersonic magnetohydrodynamic turbulence. New Journal of Physics, 17(2), 023070.

[49] Gunn, J. \& Peterson, B. (1965) On the Density of Neutral Hydrogen in Intergalactic Space. Astrophys. J., 142, 1633.

[50] Higashimori, K., Yokoi, N. \& Hoshino, M. (2013) Explosive turbulent magnetic reconnection. Phys. Rev. Lett., 110(25), 1-5.

[51] Iapichino, L., Maier, A., Schmidt, W. \& Niemeyer, J. C. (2010) Turbulence modeling and the physics of the intra-cluster medium. In AIP Conf. Proc., vol. 1241.

[52] Kolmogorov, A. N. (1962) A refinement of previous hypotheses concerning the local structure of turbulence in a viscous incompressible fluid at high Reynolds number. Journal of Fluid Mechanics, 13, 82-85. 
[53] Latif, M. A., Schleicher, D. R. G., Schmidt, W. \& Niemeyer, J. (2013) The small-scale dynamo and the amplification of magnetic fields in massive primordial haloes. Mon. Not. R. Astron. Soc., 432(1), 668-678.

[54] Leonard, A. (1974) Energy Cascade in Large-Eddy Simulations of Turbulent Fluid Flows. Advances in Geophysics, 18, A237.

[55] Liu, S., Meneveau, C. \& Katz, J. (1994) On the properties of similarity subgrid-scale models as deduced from measurements in a turbulent jet. J. Fluid Mech., 275(-1), 83.

[56] Love, M. D. (1980) Subgrid modelling studies with Burgers' equation. Journal of Fluid Mechanics, 100, 87-110.

[57] Madsen, K., Nielsen, H. B. \& Tingleff, O. (2004) Methods for non-linear least squares problems. "Informatics and Mathematical Modelling, Technical University of Denmark, $\{$ DTU $\} "$.

[58] Mangeney, A., Grappin, R. \& Velli, M. (1991) Magnetohydrodynamic Turbulence in the Solar Wind. In Advances in Solar System Magnetohydrodynamics, edited by E. R. Priest \& A. W. Hood.

[59] Miki, K. \& Menon, S. (2008) Localized dynamic subgrid closure for simulation of magnetohydrodynamic turbulence. Phys. Plasmas, 15(7), 072306.

[60] Moffatt, H. \& Tsinober, A. (1992) Helicity in laminar and turbulent flow. Annu. Rev. Fluid Mech., 24, 281-312.

[61] Müller, W.-C. \& CARAti, D. (2002) Dynamic gradient-diffusion subgrid models for incompressible magnetohydrodynamic turbulence. Phys. Plasmas, 9(3), 824 .

[62] MÜller, W.-C. \& CARATi, D. (2002) Large-eddy simulation of magnetohydrodynamic turbulence. Comput. Phys. Commun., 147, 544-547.

[63] Müller, W.-C., Malapaka, S. K. \& Busse, A. (2012) Inverse cascade of magnetic helicity in magnetohydrodynamic turbulence. Phys. Rev. E - Stat. Nonlinear, Soft Matter Phys., 85(1), 3-7.

[64] Ooi, A., Marting, J., Soria, J. \& Chong, M. S. (1999) A study of the evolution and characteristics of the invariants of the velocity-gradient tensor in isotropic turbulence. J. Fluid Mech., 381, S0022112098003681.

[65] Piomelli, U., Moin, P. \& Ferziger, J. H. (1988) Model consistency in large eddy simulation of turbulent channel flows. Phys. Fluids, 31(7), 1884.

[66] Pope, S. B. (2000) Turbulent Flows. Cambridge University Press, cambridge Books Online. 
[67] Pouquet, A., Frisch, U. \& LÉorat, J. (1976) Strong MHD helical turbulence and the nonlinear dynamo effect. Journal of Fluid Mechanics, 77, 321-354.

[68] PrandTL, L. (1925) Bericht über Untersuchungen zur ausgebildeten Turbulenz. Z. Angew. Math, Meth., 5, 136-139.

[69] PrandtL, L. (1945) Über ein neues Formelsystem für die ausgebildete Turbulenz. Nachr. Akad. Wiss. Göttingen Math-Phys. K1, 6-9, from Pope book.

[70] PRIEST, E. (2014) Magnetohydrodynamics of the Sun. CUP.

[71] Pruett, C. D., Sochacki, J. S. \& Adams, N. A. (2001) On Taylor-series expansions of residual stress. Phys. Fluids, 13(9), 2578.

[72] RÄDleR, K. H. (2014) Mean-field dynamos: The old concept and some recent developments. Astron. Nachrichten, 335(5), 459-469.

[73] Ragab, S. A. \& Sheen, S. (1992) The nonlinear development of supersonic instability waves in a mixing layer. Phys. Fluids A Fluid Dyn., 4(3), 553.

[74] Sagaut, P. (2006) Large Eddy Simulation for Incompressible Flows: An Introduction. Scientific Computation, Springer.

[75] Schekochihin, A. A., Iskakov, A. B., Cowley, S. C., McWilliams, J. C., Proctor, M. R. E. \& Yousef, T. A. (2007) Fluctuation dynamo and turbulent induction at low magnetic Prandtl numbers. New J. Phys., 9(8), 300-300.

[76] Schmidt, W. (2014) Large Eddy Simulations in Astrophysics. arXiv Prepr., 64.

[77] Schmidt, W. \& Federrath, C. (2011) A fluid-dynamical subgrid scale model for highly compressible astrophysical turbulence. Astron. Astrophys., 528, A106.

[78] Schmidt, W., Federrath, C., Hupp, M., Kern, S. \& Niemeyer, J. C. (2009) Numerical simulations of compressively driven interstellar turbulence. Astron. Astrophys., 494(1), 127-145.

[79] Schmidt, W., Niemeyer, J. C. \& Hillebrandt, W. (2006) A localised subgrid scale model for fluid dynamical simulations in astrophysics. Astron. Astrophys., 450(1), 265-281.

[80] Schmidt, W., Niemeyer, J. C., Hillebrandt, W. \& Roepke, F. K. (2006) A localised subgrid scale model for fluid dynamical simulations in astrophysics II: Application to type Ia supernovae. arXiv Prepr. astro-ph/, 3618(3618), 13.

[81] Smagorinsky, J. (1963) General Circulation Experiments with the Primitive Equations. Mon. Weather Rev., 91, 99.

[82] Soria, J., Sondergaard, R., Cantwell, B. J., Chong, M. S. \& Perry, A. E. (1994) A study of the fine-scale motions of incompressible time-developing mixing layers. Phys. Fluids, 6(2), 871. 
[83] Speziale, C. G., Erlebacher, G., Zang, T. A. \& Hussaini, M. Y. (1988) The subgrid-scale modeling of compressible turbulence. Phys. Fluids, 31(4), 940.

[84] Spruit, H. C. (2013) Essential Magnetohydrodynamics for Astrophysics. ArXiv e-prints:1301.5572.

[85] Stolz, S. \& AdAms, N. A. (1999) An approximate deconvolution procedure for large-eddy simulation. Phys. Fluids, 11(7), 1699.

[86] Stolz, S. \& Adams, N. A. (2003) Large-eddy simulation of high-Reynoldsnumber supersonic boundary layers using the approximate deconvolution model and a rescaling and recycling technique. Phys. Fluids, 15(8), 2398.

[87] Stolz, S., Adams, N. A. \& Kleiser, L. (2001) An approximate deconvolution model for large-eddy simulation with application to incompressible wall-bounded flows. Phys. Fluids, 13(4), 997.

[88] Stolz, S., Adams, N. A. \& Kleiser, L. (2001) The approximate deconvolution model for large-eddy simulations of compressible flows and its application to shock-turbulent-boundary-layer interaction. Phys. Fluids, 13(10), 2985.

[89] Stolz, S., Schlatter, P. \& Kleiser, L. (2005) High-pass filtered eddyviscosity models for large-eddy simulations of transitional and turbulent flow. Phys. Fluids, 17(6), 065103.

[90] Stolz, S., Schlatter, P. \& Kleiser, L. (2007) Large-Eddy Simulations of Subharmonic Transition in a Supersonic Boundary Layer. AIAA J., 45(5), 10191027.

[91] Subramanian, K., Shukurov, A. \& Haugen, N. E. L. (2006) Evolving turbulence and magnetic fields in galaxy clusters. Mon. Not. R. Astron. Soc., 366(4), 1437-1454.

[92] The Enzo Collaboration, Bryan, G. L., Norman, M. L., O’Shea, B. W., Abel, T., Wise, J. H., Turk, M. J., Reynolds, D. R. et Al. (2013) Enzo: An Adaptive Mesh Refinement Code for Astrophysics. wwwpublic.slac.stanford.edu, $\mathbf{2 1 1}(2), 60$.

[93] Theobald, M. L., Fox, P. A. \& Sofia, S. (1994) A subgrid-scale resistivity for magnetohydrodynamics. Phys. Plasmas, 1(9), 3016.

[94] Usmanov, A. V., Matthaeus, W. H., Breech, B. A. \& Goldstein, M. L. (2011) Solar wing modeling with turbulence transport and heating. Astrophys. J., 727(2), 84 .

[95] van Cittert, P. H. (1931) Zum Einflußder Spaltbreite auf die Intensitätsverteilung in Spektrallinien. II. Zeitschrift für Phys., 69(5-6), 298-308. 
[96] van der Bos, F., TaO, B., Meneveau, C. \& Katz, J. (2002) Effects of small-scale turbulent motions on the filtered velocity gradient tensor as deduced from holographic particle image velocimetry measurements. Phys. Fluids, 14(7), 2456 .

[97] VReman, B. (1995) Direct and Large-eddy Simulation of the Compressible Turbulent Mixing Layer. Ph.D. thesis, Twente University.

[98] Vreman, B., Geurts, B. \& Kuerten, H. (1996) Large-eddy simulation of the temporal mixing layer using the Clark model. Theor. Comput. Fluid Dyn., 8(4), 309-324.

[99] Vreman, B., Geurts, B. J. \& Kuerten, H. (1994) Realizability conditions for the turbulent stress tensor in large-eddy simulation. J. Fluid Mech., 278, $351-362$.

[100] Waagan, K., Federrath, C. \& Klingenberg, C. (2011) A robust numerical scheme for highly compressible magnetohydrodynamics: Nonlinear stability, implementation and tests. J. Comput. Phys., 230(9), 3331-3351.

[101] Wong, V. C. \& Lilly, D. K. (1994) A comparison of two dynamic subgrid closure methods for turbulent thermal convection. Phys. Fluids, 6(2), 1016.

[102] Woodward, P. R. \& Porter, D. H. (2006) Towards an Improved Numerical Treatment of Compressible Turbulence in Astrophysical Flows. Num. Mod. of Space Plasma Flows, 359, 97-110.

[103] Yeo, W. K. (1987) A Generalized High Pass/Low Pass Averaging Procedure for Deriving and Solving Turbulent Flow Equations. Ph.D. thesis, Ohio State University.

[104] YEO, W. K. (1993) Conjunctive filtering procedures in surface water flow and transport. In Large Eddy Simulation of Complex Engineering and Geophysical Flows, edited by B. Galperin \& S. A. Orszag, CUP, pp. 513-557.

[105] Yokoi, N. (2011) Modeling the turbulent cross-helicity evolution: production, dissipation, and transport rates. J. Turbul., 12(27), N27.

[106] Yokoi, N. (2013) Cross helicity and related dynamo. Geophys. Astrophys. Fluid Dyn., 107(1-2), 114-184.

[107] Yokoi, N. \& Hamba, F. (2007) An application of the turbulent magnetohydrodynamic residual-energy equation model to the solar wind. Phys. Plasmas, 14(11), 112904.

[108] Yokoi, N., Higashimori, K. \& Hoshino, M. (2013) Transport enhancement and suppression in turbulent magnetic reconnection: A self-consistent turbulence model. Phys. Plasmas, 20(12), 122310. 
[109] YoshizawA, A. (1990) Selfconsistent turbulent dynamo modeling of reversed field pinches and planetary magnetic fields. Phys. Fluids B Plasma Phys., 2(7), 1589 .

[110] Yoshizawa, A. (1991) Subgrid-scale modeling of compressible turbulent flows. Phys. Fluids A Fluid Dyn., 3(4), 714.

[111] YoshizawA, A. (1991) Subgrid-Scale modelling of Magentohydrodynamic Turbulence. J. Phys. Soc. Japan, 60(1), 9-12.

[112] Yoshizawa, A. (1995) Turbulent MHD Dynamo: Modeling of the Turbulent Residual-Helicity Equation.

[113] Yoshizawa, A. \& Iтон, S. (2001) Turbulence theories and modelling of fluids and plasmas. Plasma Phys., 1.

[114] Yoshizawa, A. \& Itoh, S. (2004) Dynamos and MHD theory of turbulence suppression. Plasma Phys., 46(3), R25-R94.

[115] Zelenyi, L. M. \& Milovanov, A. V. (2004) Fractal topology and strange kinetics: from percolation theory to problems in cosmic electrodynamics. Uspekhi Fiz. Nauk, 174(8), 809.

[116] Zhou, Y., Matthaeus, W. \& Dmitruk, P. (2004) Colloquium: Magnetohydrodynamic turbulence and time scales in astrophysical and space plasmas. Rev. Mod. Phys., 76(4), 1015-1035.

[117] Zhou, Y. \& VAhala, G. (1991) Aspects of subgrid modelling and large-eddy simulation of magnetohydrodynamic turbulence. J. Plasma Phys., 45(11141), 239-249. 



\section{Acknowledgements}

Foremost, I would like thank my supervisor Wolfram Schmidt for giving me the opportunity to pursues this research in the first place. Thank you for your flexibility, patience, guidance, and expertise, and for allowing me the freedom to pursue this topic in the directions it led me despite the many dead ends. This work would not have been possible without the endless debates and unrelenting support of Philipp Grete, who showed me the door out of k-space. I am grateful to Dominik Schleicher for many insightful discussions. I thank Christoph Federrath for allowing me access to his turbulence data. Special thanks to all the committee members for dealing with all the last minute changes.

This research was made possible through the financial support of the Deutsche Forschungsgemeinschaft (DFG) under grant SFB 963/1, project A15. The data for this work was in part produced and analysed at the Leibniz Rechenzentrum and the Gauss Centre for Supercomputing (grant pr32lo), the Partnership for Advanced Computing in Europe (PRACE grant pr98mu). The software used in this work was in part developed by the DOE-supported Flash Center for Computational Science at the University of Chicago. Last but not least, I am grateful to my family and my partner Laura Volpes for their tireless motivational and creative support and their understanding. 